\title{
Catalytic asymmetric aza-Michael addition of fumaric monoacids with multifunctional thiourea/boronic acids
}

\section{$\operatorname{AUTHOR}(S)$ :}

Michigami, Kenichi; Murakami, Hiroki; Nakamura, Takeru; Hayama, Noboru; Takemoto, Yoshiji

\section{CITATION:}

Michigami, Kenichi ... [et al]. Catalytic asymmetric aza-Michael addition of fumaric monoacids with multifunctional thiourea/boronic acids. Organic and Biomolecular Chemistry 2019, 17(9): 2331-2335

\section{ISSUE DATE:}

2019-03-07

URL:

http://hdl.handle.net/2433/242994

\section{RIGHT:}

This is the accepted manuscript of the article, which has been published in final form at

https://doi.org/10.1039/C90B00045C.; The full-text file will be made open to the public on 29 January 2020 in

accordance with publisher's 'Terms and Conditions for Self-Archiving'.; This is not the published version. Please cite only the published version.; この論文は出版社版でありません。引用の際には出版社版をご確認ご利用ください。 


\section{Journal Name}

\section{COMMUNICATION}

\section{Catalytic asymmetric aza-Michael addition of fumaric monoacids with multifunctional thiourea/boronic acids}

Received 00th January 20xx, Accepted 00th January 20xx

DOI: $10.1039 / \times 0 \times x 00000 x$
Kenichi Michigami, Hiroki Murakami, Takeru Nakamura, Noboru Hayama, and Yoshiji Takemoto*

www.rsc.org/

The first chemical enantioselective synthesis of $\mathbf{N}$ hydroxyaspartic acid derivatives using chiral multifunctional thiourea/boronic acid organocatalysts was developed. A series of fumaric monoacids underwent an intermolecular asymmetric aza-Michael addition of $\boldsymbol{O}$-alkyl hydroxylamines in excellent regioselectivity. The addition of another carboxylic acid raised the enantiomeric enrichment up to $97 \%$ ee. $O$-Deprotection of the aza-Michael adduct provided an aspartate-derived hydroxylamine fragment applicable for KAHA ( $\alpha$-keto acidhydroxylamine) ligation.

Chemoselective peptide conjunction by enzymes is a ubiquitous process in nature, producing only recyclable phosphates as byproducts. ${ }^{1}$ Meanwhile, a general chemical approach for the amide $\mathrm{C}-\mathrm{N}$ bond formation is based on stoichiometric activation of carboxylic acids by coupling reagents. ${ }^{2}$ Though this method is highly reliable, reagent-derived non-recyclable side products are inevitably generated. In addition, protection of nucleophilic functional groups is required due to incompatibility with condensation reagents. The development of environmentally benign alternatives is thus highly demanded. ${ }^{3}$ Among large numbers of strategies reported to circumvent these issues, elegant examples have been disclosed in recent years by Bode and co-workers. The protocol of simply mixing $\alpha$-keto acids and hydroxylamines in aqueous solvent under mild conditions afford amides concomitant with the release of carbon dioxide $\left(\mathrm{CO}_{2}\right)$ and water. ${ }^{4}$ This clean amide synthesis, KAHA ligation, has manifested significant advantages in polypeptide synthesis because the connection of peptide segments proceeds without protection of side chains and the loss of enantiopurity. ${ }^{4 a}$ However, low accessibility of both $\alpha$-keto acids and hydroxylamines underlies a major problem that affects the practical convenience. In marked contrast to $\alpha$-amino acid-derived $\alpha$-keto acids, ${ }^{5}$ synthetic

Graduate School of Pharmaceutical Sciences, Kyoto University, Yoshida, Sakyo-ku, Kyoto 606-8501, Japan. E-mail: takemoto@pharm.kyoto-u.ac.jp

†Electronic Supplementary Information (ESI) available: [experimental procedures, characterization data, copies of ${ }^{1} \mathrm{H}$ and ${ }^{13} \mathrm{C} N M R$ spectra, and copies of HPLC analyses (PDF)]. See DOI: 10.1039/x0xx00000x research for hydroxylamine counterparts is still less exploited: 6 only a few simple derivatives including cyclic analogues of homoserine, serine, and aspartic acid semialdehyde (Asa) have been synthesised and applied to KAHA peptide ligation (Scheme 1A). ${ }^{4 b, 6 d, 7}$ Since these nucleophiles require long-step routes for preparation, a straightforward and enantioselective synthesis of such hydroxylamines represents a major challenge for KAHA strategy in view of general peptide synthesis.

(A) Precedented Hydroxylamine Fragments for KAHA Peptide Ligation

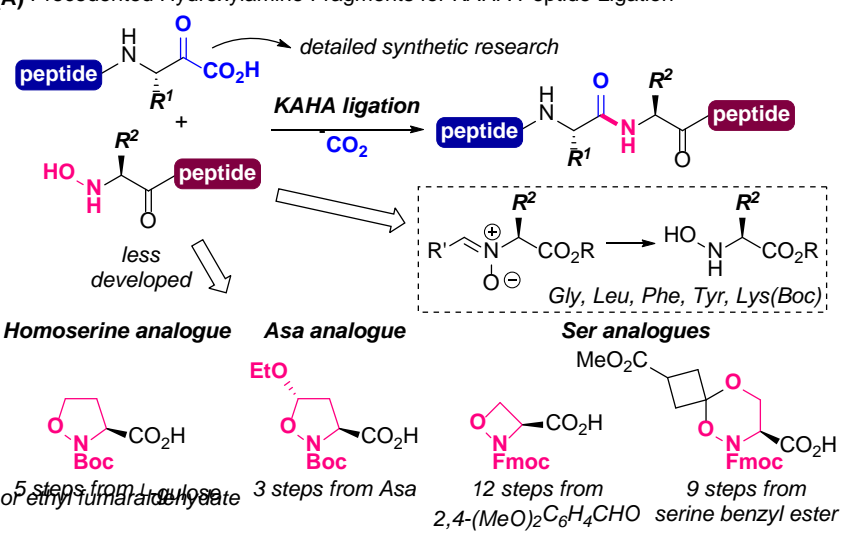

(B) Catalytic Asymmetric Aza-Michael Addition of ${ }^{\alpha}{ } \beta$-Unsaturated Carboxylic Acids

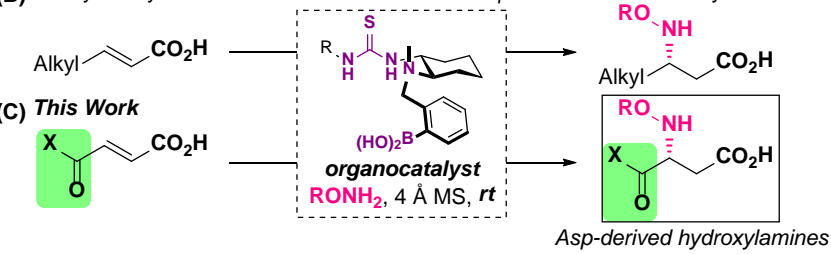

Scheme 1. Strategy for the Synthesis of $\alpha$-Amino Acid-Derived Hydroxylamines for KAHA Peptide Ligation

We have recently focused on a direct catalytic asymmetric azaMichael addition of $\mathrm{BnONH}_{2}$ to $\alpha, \beta$-unsaturated carboxylic acids using organocatalysts consisting of arylboronic acid and chiral trans-1,2-cyclohexanediamine-based aminothiourea (Scheme 1B). ${ }^{8-10}$ Notably, our multifunctional catalysts only promoted 1,4addition: The 1,2-adducts, $\mathrm{N}$-benzyloxyamides, which are usually formed in organoboron-catalysed dehydrative amidation, ${ }^{11}$ were 
not observed. Encouraged by the efficient protocols free from "pre-activation" and "protection" of carboxylic acids,, we targeted $\mathrm{N}$-hydroxyaspartic acid derivatives to demonstrate synthetic versatility of our catalytic systems. Although biocatalytic synthesis of enantiopure $\mathrm{N}$-hydroxy and $\mathrm{N}$ alkoxyaspartates have been reported, ${ }^{13}$ chemical methods are still under developed, and to the best of our knowledge, no example has emerged for catalytic asymmetric variant. ${ }^{14}$ Herein, we report the asymmetric synthesis of $N$-hydroxyaspartic acid derivatives catalysed by multifunctional thiourea/boronic acids, which would offer a new, facile access to aspartate-derived hydroxylamine fragments suitable for KAHA peptide ligation.

First, initial investigations focused on catalytic asymmetric azaMichael addition using mono-tert-butyl fumarate (1a) and $O$ benzylhydroxylamine (2a) as a nucleophile. In line with our previous studies, a chiral integrated thiourea/boronic acid catalyst A was employed in the presence of $4 \AA$ molecular sieves (4 A MS) at room temperature. ${ }^{8 c}$ Though no reaction took place in DMF and MeCN (Table 1, entries 1 and 2), the reaction in less polar solvents, $\mathrm{Et}_{2} \mathrm{O}$ and $\mathrm{CH}_{2} \mathrm{Cl}_{2}$, furnished the corresponding $\mathrm{N}$ benzyloxyaspartic acid diester 3 aa after treatment with $\mathrm{TMSCHN}_{2}$ (entries 3 and 4). When the reaction was conducted in toluene, both the yield and ee drastically increased (entry 5). $\mathrm{CCl}_{4}$ was found to be the optimal solvent, providing $3 a a$ in $80 \%$ yield and $88 \%$ ee (entry 6 ). Next, thiourea moiety of the catalyst was deviated. Loss of both yields and ees was observed with electronwithdrawing thioureas B and C (entries 7 and 8). The ortho-Me substituted catalyst D gave slightly higher ee, but the yield dropped to $59 \%$. N-Methyl thiourea catalyst $\mathrm{E}$ also promoted the reaction, albeit in moderate yield and ee (entry 10). Therefore, we continued the following experiments with catalyst $\mathbf{A}$. Afterwards, a range of carboxylic acid was employed as additives, since our previous works suggested three molecules of carboxylic acid were involved in catalytic cycle: Two of them bind to the catalyst and the other one works as a proton shuttle (vide infra). ${ }^{8 \mathrm{c}}$ No reaction occurred with 1 equivalent of $\mathrm{HCO}_{2} \mathrm{H}$, and $\mathrm{AcOH}$ or ${ }^{t} \mathrm{BuCO}_{2} \mathrm{H}$ did not exhibit beneficial effects. Meanwhile, both the yield and ee improved with $\mathrm{PhCO}_{2} \mathrm{H}$, affording 3aa in $88 \%$ yield and $93 \%$ ee (entry 14). In sharp contrast, a highly acidic $p$-TsOH inhibited the reaction completely, probably due to the protonation of the nucleophile and/or the catalyst (entry 15). Control experiments revealed that both the catalyst and $4 \AA$ MS were essential for the reaction (entries 16 and 17). All reactions proceeded at $\beta$ position of the carboxylic acid exclusively, which indicates that the carboxylic acid was predominantly activated in situ by boronic acid over the alkyl ester moiety. ${ }^{15}$

Table 1. Optimisation of the Reaction Conditions.

\begin{tabular}{|c|c|c|c|c|c|}
\hline \multirow[b]{2}{*}{ entry } & \multirow{2}{*}{$\begin{array}{c}\mathrm{IO}_{2} \mathrm{C} \\
\text { catalyst }\end{array}$} & \multicolumn{2}{|c|}{$\begin{array}{c}\text { catalyst (10 mol\%) } \\
\stackrel{\mathrm{BnONH}_{2} 2 \mathrm{2a}(1.1 \text { equiv) }}{\longrightarrow} \\
\begin{array}{c}\text { additive (1 equiv) } \\
4 \AA \mathrm{MS}, \text { solvent, rt, } 24 \mathrm{~h} ; \\
\text { then } \mathrm{TMSCHN}_{2}\end{array}\end{array}$} & $\mathrm{BuO}_{2} \mathrm{C} \overbrace{3 \mathbf{B a}}^{\mathrm{BnO}_{-\mathrm{NH}}}$ & ${ }_{2} \mathrm{Me}$ \\
\hline & & additive & solvent & 3aa (\%) ${ }^{a}$ & ee $(\%)^{b}$ \\
\hline 1 & A & - & DMF & 0 & - \\
\hline 2 & A & - & $\mathrm{MeCN}$ & 0 & - \\
\hline 3 & A & - & $\mathrm{Et}_{2} \mathrm{O}$ & 22 & 28 \\
\hline 4 & A & - & $\mathrm{CH}_{2} \mathrm{Cl}_{2}$ & 10 & 47 \\
\hline
\end{tabular}

\begin{tabular}{cccccc}
5 & A & - & toluene & 60 & 69 \\
6 & A & - & $\mathrm{CCl}_{4}$ & 80 & 88 \\
7 & $\mathbf{B}$ & - & $\mathrm{CCl}_{4}$ & 66 & 80 \\
8 & $\mathbf{C}$ & - & $\mathrm{CCl}_{4}$ & 72 & 76 \\
9 & $\mathbf{D}$ & - & $\mathrm{CCl}_{4}$ & 59 & 90 \\
10 & $\mathbf{E}$ & - & $\mathrm{CCl}_{4}$ & 62 & 80 \\
11 & $\mathbf{A}$ & $\mathrm{HCO}_{2} \mathrm{H}$ & $\mathrm{CCl}_{4}$ & 0 & - \\
12 & $\mathbf{A}$ & $\mathrm{MeCO}_{2} \mathrm{H}$ & $\mathrm{CCl}_{4}$ & 57 & 91 \\
13 & $\mathbf{A}$ & ${ }^{\mathrm{BuCO}_{2} \mathrm{H}}$ & $\mathrm{CCl}_{4}$ & 77 & 87 \\
14 & $\mathbf{A}$ & $\mathrm{PhCO}_{2} \mathrm{H}$ & $\mathrm{CCl}_{4}$ & 88 & 93 \\
15 & A & $p-\mathrm{TsOH}_{4} \mathrm{H}_{2} \mathrm{O}$ & $\mathrm{CCl}_{4}$ & 0 & - \\
16 & none & - & $\mathrm{CCl}_{4}$ & 0 & - \\
$17 c$ & A & - & $\mathrm{CCl}_{4}$ & 0 & - \\
\hline
\end{tabular}

Reaction conditions: 1 a $(0.10 \mathrm{mmol}), 2 \mathrm{a}(0.11 \mathrm{mmol}, 1.1$ equiv), catalyst $(0.010 \mathrm{mmol}, 10 \mathrm{~mol} \%)$, additive $(0.10 \mathrm{mmol}, 1.0$ equiv), $4 \AA$ MS (50 mg), solvent $(1.0 \mathrm{~mL}, 0.1 \mathrm{M}), \mathrm{rt}, 24 \mathrm{~h}$. ${ }^{a}$ Isolated yields are shown. ${ }^{b}$ Ees were estimated by chiral HPLC analysis. ${ }^{c}$ The reaction was performed without $4 \AA \mathrm{MS}$.

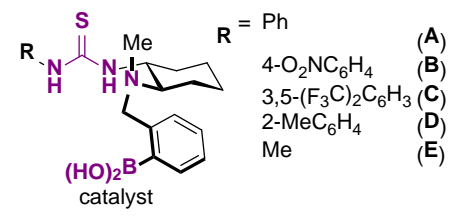

With the optimal conditions in hand, we next explored the substrate scope of the aza-Michael addition of fumaric acid monoesters with $\mathbf{2 a}$ in the presence of catalyst $\mathbf{A}$. (Figure 1 ). Benzyl ester $\mathbf{1 b}$ and ethyl ester $\mathbf{1} \mathbf{c}$ were converted into the corresponding aspartates $\mathbf{3 b a}$ and $\mathbf{3} \mathbf{c a}$, respectively in moderate yields with high ees. Remarkably, in addition to fumaric acid monoesters, phenylalanine-derived fumaric acid monoamides $\mathbf{1 d}$ and 1e underwent the aza-Michael addition. By employing catalyst $\mathbf{A}$ with $(R, R)$ configuration, D-Phe-D-Asp derivative 3ea was furnished in higher diastereoselectivity than that of L-Phe-DAsp derivative $\mathbf{3 d a}$. The catalyst was switched to $(S, S)$-A (ent-A) for the reaction of $N$-fumaryl-L-amino esters $1 \mathbf{1 f}$ and $\mathbf{1 g}$, producing Gly-L-Asp and L-Ser-L-Asp derivatives $\mathbf{3 f a}$ and 3ga, respectively. Even though the stereoselectivity is moderate, these results suggest the feasibility of the synthesis of $N$-hydroxyaspartatederived peptides through this protocol. 


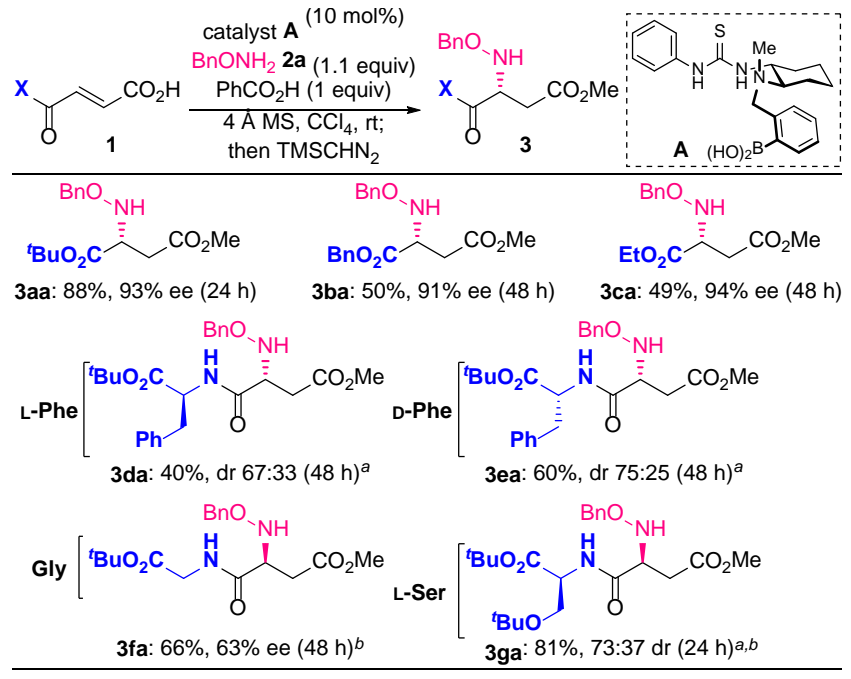

Figure 1. Substrate scope of fumaric monoacids $(0.40 \mathrm{mmol})$. Isolated yields are shown. Ees were estimated by chiral HPLC analysis. ${ }^{a} \mathrm{Dr}$ was determined by ${ }^{1} \mathrm{H}$ NMR analysis. ${ }^{\text {bent-A }}$ was used as a catalyst.

The efficiency and high stereoselectivity of the aza-Michael addition of $\mathrm{BnONH}_{2}$ led us to the examination of various $O$ substituted hydroxylamines. To evaluate $O$-substituent effect of nucleophiles $\mathbf{2 a - 2 e}$ on ee, the aza-Michael addition of $\mathbf{1 a}$ was examined under the optimal conditions (Figure 2). The use of electron-rich hydroxylamine $\mathbf{2} \mathbf{b}$ resulted in lower yield and enantioselectivity, whereas electron-deficient $p-\mathrm{CF}_{3}$ substituted nucleophile 2c gave 3 ac in $96 \%$ ee. Besides, acetal-protected nucleophiles such as BOMONH${ }_{2}(\mathbf{2 d})$ and $\mathrm{SEMONH}_{2}(\mathbf{2 e})$ were also competent nucleophiles, affording the corresponding adducts 3ad and 3ae in good yields and enantioselecitivies.

$$
\text { 2a: } 88 \%, 93 \% \text { ee }
$$

Figure 2. Screening of Nucleophiles $(0.20-0.40 \mathrm{mmol})$. Isolated yields are shown. Ees were estimated by chiral HPLC analysis. ${ }^{a}$ Ee was determined after $N$-benzoylation.

Based on the experimental results, including perfect regioselectivity and mechanistic studies performed previously, ${ }^{8 c}$ a proposed transition state of the addition of hydroxylamine is depicted in Figure 3. Since no reaction occurred in the absence of $4 \AA$ AS (Table 1, entry 17), removal of water is indispensable for this reaction. In a plausible intermediate containing diacyloxyboronate, the hydrogen-bond interaction between the thiourea NH protons and one of the carboxy group facilitates conjugate addition of hydroxylamine. The high enantioselectivity would be attributed to an additional interaction between the nucleophile $\mathrm{NH}$ proton and another carboxy ligand, accelerating Re-face attack of hydroxylamine. The nucleophilic addition is accompanied with intermolecular proton transfer, which is promoted by the third molecule of carboxylic acid.

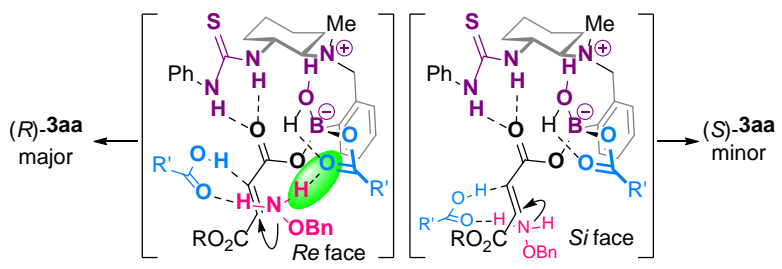

Figure 3. Proposed Transition State of Nucleophilic Addition.

A quick access to various chiral $\mathrm{N}$-alkoxyaspartate derivatives prompted us to examine $O$-deprotection of the Michael adducts for the application to KAHA ligation. Various deprotection conditions tested revealed that compound $\mathbf{3 b e}$, obtained from $\mathbf{1 b}$ and $\mathbf{2 e}$, was the substrate of choice. First, treatment of $\mathbf{3 b e}$ with TFA and subsequent careful quenching with water gave an $\mathrm{OH}$ free hydroxylamine $\mathbf{4 b}$ in $42 \%$ yield. ${ }^{15,16}$ The following KAHA ligation using $\alpha$-keto acid 5 proceeded smoothly and the corresponding amide $\mathbf{6} \mathbf{b}$ was obtained without significant loss of enantiomeric excess. ${ }^{7 a}$ The same reaction with Fmoc-L-leucinederived $\alpha$-keto acid $\mathbf{7}^{17}$ also afforded dipeptide $\mathbf{8 b}$ in moderate yield without epimerisation.

Scheme 3. $O$-Deprotection and KAHA Ligation of AsparatateDerived Hydroxylamine Derivatives

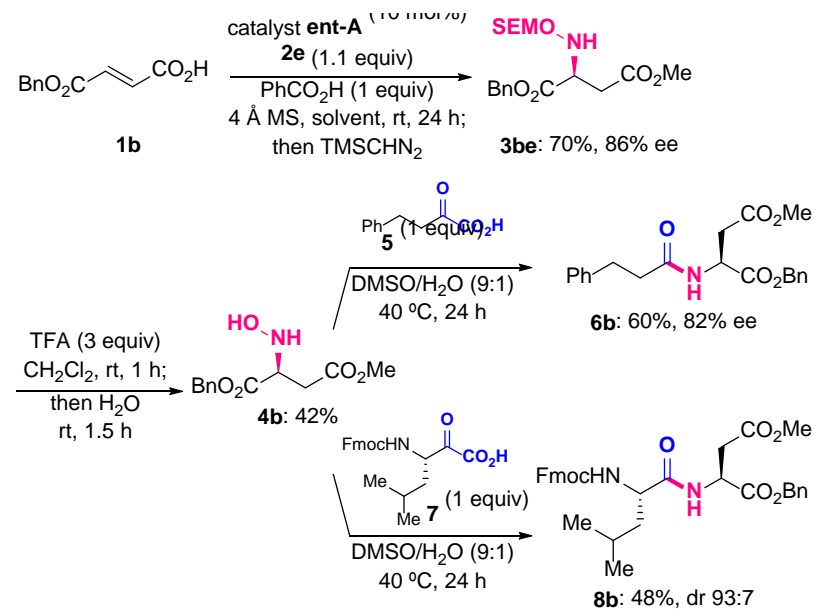

In summary, we have developed a direct aza-Michael addition of hydroxylamine derivatives to various fumaric monoacids catalysed by multifunctional thiourea/boronic acids. The process enables the first catalytic asymmetric chemical synthesis of $\mathrm{N}$-hydroxyaspartic acid derivatives with perfect regioselectivity and high enantioselectivity. Deprotection of the SEMONH $\mathrm{S}_{2}$-adduct provides a new aspartate-derived hydroxylamine fragment for KAHA peptide ligation. Further research into the synthetic application is actively ongoing, and the results will be reported in due course.

\section{Conflicts of interest}

There are no conflicts to declare. 


\section{Acknowledgement}

This paper is dedicated to the memory of our talented colleague, $\mathrm{Mr}$. Takeru Nakamura, who passed away on 25 October, 2017. This work was financially supported by JSPS KAKENHI, Grant No. JP16H06384.

\section{Notes and references}

1 Recent reviews on enzymatic amide $\mathrm{C}-\mathrm{N}$ bond formation: (a) K. Yazawa, K. Numata, Molecules, 2014, 19, 13755; (b) A Goswami, S. G. V. Lanen, Mol. BioSyst., 2015, 11, 338; (c) J. Pitzer, K. Steiner, J. Biotechnol., 2016, 235, 32; (d) M. Winn, J. K. Fyans, Y. Zhuo, J. Micklefield, Nat. Prod. Rep., 2016, 33, 317. (e) R. D. Süssmuth, A. Mainz, Angew. Chem., Int. Ed., 2017, 56, 3770.

2 Selected reviews on amide synthesis using coupling reagents: (a) C. A. G. N. Montalbetti, V. Falque, Tetrahedron, 2005, 61, 10827; (b) E. Valeur, M. Bradley, Chem. Soc. Rev., 2009, 38, 606; (c) A. El-Faham, F. Albericio, Chem. Rev., 2011, 111, 6557.

3 A recent review on nonclassical amidation: (a) C. L. Allen, J. M J. Williams, Chem. Soc. Rev., 2011, 40, 3405; (b) R. M. de Figueiredo, J.-S. Suppo, J.-M. Campagne, Chem. Rev., 2016, 116, 12029.

4 (a) J. W. Bode, R. M. Fox, K. D. Baucom, Angew. Chem., Int. Ed. 2006, 45, 1248; (b) A. K. Sanki, R. S. Talan, S. J. Sucheck, J. Org. Chem., 2009, 74, 1886; (c) I. Pusterla, J. W. Bode, Angew. Chem., Int. Ed., 2012, 51, 513.

5 (a) L. Ju, A. R. Lippert, J. W. Bode, J. Am. Chem. Soc., 2008, 130 4253; (b) L. Ju, J. W. Bode, Org. Biomol. Chem., 2009, 7, 2259; (c) T. J. Harmand, S. S. Kulkarni, J. W. Bode, Tetrahedron Lett., 2015, 56, 3477.

6 Selected examples of the synthesis of $\alpha$-amino acid-derived hydroxylamines. (a) G. Grundke, W. Keese, M. Rimper, Synthesis, 1987, 1115; (b) W. Oppolzer, O. Tamura, Tetrahedron Lett., 1990, 31, 991. (c) M. D. Wittman, R. L. Halcomb, S. J. Danishefsky, J. Org. Chem. 1990, 55, 1981; (d) S. I. Medina, J. Wu, J. W. Bode, Org. Biomol. Chem., 2010, 8, 3405; (e) C. D. McCune, S. J. Chan, M. L. Beio, W. Shen, W. J. Chung, L. M. Szczesniak, C. Chai, S. Q. Koh, P. T.-H. Wong, D. B. Berkowitz, ACS Cent. Sci., 2016, 2, 242.

7 Selected examples of the synthesis and applications of $\alpha$ amino acid-derived cyclic hydroxylamines: (a) V. R Pattabiraman, A. O. Ogunkoya, J. W. Bode, Angew. Chem., Int. Ed., 2012, 51, 5114; (b) C. E. Murar, F. Thuaud, J. W. Bode, J. Am. Chem. Soc., 2014, 136, 18140; (c) I. Pusterla, J. W. Bode, Nat. Chem., 2015, 7, 668; (d) C. E. Murar, T. J. Harmand, J. W. Bode, Bioorg. Med. Chem., 2017, 25, 4996; (e) T. J Harmand C. E. Murar, H. Takano, J. W. Bode, Org. Synth. 2018, 95, 142; (f) C. E. Murar, T. J. Harmand, H. Takano, J. W. Bode, Org. Synth. 2018, 95, 157. (g) S. Baldauf, J. W. Bode, Synthesis, 2019, DOI: $10.1055 / \mathrm{s}-0037-1611635$.

8 (a) T. Azuma, A. Murata, Y. Kobayashi, T. Inokuma, Y Takemoto, Org. Lett., 2014, 16, 4256; (b) N. Hayama, T. Azuma, Y. Kobayashi, Y. Takemoto, Chem. Pharm. Bull., 2016, 64, 704; (c) N. Hayama, R. Kuramoto, T. Földes, K. Nishibayashi, Y. Kobayashi, I. Pápai, Y. Takemoto, J. Am. Chem. Soc. 2018, 140 12216.

9 Selected examples of catalytic transformation of carboxylic acids without converting the acid moieties: (a) R. Giri, N. Maugel, J.-J. Li, D.-H. Wang, S. P. Breazzano, L. B. Saunders, J.Q. Yu, J. Am. Chem. Soc., 2007, 129, 3510; (b) R. M. Al-Zoubi, O. Marion, D. G. Hall, Angew. Chem., Int. Ed., 2008, 47, 2876; (c) H. Zheng, R. McDonald, D. G. Hall, Chem. Eur. J., 2010, 16 5454; (d) Y. Morita, T. Yamamoto, H. Nagai, Y. Shimizu, M. Kanai, J. Am. Chem. Soc., 2015, 137, 7075; (e) H. Nagai, Y. Morita, Y. Shimizu, M. Kanai, Org. Lett., 2016, 18, 2276; (f) M.
Simonetti, D. M. Cannas, A. Panigrahi, S. Kujawa, M. Kryjewski, P. Xie, I. Larrosa, Chem. Eur. J., 2017, 23, 549 and references cited therein.

10 Recent reviews on aza-Michael Addition: (a) D. Enders, C. Wang, J. X. Liebich, Chem. Eur. J., 2009, 15, 11058; (b) P. R. Krishna, A. Sreeshailam, R. Srinivas, Tetrahedron, 2009, 65, 9657; (c) Z. Amara, J. Caron, D. Joseph, Nat. Prod. Rep., 2013, 30, 1211; (d) J. Gmach, Ł. Joachimiak, K. M. Błażewska, Synthesis, 2016, 48, 2681.

11 Selected examples of organoboron-catalysed dehydrative amidation: (a) K. Ishihara, S. Ohara, H. Yamamoto, J. Org. Chem., 1996, 61, 4196; (b) K. Arnold, B. Davies, D. Hérault, A. Whiting, Angew. Chem., Int. Ed., 2008, 47, 2673; (c) P. Starkov, T. D. Sheppard, Org. Biomol. Chem., 2011, 9, 1320; (d) N. Gernigon, R. M. Al-Zoubi, D. G. Hall, J. Org. Chem., 2012, 77, 8386; (e) T. M. E. Dine, W. Erb, Y. Berhault, J. Rouden, J. Blanchet, J. Org. Chem., 2015, 80, 4532; (f) H. Noda, M. Furutachi, Y. Asada, M. Shibasaki, N. Kumagai, Nat. Chem., 2017, 9, 571. (g) K. Wang, Y. Lu, K. Ishihara, Chem. Commun., 2018, 54, 5410. (h) D. N. Sawant, D. B. Bagal, S. Ogawa, K. Selvam, S. Saito, Org. Lett. 2018, 20, 4397.

12 Selected examples of Michael addition of $\alpha, \beta$-unsaturated carboxylic acid derivatives: (a) Y. Hamashima, H. Somei, Y. Shimura, T. Tamura, M. Sodeoka, Org. Lett., 2004, 6, 1861; (b) N. Yamagiwa, H. Qin, S. Matsunaga, M. Shibasaki, J. Am. Chem. Soc., 2005, 127, 13419; (c) S. Kikuchi, H. Sato, S. Fukuzawa, Synlett, 2006, 1023; (d) M. P. Sibi, K. Itoh, J. Am. Chem. Soc., 2007, 129, 8064; (e) M. Weiß, S. Borchert, E. Rémond, S. Jugé, H. Gröger, Heteroatom. Chem., 2012, 23, 202; (f) Y. Kobayashi, Y. Taniguchi, N. Hayama, T. Inokuma, Y. Takemoto, Angew. Chem., Int. Ed., 2013, 52, 11114 and references cited therein.

13 (a) M. S. Gulzar, M. Akhtar, D. Gani, J. Chem. Soc., Chem. Commun., 1994, 1601; (b) B. Weiner, G. J. Poelarends, D. B. Janssen, B. L. Feringa, Chem. Eur. J., 2008, 14, 10094; (c) V. P. Veetil, H. Raj, M. de Villiers, P. G. Tepper, F. J. Dekker, W. J. Quax, G. J. Poelarends, Chem CatChem, 2013, 5, 1325.

14 (a) J. E. Baldwin, L. M. Harwood, M. J. Lombard, Tetrahedron, 1984, 40, 4363; (b) T. Kolasa, Can. J. Chem., 1985, 63, 2139; (c) R. W. Feenstra, E. H. M. Stokkingreef, R. J. F. Nivard, H. C. J. Ottenheijm, Tetrahedron, 1988, 44, 5583; (d) S. B. McNabb, M. Ueda, T. Naito, Org. Lett., 2004, 6, 1911; (e) N. Tibrewal, G. I. Elliott, Bioorg. Med. Chem. Lett., 2011, 21, 517.

15 For the details of optimisations, see the Supporting Information.

16 R. H. Schlessinger, M. A. Poss, S. Richardson, J. Am. Chem. Soc., 1986, 108, 3112.

17 (a) F. Thuaud, F. Rohrbacher, A. Zwicky, J. W. Bode, Helv. Chim. Acta, 2016, 99, 868. (b) T. Nanjo, N. Kato, Y. Takemoto, Org. Lett., 2018, 20, 5766. 


\section{Catalytic asymmetric aza-Michael addition of fumaric monoacids with multifunctional thiourea/boronic acids}

Kenichi Michigami, Hiroki Murakami, Takeru Nakamura, Noboru Hayama and Yoshiji Takemoto*

Graduate School of Pharmaceutical Sciences, Kyoto University, Yoshida Sakyo-ku, Kyoto 606-8501, Japan

takemoto@pharm.kyoto-u.ac.jp

Supporting Information 


\section{Table of Contents}
(A) Supplemental Data
(A-1) Optimisation Details: Tables S1-S6
S3
(A-2) Determination of Stereochemistry S5
(B) General $\quad \mathrm{S6}$
(C) Materials and Methods
(C-1) Preparation of Substrates $\quad$ S7
(C-2) Preparation of Nucleophiles $\quad$ S10
(C-3) General Procedure for Catalytic Aza-Michael Addition S12
(D) $O$-Deprotection and KAHA Ligation $\quad \mathrm{S} 18$
(E) References $\quad$ S20
(F) ${ }^{1} \mathrm{H}$ NMR and ${ }^{13} \mathrm{C}$ NMR Spectra $\quad \mathrm{S} 21$
$\begin{array}{ll}\text { (G) HPLC Traces } & \text { S87 }\end{array}$ 


\section{(A) Supplemental Data}

\section{(A-1) Optimisation Details: Tables S1-S8}

Several reaction parameters of the enantioselective aza-Michael addition were investigated. In each tables are described isolated yields.

Table S1. Investigation of Solvent Effect

\begin{tabular}{|c|c|c|c|c|c|c|c|}
\hline \multicolumn{2}{|c|}{$1 a$} & \multicolumn{2}{|c|}{$\begin{array}{c}\text { MS4A, solvent }(0.2 \mathrm{M}) \\
\text { rt, } 24 \mathrm{~h} ; \\
\text { then } \mathrm{TMSCHN}_{2}\end{array}$} & \multicolumn{2}{|c|}{$\mathrm{O}_{2} \overbrace{3 \mathbf{a a}}^{\mathrm{BnO}_{-\mathrm{NH}}}$} & & st $A$ \\
\hline entry & solvent & Заа (\%) & ee $(\%)$ & entry & solvent & Заа (\%) & еe (\%) \\
\hline 1 & DMF & 0 & - & 7 & hexane & 35 & 38 \\
\hline 2 & $\mathrm{MeCN}$ & 0 & - & 8 & $\mathrm{CCl}_{4}$ & 70 & 88 \\
\hline 3 & EtOAc & 0 & - & 9 & $\mathrm{C}_{2} \mathrm{Cl}_{4}$ & 70 & 81 \\
\hline 4 & $\mathrm{Et}_{2} \mathrm{O}$ & 22 & 28 & 10 & $4-\mathrm{F}_{3} \mathrm{CC}_{6} \mathrm{H}_{4} \mathrm{Cl}$ & 55 & 73 \\
\hline 5 & $\mathrm{CH}_{2} \mathrm{Cl}_{2}$ & 10 & 47 & 11 & $\mathrm{PhF}$ & 15 & 62 \\
\hline 6 & toluene & 60 & 69 & 12 & $\mathrm{PhCl}$ & 29 & 7 \\
\hline
\end{tabular}

Table S2. Deviation of Thioureas of Multifunctional Organoboron Catalysts

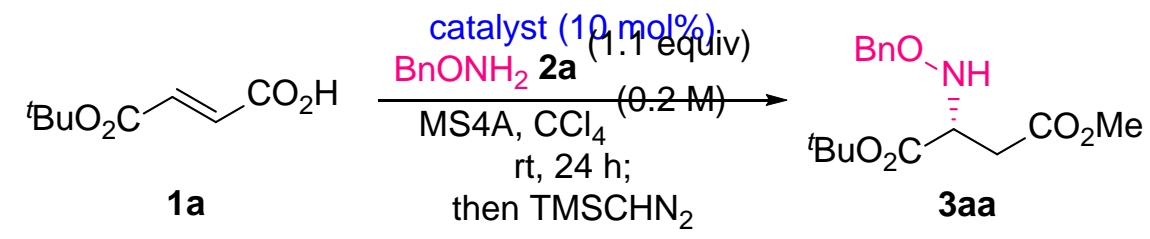

\begin{tabular}{cccc}
\hline entry & catalyst & 3aa (\%) & ee (\%) \\
\hline $\mathbf{1}$ & $\boldsymbol{A}$ & $\mathbf{8 0}$ & $\mathbf{8 8}$ \\
2 & $\mathbf{B}$ & 66 & 80 \\
3 & $\mathbf{C}$ & 72 & 76 \\
4 & $\mathbf{D}$ & 59 & 90 \\
5 & $\mathbf{E}$ & 62 & 80 \\
6 & $\mathbf{F}$ & 54 & 51 \\
\hline
\end{tabular}
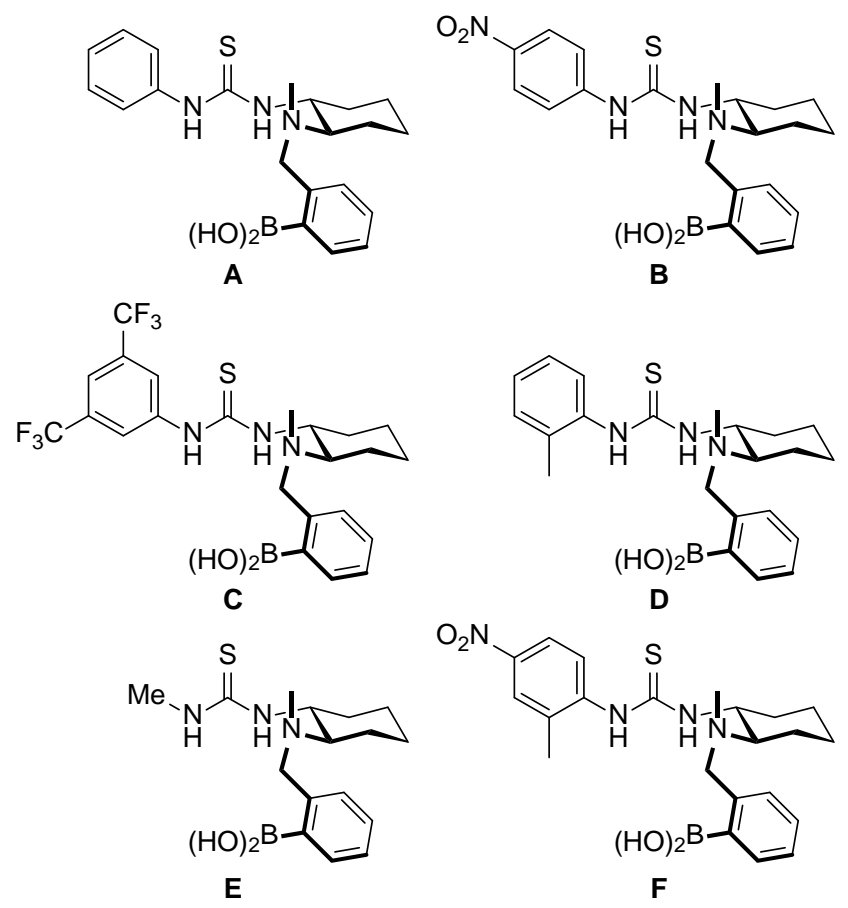
Table S3. Screening of Acid Additives

\begin{tabular}{|c|c|c|c|c|}
\hline \multirow[t]{8}{*}{$1 a$} & \multicolumn{2}{|c|}{ 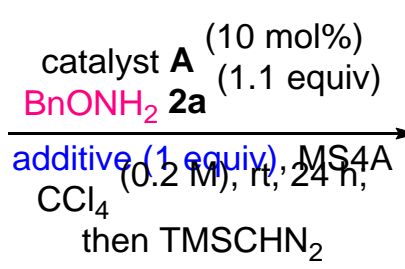 } & \multicolumn{2}{|c|}{$\mathrm{uO}_{2} \mathrm{C} \overbrace{3 \mathbf{3}}^{\mathrm{Bna}_{-}^{\mathrm{NH}}}$} \\
\hline & entry & additive & Заa (\%) & ee (\%) \\
\hline & 1 & none & 80 & 88 \\
\hline & 2 & $\mathrm{HCO}_{2} \mathrm{H}$ & 0 & - \\
\hline & 3 & $\mathrm{MeCO}_{2} \mathrm{H}$ & 57 & 91 \\
\hline & 4 & ${ }^{t} \mathrm{BuCO}_{2} \mathrm{H}$ & 77 & 87 \\
\hline & 5 & $\mathrm{PhCO}_{2} \mathrm{H}$ & 76 & 93 \\
\hline & 6 & $\mathrm{TsOH} \cdot \mathrm{H}_{2} \mathrm{O}$ & 0 & - \\
\hline
\end{tabular}

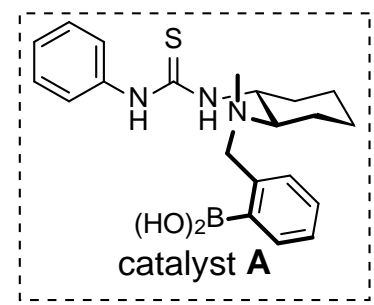

Table S4. Investigation of Nucleophiles

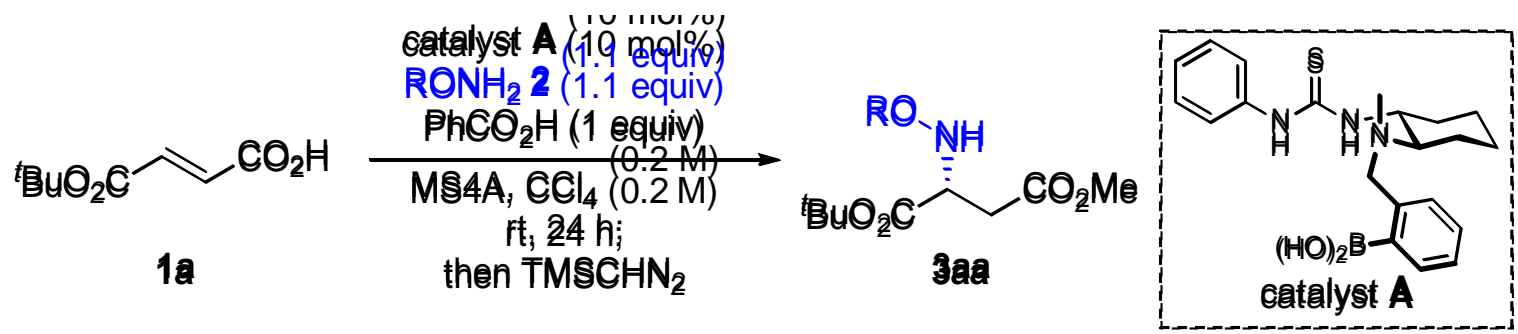

\begin{tabular}{cccc}
\hline entry & nucleophile & 3aa (\%) & ee (\%) \\
\hline 1 & 2a & 76 & 93 \\
2 & 2b & 50 & 74 \\
3 & 2c & 64 & 96 \\
4 & 2d & 60 & 87 \\
$5^{a}$ & 2e & 70 & 85 \\
6 & S2a & 0 & - \\
\hline
\end{tabular}

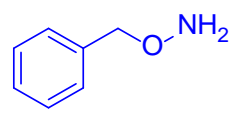

$2 a$

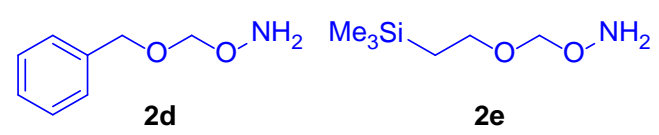

$2 e$<smiles>NOCc1ccc(C(F)(F)F)cc1</smiles>

2b<smiles>NOC(=O)c1ccccc1</smiles>

${ }^{a}$ Ee was determined after $N$-benzoylation.

Figure S1. Unsuccessful Substrates

Monomethyl fumarate (S1a) and fumaryl monoanilide (S1b) did not undergo the aza-Michael addition, probably due to low solubility in $\mathrm{CCl}_{4}$.<smiles>COC(=O)C=CC(=O)O</smiles>

S1a<smiles>O=C(O)/C=C/C(=O)Nc1ccccc1</smiles>

S1b 


\section{(A-2) Determination of Stereochemistry}

The aza-Michael adduct 3aa was converted into $N$-Fmoc-aspartic diester S3aa and the stereochemistry was determined as $R$ configuration by comparison of HPLC charts and optical rotations with ( $S$ )- $N$-Fmocaspartic diester derived from the commercially available mono-tert-butyl-L-aspartate (Scheme S1).

Scheme S1

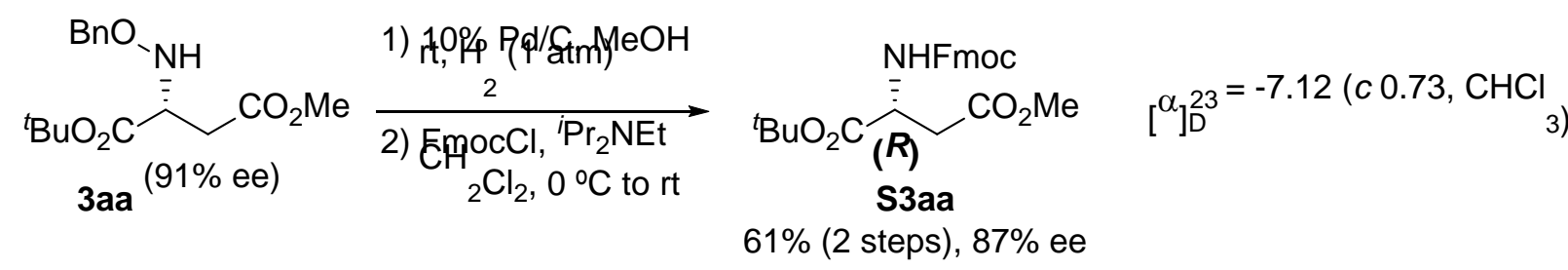

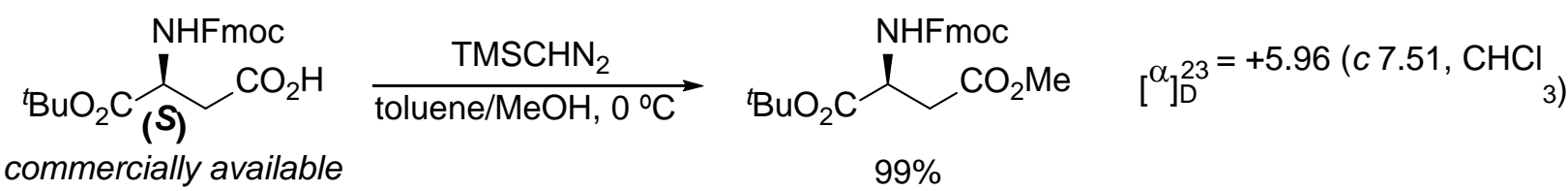




\section{(B) General}

All manipulations were carried out under argon atmosphere unless otherwise noted. Infrared (IR) spectra were recorded on a JASCO FT/IR-4100 Fourier transform infrared spectrophotometer. NMR spectra were recorded on a JEOL ECP-400 spectrometer and JEOL ECA-500 spectrometer, operating at $400 \mathrm{MHz}\left({ }^{1} \mathrm{H}\right)$ or $100 \mathrm{MHz}\left({ }^{13} \mathrm{C}\right)$ and $500 \mathrm{MHz}\left({ }^{1} \mathrm{H}\right)$ or $125 \mathrm{MHz}\left({ }^{13} \mathrm{C}\right)$, respectively. Chemical shifts in $\mathrm{CDCl}_{3}$, DMSO$d_{6}$, and $\mathrm{CD}_{3} \mathrm{OD}$ were reported in the scale relative to $\mathrm{CHCl}_{3}(7.26 \mathrm{ppm})$, DMSO (2.50 ppm), and $\mathrm{MeOH}$ (3.31 ppm) for ${ }^{1} \mathrm{H} \mathrm{NMR}$, and to $\mathrm{CDCl}_{3}\left(77.0 \mathrm{ppm}\right.$ ) for ${ }^{13} \mathrm{C}$ NMR as internal references, respectively. NMR data are reported as follows: chemical shifts, multiplicity (s: singlet, d: doublet, t: triplet, q: quartet, quin: quintet, m: multiplet, br: broad signal), coupling constant (Hz), and integration. ESI-HRMS spectra were measured on a Shimadzu LCMS-IT-TOF fitted with an ESI. Optical rotations were measured on a JASCO P-2200 digital polarimeter with a path length of $1 \mathrm{~cm}$; concentrations are quoted in grams per $100 \mathrm{~mL}$. $[\alpha]_{D}$ values are measured in $10^{-1} \mathrm{deg} \mathrm{cm}^{2} / \mathrm{g}$. Chiral HPLC analyses were carried out using a SHIMADZU DGU-20A 5 . Column chromatography was performed with Cica silica gel 60N (40-100 $\mu \mathrm{m}$, spherical, neutral). Dry solvents were purchased from Wako Pure Chemical Industries, Ltd. and used as received. Organocatalysts A-F were prepared according to our developed procedures. ${ }^{1}$ 
(C) Materials and Methods

\section{(C-1) Preparation of Substrates}

Monoethyl fumarate (1c) was purchased from Tokyo Chemical Industry Co., Ltd. Monomethyl fumarate (S1a) was purchased from Sigma-Aldrich Co. LLC. mono-tert-butyl fumarate $(\mathbf{1 a})^{2}$ and (E)-4-oxo-4(phenylamino)but-2-enoic acid (S1b) ${ }^{3}$ were prepared according to the reported procedures.

Mono-tert-butyl fumarate (1a) ${ }^{2}$ : White solids. ${ }^{t} \mathrm{BuO}_{2} \mathrm{C} \curvearrowright \mathrm{CO}_{2} \mathrm{H} \quad{ }^{1} \mathrm{H}$ NMR $\left(400 \mathrm{MHz}, \mathrm{CDCl}_{3}\right) \delta: 6.87(\mathrm{~d}, J=15.6 \mathrm{~Hz}, 1 \mathrm{H}), 6.76(\mathrm{~d}, J=15.6 \mathrm{~Hz}, 1 \mathrm{H})$, $1 \mathrm{a}$ $1.52(\mathrm{~s}, 9 \mathrm{H}) \mathrm{ppm}$.

(E)-4-Oxo-4-(phenylamino)but-2-enoic acid (S1b) ${ }^{3}$ : White solids.

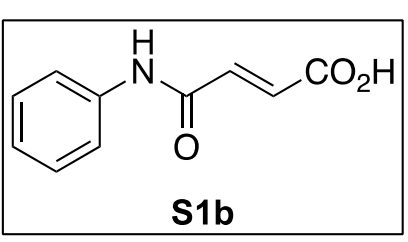
IR (neat) $\tilde{v}:$ 1696, $1654 \mathrm{~cm}^{-1} ;{ }^{1} \mathrm{H}$ NMR (400 MHz, DMSO-d 6 ) $\delta: 12.99$ (br s, 1H), 10.51 (s, 1H), 7.67 (d, $J=8.0 \mathrm{~Hz}, 2 \mathrm{H}), 7.34$ (t, $J=7.6 \mathrm{~Hz}, 2 \mathrm{H}), 7.14$ (d, $J$ $=15.2 \mathrm{~Hz}, 1 \mathrm{H}), 7.09(\mathrm{t}, J=7.6 \mathrm{~Hz}, 1 \mathrm{H}), 6.65(\mathrm{~d}, J=15.2 \mathrm{~Hz}, 1 \mathrm{H}) \mathrm{ppm} ;{ }^{13} \mathrm{C}$ NMR (100 MHz, DMSO- $\left.d_{6}\right) \delta: 166.4,161.6,138.6,137.2,130.8,128.9,124.0$, 119.4 ppm; HRMS (ESI) m/z calcd. for [M-H]': 190.0510, found: 190.0521.

Monobenzyl fumarate (1b) ${ }^{4}$ : To a solution of benzyl tert-butyl fumarate ${ }^{5}$ (262.1 mg, 1.0 mmol, 1 equiv) $\mathrm{BnO}_{2} \mathrm{C} \underset{\mathbf{1 b}}{\sim} \mathrm{CO}_{2} \mathrm{H}$ in $\mathrm{CH}_{2} \mathrm{Cl}_{2}(4.0 \mathrm{~mL})$ was added TFA $(1.8 \mathrm{~mL})$ and stirred at room temperature for 7 h. The mixture was concentrated, and the resulting solids were recrystallised from $\mathrm{CH}_{2} \mathrm{Cl}_{2}$ and hexane to afford $\mathbf{1 b}$ as white solids (138.4 $\mathrm{mg}, 0.67 \mathrm{mmol}, 67 \%$ ).

IR (neat) $\tilde{v}$ : 2940, 1719, $1694 \mathrm{~cm}^{-1}$; ${ }^{1} \mathrm{H}$ NMR (400 MHz, $\left.\mathrm{CDCl}_{3}\right) \delta: 7.39-7.34$ (m, 5H), 6.99 (d, $J=16.0$ Hz, 1H), 6.88 (d, $J=16.0 \mathrm{~Hz}, 1 \mathrm{H}), 5.25$ (s, 2H) ppm.

\section{Ethyl (S,E)-4-((1-(tert-butoxy)-1-oxo-3-phenylpropan-2-yl)amino)-4-oxobut-2-enoate (S1d):}

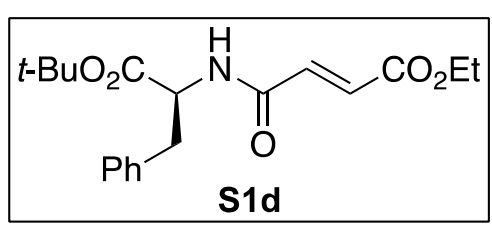

A mixture of L-phenylalanine tert-butyl ester hydrochloride ${ }^{6}$ (1.32 g, 6.0 mmol, 1.0 equiv), monoethyl fumarate (944.0 mg, $6.6 \mathrm{mmol}, 1.1$ equiv), HOBt (972.0 mg, 7.2 mmol, 1.2 equiv), Et $3 \mathrm{~N}$ (1.82 g, 18.0 mmol, 3.0 equiv) and EDCI (1.38 g, 7.2 mmol, 1.2 equiv) in DMF (30 mL) was stirred at room temperature for $20 \mathrm{~h}$. The solution was diluted with brine $(20 \mathrm{~mL})$ and extracted with $\mathrm{Et}_{2} \mathrm{O}(20 \mathrm{~mL}$, 2 times). The combined organic phase was dried over $\mathrm{Na}_{2} \mathrm{SO}_{4}$ followed by filtration and concentration under reduced pressure. The residue was then purified by silica-gel column chromatography (eluent: hexane/EtOAc, 3:1) to afford S1d as white solids (1.43 g, $4.12 \mathrm{mmol}, 67 \%)$.

m.p. 139.3-140.0 ${ }^{\circ} \mathrm{C} ; \quad[\alpha]_{D}^{19} 106.9$ (c 0.89, $\mathrm{CHCl}_{3}$ ); IR (neat) $\tilde{v}: 3312,1734,1716,1637 \mathrm{~cm}^{-1} ;{ }^{1} \mathrm{H} \mathrm{NMR}$ $\left(500 \mathrm{MHz}, \mathrm{CDCl}_{3}\right) \delta: 7.29-7.22(\mathrm{~m}, 3 \mathrm{H}), 7.14(\mathrm{~d}, J=6.0 \mathrm{~Hz}, 2 \mathrm{H}), 6.92(\mathrm{~d}, J=15.5 \mathrm{~Hz}, 1 \mathrm{H}), 6.80(\mathrm{~d}, J=$ $15.5 \mathrm{~Hz}, 1 \mathrm{H}), 6.45$ (d, $J=7.5 \mathrm{~Hz}, 1 \mathrm{H}), 4.84$ (dt, $J=7.0,6.0 \mathrm{~Hz}, 1 \mathrm{H}), 4.22$ (q, $J=7.5 \mathrm{~Hz}, 2 \mathrm{H}), 3.14$ (d, $J=$ $6.0 \mathrm{~Hz}, 2 \mathrm{H}), 1.42$ (s, 9H), 1.30 (t, $J=7.5 \mathrm{~Hz}, 3 \mathrm{H}),{ }^{13} \mathrm{C}$ NMR (125 MHz, $\left.\mathrm{CDCl}_{3}\right) \delta: 170.2,165.5,163.0$, 135.9, 130.9, 129.6, 128.5, 127.2, 82.9, 61.3, 53.9, 37.9, 28.0, 14.2 ppm; HRMS (ESI) m/z calcd. for $\mathrm{C}_{19} \mathrm{H}_{25} \mathrm{NO}_{5}[\mathrm{M}+\mathrm{Na}]^{+}:$370.1625, found: 370.1588.

(S,E)-4-((1-(tert-Butoxy)-1-oxo-3-phenylpropan-2-yl)amino)-4-oxobut-2-enoic acid (1d): To a solution 
of S1d (1.43 g, $4.12 \mathrm{mmol}, 1.0$ equiv) in THF (20 mL) and water (8.0 mL) was added LiOH (172.8 mg,

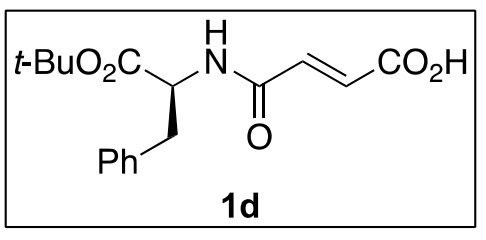

$4.12 \mathrm{mmol}, 1.0$ equiv) and stirred at room temperature for $12 \mathrm{~h}$. The mixture was washed with $\mathrm{Et}_{2} \mathrm{O}$, and the aqueous phase was acidified with $1 \mathrm{M} \mathrm{HCl}$ aq. The solution was extracted with EtOAc $(20 \mathrm{~mL}, 2$ times) and the combined organic layer was washed with brine. After drying over $\mathrm{Na}_{2} \mathrm{SO}_{4}$

followed by filtration, the solvent wasremoved under reduced pressure to afford $\mathbf{1 d}$ as white solids (565.3 mg, $1.77 \mathrm{mmol}, 43 \%)$.

m.p. 118.7-119.1 ${ }^{\circ} \mathrm{C} ; \quad[\alpha]_{D}^{20} 105.8$ (c 0.68, $\mathrm{CHCl}_{3}$ ); IR (neat) $\tilde{v}: 3350,1726,1659,1642 \mathrm{~cm}^{-1} ;{ }^{1} \mathrm{H} \mathrm{NMR}(500$ $\left.\mathrm{MHz} \mathrm{CDCl}_{3}\right) \delta:$ 7.29-7.24 (m, $\left.3 \mathrm{H}\right), 7.15-7.14(\mathrm{~m}, 2 \mathrm{H}), 7.00(\mathrm{~d}, J=15.5 \mathrm{~Hz}, 1 \mathrm{H}), 6.83(\mathrm{~d}, J=7.5 \mathrm{~Hz}, 1 \mathrm{H})$, $6.82(\mathrm{~d}, J=15.0 \mathrm{~Hz}, 1 \mathrm{H}), 4.89$ (dt, $J=8.0,7.0 \mathrm{~Hz}, 1 \mathrm{H}), 3.13$ (d, $J=6.0 \mathrm{~Hz}, 2 \mathrm{H}), 1.42(\mathrm{~s}, 9 \mathrm{H}) \mathrm{ppm} ;{ }^{13} \mathrm{C}$ NMR (125 MHz, $\left.\mathrm{CDCl}_{3}\right) \delta:$ 170.9, 169.4, 163.1, 137.6, 135.7, 130.3, 129.6, 128.6, 127.3, 83.4, 54.0, 38.1, 28.0 ppm; HRMS (ESI) $\mathrm{m} / \mathrm{z}$ calcd. for $\mathrm{C}_{17} \mathrm{H}_{21} \mathrm{NO}_{5}[\mathrm{M}+\mathrm{Na}]^{+}$: 342.1312, found: 342.1295.

\section{Ethyl (R,E)-4-((1-(tert-butoxy)-1-oxo-3-phenylpropan-2-yl)amino)-4-oxobut-2-enoate (S1e):}

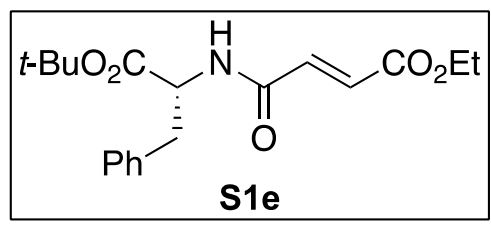

A mixture of D-phenylalanine $t$-butyl ester hydrochloride ${ }^{6}(1.32 \mathrm{~g}, 6.0$ mmol, 1.0 equiv), monoethyl fumarate (944.0 mg, $6.6 \mathrm{mmol}, 1.1$ equiv), HOBt (972.0 mg, 7.2 mmol, 1.2 equiv), Et $3 \mathrm{~N}$ (1.82 g, 18.0 mmol, 3.0 equiv) and EDCI (1.38 g, $7.2 \mathrm{mmol}, 1.2$ equiv) in DMF (30 mL) was stirred at room temperature for $20 \mathrm{~h}$. The solution was diluted with brine $(20 \mathrm{~mL})$ and extracted with $\mathrm{Et}_{2} \mathrm{O}(20 \mathrm{~mL}$, 2 times). The combined organic phase was dried over $\mathrm{Na}_{2} \mathrm{SO}_{4}$ followed by filtration and concentration under reduced pressure. The residue was then purified by silica-gel column chromatography (eluent: hexane/EtOAc, 3:1) to afford S1d as white solids (1.46 g, $4.23 \mathrm{mmol}, 70 \%)$.

m.p. 135.5-137.8 ${ }^{\circ} \mathrm{C}$; $[\alpha]_{D}^{18}$-91.3 (c 0.94, $\mathrm{CHCl}_{3}$ ); IR (neat) $\tilde{v}: 3310,1734,1716,1636 \mathrm{~cm}^{-1}$; ${ }^{1} \mathrm{H}$ NMR $\left(500 \mathrm{MHz}, \mathrm{CDCl}_{3}\right) \delta: 7.27-7.22(\mathrm{~m}, 3 \mathrm{H}), 7.15-7.13(\mathrm{~m}, 2 \mathrm{H}), 6.94$ (d, $\left.J=15.5 \mathrm{~Hz}, 1 \mathrm{H}\right), 6.81$ (d, $J=14.5$ Hz, 1H), 6.54 (d, $J=7.5 \mathrm{~Hz}, 1 \mathrm{H}$ ), 4.85 (dt, $J=7.5,6.0 \mathrm{~Hz}, 1 \mathrm{H}), 4.21$ (q, $J=7.0 \mathrm{~Hz}, 2 \mathrm{H}), 3.14$ (d, $J=6.0$ $\mathrm{Hz}, 2 \mathrm{H}), 1.41$ (s, 9H), 1.29 (t, $J=7.5 \mathrm{~Hz}, 3 \mathrm{H}),{ }^{13} \mathrm{C} \mathrm{NMR}\left(125 \mathrm{MHz}, \mathrm{CDCl}_{3}\right) \delta: 170.3,165.5,163.0,135.95$, 135.91, 130.9, 129.5, 128.5, 127.1, 82.8, 61.3, 53.9, 37.9, 28.0, 14.2 ppm; HRMS (ESI) m/z calcd. for $\mathrm{C}_{19} \mathrm{H}_{25} \mathrm{NO}_{5}[\mathrm{M}+\mathrm{Na}]^{+}:$370.1625, found: 370.1576.

(R,E)-4-((1-(tert-Butoxy)-1-oxo-3-phenylpropan-2-yl)amino)-4-oxobut-2-enoic acid (1e): To a solution

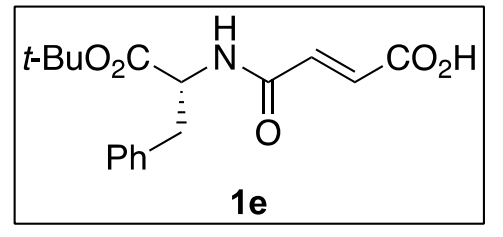
of S1e (1.46 g, $4.23 \mathrm{mmol}, 1.0$ equiv) in THF $(20 \mathrm{~mL})$ and water $(8.0 \mathrm{~mL})$ was added $\mathrm{LiOH}$. (177.4 $\mathrm{mg}, 4.23 \mathrm{mmol}, 1.0$ equiv) and stirred at room temperature for $12 \mathrm{~h}$. The mixture was washed with $\mathrm{Et}_{2} \mathrm{O}$, and the aqueous phase was acidified with $1 \mathrm{M} \mathrm{HCl}$ aq. The solution was extracted with EtOAc (20 mL, 2 times) and the combined organic layer was washed with brine. After drying over $\mathrm{Na}_{2} \mathrm{SO}_{4}$ followed by filtration, the solvent was removed under reduced pressure to afford $\mathbf{1 e}$ as white solids (855.0 mg, $2.67 \mathrm{mmol}, 65 \%)$.

m.p. 118.6-119.2 ${ }^{\circ} \mathrm{C} ; \quad[\alpha]_{D}^{21}-95.0$ (c 0.48, $\mathrm{CHCl}_{3}$ ); IR (neat) $\tilde{v}$ : 3351, 1726, 1660, 1642 $\mathrm{cm}^{-1} ;{ }^{1} \mathrm{H}$ NMR (500 $\left.\mathrm{MHz}, \mathrm{CDCl}_{3}\right) \delta: 7.29-7.24$ (m, 3 H), 7.14 (d, $\left.J=7.0 \mathrm{~Hz}, 2 \mathrm{H}\right), 6.99$ (d, $\left.J=15.5 \mathrm{~Hz}, 1 \mathrm{H}\right), 6.81(\mathrm{~d}, J=15.0$ Hz, 1H), 6.74 (d, $J=8.0 \mathrm{~Hz}, 1 \mathrm{H}$ ), 4.88 (dt, $J=8.0,6.0 \mathrm{~Hz}, 1 \mathrm{H}), 3.14$ (d, $J=6.5 \mathrm{~Hz}, 2 \mathrm{H}), 1.42$ (s, 9H) ppm;

${ }^{13} \mathrm{C}$ NMR (125 MHz, $\left.\mathrm{CDCl}_{3}\right) \delta: 170.9,169.4,163.0,137.6,130.2,129.6,128.6,127.3,83.4,54.0$, 38.0, 
28.0 ppm; HRMS (ESI) m/z calcd. for $\mathrm{C}_{17} \mathrm{H}_{21} \mathrm{NO}_{5}[\mathrm{M}+\mathrm{Na}]^{+}:$342.1312, found: 342.1291.

Ethyl (E)-4-((tert-butoxycarbonylmethyl)amino)-4-oxobut-2-enoate (S1f): A mixture of glycine tert-

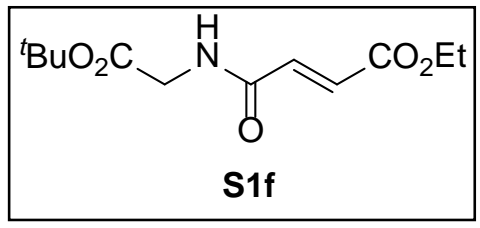
butyl ester hydrochloride (835.0 mg, $4.98 \mathrm{mmol}, 1.0$ equiv), monoethyl fumarate (788.4 mg, $5.47 \mathrm{mmol}, 1.1$ equiv), HOBt (1.01 g, $7.47 \mathrm{mmol}, 1.5$ equiv), and EDCI (1.43 g, $7.47 \mathrm{mmol}, 1.5$ equiv) in DMF (13.5 mL) was stirred at room temperature for $20 \mathrm{~h}$. The solution was diluted with brine (30 $\mathrm{mL}$ ) and extracted with $\mathrm{Et}_{2} \mathrm{O}$ (50 mL, 3 times). The combined organic phase was dried over $\mathrm{Na}_{2} \mathrm{SO}_{4}$ followed by filtration and concentration under reduced pressure. The residue was then purified by silicagel column chromatography (eluent: hexane/EtOAc, 1:1) to afford S1f as yellow oil (716.7 mg, $2.78 \mathrm{mmol}$, $56 \%)$.

IR (neat) $\tilde{v}: 3301,2980,1724,1668 \mathrm{~cm}^{-1} ;{ }^{1} \mathrm{H}$ NMR (500 MHz, $\left.\mathrm{CDCl}_{3}\right) \delta: 6.94$ (d, $\left.J=15.0 \mathrm{~Hz}, 1 \mathrm{H}\right), 6.82$ (d, $J=15.0 \mathrm{~Hz}, 1 \mathrm{H}), 4.24$ (q, $J=7.0 \mathrm{~Hz}, 2 \mathrm{H}), 4.03$ (d, $J=4.5 \mathrm{~Hz}, 2 \mathrm{H}), 1.47$ (s, 9H), 1.31 (t, $J=6.5 \mathrm{~Hz}, 3 \mathrm{H}$ ) ppm; ${ }^{13} \mathrm{C}$ NMR (125 MHz, $\left.\mathrm{CDCl}_{3}\right) \delta: 168.6,165.5,163.6,135.5,131.1,82.9,61.3,42.4,28.1,14.2$ ppm; HRMS (ESI) $\mathrm{m} / \mathrm{z}$ calcd. for $[\mathrm{M}+\mathrm{Na}]^{+}:$280.1155, found: 280.1157.

(E)-4-((tert-Butoxycarbonylmethyl)amino)-4-oxobut-2-enoic acid (1f): A solution of S1f (716.0 mg, ${ }^{t} \mathrm{BuO}_{2} \mathrm{C}{ }_{\substack{\mathrm{Of} \\ \mathrm{O}}}^{\mathrm{H}} \mathrm{CO}_{2} \mathrm{H}$ $2.78 \mathrm{mmol}, 1$ equiv) in THF (14 mL) was treated with $1 \mathrm{M} \mathrm{LiOH}$ aq. (2.78 $\mathrm{mL}, 2.78 \mathrm{mmol}, 1.0$ equiv) and stirred at ambient temperature for $4 \mathrm{~h}$. The mixture was acidified with $1 \mathrm{M} \mathrm{HCl}$ aq. and extracted with $\mathrm{CHCl}_{3}(30 \mathrm{~mL}, 3$ times). After drying over $\mathrm{Na}_{2} \mathrm{SO}_{4}$ followed by filtration, the solvent was removed under reduce pressure to afford $\mathbf{1 f}$ as white solids (366.7 $\mathrm{mg}, 1.60 \mathrm{mmol}, 60 \%$ ).

m.p. $229.4{ }^{\circ} \mathrm{C}$ (decomp.); IR (neat) $\tilde{v}$ : 3339, 2868, 1732, 1689, 1658, $1637 \mathrm{~cm}^{-1}$; ${ }^{1} \mathrm{H}$ NMR (500 MHz, $\left.\mathrm{CDCl}_{3}\right) \delta: 7.02$ (d, $\left.J=15.5 \mathrm{~Hz}, 1 \mathrm{H}\right), 6.84$ (d, $\left.J=15.5 \mathrm{~Hz}, 1 \mathrm{H}\right), 6.54$ (br s, 1H), 4.06 (d, $\left.J=4.5 \mathrm{~Hz}, 2 \mathrm{H}\right)$, 1.48 (s, 9H) ppm; ${ }^{13} \mathrm{C}$ NMR (125 MHz, $\left.\mathrm{CD}_{3} \mathrm{OD}\right) \delta$ : 168.6, 166.9, 165.3, 135.6, 130.5, 81.7, 41.6, 26.9 ppm; HRMS (ESI) $m / z$ calcd. for [M-H]': 228.0877, found: 228.0868.

Ethyl (S,E)-4-((1,3-bis(tert-butoxy)-1-oxopropan-2yl)amino)-4-oxobut-2-enoate (S1g): A mixture of

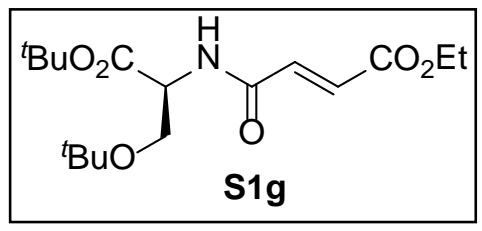
O-tert-butyl-L-serine tert-butyl ester hydrochloride (2.53 g, $10.0 \mathrm{mmol}, 1.0$ equiv), monoethyl fumarate (1.72 g, $12.0 \mathrm{mmol}, 1.2$ equiv), HOBt (1.62 g, 12.0 mmol, 1.2 equiv), and EDCI (2.87 g, 15.0 mmol, 1.5 equiv) in DMF (27 $\mathrm{mL}$ ) was stirred at room temperature for $12 \mathrm{~h}$. The solution was diluted with brine $(50 \mathrm{~mL})$ and extracted with $\mathrm{Et}_{2} \mathrm{O}$ (50 mL, 3 times). The combined organic phase was dried over $\mathrm{Na}_{2} \mathrm{SO}_{4}$ followed by filtration and concentration under reduced pressure. The residue was then purified by silica-gel column chromatography (eluent: hexane/EtOAc, 1:1) to afford S1g as white solids (3.06 g, 8.91 mmol, 89\%).

m.p. 79.4- $80.5{ }^{\circ} \mathrm{C}$; $[\alpha]_{D}^{25} 43.2$ (c 0.45, $\mathrm{CHCl}_{3}$ ); IR (neat) $\tilde{v}: 3309,2981,1741,1715,1656 \mathrm{~cm}^{-1} ;{ }^{1} \mathrm{H}$ NMR $\left(125 \mathrm{MHz} \mathrm{CDCl}_{3}\right) \delta: 6.99(\mathrm{~d}, J=15.5 \mathrm{~Hz}, 1 \mathrm{H}), 6.96(\mathrm{~d}, J=8.5 \mathrm{~Hz}, 1 \mathrm{H}), 4.60$ (dt, $\left.J=8.0,3.0 \mathrm{~Hz}, 1 \mathrm{H}\right)$, 4.13 (q, $J=7.0 \mathrm{~Hz}, 2 \mathrm{H}$ ), 3.69 (dd, $J=8.0,3.0 \mathrm{~Hz}, 1 \mathrm{H}$ ), 3.46 (dd, $J=8.0,3.0 \mathrm{~Hz}, 1 \mathrm{H}), 1.35$ (s, $9 \mathrm{H}), 1.18$ (t, $J=7.0 \mathrm{~Hz}, 3 \mathrm{H}), 1.01$ (s, 9H) ppm; ${ }^{13} \mathrm{C}$ NMR (125 MHz, $\left.\mathrm{CDCl}_{3}\right) \delta: 169.0,165.5,163.2,136.3,130.5,81.9$, 73.0, 62.1, 61.1, 53.5, 27.9, 27.2, 14.1 ppm; HRMS (ESI) $\mathrm{m} / \mathrm{z}$ calcd. for $\mathrm{C}_{17} \mathrm{H}_{29} \mathrm{NO}_{6}[\mathrm{M}+\mathrm{Na}]^{+}$: 366.1887, 
found: 366.1861.

(S,E)-4-((1,3-Bis(tert-butoxy)-1-oxopropan-2-yl)amino)-4-oxobut-2-enoic acid (1g): A solution of S1g

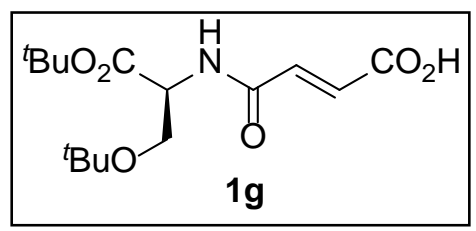

(1.03 g, $3.0 \mathrm{mmol}, 1.0$ equiv) in THF (15 mL) was treated with $1 \mathrm{M} \mathrm{LiOH}$ aq. (3.0 mL, $3.0 \mathrm{mmol}, 1.0$ equiv) and stirred at ambient temperature for $4 \mathrm{~h}$. The mixture was acidified with $1 \mathrm{M} \mathrm{HCl}$ aq. and extracted with $\mathrm{CHCl}_{3}(20 \mathrm{~mL}, 3$ times). After drying over $\mathrm{Na}_{2} \mathrm{SO}_{4}$ followed by filtration, the solvent was removed to afford $\mathbf{1 g}$ as white solids (836.9 $\mathrm{mg}, 2.65 \mathrm{mmol}, 88 \%)$.

m.p. 165.5-167.9 ${ }^{\circ}$; $\quad[\alpha]_{D}^{23} 25.6$ (c 0.42, $\mathrm{CHCl}_{3}$ ); IR (neat) $\tilde{v}: 3331,3074,1720,1706,1631 \mathrm{~cm}^{-1} ;{ }^{1} \mathrm{H} \mathrm{NMR}$ $\left(500 \mathrm{MHz}, \mathrm{CDCl}_{3}\right) \delta: 7.19(\mathrm{~d}, J=8.5 \mathrm{~Hz}, 1 \mathrm{H}), 7.07$ (d, $\left.J=15.5 \mathrm{~Hz}, 1 \mathrm{H}\right), 6.88(\mathrm{~d}, J=15.5 \mathrm{~Hz}, 1 \mathrm{H}), 4.73$ (dt, $J=9.0,3.0 \mathrm{~Hz}, 1 \mathrm{H}$ ), 3.80 (dd, $J=9.0,3.0 \mathrm{~Hz}, 1 \mathrm{H}$ ), 3.56 (dd, $J=9.0,3.0 \mathrm{~Hz}, 1 \mathrm{H}$ ), 1.45 (s, $9 \mathrm{H}$ ), 1.12 (s, 9H) ppm; ${ }^{13} \mathrm{C}$ NMR (125 MHz, $\left.\mathrm{CDCl}_{3}\right) \delta:$ 169.7, 168.8, 137.3, 130.5, 82.8, 73.5, 62.2, 53.6, 28.0, 27.3 ppm; HRMS (ESI) m/z calcd. for $\mathrm{C}_{15} \mathrm{H}_{25} \mathrm{NO}_{6}[\mathrm{M}+\mathrm{Na}]^{+}$: 338.1574, found: 338.1554.

\section{(C-2) Preparation of Nucleophiles}

O-benzylhydroxylamine (2a) was prepared by neutralization of $\mathrm{BnONH}_{2} \cdot \mathrm{HCl}$ by $4 \mathrm{M} \mathrm{NaOH}$ aq. After extraction with $\mathrm{CHCl}_{3}$, general work up and dried under vacuum to afford 2a. $O$-Benzoylhydroxylamine (S2a) was prepared according to the reported procedure. ${ }^{8}$

O-(4-Methoxybenzyl)hydroxylamine (2b): A mixture of $N$-hydroxyphthalimide (1.80 g, 11.0 mmol, 1.1

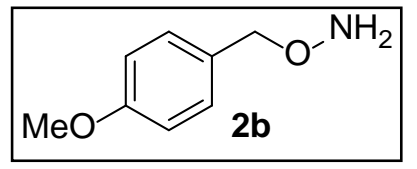
equiv), 4-methoxybenzyl chloride (1.86 g, 10.0 mmol, 1.0 equiv), and $\mathrm{Et}_{3} \mathrm{~N}$ (1.22 g, $12.1 \mathrm{mmol}, 1.1$ equiv) in $\mathrm{CH}_{2} \mathrm{Cl}_{2}(25 \mathrm{~mL})$ was stirred at room temperature for $4 \mathrm{~h}$. The suspension was washed with brine (20 mL, 3 times) and the combined organic phase was dried over $\mathrm{Na}_{2} \mathrm{SO}_{4}$. After the solvent was removed under reduced pressure, the residue was dissolved in $\mathrm{CHCl}_{3} / \mathrm{MeOH}=3: 1(50 \mathrm{~mL})$. To the solution was added to $\mathrm{N}_{2} \mathrm{H}_{4} \cdot \mathrm{H}_{2} \mathrm{O}(750.9 \mathrm{mg}, 15.0$ mmol, 1.5 equiv) and stirred at room temperature for $2 \mathrm{~h}$. The mixture was filtered through a pad of Celite ${ }^{\circledR}$ and the filtrate was concentrated under reduced pressure. The residue was purified by silica-gel column chromatography (eluent: hexane/EtOAc, 1:1) to afford $\mathbf{2 b}$ as colorless oil (1.10 g, $6.2 \mathrm{mmol}, 62 \%)$. IR (neat) $\tilde{v}: 2977,2917 \mathrm{~cm}^{-1} ;{ }^{1} \mathrm{H}$ NMR $\left(500 \mathrm{MHz}, \mathrm{CDCl}_{3}\right) \delta: 7.29$ (d, $\left.J=9.0 \mathrm{~Hz}, 2 \mathrm{H}\right), 6.89$ (d, $J=9.0 \mathrm{~Hz}$, 2H), 5.33 (br s, 2H), 4.61 (s, 2H), 3.80 (s, 3H) ppm; ${ }^{13} \mathrm{C}$ NMR (125 MHz, $\mathrm{CDCl}_{3}$ ) $\delta: 159.5,130.1,129.4$, 113.9,113.7, 77.7, 53.3 ppm; HRMS (ESI) m/z calcd. for $\mathrm{C}_{8} \mathrm{H}_{9} \mathrm{NO}$, [M] ${ }^{+}:$176.0682, found: 176.0556 .

O-(4-Trifluoromethylbenzyl)hydroxylamine (2c): 2c was prepared through the procedure analogous to

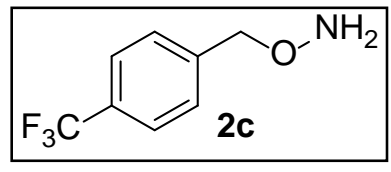
that of 2b. Alkylation was performed using $N$-hydroxyphthalimide (1.35 g, 7.0 mmol, 1.4 equiv), 4-(trifluoromethyl)benzyl chloride (815.0 mg, $5.0 \mathrm{mmol}, 1.0$ equiv), and $\mathrm{Et}_{3} \mathrm{~N}$ (708.3 mg, $7.0 \mathrm{mmol}, 1.4$ equiv) in $\mathrm{CH}_{2} \mathrm{Cl}_{2}(20 \mathrm{~mL})$. Deprotection of phthalimide was conducted with $\mathrm{N}_{2} \mathrm{H}_{4} \cdot \mathrm{H}_{2} \mathrm{O}$ (525.6 mg, $10.5 \mathrm{mmol}, 1.5$ equiv) in $\mathrm{CH}_{3} \mathrm{Cl} / \mathrm{MeOH}=3: 1$ (15 mL), which afforded 2c as colorless oil (810.0 mg, $4.2 \mathrm{mmol}, 84 \%)$.

IR (neat) $\tilde{v}: 2940,1323 \mathrm{~cm}^{-1} ;{ }^{1} \mathrm{H}$ NMR $\left(500 \mathrm{MHz}, \mathrm{CDCl}_{3}\right) \delta: 7.63(\mathrm{~d}, J=8.0 \mathrm{~Hz}, 2 \mathrm{H}), 7.48$ (d, $J=8.0 \mathrm{~Hz}$, 2H), 5.48 (br s, 2H), 4.75 (s, 2H) ppm; ${ }^{13} \mathrm{C}$ NMR (125 MHz, $\mathrm{CDCl}_{3}$ ) $\delta: 141.7,130.0$ (q, $J=32.5 \mathrm{~Hz}$ ), 128.3, 
125.4 (q, $J=3.9 \mathrm{~Hz}$ ), 76.9 ppm; HRMS (ESI) m/z calcd. for $\mathrm{C}_{8} \mathrm{H}_{8} \mathrm{FNO},[\mathrm{M}+\mathrm{H}]^{+}$: 192.0631, found: 192.0579.

$\boldsymbol{O}$-(Benzyloxymethyl)hydroxylamine (2d): 2d was prepared through the procedure analogous to that of ${ }_{2 d}{ }^{-}{ }^{-}{ }^{\mathrm{NH}_{2}}$ 2b. Alkylation was performed using $N$-hydroxyphthalimide (322.6 mg. $2.0 \mathrm{mmol}$, 1.0 equiv), chloromethyl benzyl ether (439.1 mg, $2.8 \mathrm{mmol}, 1.4$ equiv), and $\mathrm{Et}_{3} \mathrm{~N}$ (286.0 mg, $2.8 \mathrm{mmol}, 1.4$ equiv) in $\mathrm{CH}_{2} \mathrm{Cl}_{2}$ (4.5 mL). Deprotection of phthalimide was conducted with $\mathrm{N}_{2} \mathrm{H}_{4} \cdot \mathrm{H}_{2} \mathrm{O}$ (150.2 mg, $3.0 \mathrm{mmol}$, 1.5 equiv) in $\mathrm{CH}_{3} \mathrm{Cl} / \mathrm{MeOH}=3: 1(5 \mathrm{~mL})$, which afforded $2 \mathbf{d}$ as colorless oil (213.4 mg, $1.4 \mathrm{mmol}$, 70\%).

IR (neat) $\tilde{v}: 2871 \mathrm{~cm}^{-1}$; ${ }^{1} \mathrm{H}$ NMR (500 MHz, $\left.\mathrm{CDCl}_{3}\right) \delta: 7.38-7.34$ (m, 4H), 7.32-7.29 (m, 1H), 5.51 (br s, 2H), 4.85 (s, 2H), 4.67 (s, 2H) ppm; ${ }^{13} \mathrm{C}$ NMR (125 MHz, $\mathrm{CDCl}_{3}$ ) $\delta: 137.1,128.4,127.7,98.5$, 69.9 ppm; HRMS (ESI) $m / z$ calcd. for $\mathrm{C}_{8} \mathrm{H}_{12} \mathrm{NO}_{2}$, [M] $]^{+}: 154.0865$, found: 154.0805 .

O-((2-Trimethylsilylethoxy)methyl)hydroxylamine (2e): 2e was prepared through the procedure $\mathrm{Me}_{3} \mathrm{Si} \sim ح_{\mathrm{O}} \mathrm{NH}_{2}$ analogous to that of $\mathbf{2 b}$. Alkylation was performed using $N$-hydroxyphthalimide $2 \mathrm{e}$ (815.5 mg. $5.0 \mathrm{mmol}, 1.0$ equiv), chloromethyl 2-(trimethylsilyl)ethyl ether (1.16 g, 7.0 mmol, 1.4 equiv), and $\mathrm{Et}_{3} \mathrm{~N}$ (708.3 mg, $7.0 \mathrm{mmol}, 1.4$ equiv) in $\mathrm{CH}_{2} \mathrm{Cl}_{2}$ (11.4 mL). Deprotection of phthalimide was conducted with $\mathrm{N}_{2} \mathrm{H}_{4} \cdot \mathrm{H}_{2} \mathrm{O}$ (375.5 mg, $7.5 \mathrm{mmol}, 1.5$ equiv) in $\mathrm{CH}_{3} \mathrm{Cl} / \mathrm{MeOH}=3: 1(10 \mathrm{~mL}$ ), which afforded 2e as colorless oil (550.5 mg, $3.1 \mathrm{mmol}$, 62\%). IR (neat) $\tilde{v}: 2953 \mathrm{~cm}^{-1}$; ${ }^{1} \mathrm{H} \mathrm{NMR}\left(500 \mathrm{MHz}, \mathrm{CDCl}_{3}\right) \delta: 5.47$ (br s, 2H), 4.73 (s, 2H), 3.64 (t, $J=8.0 \mathrm{~Hz}$, 2H), 0.96 (t, $J=8.0 \mathrm{~Hz}, 2 \mathrm{H}), 0.01$ (s, 9H) ppm; ${ }^{13} \mathrm{C}$ NMR (125 MHz, $\left.\mathrm{CDCl}_{3}\right) \delta: 98.8,65.7,18.3 \mathrm{ppm}$; HRMS (ESI) $\mathrm{m} / \mathrm{z}$ calcd. for $\mathrm{C}_{6} \mathrm{H}_{17} \mathrm{NO}_{2} \mathrm{Si},[\mathrm{M}+\mathrm{H}]^{+}$: 164.1107 , found: 164.1018 .

O-Benzoylhydroxylamine (S2a) ${ }^{7}$ : Colorless oil.

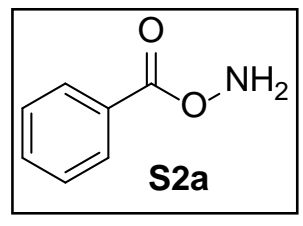

${ }^{1} \mathrm{H}$ NMR (400 MHz, CDCl $) \delta: 8.00(\mathrm{~d}, J=2.0 \mathrm{~Hz}, 2 \mathrm{H}), 7.58$ (t, $\left.J=7.6 \mathrm{~Hz}, 1 \mathrm{H}\right), 7.45$ (t, $J=7.6 \mathrm{~Hz}, 2 \mathrm{H}), 6.60$ (br s, 2H) ppm. 


\section{(C-3) General Procedure for Catalytic Aza-Michael Addition}
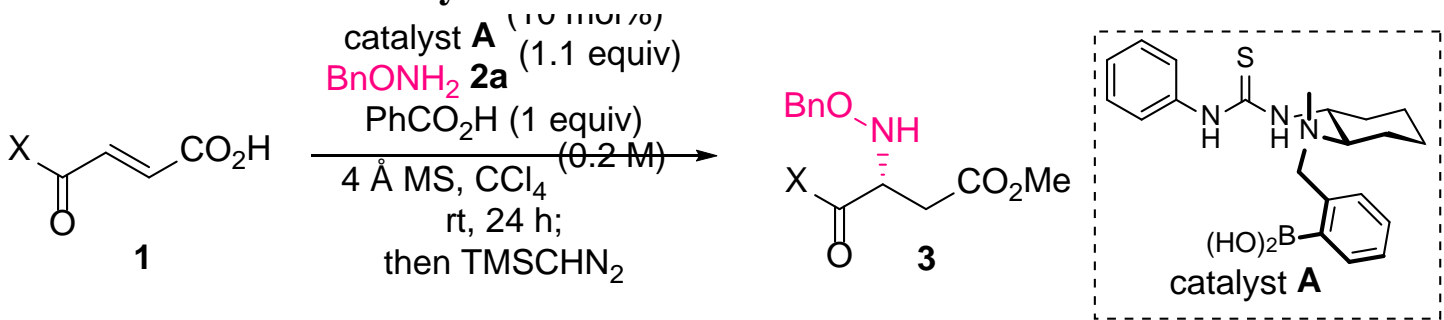

Prior to the reaction, $4 \AA$ MS was dried by heat-gun (>300 $\left.{ }^{\circ} \mathrm{C}, 15 \mathrm{~min}\right)$ under vacuum (ca. 2 Torr). To an oven-dried $10 \mathrm{~mL}$ screw tube were placed an organocatalyst (10 mol\%), substrate 1 (1.0 equiv), and benzoic acid (1.0 equiv), which were suspended in $\mathrm{CCl}_{4}(0.2 \mathrm{M})$ and sealed with a Teflon-coated screw cap. After stirring at room temperature for $10 \mathrm{~min}$, pre-heated $4 \AA \mathrm{MS}$ (500 mg/mmol) was added and the tube was capped and further stirred for 5 min. Hydroxylamine 2 (1.1 equiv) was then added and the system was closed again followed by stirring at ambient temperature for the indicated time. After the reaction progress was monitored by ${ }^{1} \mathrm{H}$ NMR analysis (a small amount of the mixture was transferred into an NMR tube). The reaction mixture was diluted in toluene/MeOH (3:1, $1 \mathrm{~mL})$ and treated with $\mathrm{TMSCHN}_{2}(10 \%$ in hexane, $1 \mathrm{~mL}$ ) and stirred for $30 \mathrm{~min}$. The excess $\mathrm{TMSCHN}_{2}$ was quenched with $\mathrm{AcOH}$, then the mixture was filtered through Celite ${ }^{\circledR}$ and the cake was washed with $\mathrm{MeOH}$. After the solvent was removed under reduced pressure, the residue was purified by silica-gel column chromatography (eluent: hexane/EtOAc, 1:1) to afford the product $\mathbf{3}$. The ee of $\mathbf{3}$ was estimated by chiral HPLC analysis.

1-tert-Butyl 4-methyl $N$-benzyloxy-D-aspartate (3aa): The reaction was carried out using 1a (34.4 mg,

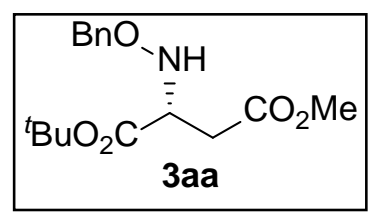
$200 \mu \mathrm{mol}, 1.0$ equiv) and 2a (27.0 mg, $220 \mu \mathrm{mol}, 1.1$ equiv) in the presence of catalyst A (7.9 mg, $20 \mu \mathrm{mol}, 10 \mathrm{~mol} \%$ ), benzoic acid (24.4 mg, $200 \mu \mathrm{mol}, 1.0$ equiv) and $4 \AA$ MS (100.0 mg) for $24 \mathrm{~h}$. The crude product was purified by silica-gel column chromatography (eluent: hexane/EtOAc, 3:1) afforded 3aa as colorless oil (40.5 mg, $164 \mu \mathrm{mol}, 80 \%, 93 \%$ ee).

$[\alpha]_{D}^{24}+5.4$ (c 1.00, $\left.\mathrm{CHCl}_{3}\right)$; IR (neat) $\tilde{v}: 3275,1731 \mathrm{~cm}^{-1} ;{ }^{1} \mathrm{H} \mathrm{NMR}\left(400 \mathrm{MHz}, \mathrm{CDCl}_{3}\right) \delta: 7.31$ (m, 5H), 6.18 (br s, 1H), 4.69 (s, 2H), 3.92 (br s, 1H), 3.68 (s, 3H), 2.76 (dd, $J=16.0,6.4$ Hz, 1H), 2.63 (dd, $J=$ 16.0, $6.4 \mathrm{~Hz}, 1 \mathrm{H}), 1.47$ (s, 9H) ppm; ${ }^{13} \mathrm{C} \mathrm{NMR}\left(125 \mathrm{MHz}, \mathrm{CDCl}_{3}\right) \delta: 171.3,170.6,137.5,128.3,128.2$ 127.7, 76.3, 64.2, 60.1, 51.8, 34.3, 27.9 ppm; HRMS (ESI) $\mathrm{m} / \mathrm{z}$ calcd. for $\mathrm{C}_{16} \mathrm{H}_{23} \mathrm{NO}_{5}$, [M+Na] $]^{+}$: 332.1468, found: 332.1470. The enantiomeric excess was estimated by chiral HPLC analysis (DAICEL CHIRALPAK IB, eluent: hexane/2-propanol = 99/1, flow rate: $1.0 \mathrm{~mL} / \mathrm{min}$. detector: $\mathrm{UV}$ at $220 \mathrm{~nm}$ ), $t_{\mathrm{R}}=12.0 \mathrm{~min}(\operatorname{minor})$, 10.5 min (major).

1-Benzyl 4-methyl $N$-benzyloxy-D-aspartate (3ba): The reaction was carried out using 1 b (82.4 mg, 400

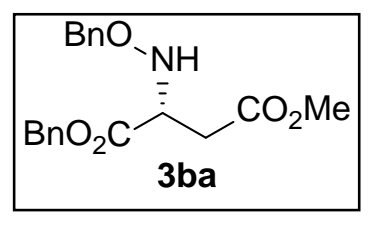
$\mu \mathrm{mol}, 1.0$ equiv) and 2a (54.2 mg, $440 \mu \mathrm{mol}, 1.1$ equiv) in the presence of catalyst A (15.9 mg, $40 \mu \mathrm{mol}, 10 \mathrm{~mol} \%)$, benzoic acid (48.8 mg, $400 \mu \mathrm{mol}, 1.0$ equiv) and 4 Å MS (200.0 mg) for $24 \mathrm{~h}$. The crude product was purified by silica-gel column chromatography (eluent: hexane/EtOAc, 2:1) afforded 3ba as colorless oil (69.6 mg, $201 \mu \mathrm{mol}, 50 \%$, 91\% ee).

$[\alpha]_{D}^{26}+4.2$ (c 0.94, $\mathrm{CHCl}_{3}$ ); IR (neat) $\tilde{v}: 1738 \mathrm{~cm}^{-1} ;{ }^{1} \mathrm{H}$ NMR (500 MHz, $\left.\mathrm{CDCl}_{3}\right) \delta: 7.34(\mathrm{~m}, 10 \mathrm{H}), 6.26$ (br s, 1H), 5.23 (d, $J=12.0 \mathrm{~Hz}, 1 \mathrm{H}), 5.19$ (d, $J=12.0 \mathrm{~Hz}, 1 \mathrm{H}), 4.71$ (d, $J=12.0 \mathrm{~Hz}, 1 \mathrm{H}$ ), 4.68 (d, $J=12.0$ 
Hz, 1H), 4.09 (t, $J=7.0 \mathrm{~Hz}, 1 \mathrm{H}$ ), 3.64 (s, 3H), 2.85 (dd, $J=16.0,6.0 \mathrm{~Hz}, 1 \mathrm{H}$ ), 2.70 (dd, $J=16.0,6.0 \mathrm{~Hz}$, 1H) ppm; ${ }^{13} \mathrm{C}$ NMR (125 MHz, $\left.\mathrm{CDCl}_{3}\right) \delta: 171.5,171.2,137.4,135.4,128.68,128.60,128.4,128.3,128.0$, 76.6, 67.2, 60.2, 52.0, 34.2 ppm; HRMS (ESI) m/z calcd. for $\mathrm{C}_{19} \mathrm{H}_{21} \mathrm{NO}_{5},[\mathrm{M}+\mathrm{Na}]^{+}$: 366.1312, found: 366.1290. The enantiomeric excess was estimated by chiral HPLC analysis (DAICEL CHIRALPAK IB, eluent: hexane/2-propanol $=98 / 2$, flow rate: $1.0 \mathrm{~mL} / \mathrm{min}$. detector: $\mathrm{UV}$ at $254 \mathrm{~nm}$ ), $t_{\mathrm{R}}=16.1 \mathrm{~min}$ (major), 17.4 min (minor).

1-Ethyl 4-methyl $N$-benzyloxy-D-aspartate (3ca): The reaction was carried out using 1c (57.6 mg, 400

$\mathrm{EtO}_{2} \overbrace{\mathbf{3} \text { ca }}^{\mathrm{BnO}_{3}^{\mathrm{NH}}} \mathrm{CO}_{2} \mathrm{Me}$
$\mu \mathrm{mol}, 1.0$ equiv) and 2a (54.2 mg, $440 \mu \mathrm{mol}, 1.1$ equiv) in the presence of catalyst A (15.9 mg, $40 \mu \mathrm{mol}, 10 \mathrm{~mol} \%$ ), benzoic acid (48.8 mg, $400 \mu \mathrm{mol}, 1.0$ equiv) and 4 Å MS (200.0 mg) for $24 \mathrm{~h}$. The crude product was purified by silica-gel column chromatography (eluent: hexane/EtOAc, 2:1) afforded 3ca as colorless oil (55.2 mg, $197.6 \mu \mathrm{mol}, 49 \%$, 94\% ee).

$[\alpha]_{D}^{26} 6.1$ (c 1.04, $\mathrm{CHCl}_{3}$ ); IR (neat) $\tilde{v}: 3265,1732 \mathrm{~cm}^{-1} ;{ }^{1} \mathrm{H} \mathrm{NMR}\left(400 \mathrm{MHz}, \mathrm{CDCl}_{3}\right) \delta: 7.31$ (m, 5H), 6.22 (br s, 1H), 4.69 (s, 2H), 4.23 (q, $J=6.8 \mathrm{~Hz}, 2 \mathrm{H}), 4.01$ (t, $J=6.4 \mathrm{~Hz}, 1 \mathrm{H}), 3.68$ (s, 3H), 2.81 (dd, $J=$ 16.4, $6.4 \mathrm{~Hz}, 1 \mathrm{H}), 1.28$ (t, $J=6.8 \mathrm{~Hz}, 3 \mathrm{H}) \mathrm{ppm} ;{ }^{13} \mathrm{C} \mathrm{NMR}\left(100 \mathrm{MHz}, \mathrm{CDCl}_{3}\right) \delta: 171.5,171.2,137.4,128.4$, 128.3, 127.8, 76.5, 61.4, 60.0, 51.9, 34.1, 14.0 ppm; HRMS (ESI) m/z calcd. for $\mathrm{C}_{14} \mathrm{H}_{19} \mathrm{NO}_{5}$, [M+Na] $]^{+}$ 304.1155, found: 304.1140. The enantiomeric excess was estimated by chiral HPLC analysis (DAICEL CHIRALPAK @, eluent: hexane/2-propanol =99/1, flow rate: $1.0 \mathrm{~mL} / \mathrm{min}$. detector: UV at $254 \mathrm{~nm}), t_{\mathrm{R}}=$ 16.9 min (major), $20.1 \mathrm{~min}$ (minor).

Methyl (R)-3-((benzyloxy)amino)-4-((S)-1-(tert-butoxy)-1-oxo-3-phenylpropan-2-yl)amino)-4-

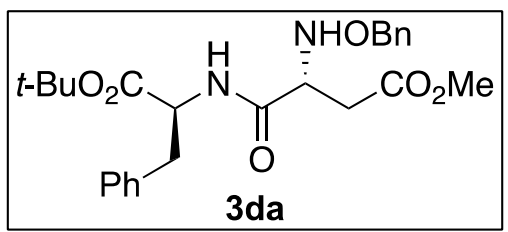
oxobutanoate (3da): The reaction was carried out using 1d (133.2 mg, $400 \mu \mathrm{mol}, 1.0$ equiv) and 2a (54.2 mg, $440 \mu \mathrm{mol}, 1.1$ equiv) in the presence of catalyst A (15.9 mg, $40 \mu \mathrm{mol}, 10 \mathrm{~mol} \%)$, benzoic acid (48.8 mg, $400 \mu \mathrm{mol}, 1.0$ equiv) and and $4 \AA$ MS (200.0 mg) for $24 \mathrm{~h}$. The crude product was purified by silica-gel column chromatography (eluent: hexane/EtOAc, 2:1) afforded 3da as colorless oil (120.0 mg, $263 \mu \mathrm{mol}, 66 \%, 67: 33 \mathrm{dr})$.

For major diastereomer: $[\alpha]_{D}^{21} 39.8$ (c 0.51, $\left.\mathrm{CHCl}_{3}\right)$; IR (neat) $\tilde{v}$ : 3381, 1730, $1675 \mathrm{~cm}^{-1}$; ${ }^{1} \mathrm{H}$ NMR (500 $\left.\mathrm{MHz}, \mathrm{CDCl}_{3}\right) \delta: 7.34-7.14(\mathrm{~m}, 10 \mathrm{H}), 6.19(\mathrm{~d}, J=5.5 \mathrm{~Hz}, 1 \mathrm{H}), 4.73(\mathrm{dt}, J=7.5,6.0 \mathrm{~Hz}, 1 \mathrm{H}), 4.68(\mathrm{~d}, J=$ $12.0 \mathrm{~Hz}, 1 \mathrm{H}$ ), 4.61 (d, $J=12.0 \mathrm{~Hz}, 1 \mathrm{H}), 3.81$ (m, 1H), 3.66 (s, 3H), 3.11 (dd, $J=15.0,6.0 \mathrm{~Hz}, 1 \mathrm{H}$ ), 3.07 (dd, $J=15.0,6.0 \mathrm{~Hz}, 1 \mathrm{H}$ ), 2.87 (dd, $J=17.5,9.0 \mathrm{~Hz}, 1 \mathrm{H}$ ), 2.77 (dd, $J=17.0,9.0 \mathrm{~Hz}, 1 \mathrm{H}$ ), 1.40 (s, 9H) ppm; ${ }^{13} \mathrm{C}$ NMR (125 MHz, $\left.\mathrm{CDCl}_{3}\right) \delta: 172.5,170.4,170.2$, 137.2, 136.3, 129.6, 128.57, 128.52, 128.4, 128.1, 127.0, 82.4, 76.3, 60.5, 53.6, 52.0, 38.2, 32.2, 28.0 ppm; HRMS (ESI) $m / z$ calcd. for $\mathrm{C}_{25} \mathrm{H}_{32} \mathrm{~N}_{2} \mathrm{O}_{6},[\mathrm{M}+\mathrm{H}]^{+}$: 457.2333, found: 457.2353 .

For minor diastereomer: $[\alpha]_{D}^{21} 19.7$ (c 0.60, $\mathrm{CHCl}_{3}$ ); IR (neat) $\tilde{v}$ : 3388, 1732, $1674 \mathrm{~cm}^{-1}$; ${ }^{1} \mathrm{H}$ NMR (500 $\left.\mathrm{MHz}, \mathrm{CDCl}_{3}\right) \delta: 7.33-7.17(\mathrm{~m}, 10 \mathrm{H}), 6.18(\mathrm{~d}, J=6.0 \mathrm{~Hz}, 1 \mathrm{H}), 4.73(\mathrm{dt}, J=7.5,6.0 \mathrm{~Hz}, 1 \mathrm{H}), 4.62(\mathrm{~d}, J=$ $11.5 \mathrm{~Hz}, 1 \mathrm{H}$ ), 4.59 (d, $J=11.5 \mathrm{~Hz}, 1 \mathrm{H}$ ), 3.82 (ddd, $J=8.0,7.0,4.5 \mathrm{~Hz}, 1 \mathrm{H}$ ), 3.65 (s, 3H), 3.11 (dd, $J=14.0$, $6.5 \mathrm{~Hz}, 1 \mathrm{H}$ ), 3.08 (dd, $J=14.0,6.0 \mathrm{~Hz}, 1 \mathrm{H}$ ), 2.83 (dd, $J=12.0,4.5 \mathrm{~Hz}, 1 \mathrm{H}$ ), 2.71 (dd, $J=12.0,8.0 \mathrm{~Hz}$, 1H), 1.44 (s, 9H) ppm; ${ }^{13} \mathrm{C}$ NMR (125 MHz, $\left.\mathrm{CDCl}_{3}\right) \delta: 172.3,170.47,170.42,137.1,136.2,129.7,128.4$, 128.1, 127.0, 82.4, 76.2, 60.6, 53.6, 52.0, 38.1, 32.5, 28.0 ppm; HRMS (ESI) $\mathrm{m} / \mathrm{z}$ calcd. for $\mathrm{C}_{25} \mathrm{H}_{32} \mathrm{~N}_{2} \mathrm{O}_{6}$, 
$[\mathrm{M}+\mathrm{H}]^{+}:$457.2333, found: 457.2354.

Methyl (R)-3-((benzyloxy)amino)-4-((R)-1-(tert-butoxy)-1-oxo-3-phenylpropan-2-yl)amino)-4$t-\mathrm{BuO}_{2} \mathrm{C}_{3}$ oxobutanoate (3ea): The reaction was carried out using 1e (133.2 mg, $400 \mu \mathrm{mol}, 1.0$ equiv) and 2a (54.2 mg, $440 \mu \mathrm{mol}, 1.1$ equiv) in the presence of catalyst A (15.9 mg, $40 \mu \mathrm{mol}, 10 \mathrm{~mol} \%)$, benzoic acid (48.8 mg, $400 \mu \mathrm{mol}, 1.0$ equiv) and $4 \AA$ MS (200.0 mg) for $24 \mathrm{~h}$. The crude product was purified by silica-gel column chromatography (eluent: hexane/EtOAc, 2:1) afforded 3ea as colorless oil (128.2 mg, $280 \mu \mathrm{mol}, 70 \%, 75: 25 \mathrm{dr})$.

For major diastereomer: $[\alpha]_{D}^{21}-15.0$ (c 0.66, $\left.\mathrm{CHCl}_{3}\right)$; IR (neat) $\tilde{v}: 3381,1731,1674 \mathrm{~cm}^{-1}$; ${ }^{1} \mathrm{H} \mathrm{NMR}(500$ $\left.\mathrm{MHz}, \mathrm{CDCl}_{3}\right) \delta$ : 7.34-7.16 (m, 10H), 6.19 (br s, $\left.1 \mathrm{H}\right), 4.73$ (dt, $\left.J=7.5,6.0 \mathrm{~Hz}, 1 \mathrm{H}\right), 4.61$ (d, $J=11.5 \mathrm{~Hz}$, $1 \mathrm{H}), 4.59$ (d, $J=11.5 \mathrm{~Hz}, 1 \mathrm{H}), 3.82$ (m, 1H), 3.66 (s, 3H), 3.11 (dd, $J=14.0,6.5 \mathrm{~Hz}, 1 \mathrm{H}), 3.08$ (dd, $J=$ 14.0, $6.5 \mathrm{~Hz}, 1 \mathrm{H}$ ), 2.83 (dd, $J=12.0,4.5 \mathrm{~Hz}, 1 \mathrm{H}$ ), 2.71 (dd, $J=17.0,8.0 \mathrm{~Hz}, 1 \mathrm{H}$ ), 1.41 (s, 9H) ppm; ${ }^{13} \mathrm{C}$ NMR (125 MHz, $\left.\mathrm{CDCl}_{3}\right) \delta:$ 172.3, 170.47, 170.42, 137.1, 136.2, 129.7, 128.5, 128.4, 128.1, 127.0, 82.4, 76.2, 60.6, 53.6, 52.0, 38.1, 32.5, 28.0 ppm; HRMS (ESI) $\mathrm{m} / \mathrm{z}$ calcd. for $\mathrm{C}_{25} \mathrm{H}_{32} \mathrm{~N}_{2} \mathrm{O}_{6},[\mathrm{M}+\mathrm{H}]^{+}$: 457.2333, found: 457.2328.

For minor diastereomer: $\quad[\alpha]_{D}^{22}-43.9\left(c\right.$ 0.57, $\left.\mathrm{CHCl}_{3}\right)$; IR (neat) $\tilde{v}$ : 3383, 1729, $1674 \mathrm{~cm}^{-1}$; ${ }^{1} \mathrm{H} \mathrm{NMR}$ $\left(500 \mathrm{MHz}, \mathrm{CDCl}_{3}\right) \delta: 7.32-7.14(\mathrm{~m}, 10 \mathrm{H}), 6.19(\mathrm{~d}, J=6.0 \mathrm{~Hz}, 1 \mathrm{H}), 4.74(\mathrm{dt}, J=7.5,6.5 \mathrm{~Hz}, 1 \mathrm{H}), 4.65(\mathrm{~d}$, $J=11.5 \mathrm{~Hz}, 1 \mathrm{H}), 4.59$ (d, $J=11.5 \mathrm{~Hz}, 1 \mathrm{H}), 3.81$ (m, 1H), 3.66 (s, 3H), 3.12 (dd, $J=14.0,6.0 \mathrm{~Hz}, 1 \mathrm{H})$, 3.07 (dd, $J=14.0,6.0 \mathrm{~Hz}, 1 \mathrm{H}$ ), 2.87 (dd, $J=17.0,5.0 \mathrm{~Hz}, 1 \mathrm{H}$ ), 2.77 (dd, $J=17.5,8.0 \mathrm{~Hz}, 1 \mathrm{H}$ ), 1.40 (s, 9H) ppm; ${ }^{13} \mathrm{C}$ NMR (125 MHz, $\left.\mathrm{CDCl}_{3}\right) \delta: 172.5,170.4,170.2,137.1,136.3,129.5,128.5,128.49,128.46$, 128.0, 127.0, 82.3, 76.2, 60.4, 53.6, 51.9, 38.1, 32.2, 28.0 ppm; HRMS (ESI) $\mathrm{m} / \mathrm{z}$ calcd. for $\mathrm{C}_{25} \mathrm{H}_{32} \mathrm{~N}_{2} \mathrm{O}_{6}$, $[\mathrm{M}+\mathrm{H}]^{+}:$457.2333, found: 457.2355 .

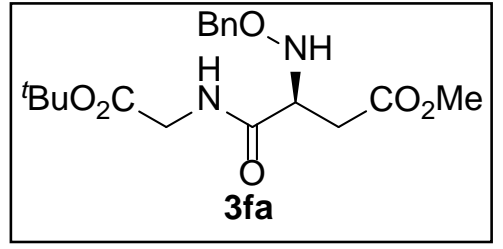

\section{Methyl}

(S)-3-((benzyloxy)amino)-4-((tert-

butoxycarbonylmethyl)amino)-4-oxobutanoate (3fa): The reaction was carried out using 1f (91.6 mg, $400 \mu \mathrm{mol}, 1.0$ equiv) and 2a (54.2 mg, 440 $\mu \mathrm{mol}, 1.1$ equiv) in the presence of catalyst ent-A (15.9 mg, $40 \mu \mathrm{mol}, 10$ mol\%), benzoic acid (48.8 mg, $400 \mu \mathrm{mol}, 1.0$ equiv) and $4 \AA \mathrm{MS}$ (200.0 mg) for $24 \mathrm{~h}$. The crude product was purified by silica-gel column chromatography (eluent: hexane/EtOAc, 2:1) afforded 3fa as colorless oil (241.4 mg, $266 \mu \mathrm{mol}, 66 \%, 63 \%$ ee).

$[\alpha]_{D}^{24} 4.6$ (c 1.46, $\mathrm{CHCl}_{3}$ ); IR (neat) $\tilde{v}: 1733,1669 \mathrm{~cm}^{-1} ;{ }^{1} \mathrm{H}$ NMR (500 MHz, $\left.\mathrm{CDCl}_{3}\right) \delta: 7.34-7.28$ (m, 4H), 7.19 (m, 1H), 6.46 (br s, 1H), 4.73 (s, 2H), 4.03 (d, $J=5.5 \mathrm{~Hz}, 1 \mathrm{H}$ ), 3.88 (dd, $J=5.0,2.0 \mathrm{~Hz}, 1 \mathrm{H}$ ), 3.85 (dd, $J=5.0,2.0 \mathrm{~Hz}, 1 \mathrm{H}$ ), 3.66 (s, 3H), 2.89 (dd, $J=17.0,8.0 \mathrm{~Hz}$ ), 2.78 (dd, $J=17.0,8.0 \mathrm{~Hz}), 1.46$ (s, 9H) ppm; ${ }^{13} \mathrm{C}$ NMR (125 MHz, $\left.\mathrm{CDCl}_{3}\right) \delta: 172.5,170.8,168.7,130.6,128.7,128.5,128.1,82.3,76.2,60.4,52.0$, 42.0, 32.3, 28.1 ppm; HRMS (ESI) $\mathrm{m} / \mathrm{z}$ calcd. for $\mathrm{C}_{18} \mathrm{H}_{26} \mathrm{~N}_{2} \mathrm{O}_{6},[\mathrm{M}+\mathrm{H}]^{+}: 367.1864$, found: 367.1789. The enantiomeric excess was estimated by chiral HPLC analysis (DAICEL CHIRALPAK IB, eluent: hexane/2propanol $=96 / 4$, flow rate: $1.0 \mathrm{~mL} / \mathrm{min}$. detector: $\mathrm{UV}$ at $254 \mathrm{~nm}$ ), $t_{\mathrm{R}}=29.6 \mathrm{~min}$ (major), $27.7 \mathrm{~min}$ (minor). 


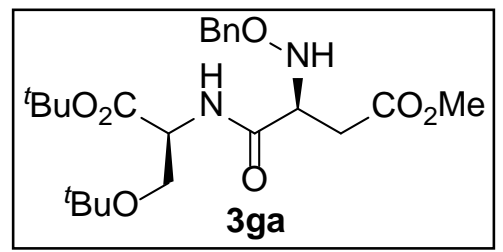

oxobutanoate (3ga): The reaction was carried out using 1 g (126 mg, 400 $\mu \mathrm{mol}, 1.0$ equiv) and $2 \mathbf{a}(54.2 \mathrm{mg}, 440 \mu \mathrm{mol}, 1.1$ equiv) in the presence of catalyst ent-A (15.9 mg, $40 \mu \mathrm{mol}, 10 \mathrm{~mol} \%$ ), benzoic acid (48.8 mg, 400 $\mu \mathrm{mol}, 1.0$ equiv) and $4 \AA$ MS (200.0 mg) for $24 \mathrm{~h}$. The crude product was purified by silica-gel column chromatography (eluent: hexane/EtOAc, 2:1) afforded 3da as colorless oil (366.6 mg, $322 \mu \mathrm{mol}, 81 \%, 73: 27 \mathrm{dr}$ ).

For major diastereomer: $[\alpha]_{D}^{21} 33.2$ (c 1.02, $\mathrm{CHCl}_{3}$ ); IR (neat) $\tilde{v}$ : 3403, 1735, $1679 \mathrm{~cm}^{-1}$; ${ }^{1} \mathrm{H}$ NMR (500 $\mathrm{MHz}_{\mathrm{CDCl}}$ ) $\delta: 7.66(\mathrm{~d}, J=8.0 \mathrm{~Hz}, 1 \mathrm{H}), 7.37-7.31(\mathrm{~m}, 5 \mathrm{H}), 6.26(\mathrm{~d}, J=10.0 \mathrm{~Hz}, 1 \mathrm{H}), 4.80(\mathrm{dd}, J=15.0$, $11.5 \mathrm{~Hz}, 2 \mathrm{H}), 4.60$ (dt, $J=9.0,2.5 \mathrm{~Hz}, 1 \mathrm{H}), 3.90$ (dt, $J=7.5,4.5 \mathrm{~Hz}, 1 \mathrm{H}$ ), 3.81 (dd, $J=17.0,4.5 \mathrm{~Hz}, 1 \mathrm{H}$ ), 3.67 (s, 3H), 3.54 (dd, $J=8.5,3.0 \mathrm{~Hz}, 1 \mathrm{H}), 2.91$ (dd, $J=17.0,4.5 \mathrm{~Hz}, 1 \mathrm{H}$ ), 2.76 (dd, $J=17.0,9.0 \mathrm{~Hz}, 1 \mathrm{H}$ ), 1.47 (s, 9H), 1.13 (s, 9H) ppm; ${ }^{13} \mathrm{C}$ NMR (125 MHz, $\left.\mathrm{CDCl}_{3}\right) \delta: 172.4,170.5,169.2,137.3,128.6,128.5$, 128.0, 81.8, 76.4, 73.1, 60.7, 53.2, 52.0, 32.6, 28.1, 27.4 ppm; HRMS (ESI) m/z calcd. for $\mathrm{C}_{25} \mathrm{H}_{32} \mathrm{~N}_{2} \mathrm{O}_{6}$, $[\mathrm{M}+\mathrm{H}]^{+}:$453.2595, found: 453.2570 .

For minor diastereomer: $[\alpha]_{D}^{21} 1.0$ (c 0.96, $\mathrm{CHCl}_{3}$ ); IR (neat) $\tilde{v}: 3405,1737,1679 \mathrm{~cm}^{-1}$; ${ }^{1} \mathrm{H}$ NMR (500 $\left.\mathrm{MHz}, \mathrm{CDCl}_{3}\right) \delta: 7.61(\mathrm{~d}, J=8.5 \mathrm{~Hz}, 1 \mathrm{H}), 7.34-7.26$ (m, 5H), 6.26 (d, $\left.J=6.5 \mathrm{~Hz}, 1 \mathrm{H}\right), 4.78(\mathrm{dd}, J=17.0$, $11.0 \mathrm{~Hz}, 2 \mathrm{H}$ ), 4.61 (dt, $J=8.5,2.5 \mathrm{~Hz}, 1 \mathrm{H}$ ), 3.91 (ddd, $J=9.0,6.5,3.0 \mathrm{~Hz}, 1 \mathrm{H}$ ), 3.81 (dd, $J=9.0,3.0 \mathrm{~Hz}$, 1H), 3.67 (s, 3H), 3.54 (dd, $J=9.0,3.0 \mathrm{~Hz}, 1 \mathrm{H}$ ), 2.93 (dd, $J=17.5,5.0 \mathrm{~Hz}, 1 \mathrm{H}$ ), 2.80 (dd, $J=17.5,9.0 \mathrm{~Hz}$, 1H), 1.47 (s, 9H), 1.12 (s, 9H) ppm; ${ }^{13} \mathrm{C}$ NMR (125 MHz, $\left.\mathrm{CDCl}_{3}\right) \delta: 172.4,170.5,169.3,137.3,128.58$, 128.51, 128.0, 81.9, 76.4, 73.1, 62.2, 60.5, 53.2, 51.9, 32.3, 28.1, 27.4 ppm; HRMS (ESI) m/z calcd. for $\mathrm{C}_{25} \mathrm{H}_{32} \mathrm{~N}_{2} \mathrm{O}_{6},[\mathrm{M}+\mathrm{H}]^{+}:$453.2595, found: 453.2524 .

1-tert-Butyl 4-methyl $N$-(4-methoxybenzyl)oxy-D-aspartate (3ab): The reaction was carried out using

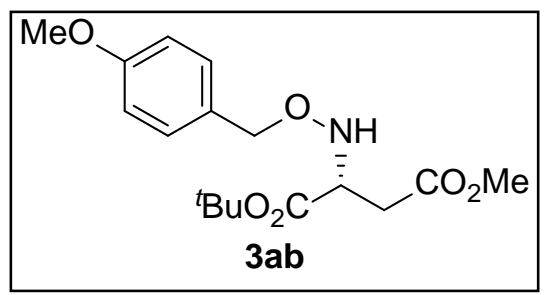
1a (68.8 mg, 400 mol, 1.0 equiv) and $\mathbf{2 b}$ (67.4 mg, $440 \mu \mathrm{mol}, 1.1$ equiv) in the presence of catalyst $\mathbf{A}(15.9 \mathrm{mg}, 40 \mu \mathrm{mol}, 10 \mathrm{~mol} \%)$, benzoic acid (48.8 mg, $400 \mu \mathrm{mol}, 1.0$ equiv) and $4 \AA$ MS (200.0 mg) for $24 \mathrm{~h}$. The crude product was purified by silica-gel column chromatography (eluent: hexane/EtOAc, 2:1) afforded 3ab as colorless oil (101.8 mg, $300 \mu \mathrm{mol}$,

$75 \%, 89 \%$ eе).

$[\alpha]_{D}^{23} 8.2$ (c 0.68, $\mathrm{CHCl}_{3}$ ); IR (neat) $\tilde{v}: 1732 \mathrm{~cm}^{-1} ;{ }^{1} \mathrm{H}$ NMR $\left(500 \mathrm{MHz}, \mathrm{CDCl}_{3}\right) \delta: 7.26$ (t, $\left.J=7.0 \mathrm{~Hz}, 2 \mathrm{H}\right)$, 6.86 (d, $J=7.0 \mathrm{~Hz}, 2 \mathrm{H}$ ), 6.12 (br s, 1H), 4.61 (s, 2H), 3.80 (s, 3H), 3.68 (s, 3H), 2.75 (dd, J = 16.0, 7.5 Hz, 1H), 2.62 (dd, $J=16.0,7.5 \mathrm{~Hz}, 1 \mathrm{H}), 1.46$ (s, 9H) ppm; ${ }^{13} \mathrm{C}$ NMR (125 MHz, $\left.\mathrm{CDCl}_{3}\right) \delta: 171.4,170.7,159.4$, 130.1, 129.7, 113.7, 82.1, 76.1, 60.7, 55.3, 51.9, 34.4, 28.0 ppm; HRMS (ESI) m/z calcd. for $\mathrm{C}_{17} \mathrm{H}_{25} \mathrm{NO}_{6}$, $[\mathrm{M}+\mathrm{H}]^{+}:$340.1755, found: 340.1744. The enantiomeric excess was estimated by chiral HPLC analysis (DAICEL CHIRALPAK IB, eluent: hexane/2-propanol = 98/2, flow rate: $1.0 \mathrm{~mL} / \mathrm{min}$. detector: $\mathrm{UV}$ at 254 $\mathrm{nm}), t_{\mathrm{R}}=13.3 \mathrm{~min}$ (major), $14.1 \mathrm{~min}$ (minor). 
1-tert-Butyl 4-methyl $N$-(4-trifluoromethyl)benzyloxy-D-aspartate (3ac): The reaction was carried out

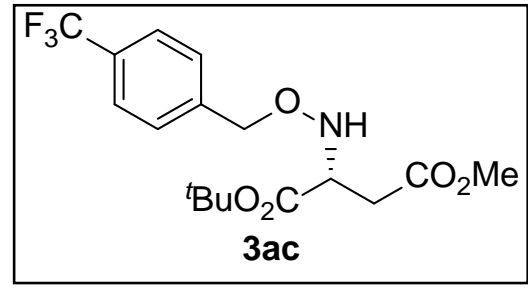
using 1a (34.4 mg, $200 \mu \mathrm{mol}, 1.0$ equiv) and 2c (42.0 mg, $220 \mu \mathrm{mol}, 1.1$ equiv) in the presence of catalyst $\mathbf{A}$ (7.9 $\mathrm{mg}, 20 \mu \mathrm{mol}, 10 \mathrm{~mol} \%$ ) and benzoic acid (48.8 mg, $400 \mu \mathrm{mol}, 1.0$ equiv) and $4 \AA \mathrm{MS}$ (200.0 mg) for 24 h. The crude product was purified by silica-gel column chromatography (eluent: hexane/EtOAc, 3:1) afforded 3ac as colorless oil

(48.2 mg, $128 \mu \mathrm{mol}, 64 \%$, 96\% ee).

$[\alpha]_{D}^{25} 6.6$ (c 1.90, $\mathrm{CHCl}_{3}$ ); IR (neat) $\tilde{v}: 1732,1324 \mathrm{~cm}^{-1} ;{ }^{1} \mathrm{H} \mathrm{NMR}\left(400 \mathrm{MHz}, \mathrm{CDCl}_{3}\right) \delta: 7.60$ (d, $J=8.0$ Hz, 1H), 7.44 (d, $J=8.0 \mathrm{~Hz}, 1 \mathrm{H}), 6.25$ (d, $J=6.0 \mathrm{~Hz}, 1 \mathrm{H}), 4.75$ (s, 2H), 3.93 (dt, $J=6.0 \mathrm{~Hz}, 1 \mathrm{H}$ ), 3.67 (s, 3H), 2.75 (dd, $J=16.0,6.5 \mathrm{~Hz}, 1 \mathrm{H}), 2.61$ (dd, $J=16.0,6.5 \mathrm{~Hz}, 1 \mathrm{H}), 1.48$ (s, 9H) ppm; ${ }^{13} \mathrm{C}$ NMR $(125$ $\left.\mathrm{MHz}, \mathrm{CDCl}_{3}\right) \delta: 171.1,170.5,141.7,129.9(\mathrm{q}, J=32.6 \mathrm{~Hz}), 128.2,125.2(\mathrm{q}, J=3.7 \mathrm{~Hz}), 124.1(\mathrm{q}, J=270$ $\mathrm{Hz}$ ), 82.2, 75.4, 60.7, 51.8, 51.7, 34.2, 27.9 ppm; HRMS (ESI) $\mathrm{m} / \mathrm{z}$ calcd. for $\mathrm{C}_{17} \mathrm{H}_{22} \mathrm{NO}_{5} \mathrm{~F}_{3}$, [M+Na] 400.1342, found: 400.1333. The enantiomeric excess was estimated by chiral HPLC analysis (DAICEL CHIRALPAK IB, eluent: hexane/2-propanol = 99/1, flow rate: $1.0 \mathrm{~mL} / \mathrm{min}$. detector: $\mathrm{UV}$ at $254 \mathrm{~nm}), t_{\mathrm{R}}=$ 15.8 min (minor), 20.2 min (major).

1-tert-Butyl 4-methyl $N$-(benzyloxy)methoxy-D-aspartate (3ad): The reaction was carried out using 1a

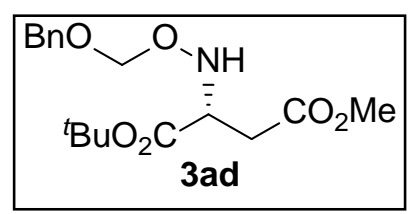
(34.4 mg, $200 \mu \mathrm{mol}, 1.0$ equiv) and 2d (36.7 mg, $220 \mu \mathrm{mol}, 1.1$ equiv) in the presence of catalyst A (7.9 mg, $20 \mu \mathrm{mol}, 10 \mathrm{~mol} \%)$ and benzoic acid (48.8 mg, $400 \mu \mathrm{mol}, 1.0$ equiv) and $4 \AA$ MS (200.0 mg) for $24 \mathrm{~h}$. The crude product was purified by silica-gel column chromatography (eluent: hexane/EtOAc, 3:1) afforded 3ad as colorless oil (40.0 mg, $122 \mu \mathrm{mol}, 60 \%, 87 \%$ ee).

$[\alpha]_{D}^{25} 4.4$ (c 1.06, $\mathrm{CHCl}_{3}$ ); IR (neat) $\tilde{v}: 1731 \mathrm{~cm}^{-1} ;{ }^{1} \mathrm{H}$ NMR (400 MHz, $\left.\mathrm{CDCl}_{3}\right) \delta: 7.31$ (m, 5H), 6.43 (d, $J=6.4 \mathrm{~Hz}, 1 \mathrm{H}$ ), 4.86 (s, 2H), 4.65 (s, 2H), 3.98 (dt, $J=10.0,6.4 \mathrm{~Hz}, 1 \mathrm{H}$ ), 3.69 (s, 3H), 2.77 (dd, $J=16.4$, $6.4 \mathrm{~Hz}, 1 \mathrm{H}), 2.67$ (dd, $J=16.4,6.4 \mathrm{~Hz}, 1 \mathrm{H}), 1.48$ (s, 9H) ppm; ${ }^{13} \mathrm{C}$ NMR $\left(100 \mathrm{MHz}, \mathrm{CDCl}_{3}\right) \delta: 171.2$, 170.6, 137.7, 128.4, 127.79, 127.67, 82.1, 69.9, 60.8, 51.8, 34.4, 27.9 ppm; HRMS (ESI) m/z calcd. for $\mathrm{C}_{17} \mathrm{H}_{25} \mathrm{NO}_{6},[\mathrm{M}+\mathrm{Na}]^{+}:$362.1574, found: 362.1588. The enantiomeric excess was estimated by chiral HPLC analysis (DAICEL CHIRALPAK IB, eluent: hexane/2-propanol = 98/2, flow rate: $1.0 \mathrm{~mL} / \mathrm{min}$. detector: $\mathrm{UV}$ at $220 \mathrm{~nm}$ ), $t_{\mathrm{R}}=16.1 \mathrm{~min}$ (major), $17.4 \mathrm{~min}$ (minor).

1-tert-Butyl 4-methyl $N$-(2-trimethylsilylethoxy)methoxy-D-aspartate (3ae): The reaction was carried

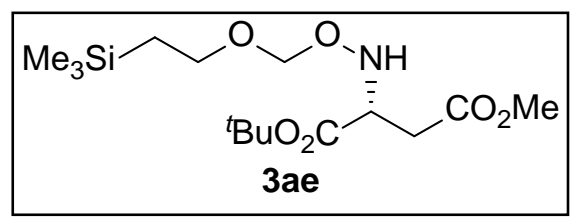
out using 1a (34.4 mg, $200 \mu \mathrm{mol}, 1.0$ equiv) and 2e (33.6 mg, $220 \mu \mathrm{mol}$, 1.1 equiv) in the presence of catalyst $\mathbf{A}$ (7.9 mg, $20 \mu \mathrm{mol}, 10 \mathrm{~mol} \%)$, benzoic acid (48.8 mg, $400 \mu \mathrm{mol}, 1.0$ equiv) and $4 \AA$ MS (200.0 mg) for $24 \mathrm{~h}$. The crude product was purified by silica-gel column chromatography (eluent: hexane/EtOAc, 3:1) afforded 3ae as colorless oil (48.9 mg, $142 \mu \mathrm{mol}, 70 \%$ ). Since 3ae was not detectable on HPLC, $N$-benzoylation was conducted to estimate the ee of $3 \mathbf{a e}$.

$[\alpha]_{D}^{25}+4.3$ (c 1.00, $\mathrm{CHCl}_{3}$ ); IR (neat) $\tilde{v}: 1738 \mathrm{~cm}^{-1} ;{ }^{1} \mathrm{H} \mathrm{NMR}\left(400 \mathrm{MHz}, \mathrm{CDCl}_{3}\right) \delta: 6.36$ (d, $J=6.4 \mathrm{~Hz}$, 1H), 4.75 (s, 2H), 3.94 (dt, $J=12.8 \mathrm{~Hz}, 6.4 \mathrm{~Hz}$ ), 3.68 (s, 3H), 3.62 (t, $J=8.4 \mathrm{~Hz}, 2 \mathrm{H}), 1.45$ (s, $9 \mathrm{H}), 0.94$ (t, $J=8.4 \mathrm{~Hz}, 2 \mathrm{H}) \mathrm{ppm} ;{ }^{13} \mathrm{C} \mathrm{NMR}\left(125 \mathrm{MHz}, \mathrm{CDCl}_{3}\right) \delta: 171.2,170.6,97.7,82.0,65.7,60.8,51.8,34.4,27.9$, 18.1 ppm; HRMS (ESI) m/z calcd. for $\mathrm{C}_{15} \mathrm{H}_{31} \mathrm{NO}_{6} \mathrm{Si}$, [M+Na] $]^{+}: 372.1813$, found: 372.1820. 
1-tert-Butyl 4-methyl $N$-benzoyl- $N$-(2-trimethylsilylethoxy)methoxy-D-aspartate (3ae'): To a solution

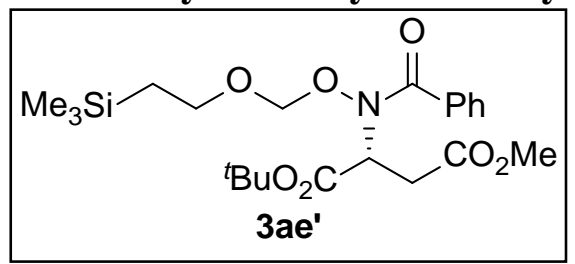

of 3ae $(34.9 \mathrm{mg}, 100 \mu \mathrm{mol}, 1.0$ equiv) in EtOAc $(1 \mathrm{~mL})$ were added $\mathrm{BzCl}\left(21.1 \mathrm{mg}, 150 \mu \mathrm{mol}, 1.5\right.$ equiv) and sat. $\mathrm{NaHCO}_{3}$ aq. $(1 \mathrm{~mL})$ at 0 ${ }^{\circ} \mathrm{C}$. The mixture was allowed to warm to room temperature and stirred for $3 \mathrm{~h}$. Added brine (2 mL) and extracted with EtOAc (5 mL, 2 times).

The crude product was purified by silica-gel column chromatography (eluent: hexane/EtOAc, 5:1) afforded 3ae' as colorless oil (38.8 mg, $85 \mu \mathrm{mol}, 85 \%$ ).

$[\alpha]_{D}^{25} 0.71$ (c 5.84, $\mathrm{CHCl}_{3}$ ); IR (neat) $\tilde{v}: 1736,1648 \mathrm{~cm}^{-1} ;{ }^{1} \mathrm{H}$ NMR (500 MHz, $\left.\mathrm{CDCl}_{3}\right) \delta: 7.67$ (d, $J=7.0$ Hz, 2H), 7.46 (t, $J=7.0$ Hz, 1H), 7.41 (t, $J=7.0$ Hz, 2H) 5.06 (br, 1H), 4.85 (br, 2H), 3.73 (s, 3H), 3.49 (s, 2H), 3.21 (dd, $J=17.0,7.0 \mathrm{~Hz}, 1 \mathrm{H}$ ), 2.97 (dd, $J=17.0,7.0 \mathrm{~Hz}, 1 \mathrm{H}$ ), 1.48 (s, 9H), 0.85 (t, $J=8.0 \mathrm{~Hz}$, 2H), 0.018 (s, 9H) ppm; ${ }^{13} \mathrm{C}$ NMR (125 MHz, $\left.\mathrm{CDCl}_{3}\right) \delta:$ 198.7, 171.3, 167.6, 134.2, 130.9, 128.3, 128.2, 99.6, 82.9, 67.7, 61.1, 52.0, 34.0, 27.9, 17.9 ppm; HRMS (ESI) $\mathrm{m} / \mathrm{z}$ calcd. for $\mathrm{C}_{22} \mathrm{H}_{35} \mathrm{NO}_{7} \mathrm{Si}$, $[\mathrm{M}+\mathrm{H}]^{+}$: 453.2111, found: 453.2183. The enantiomeric excess was estimated by chiral HPLC analysis (DAICEL CHIRALPAK IB, eluent: hexane $/ 2$-propanol = 1, flow rate: $1.0 \mathrm{~mL} / \mathrm{min}$. detector: $\mathrm{UV}$ at $254 \mathrm{~nm}), t_{\mathrm{R}}=18.9$ min (major), 23.7 min (minor).

1-Benzyl 4-methyl $N$-(2-trimethylsilylethoxy)methoxy-L-aspartate (3be): The reaction was carried out

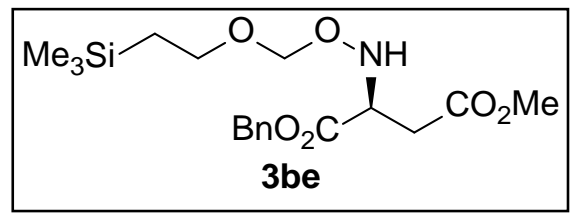
using $1 \mathbf{b}$ (82.5 mg, $400 \mu \mathrm{mol}, 1.0$ equiv) and $2 \mathbf{e}$ (67.3 $\mathrm{mg}, 440 \mu \mathrm{mol}$, 1.1 equiv) in the presence of catalyst ent-A (15.9 mg, $40 \mu \mathrm{mol}, 10$ mol\%), benzoic acid (48.8 mg, $400 \mu \mathrm{mol}, 1.0$ equiv) and $4 \AA$ MS (200.0 $\mathrm{mg}$ ) for $24 \mathrm{~h}$. The crude product was purified by silica-gel column chromatography (eluent: hexane/EtOAc, 3:1) afforded 3be as colorless oil (268.4 mg, $284 \mu \mathrm{mol}, 70 \%, 86 \%$ ee).

$[\alpha]_{D}^{26}+4.7$ (c 1.00, $\mathrm{CHCl}_{3}$ ); IR (neat) $\tilde{v}: 1739 \mathrm{~cm}^{-1} ;{ }^{1} \mathrm{H}$ NMR (500 MHz, $\left.\mathrm{CDCl}_{3}\right) \delta: 7.35$ (m, $\left.5 \mathrm{H}\right), 6.42$ (d $J=7.0 \mathrm{~Hz}, 1 \mathrm{H}), 5.21$ (s, 2H), $4.76(\mathrm{~s}, 2 \mathrm{H}), 4.12$ (dt, $J=7.0,6.0 \mathrm{~Hz}, 1 \mathrm{H}), 3.65$ (s, 3H), 3.62 (dt, $J=8.5,1.0$ Hz, 1H), 2.85 (dd, $J=16.0,6.0 \mathrm{~Hz}, 1 \mathrm{H}) 2.74$ (dd, $J=16.0,6.0 \mathrm{~Hz}, 1 \mathrm{H}$ ), 0.94 (dt, $J=8.5,1.2 \mathrm{~Hz}, 2 \mathrm{H}$ ), 0.01 (s, 9H) ppm; ${ }^{13} \mathrm{C}$ NMR (125 MHz, $\left.\mathrm{CDCl}_{3}\right) \delta: 171.4,170.9,135.2,128.5,128.3,128.2,97.8,67.1,65.8$, 60.2, 51.9, 34.1, 18.0 ppm; HRMS (ESI) $\mathrm{m} / \mathrm{z}$ calcd. for $\mathrm{C}_{18} \mathrm{H}_{29} \mathrm{NO}_{6} \mathrm{Si}$, [M+Na] $]^{+}$: 406.1656, found: 406.1643. The enantiomeric excess was estimated by chiral HPLC analysis (DAICEL CHIRALPAK IB, eluent: hexane/2-propanol = 99/1, flow rate: $1.0 \mathrm{~mL} / \mathrm{min}$. detector: $\mathrm{UV}$ at $254 \mathrm{~nm}$ ), $t_{\mathrm{R}}=16.4 \mathrm{~min}$ (major), $18.2 \mathrm{~min}$ (minor).

1-tert-Butyl 4-methyl 9H-fluoren-9-ylmethoxycarbonyl-D-aspartate (S3aa): 3aa (25.3 mg $100 \mu \mathrm{mol}$,

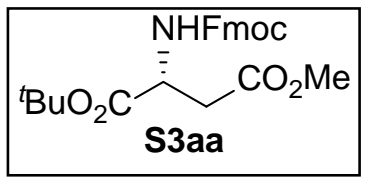
1.0 equiv) was dissolved in $\mathrm{MeOH}(30 \mathrm{~mL})$ and added to $10 \% \mathrm{Pd} / \mathrm{C}(80 \mathrm{mg})$ under an atmosphere of argon. The reaction was carefully flushed with hydrogen gas and stirred at room temperature for $5 \mathrm{~h}$. The atmosphere was replaced with argon before filtration through a pad of Celite ${ }^{\circledR}$. The filtrate was concentrated under reduced pressure and the residue was dissolved in $\mathrm{CH}_{2} \mathrm{Cl}_{2}\left(1.0 \mathrm{~mL}\right.$ ). To the solution were added ${ }^{i} \mathrm{Pr}_{2} \mathrm{NEt}$ (64.6 mg, $500 \mu \mathrm{mol}, 5.0$ equiv) and FmocCl (25.8 mg, $120 \mu \mathrm{mol}, 1.2$ equiv). The resulting mixture was stirred at ambient temperature for 17 $\mathrm{h}$, then the solvent was evaporated. The residue was purified by silica-gel column chromatography (eluent: 
hexane/EtOAc, 1:1) to afford S3aa as colorless oil (26.0 mg, $61 \mu \mathrm{mol}, 61 \%)$.

$[\alpha]_{D}^{23}-7.1$ ( с $\left.0.73, \mathrm{CHCl}_{3}\right)$; IR (neat) $\tilde{v}: 3368,1721 \mathrm{~cm}^{-1} ;{ }^{1} \mathrm{H}$ NMR $\left(500 \mathrm{MHz}, \mathrm{CDCl}_{3}\right) \delta: 7.75$ (d, $J=7.5$ Hz, 2H), 7.60 (d, $J=7.5 \mathrm{~Hz}, 2 \mathrm{H}), 7.40$ (t, $J=7.5 \mathrm{~Hz}, 2 \mathrm{H}$ ), 5.80 (d, $J=8.5 \mathrm{~Hz}, 1 \mathrm{H}), 4.54$ (dt, $J=8.5,4.5$ Hz, 1H), 4.38 (dt, $J=17.0,7.0 \mathrm{~Hz}, 2 \mathrm{H}), 4.23$ (t, $J=7.0 \mathrm{~Hz}, 1 \mathrm{H}), 3.71$ (s, 3H), 3.00 (dd, $J=17.0,4.0 \mathrm{~Hz}$, 1H), 2.84 (dd, $J=17.0,4.0 \mathrm{~Hz}, 1 \mathrm{H}), 1.48$ (s, 9H) ppm; ${ }^{13} \mathrm{C}$ NMR (125 MHz, $\left.\mathrm{CDCl}_{3}\right) \delta: 171.3,169.6,156.0$, 143.9, 143.8, 141.3, 127.8, 127.1, 125.2, 120.0, 82.7, 67.2, 52.0, 51.0, 47.2, 36.8, 27.9 ppm; HRMS (ESI) $\mathrm{m} / \mathrm{z}$ calcd. for 448.1731 , [M+Na] ${ }^{+}$found: 448.1757 . The enantiomeric excess was estimated by chiral HPLC analysis (DAICEL CHIRALPAK IA, eluent: hexane/2-propanol = 96/4, flow rate: $1.0 \mathrm{~mL} / \mathrm{min}$. detector: $\mathrm{UV}$ at $254 \mathrm{~nm}$ ), $t_{\mathrm{R}}=20.3 \mathrm{~min}$ (major), $26.6 \mathrm{~min}$ (minor).

\section{(D) O-Deprotection and KAHA Ligation}

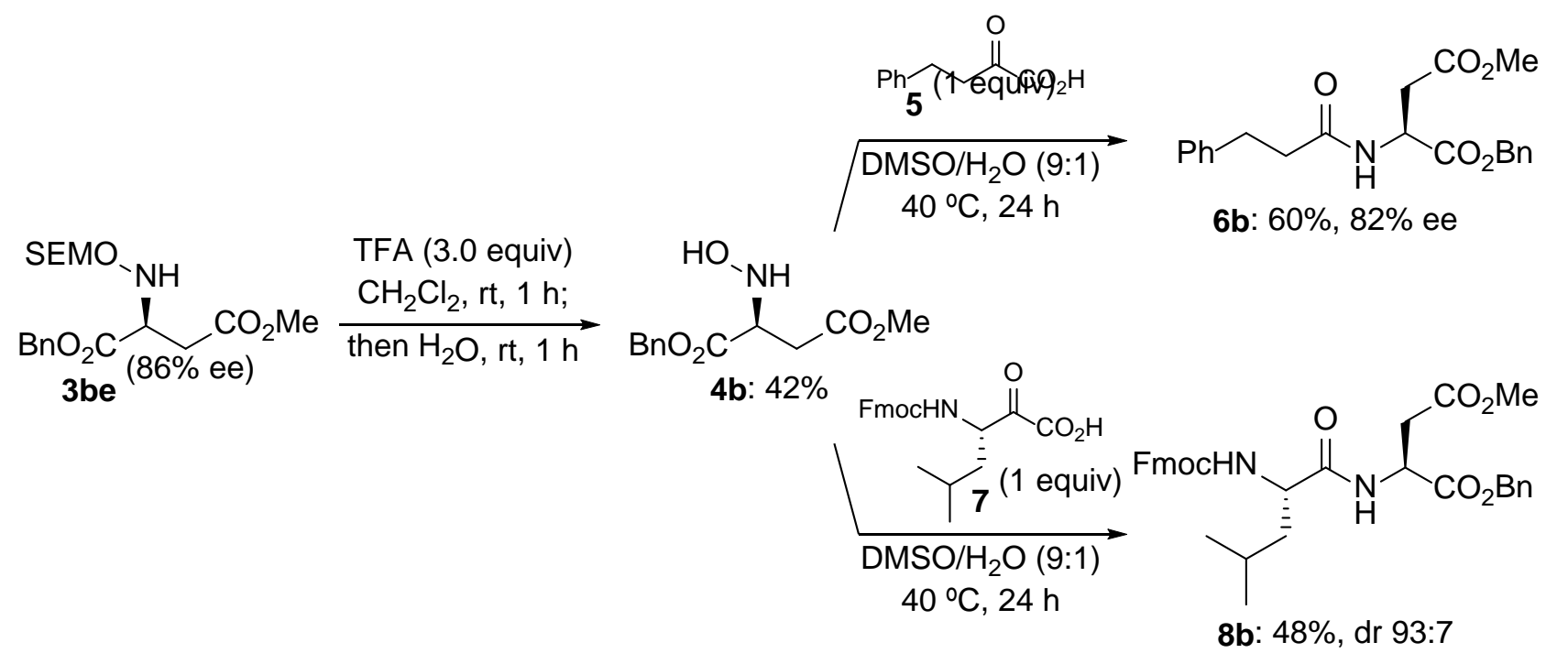

1-Benzyl 4-methyl $N$-hydroxy-L-aspartate (4b): To a solution of 3be (192.7 mg, $500 \mu \mathrm{mol}, 1.0$ equiv) in $\mathrm{CH}_{2} \mathrm{Cl}_{2}$ (5 mL) was added TFA (172.5 mg, $1.5 \mathrm{mmol}, 3.0$ equiv) at room temperature and stirred for $1 \mathrm{~h}$. $\mathrm{H}_{2} \mathrm{O}$ (5 mL) was added and further stirred at ambient temperature for $1 \mathrm{~h}$, and the mixture was dried over $\mathrm{Na}_{2} \mathrm{SO}_{4}$. The solids were filtered off and the solvent was removed under reduced presssure. The residue was then purified by silica-gel column chromatography (eluent: hexane/EtOAc, 1:2) to afford $\mathbf{4 b}$ as colorless oil (53.1 mg, $210 \mu \mathrm{mol}, \mathbf{4 2} \%$ ). Since $\mathbf{4 b}$ was gradually degraded on standing, $\mathbf{4 b}$ was used for KAHA ligation soon after purification.

${ }^{1} \mathrm{H}$ NMR (500 MHz, $\mathrm{CDCl}_{3}$ ) $\delta: 7.32$ (m, 5H), 5.22 (s, 2H), 4.06 (dt, J = 8.0, $5.0 \mathrm{~Hz}, 1 \mathrm{H}$ ), 3.66 (s, 3H), 2.87 (dd, $J=16.0,5.0 \mathrm{~Hz}, 1 \mathrm{H}), 2.80$ (dd, $J=16.0,5.0 \mathrm{~Hz}, 1 \mathrm{H}$ ) ppm.

1-Benzyl 4-methyl (3-phenylpropanoyl)-L-aspartate (6b): A mixture of 4b (23.3 mg, 92 mol, 1.0 equiv)

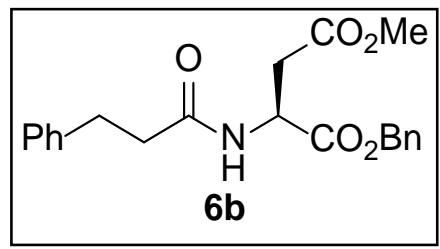
and 2-oxo-4-phenylbutanoic acid (5) (16.3 mg, $92 \mu \mathrm{mol}, 1.0$ equiv) in $\mathrm{DMSO} / \mathrm{H}_{2} \mathrm{O}(9: 1,200 \mu \mathrm{L})$ was stirred at $40{ }^{\circ} \mathrm{C}$ for $24 \mathrm{~h}$. The crude mixture was extracted with EtOAc ( $2 \mathrm{~mL}, 3$ times) and then purified by silica-gel column chromatography (eluent: hexane/EtOAc, 1:2) to afford to $\mathbf{6 b}$ as yellow oil (26.1

mg, $70 \mu \mathrm{mol}, 60 \%$, 82\% ee).

$[\alpha]_{D}^{24}-13.2$ (c 1.75, $\left.\mathrm{CHCl}_{3}\right)$; IR (neat) $\tilde{v}: 3311,1732,1652 \mathrm{~cm}^{-1} ;{ }^{1} \mathrm{H} \mathrm{NMR}\left(500 \mathrm{MHz}, \mathrm{CDCl}_{3}\right) \delta:$ 7.38-7.30 
(m, 6H), 7.27-7.24 (m, 1H), 7.19-7.17 (m, 3H), 6.42 (d, $J=8.0 \mathrm{~Hz}, 1 \mathrm{H}), 5.17$ (dd, $J=28.0,12.5 \mathrm{~Hz}, 2 \mathrm{H}$ ), 4.88 (dt, $J=8.0,4.0 \mathrm{~Hz}, 1 \mathrm{H}$ ), 3.00 (dd, $J=17.5,4.0 \mathrm{~Hz}, 1 \mathrm{H}), 2.95$ (t, $J=8.0 \mathrm{~Hz}, 2 \mathrm{H}$ ), 2.75 (dd, $J=17.5$, $4.0 \mathrm{~Hz}, 1 \mathrm{H}$ ), 2.59 (ddd, $J=18.0,15.0,8.0 \mathrm{~Hz}, 1 \mathrm{H}$ ), 2.49 (ddd, $J=18.0 \mathrm{~Hz}, 15.0,8.0 \mathrm{~Hz}$ ) ppm; ${ }^{13} \mathrm{C}$ NMR (125 MHz, $\left.\mathrm{CDCl}_{3}\right) \delta: 171.8,171.4,170.5,140.5,135.1,128.55,128.45,128.26,126.1,67.5,51.9$, 48.3, 38.0, 36.0, 31.4 ppm; HRMS (ESI) $m / z$ calcd. For $\mathrm{C}_{21} \mathrm{H}_{23} \mathrm{NO}_{5},[\mathrm{M}+\mathrm{H}]^{+}$: 370.1649, found: 370.1676. The enantiomeric excess was estimated by chiral HPLC analysis (DAICEL CHIRALPAK IB, eluent: hexane/2propanol = 99/1, flow rate: $1 \mathrm{~mL} / \mathrm{min}$. detector: $\mathrm{UV}$ at $254 \mathrm{~nm}$ ), $t_{\mathrm{R}}=18.2 \mathrm{~min}$ (minor), 16.4 min (major).

1-Benzyl 4-methyl 9H-fluoren-9-ylmethoxycarbonyl-L-leucyl-L-aspartate (8b): A mixture of 4 b (60.1

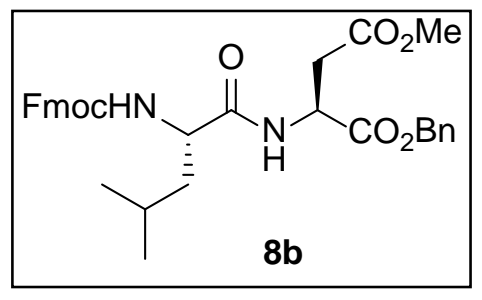
mg, $156 \quad \mu$ mol, $1.0 \quad$ equiv) and (S)-3-(9H-fluoren-9ylmethoxycarbonyl)amino)-5-methyl-2-oxohexanoic acid (7) (57.1 mg, 150 $\mu$ mol, 1.0 equiv) in DMSO/ $\mathrm{H}_{2} \mathrm{O}(9: 1,15 \mathrm{~mL})$ was stirred at $40{ }^{\circ} \mathrm{C}$ for $15 \mathrm{~h}$. The crude mixture was extracted with EtOAc ( $2 \mathrm{~mL}, 3$ times) and then purified by silica-gel column chromatography (eluent: hexane/EtOAc, 1:2) to afford to $\mathbf{8 b}$ as yellow oil (41.2 $\mathrm{mg}, 72 \mu \mathrm{mol}, 50 \%, 93: 7 \mathrm{dr}$ ).

$[\alpha]_{D}^{23}-3.20$ (c 1.20, $\mathrm{CHCl}_{3}$ ); IR (neat) $\tilde{v}: 3314,1738,1661 \mathrm{~cm}^{-1} ;{ }^{1} \mathrm{H}$ NMR (500 MHz, CDCl 3 ) $\delta: 7.75$ (d, $J=7.0 \mathrm{~Hz}, 2 \mathrm{H}), 7.57$ (d, $J=5.5 \mathrm{~Hz}, 2 \mathrm{H}), 7.39-7.29$ (m, 9H), 6.95 (d, $J=8.0 \mathrm{~Hz}, 1 \mathrm{H}), 5.34$ (d, $J=8.0 \mathrm{~Hz}$, 1H), 5.15 (dd, $J=21.0,12.0 \mathrm{~Hz}, 2 \mathrm{H}), 4.91$ (t, $J=4.0 \mathrm{~Hz}, 1 \mathrm{H}), 4.36(\mathrm{~m}, 2 \mathrm{H}) 4.21(\mathrm{~m}, 2 \mathrm{H}), 3.58$ (s, 3H), 3.05 (dd, $J=17.0,3.5 \mathrm{~Hz}, 1 \mathrm{H}), 2.81$ (dd, $J=17.0,3.5 \mathrm{~Hz}, 1 \mathrm{H}), 1.63$ (m, 2H), 1.50 (m, 1H), 0.89 (s, 6H) ppm; ${ }^{13} \mathrm{C}$ NMR (125 MHz, $\left.\mathrm{CDCl}_{3}\right) \delta: 172.2,171.5,170.3,156.1,143.9,143.8,141.3,135.1,128.69,128.60$, 128.4, 127.8, 127.1, 125.1, 120.0, 67.7, 67.1, 53.4, 52.1, 48.5, 41.9, 36.0, 24.6, 22.9, 22.0 ppm; HRMS (ESI) $\mathrm{m} / \mathrm{z}$ calcd. For $\mathrm{C}_{33} \mathrm{H}_{36} \mathrm{~N}_{2} \mathrm{O}_{7},[\mathrm{M}+\mathrm{Na}]^{+}$: 595.2415, found: 595.2396. 


\section{(E) References}

[1] N. Hayama, T. Azuma, Y. Kobayashi, Y. Takemoto, Chem. Pharm. Bull., 2016, 64, 704.

[2] S. E. Denmark, A. Thorarensen, D. S. Middleton, J. Am. Chem. Soc., 1996, 118, 8266.

[3] D. Hadjipavlou-Litina, G. E. Magoulas, M. Krokidis, D. Papaioannou, Eur. J. Med. Chem., 2010, 45, 298.

[4] J. P. D. van Veldhoven, C. C. Blad, A. C. Klopman, D. R. Wolfram, M. J. Abdelkadir, J. R. Lane, J. Brussee, A. P. IJzerman, Bioorg. Med. Chem. Lett., 2011, 21, 2736.

[5] Ö. D. Ekici, Z. Z. Li, A. J. Campbell, K. E. James, J. L. Asgian, J. Mikolajczyk, G. S. Salvesen, R. Ganesan, S. Jelakovic, M. G. Grütter, J. C. Powers, J. Med. Chem., 2006, 49, 5728.

[6] A. Odriozola, M. Oiarbide, C. Palomo, Chem. Eur. J., 2017, 23, 12758.

[7] L. A. Carpino, C. A. Giza, B. A. Carpino, J. Am. Chem. Soc., 1959, 81, 955. 
(F) ${ }^{1} \mathrm{H}$ NMR and ${ }^{13} \mathrm{C}$ NMR Spectra

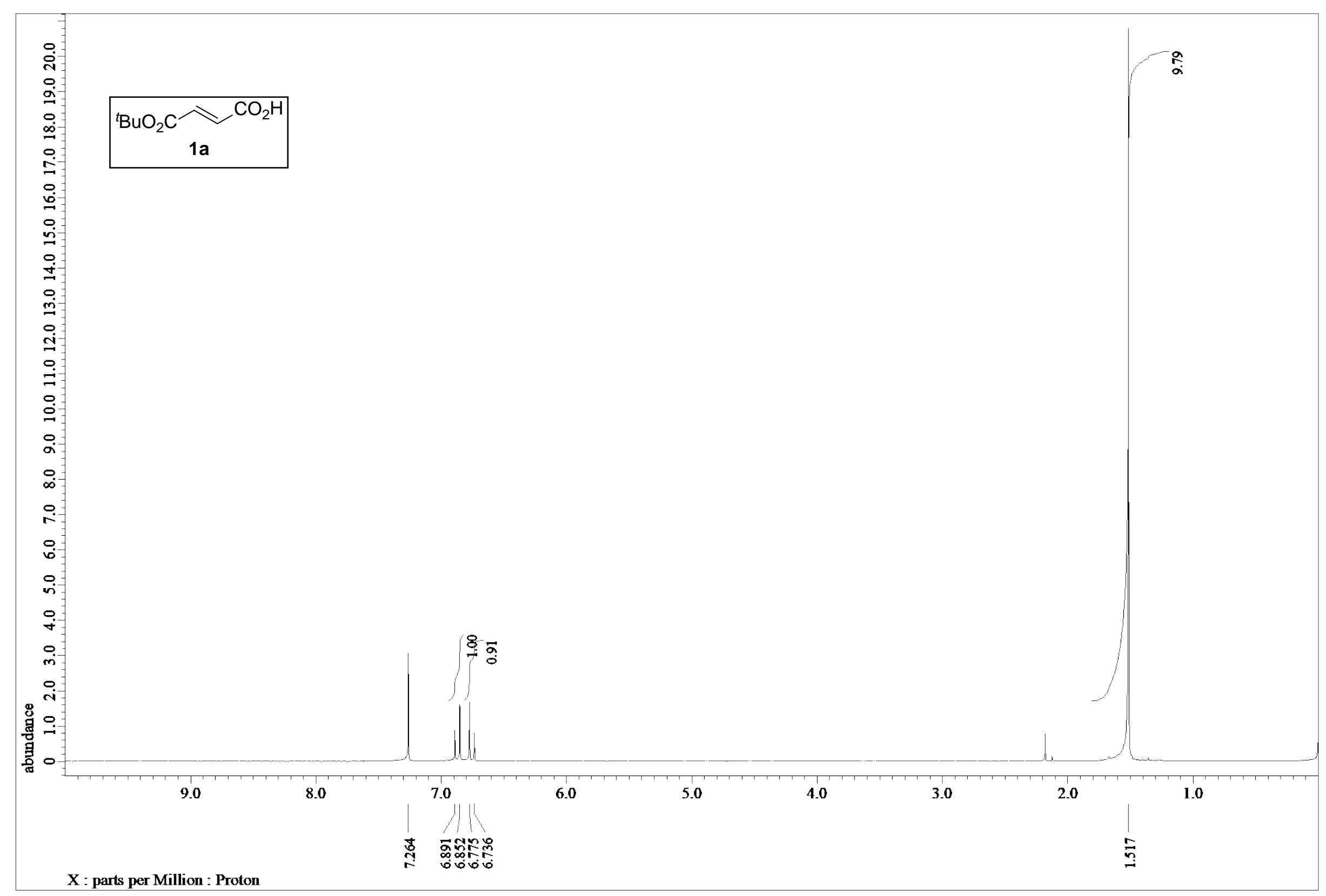




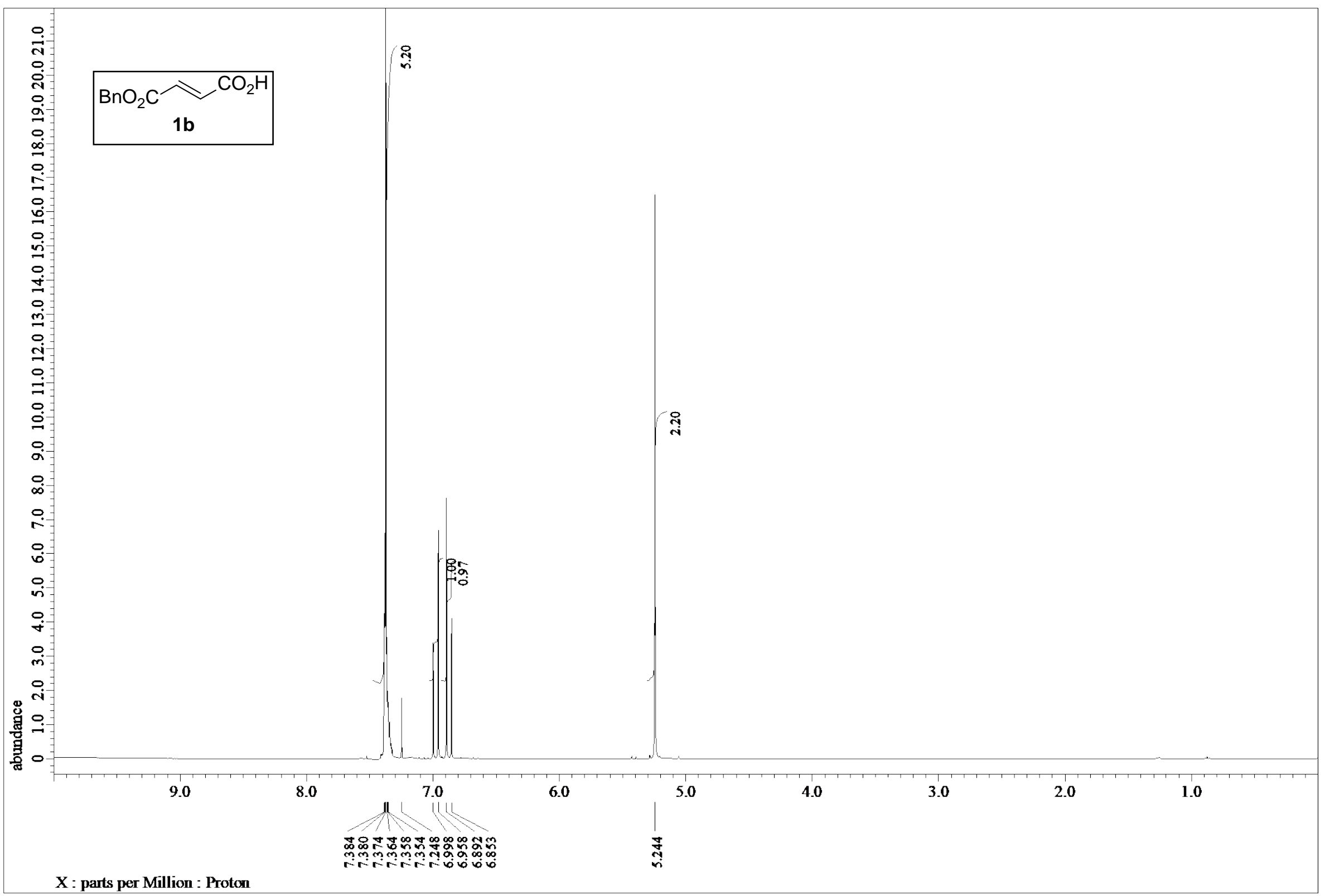




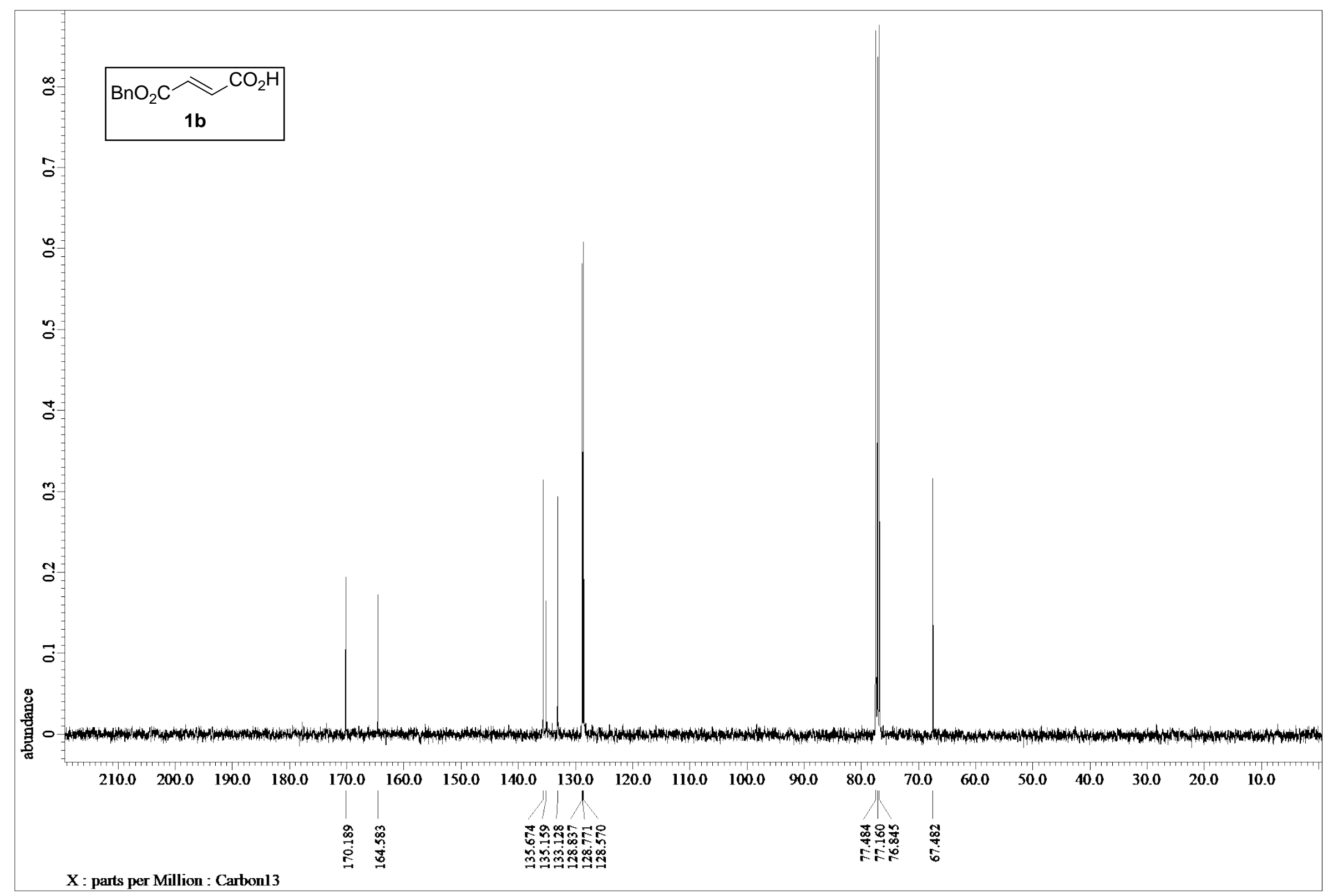




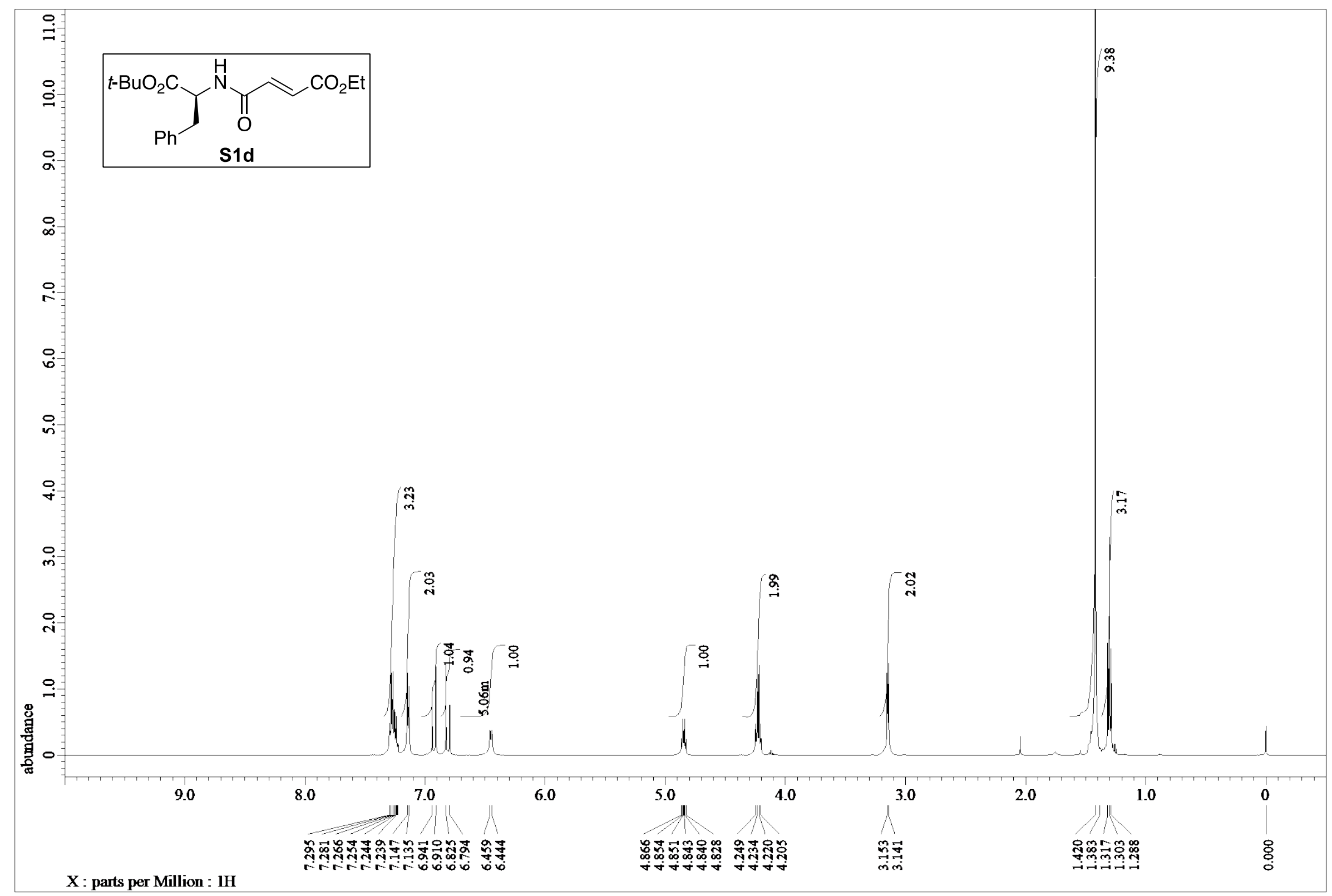




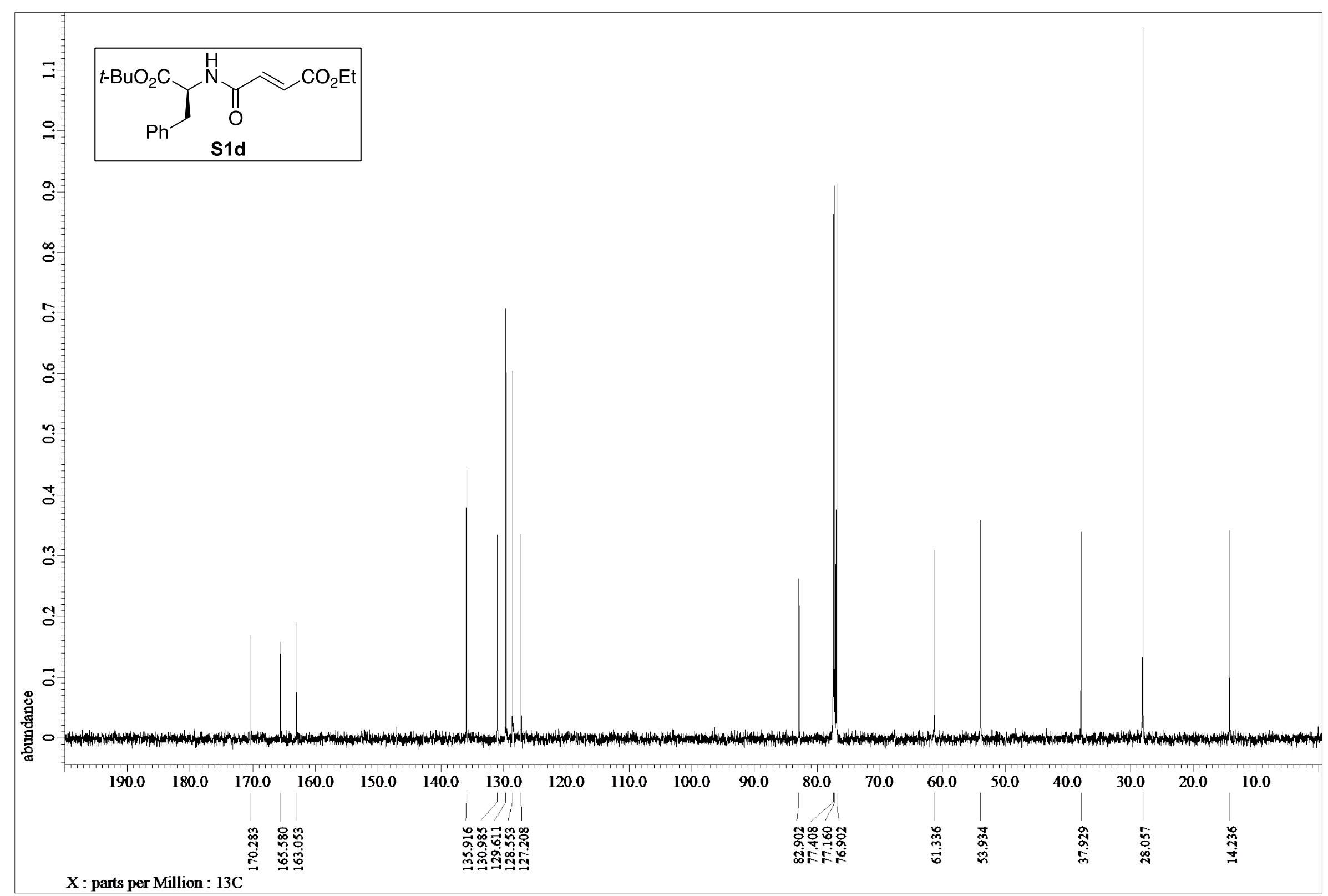




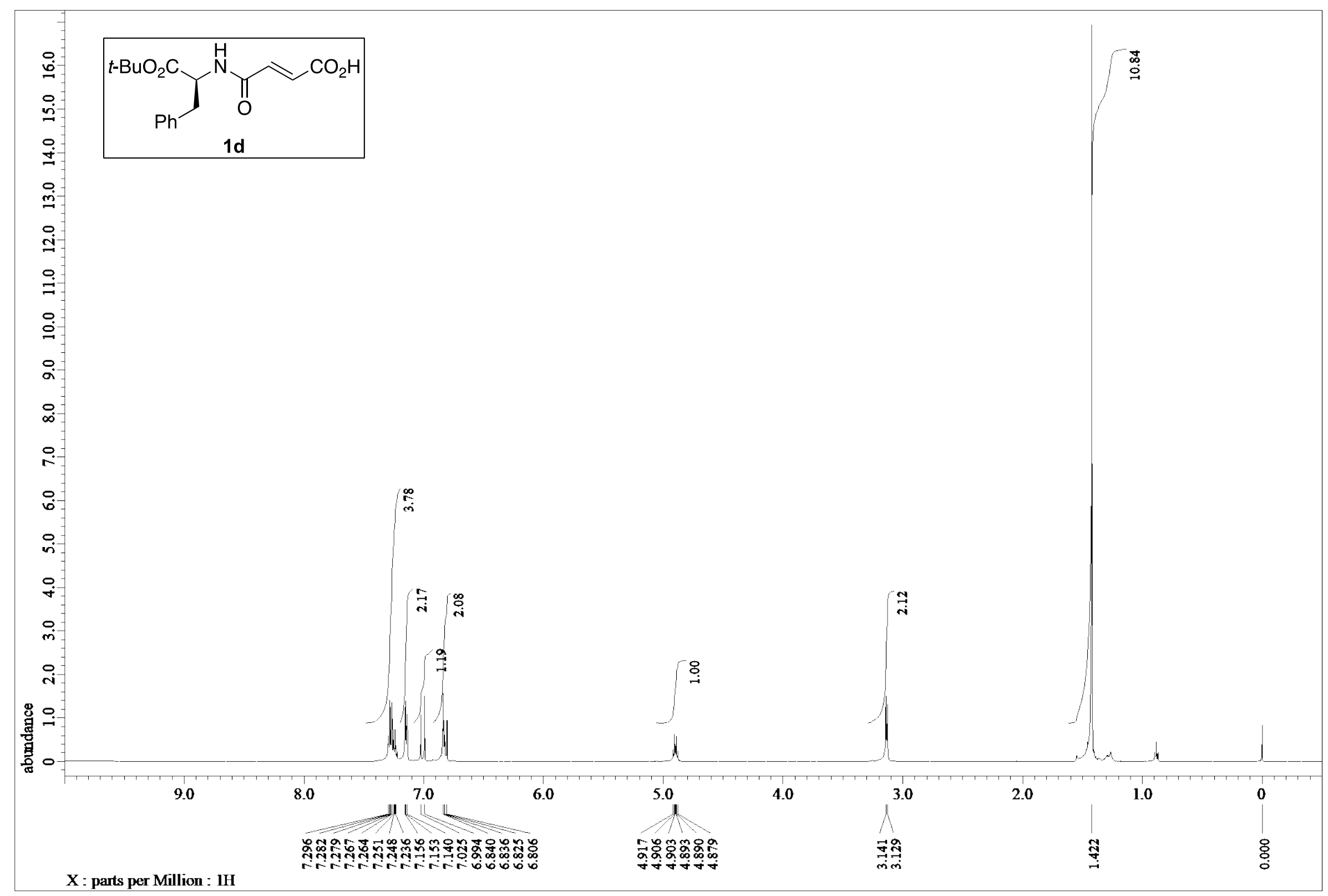




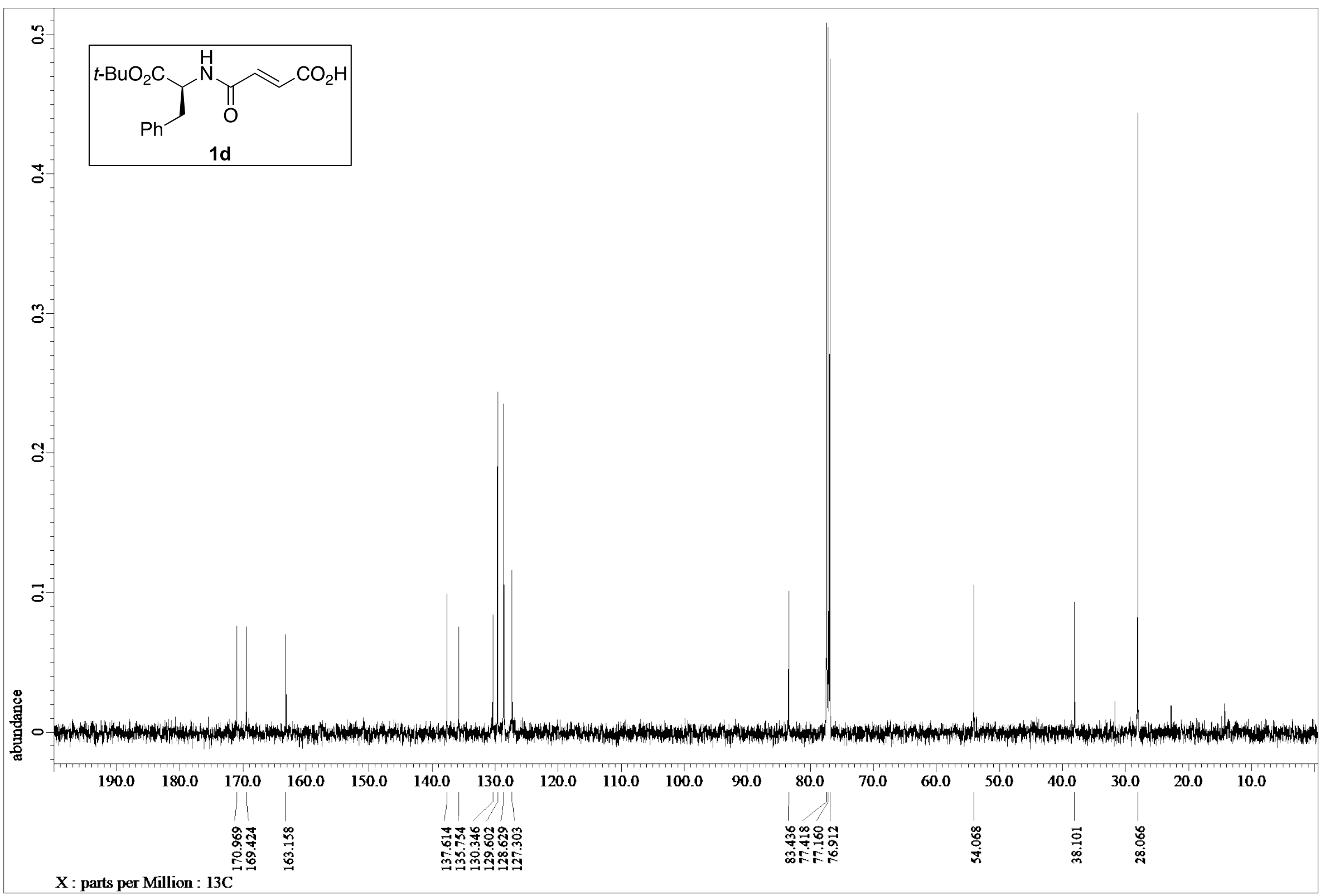




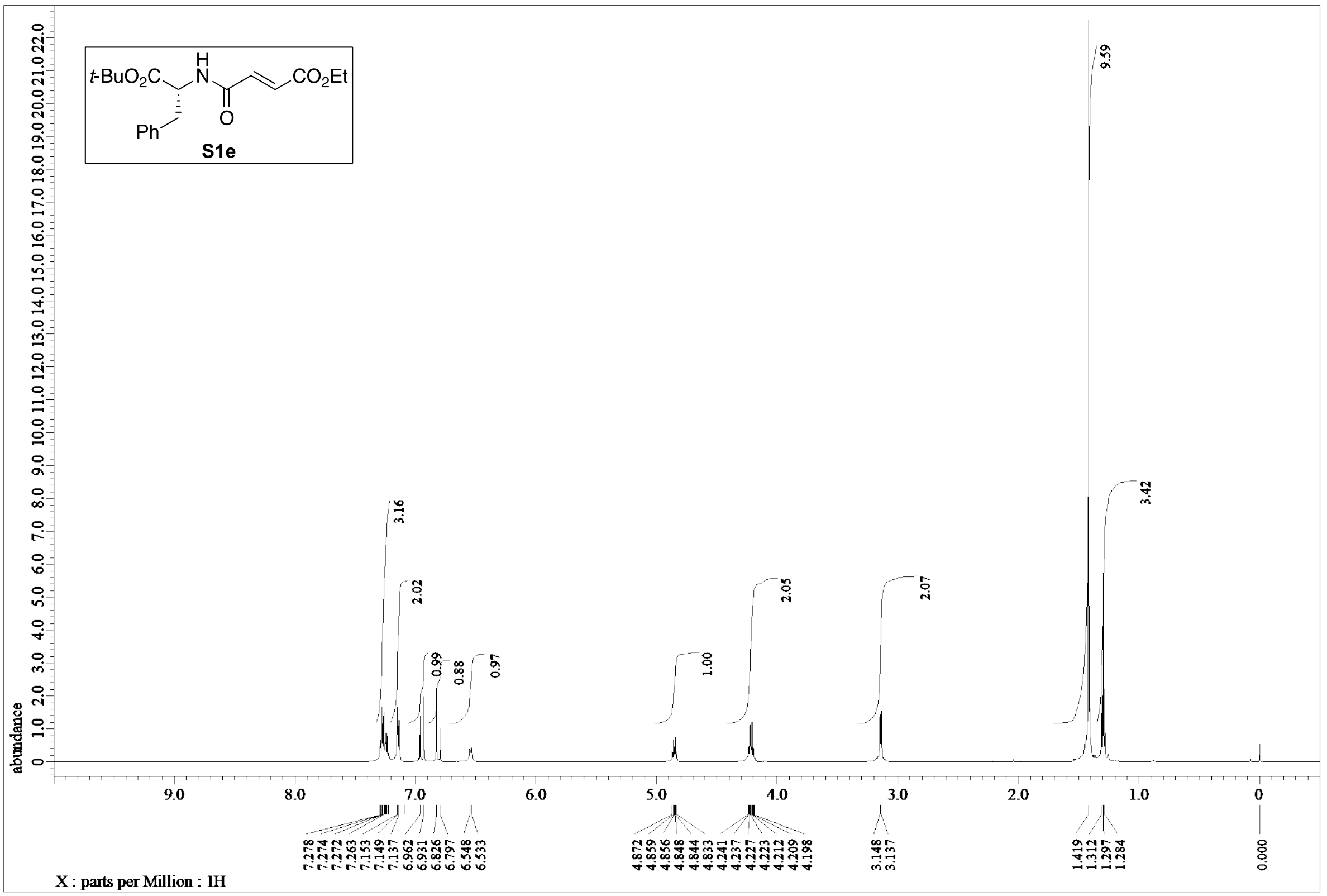




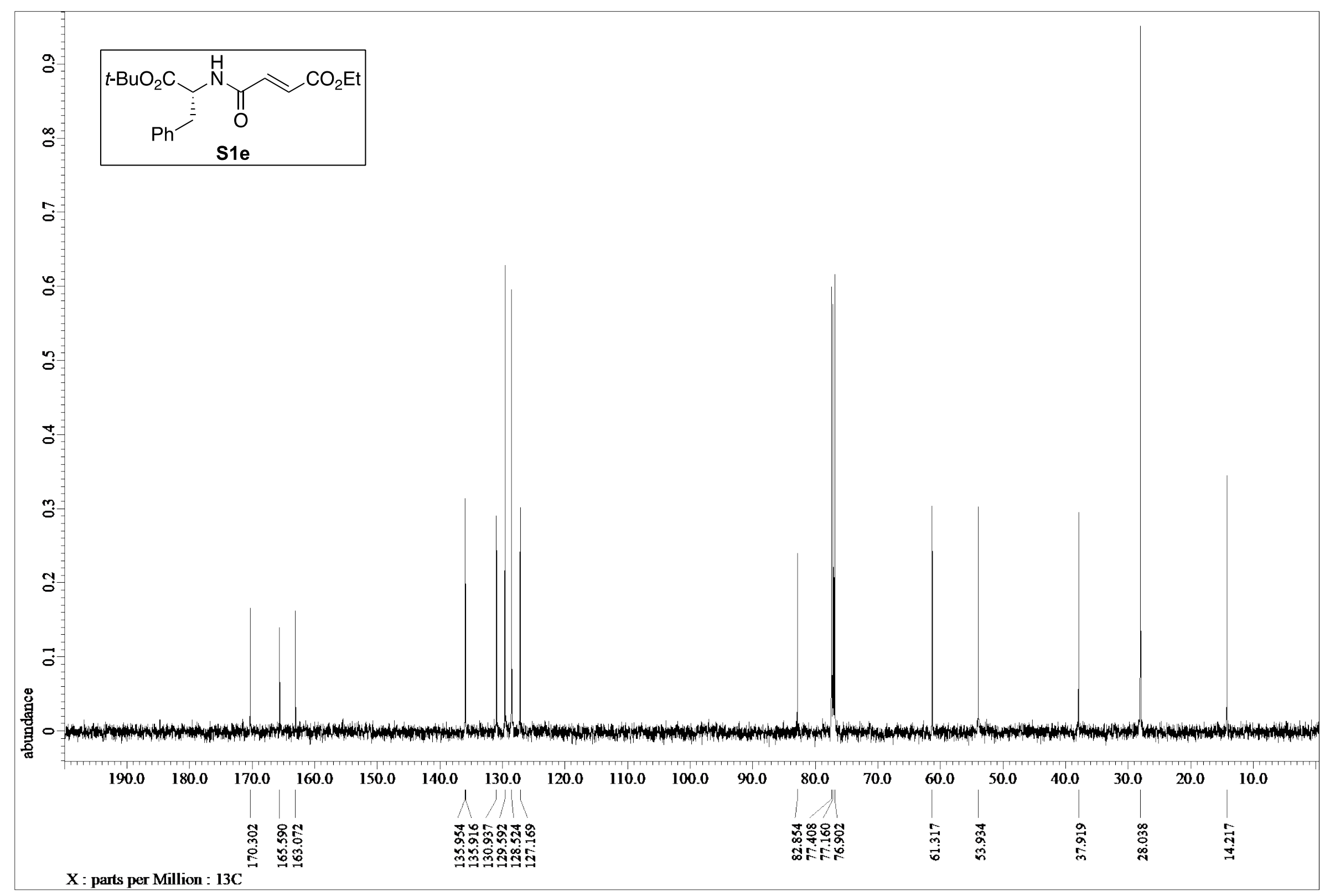




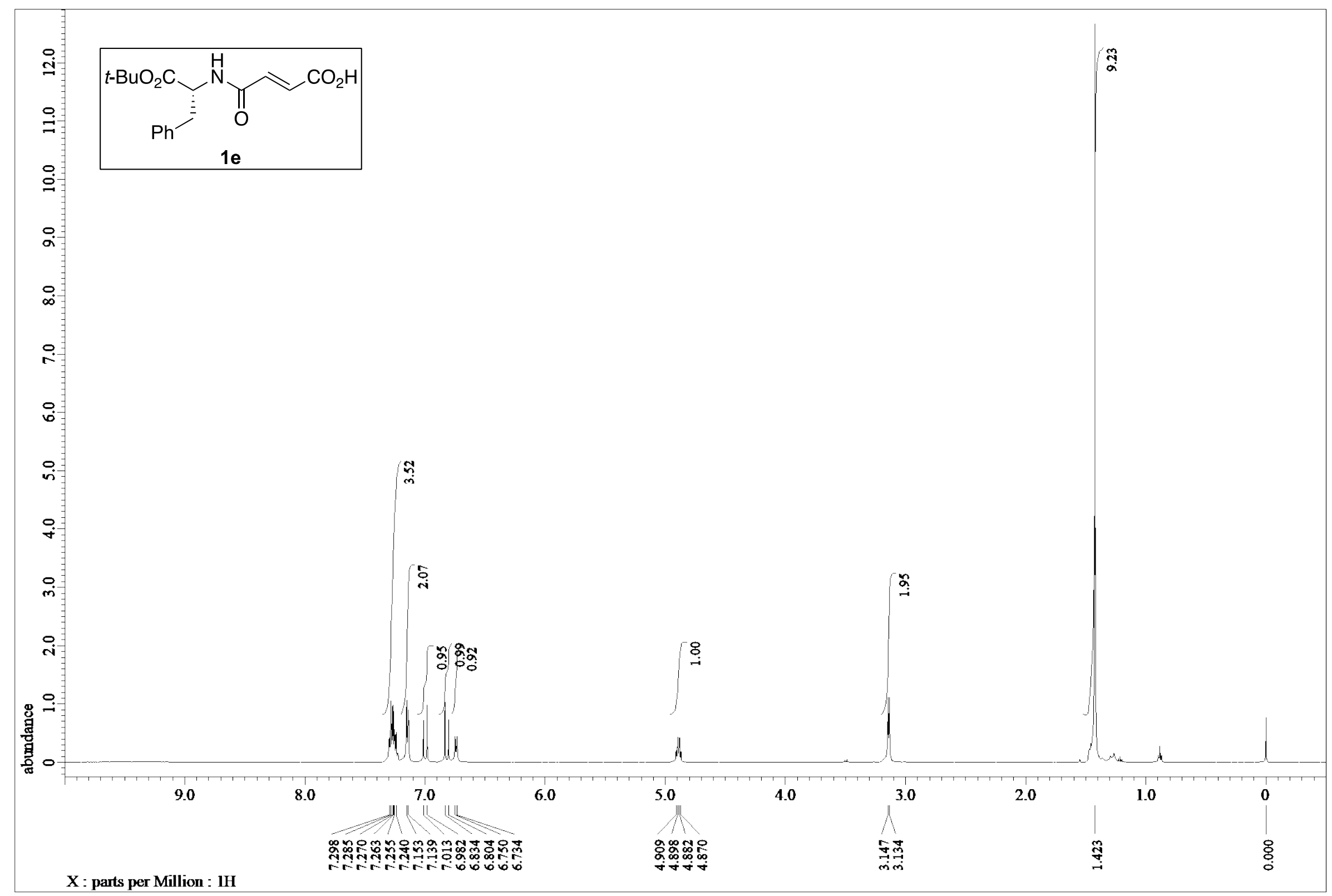




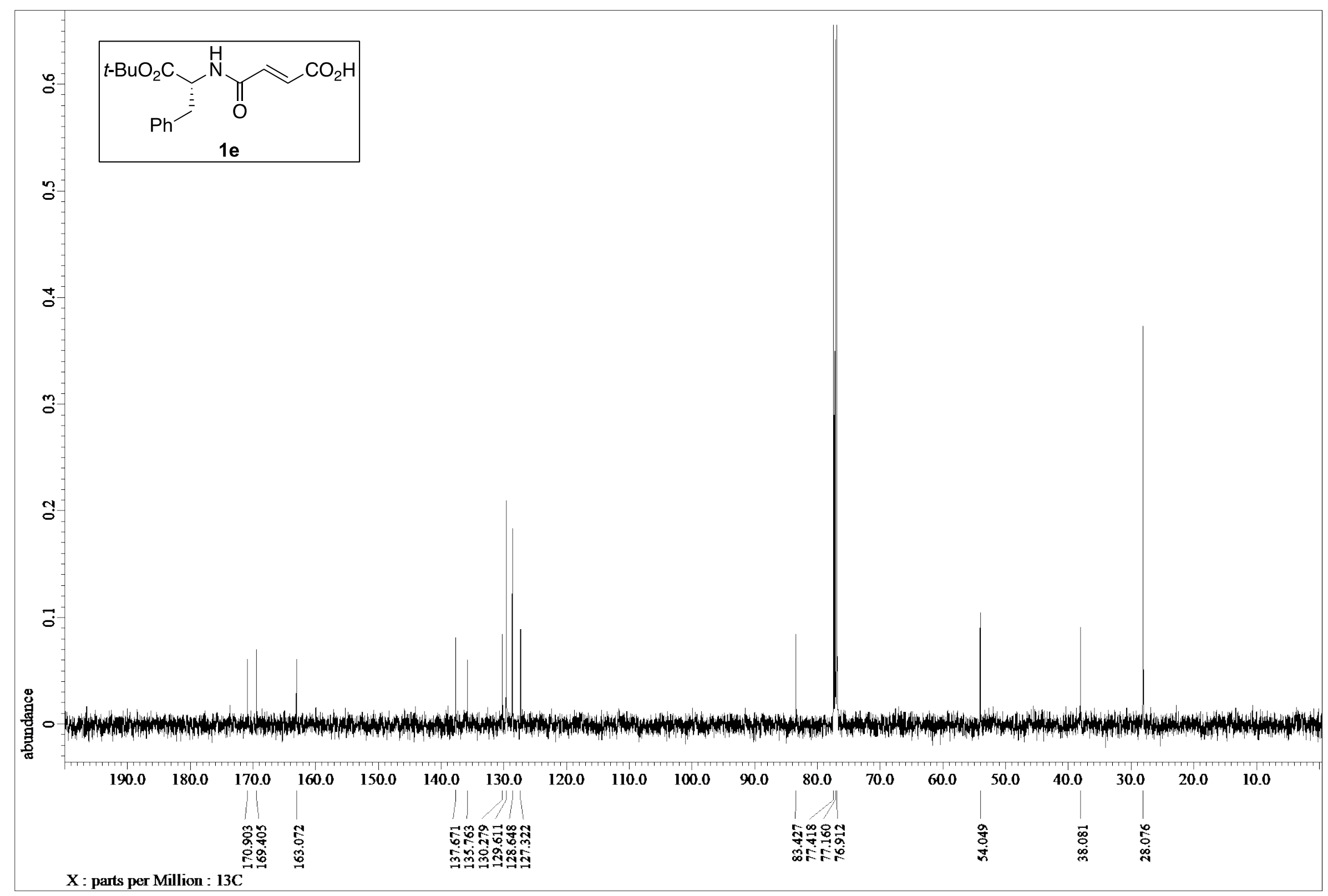




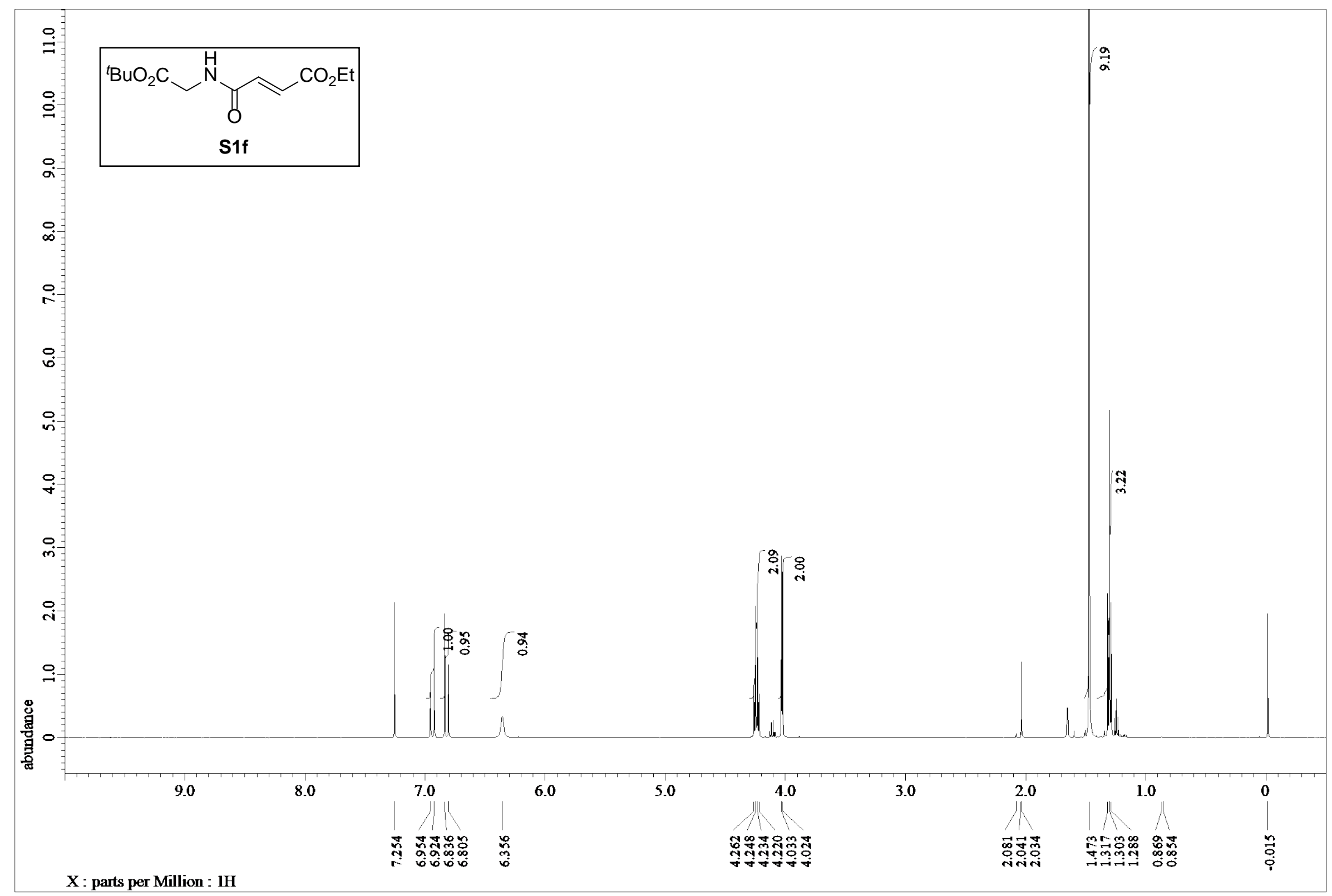




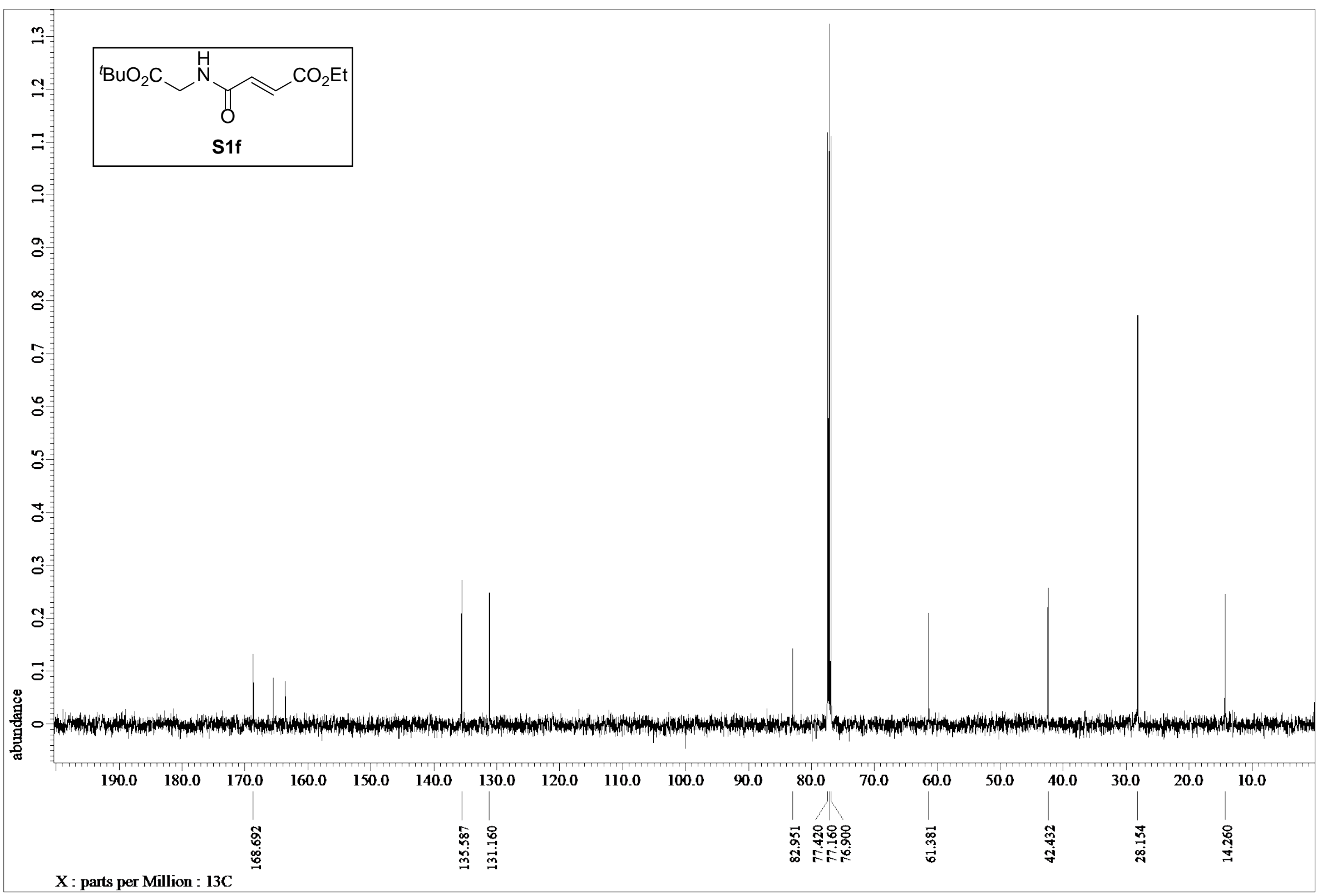




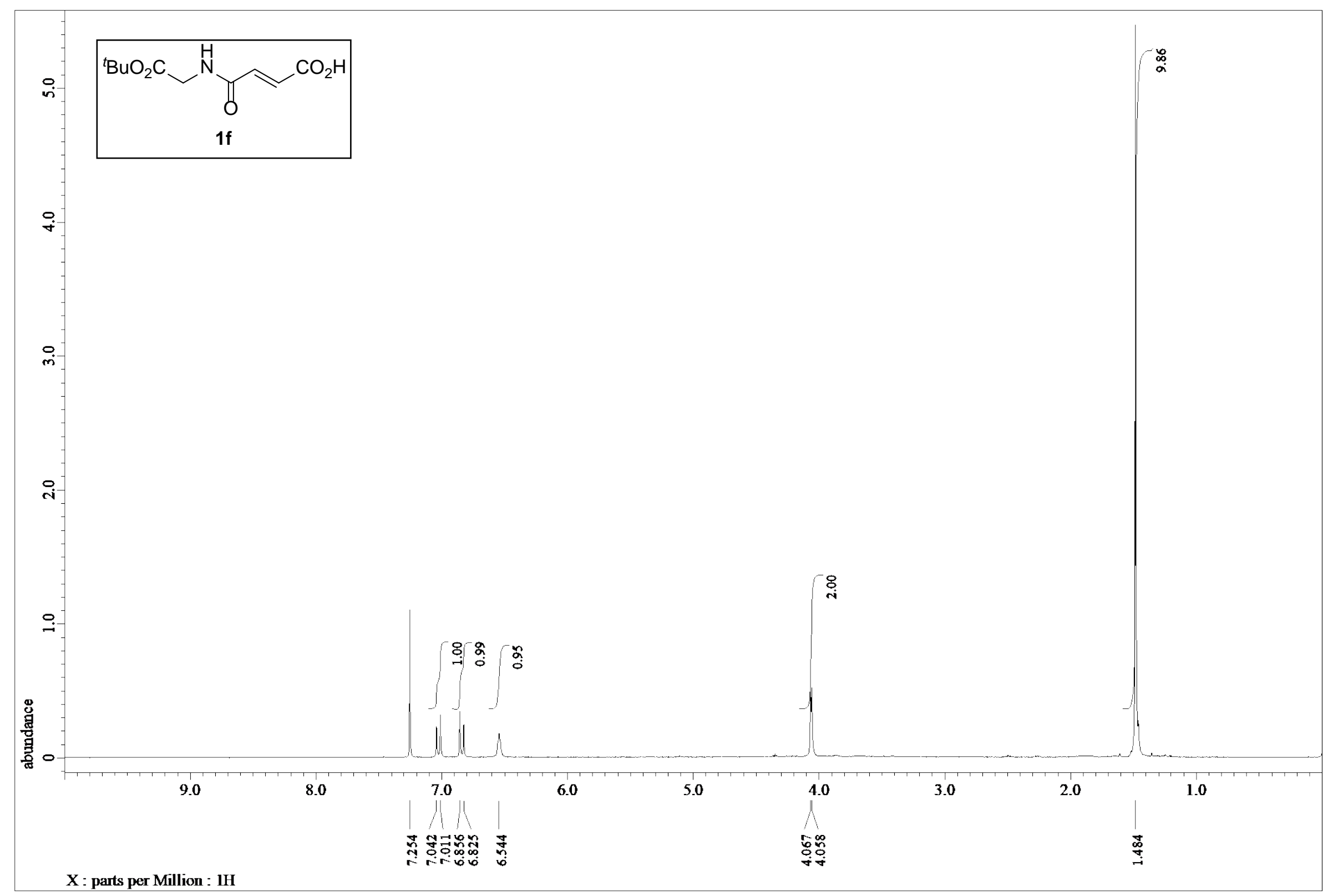




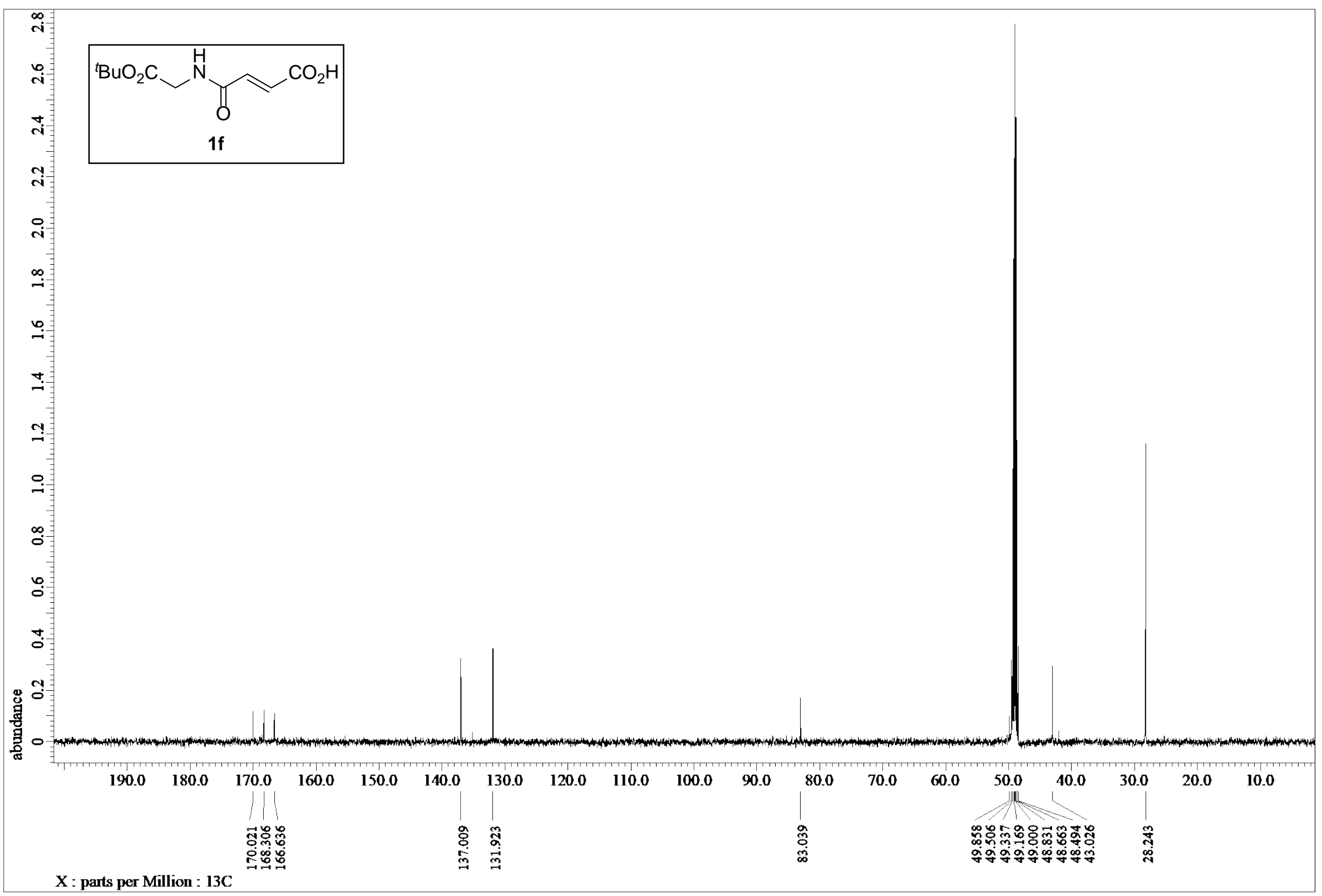




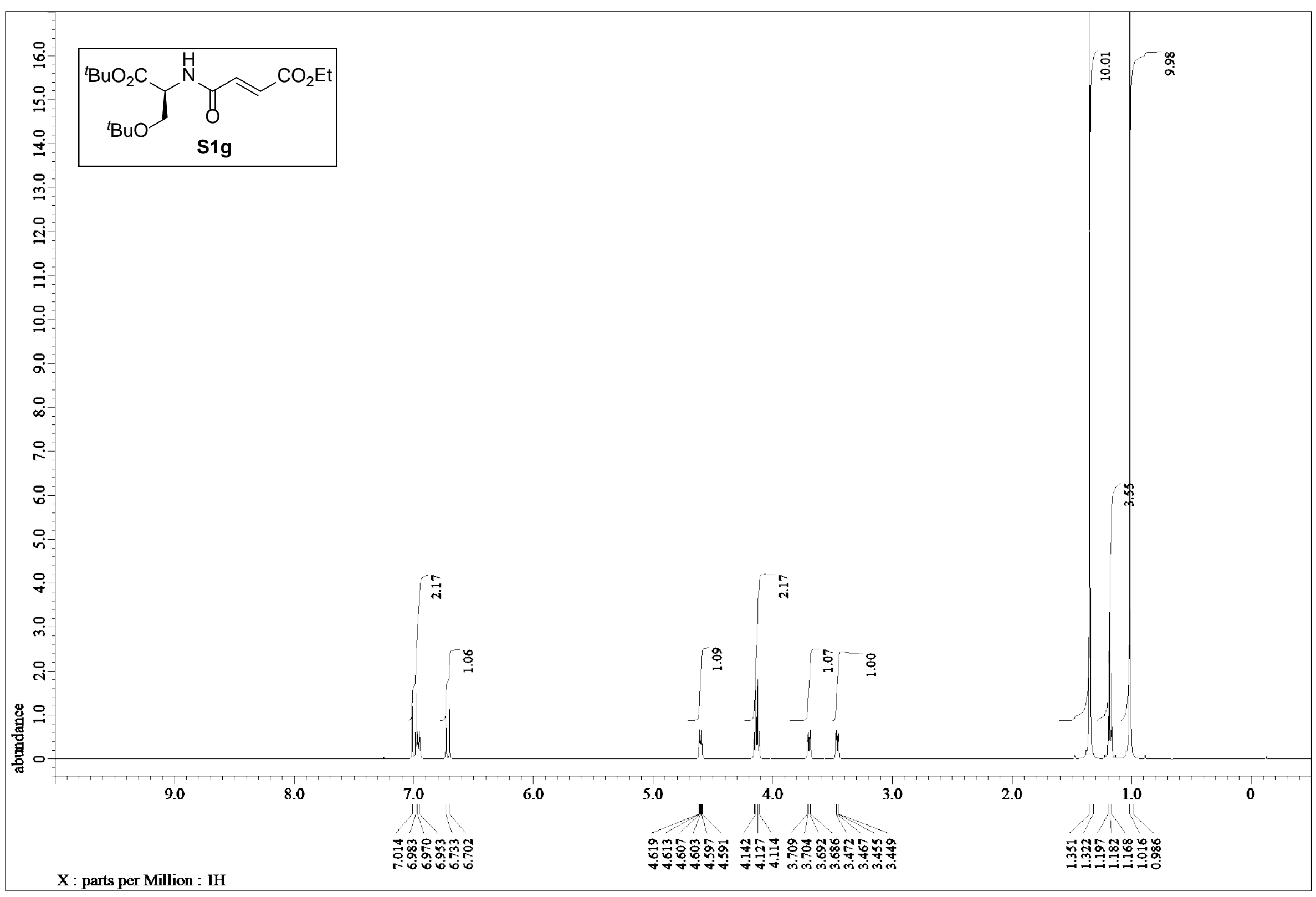




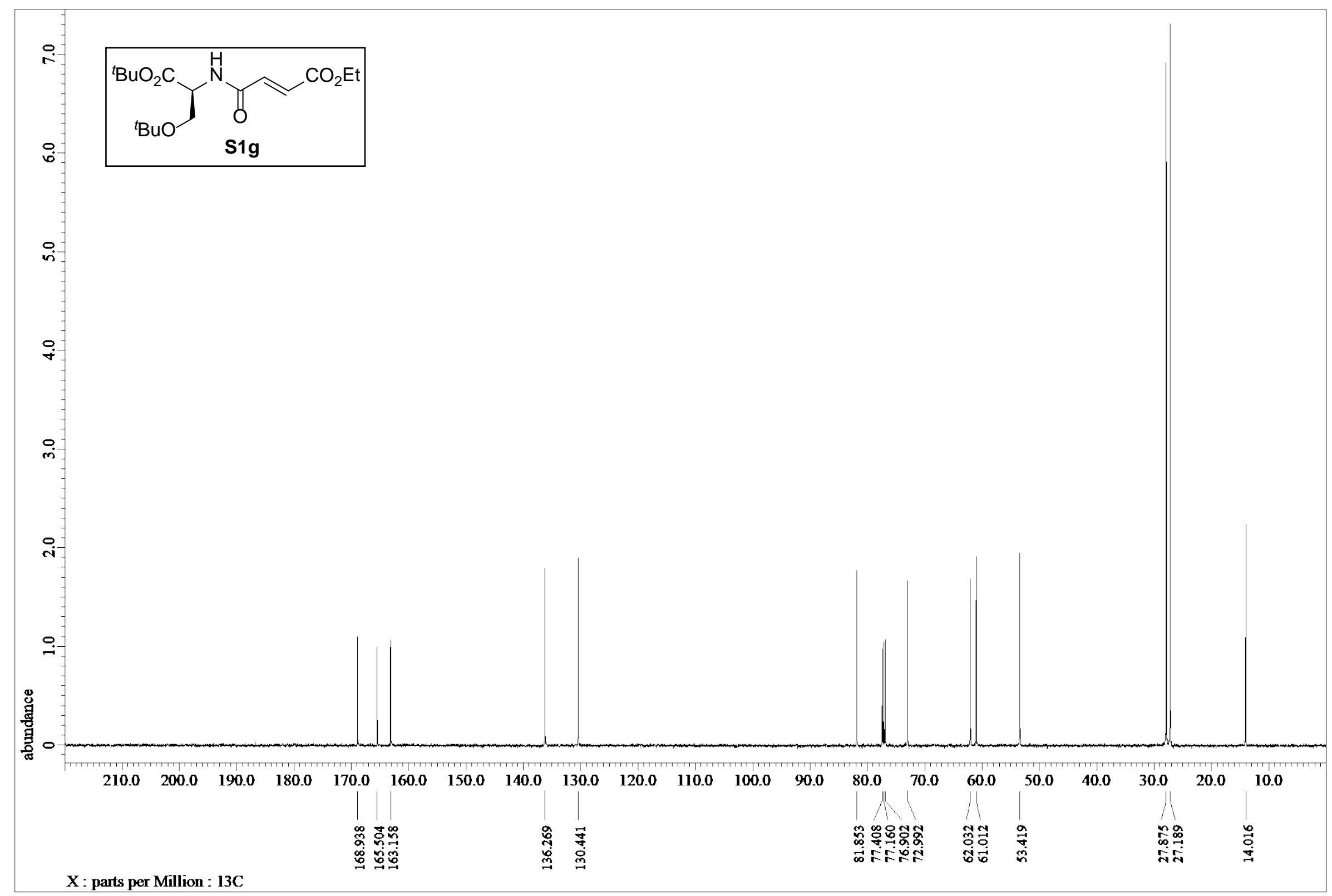




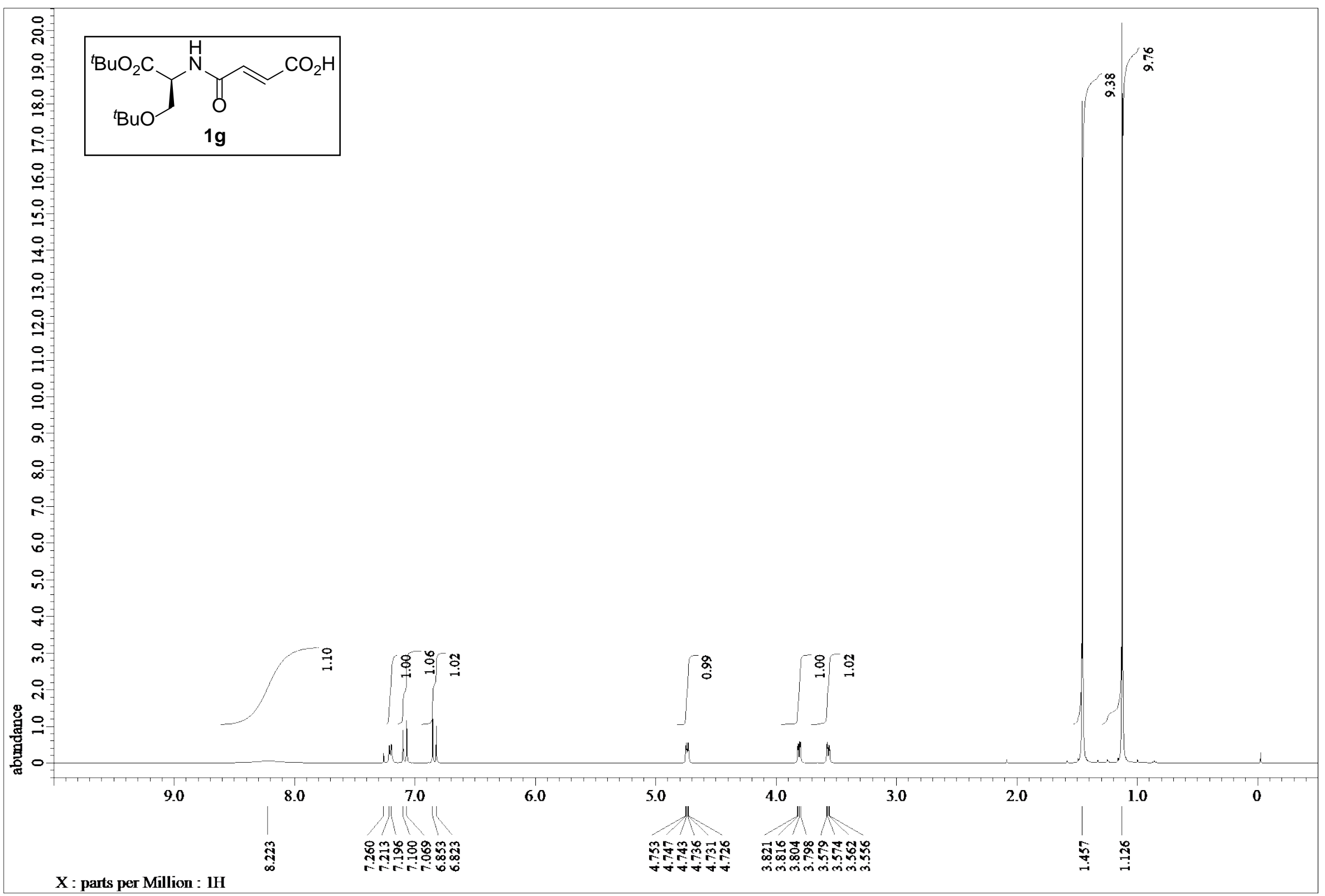




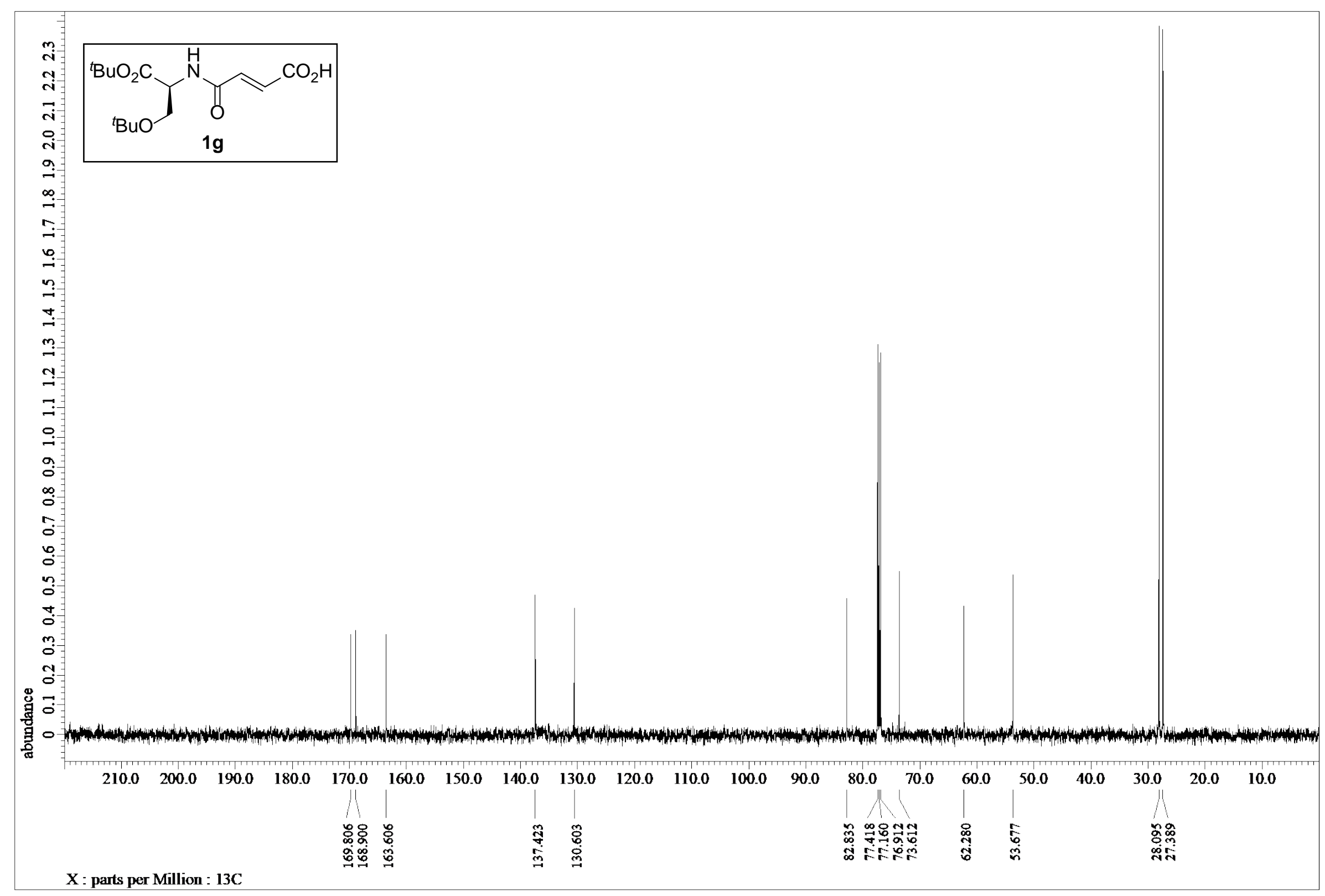




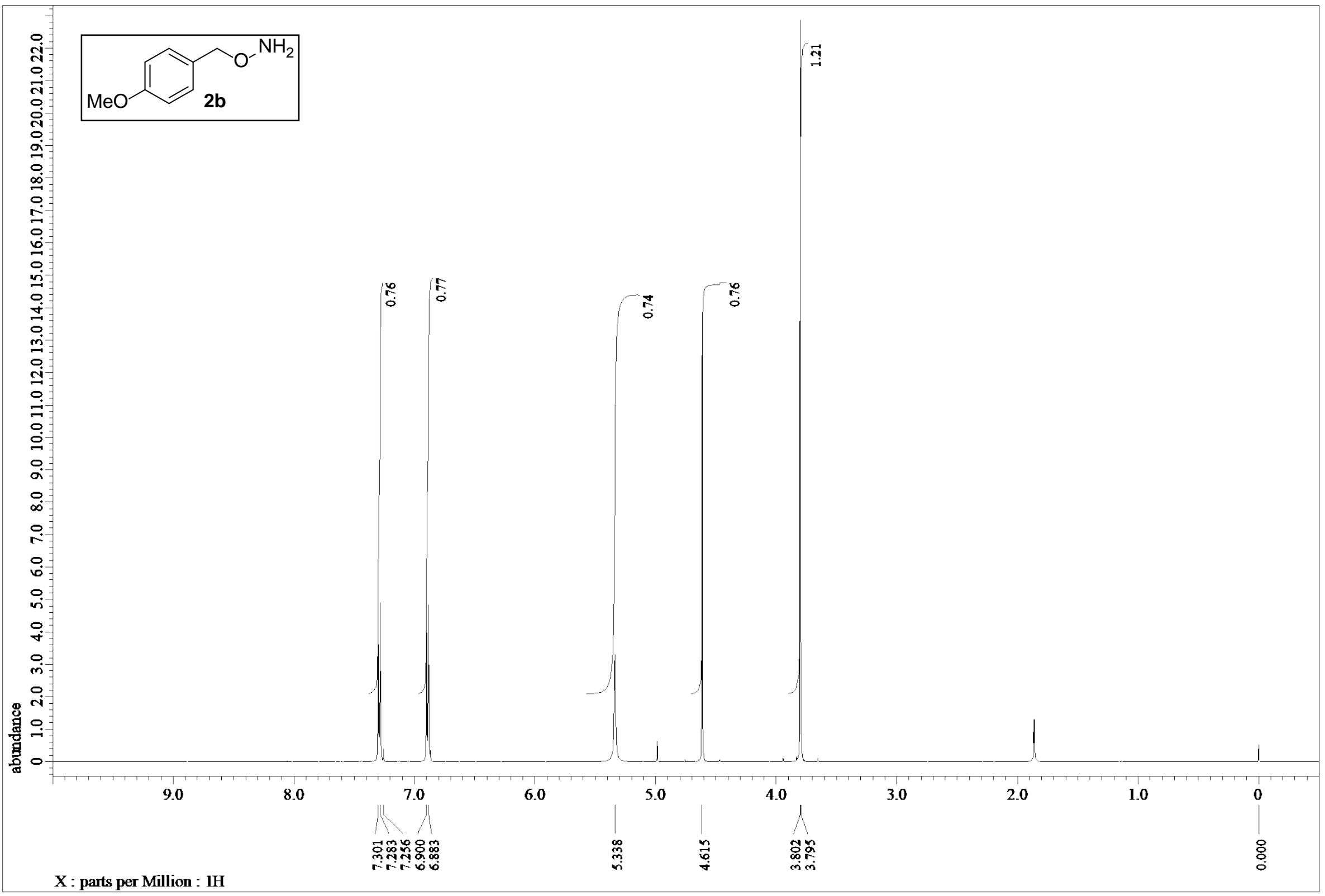




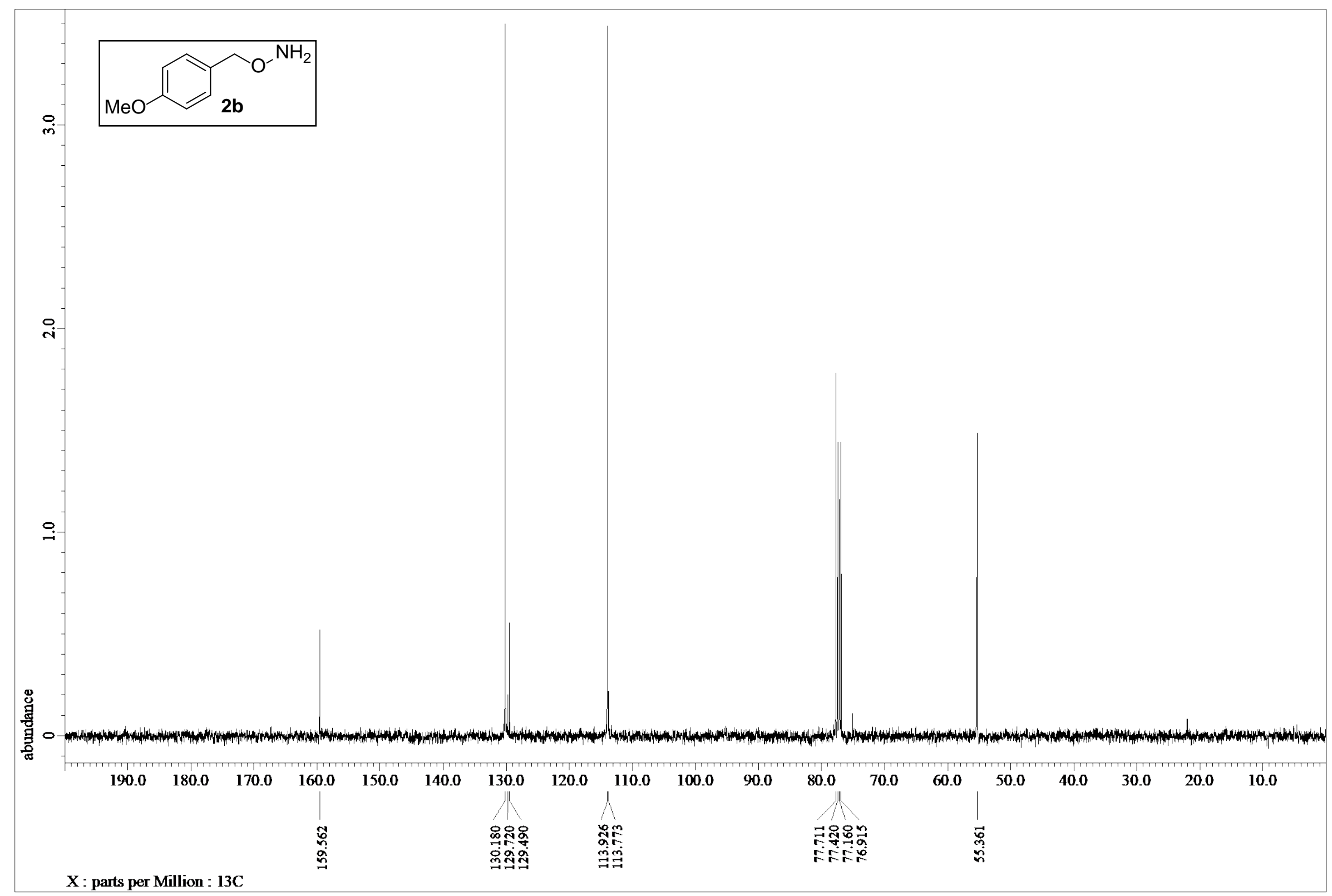




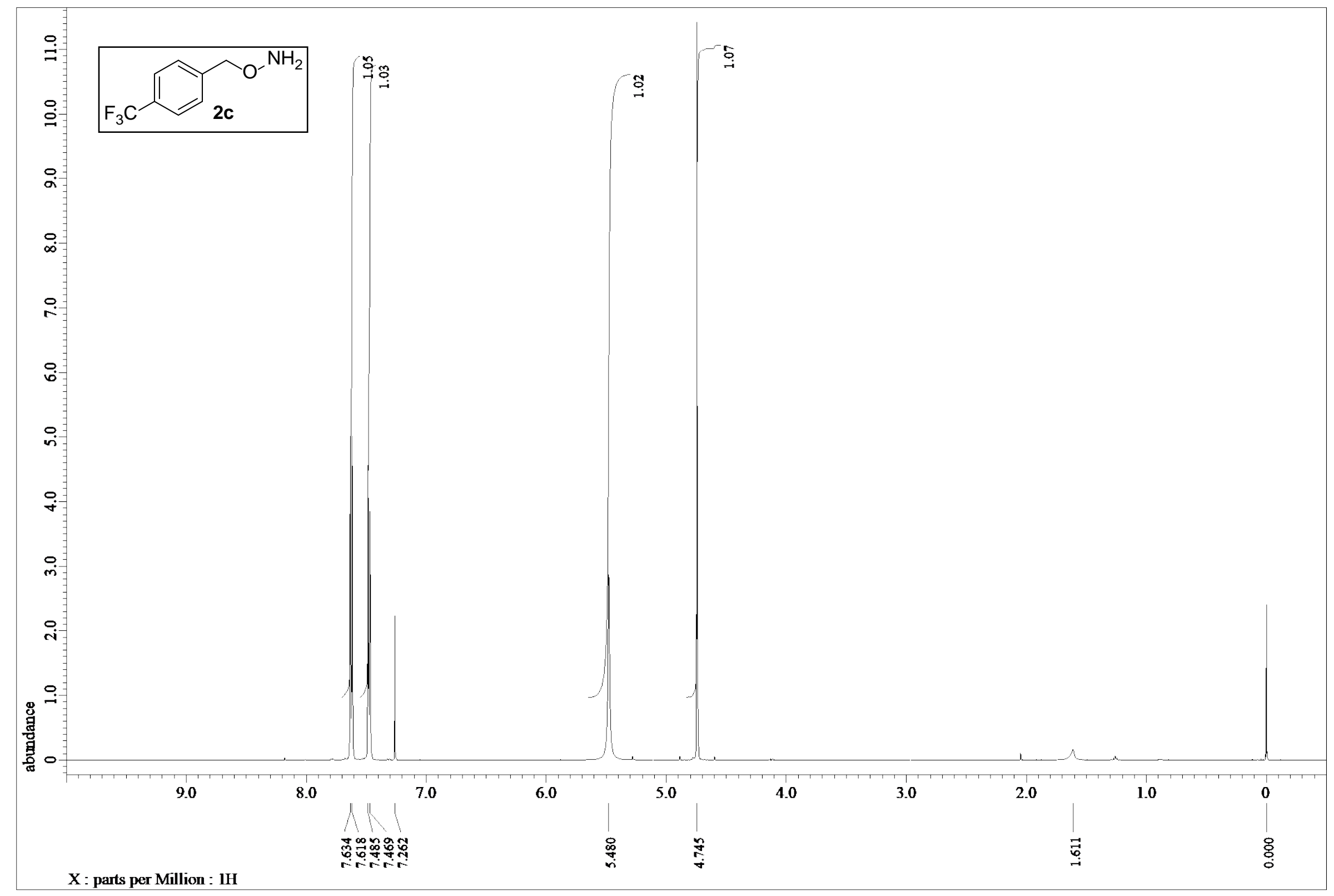




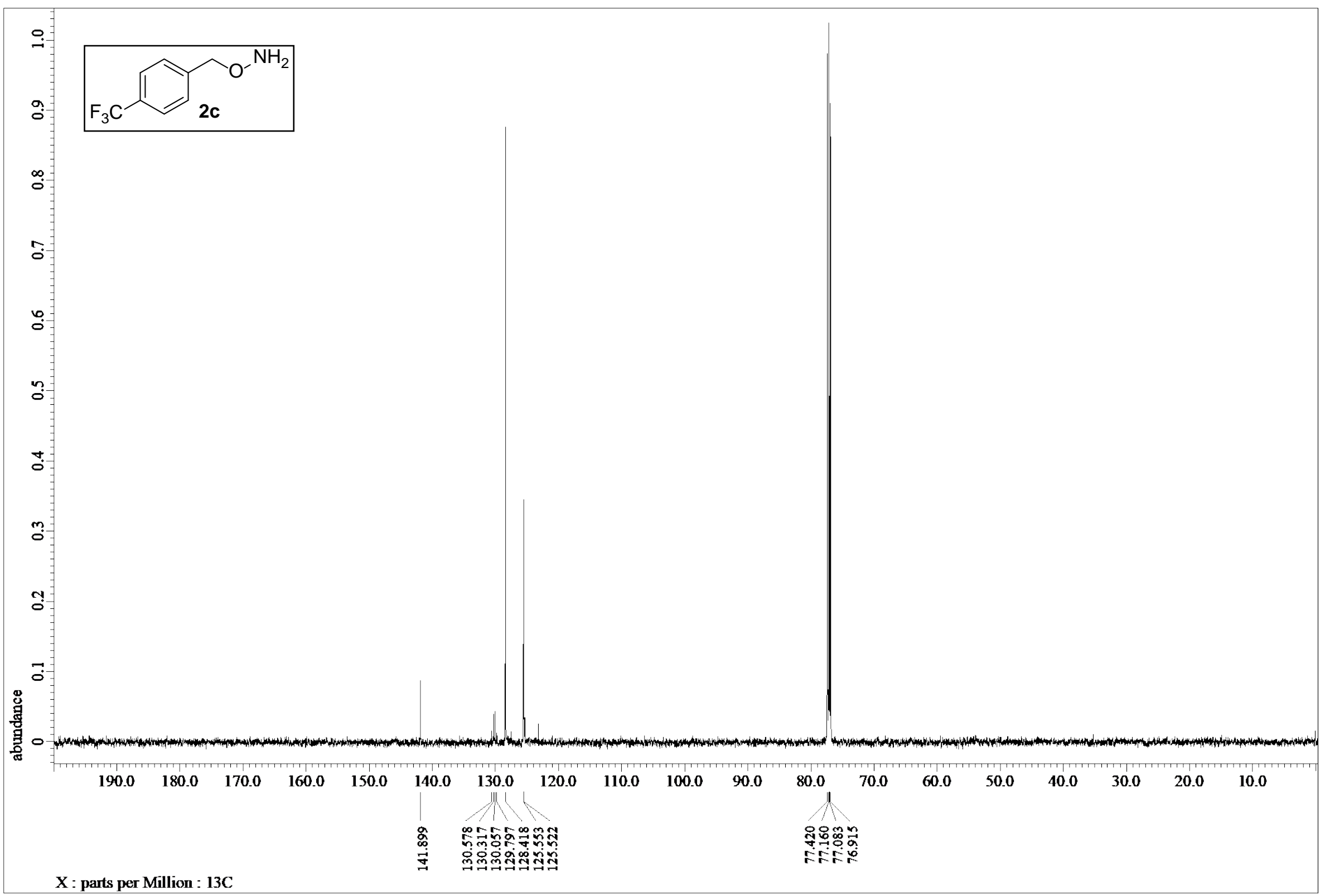




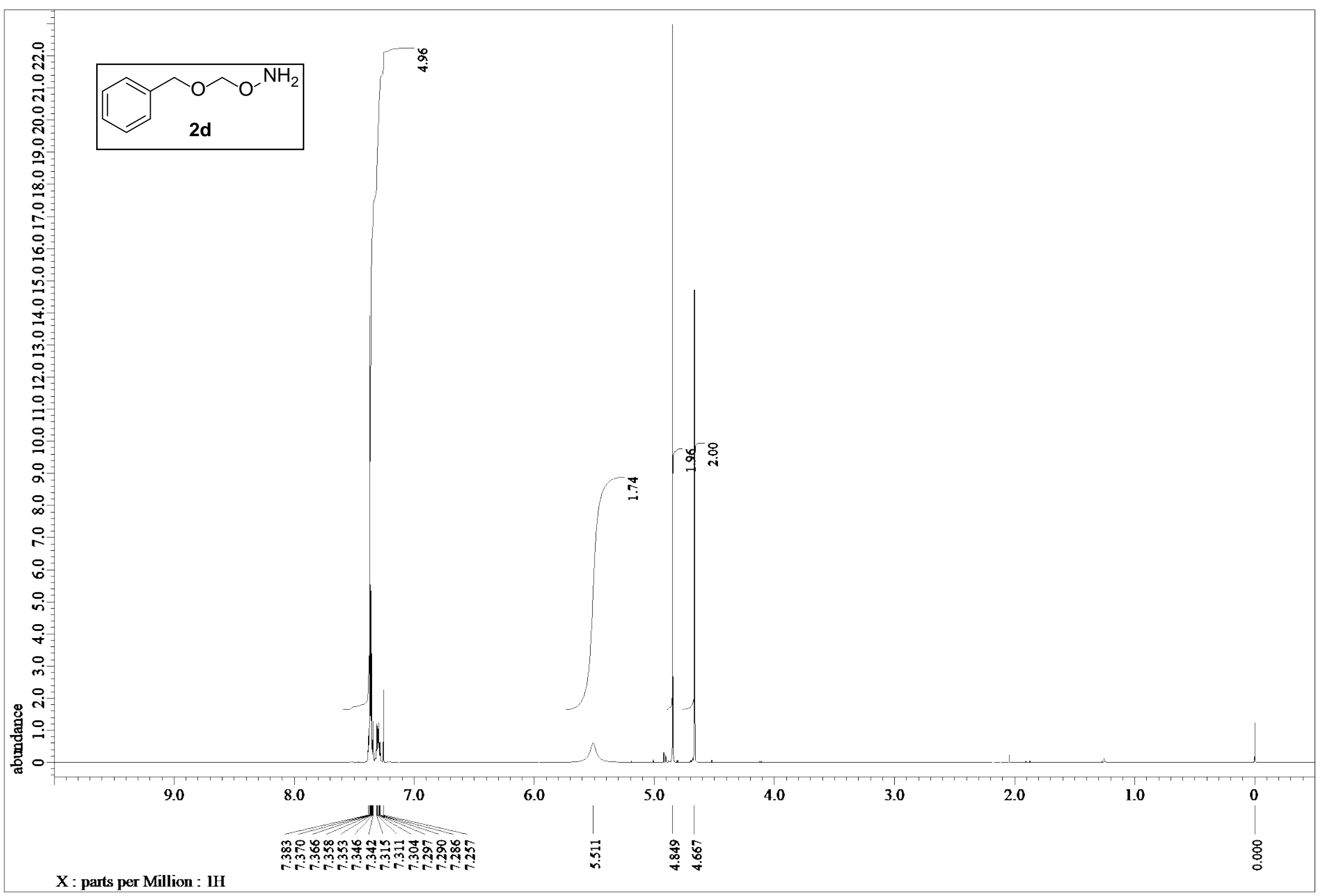




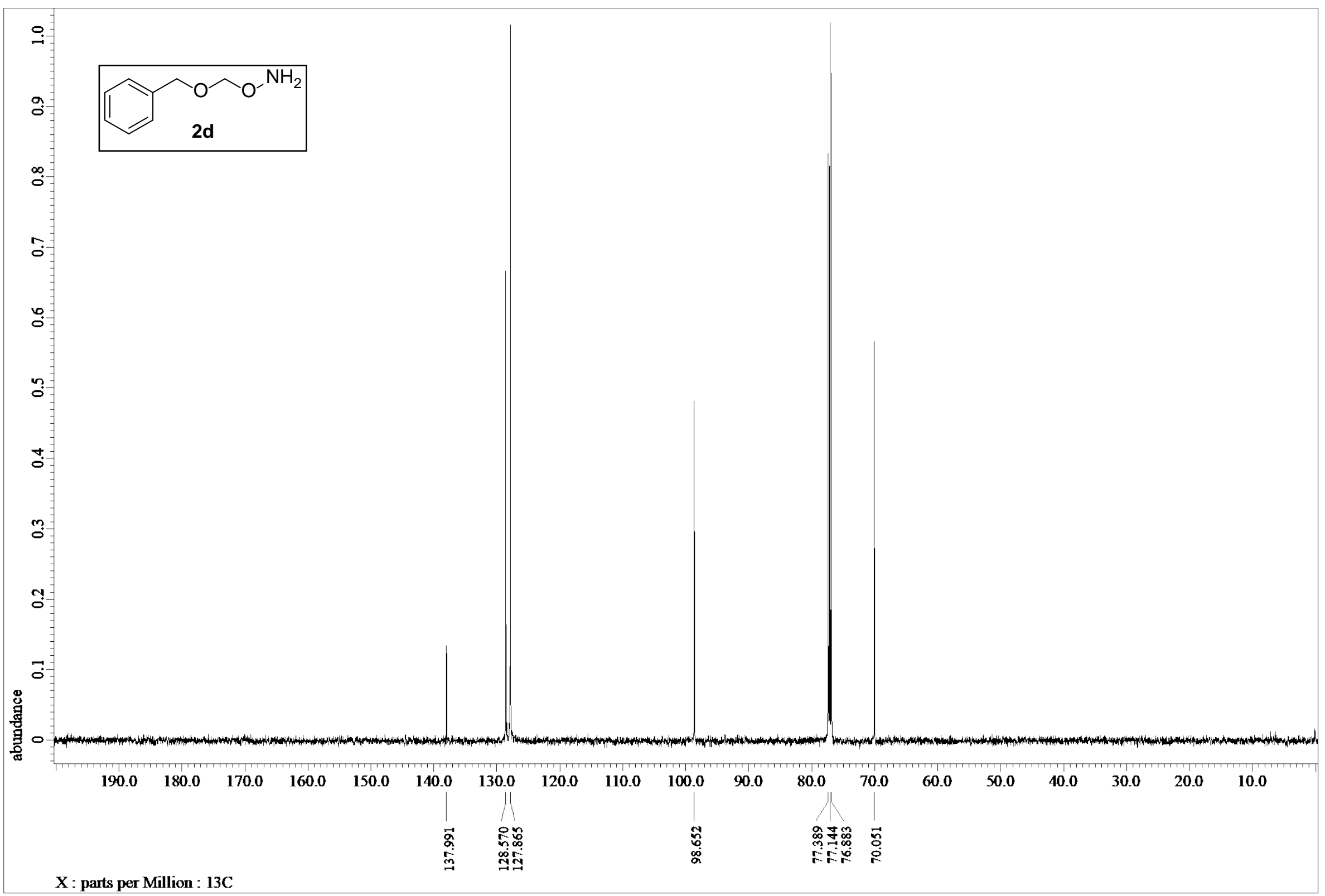




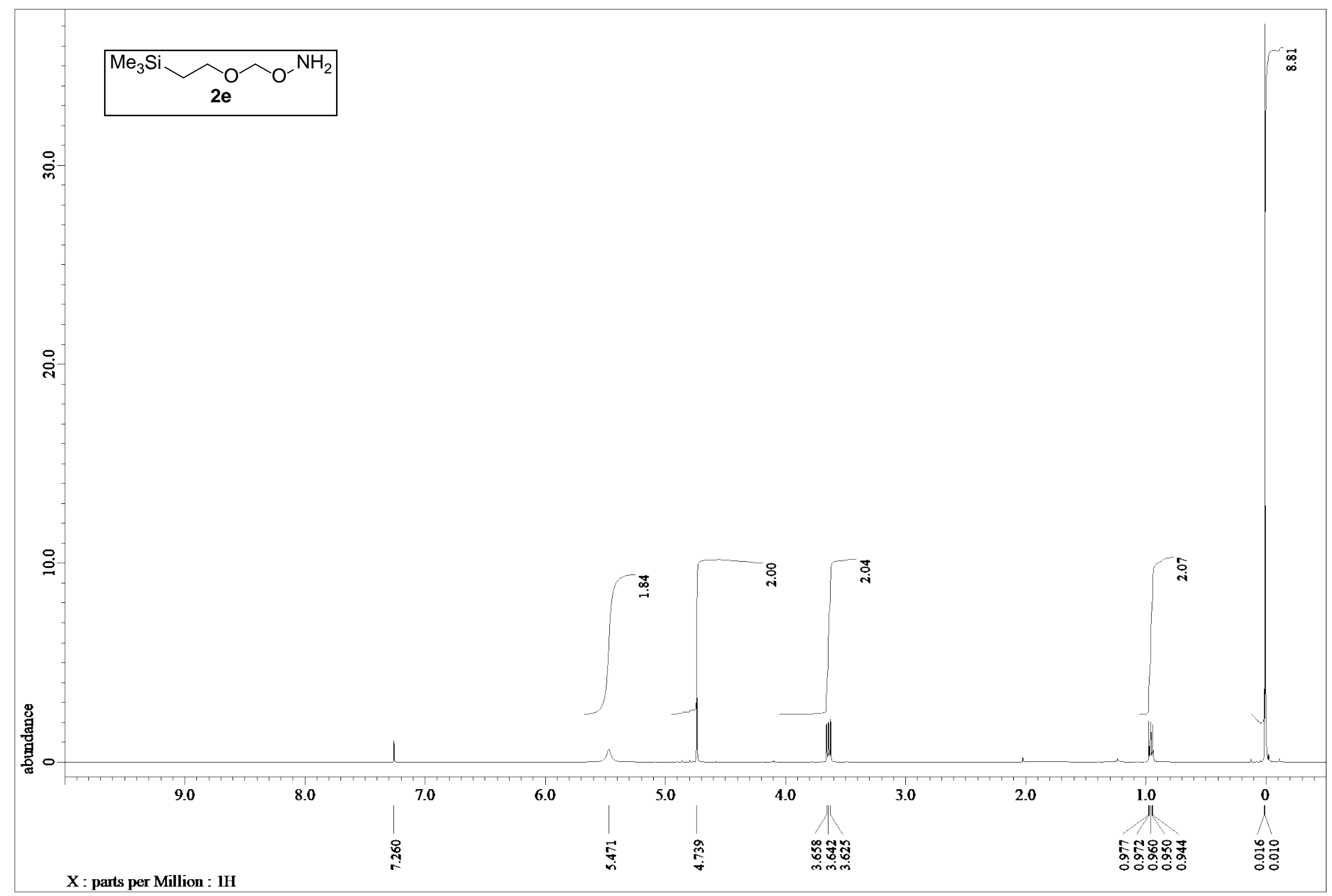




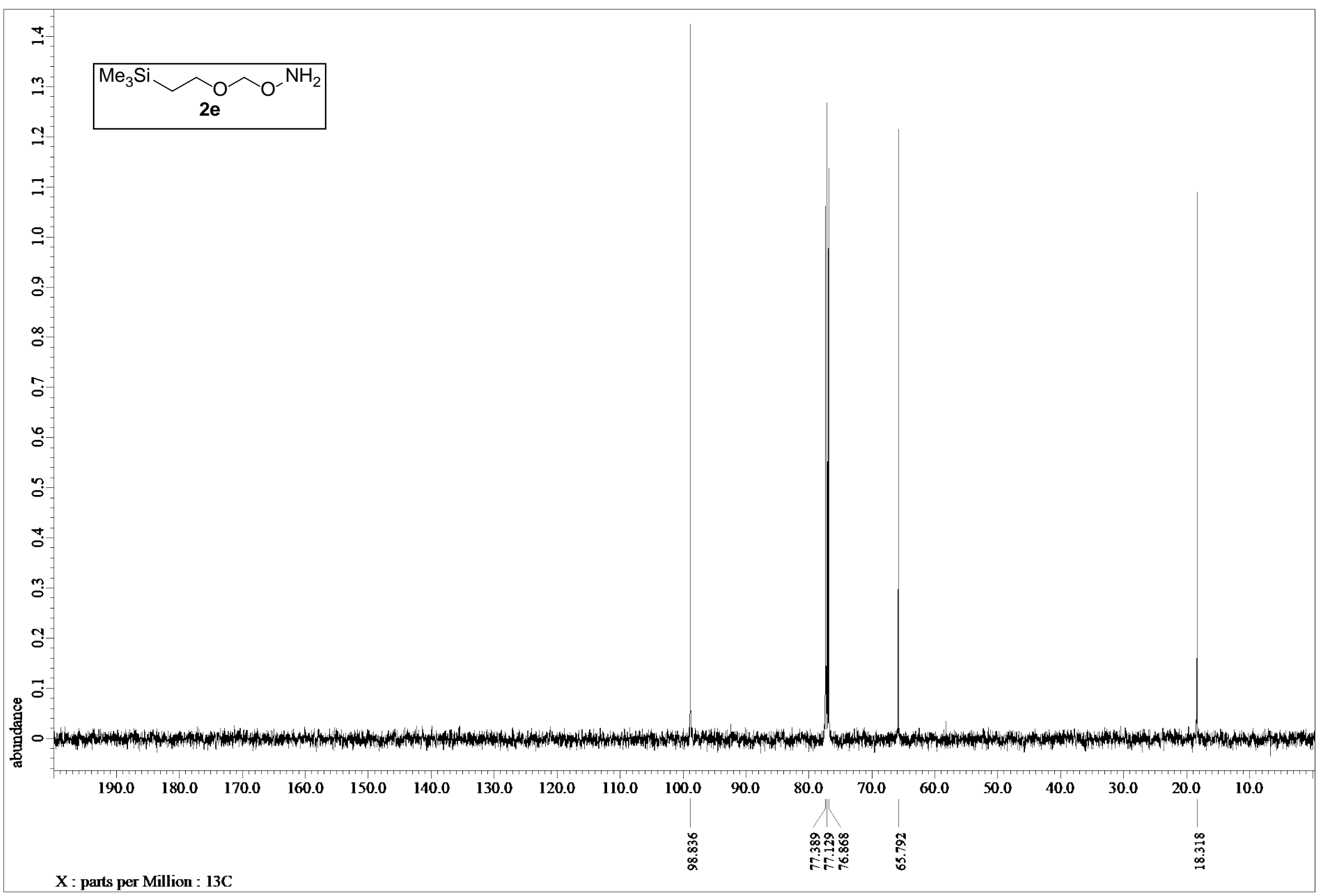




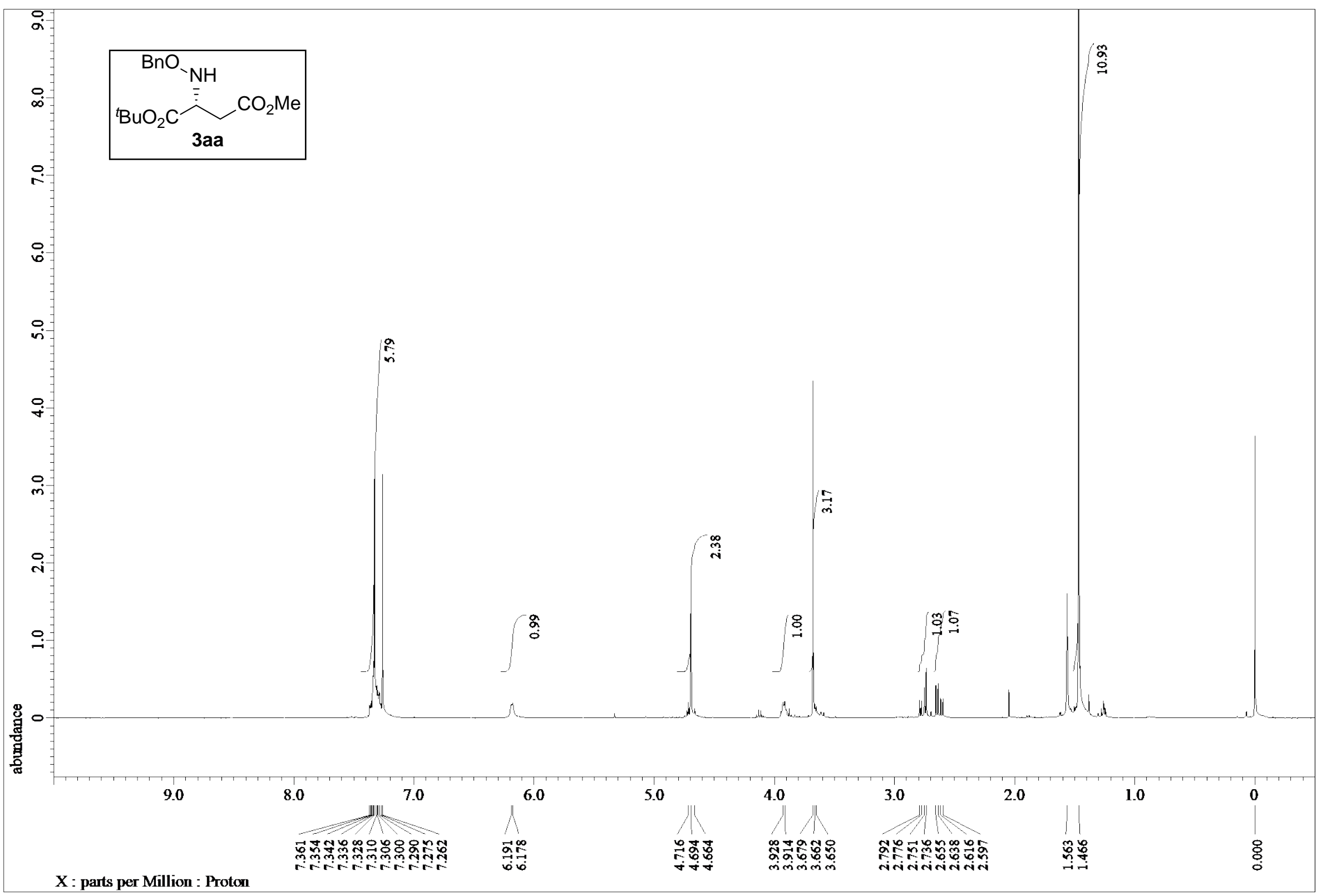




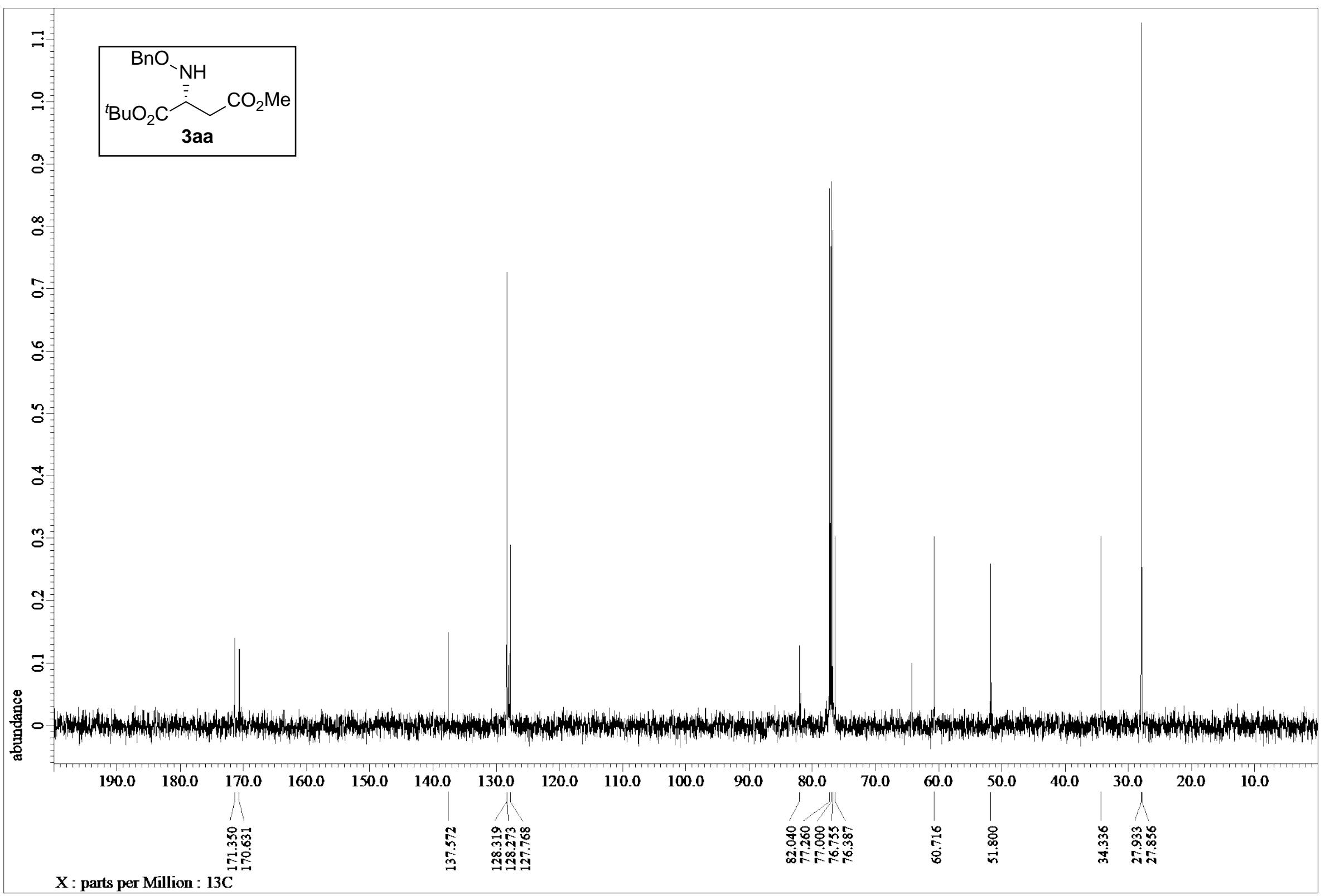




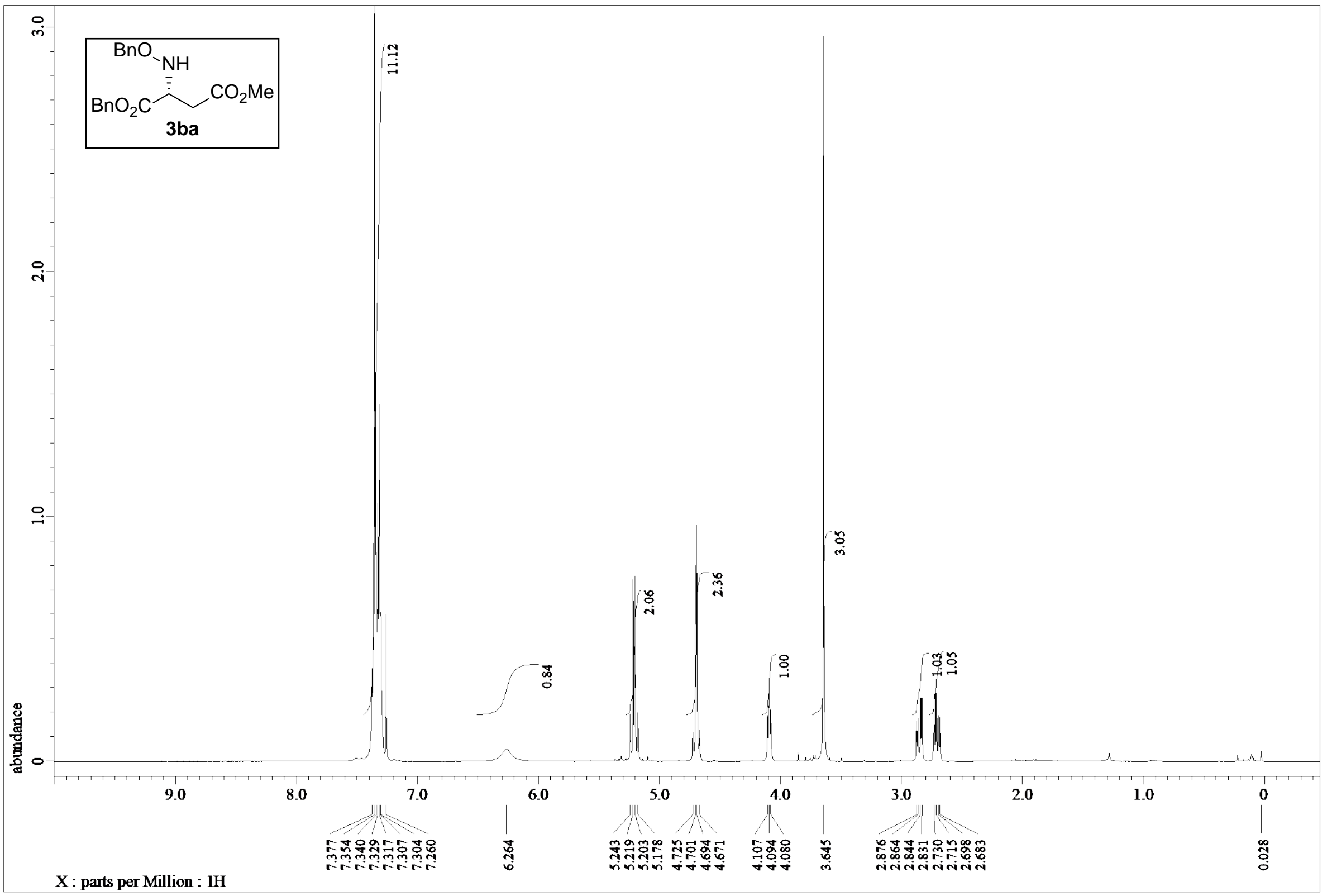




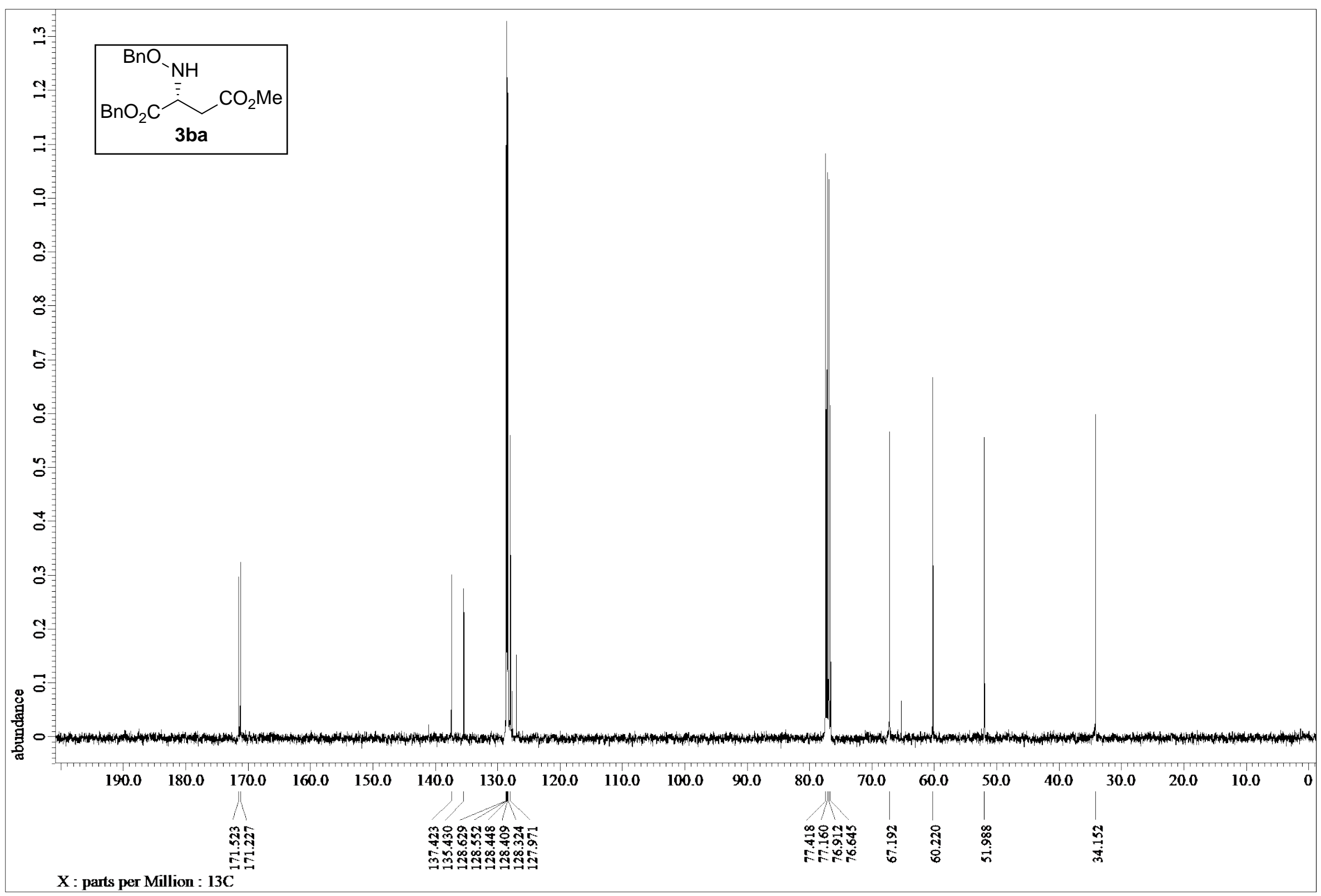




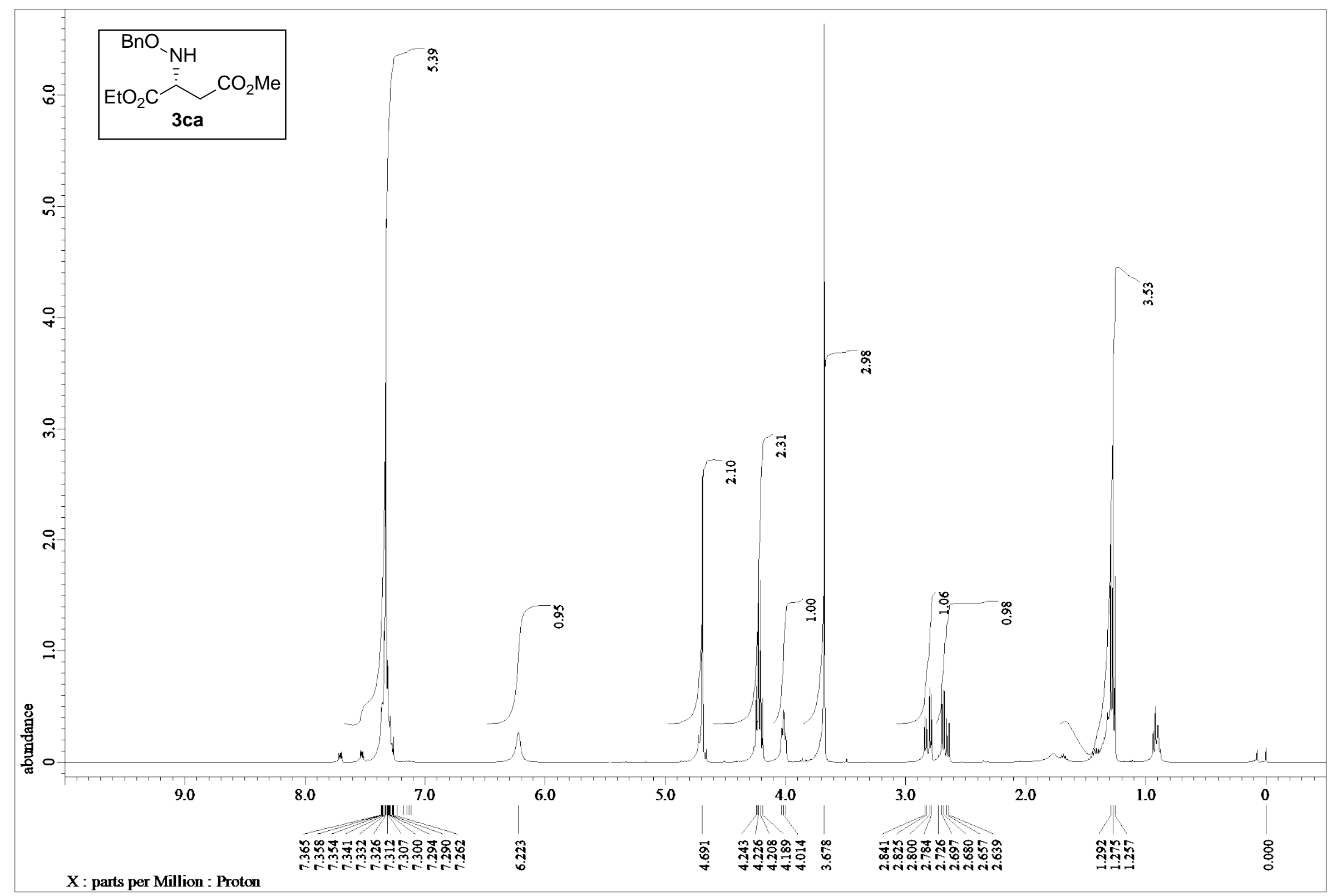




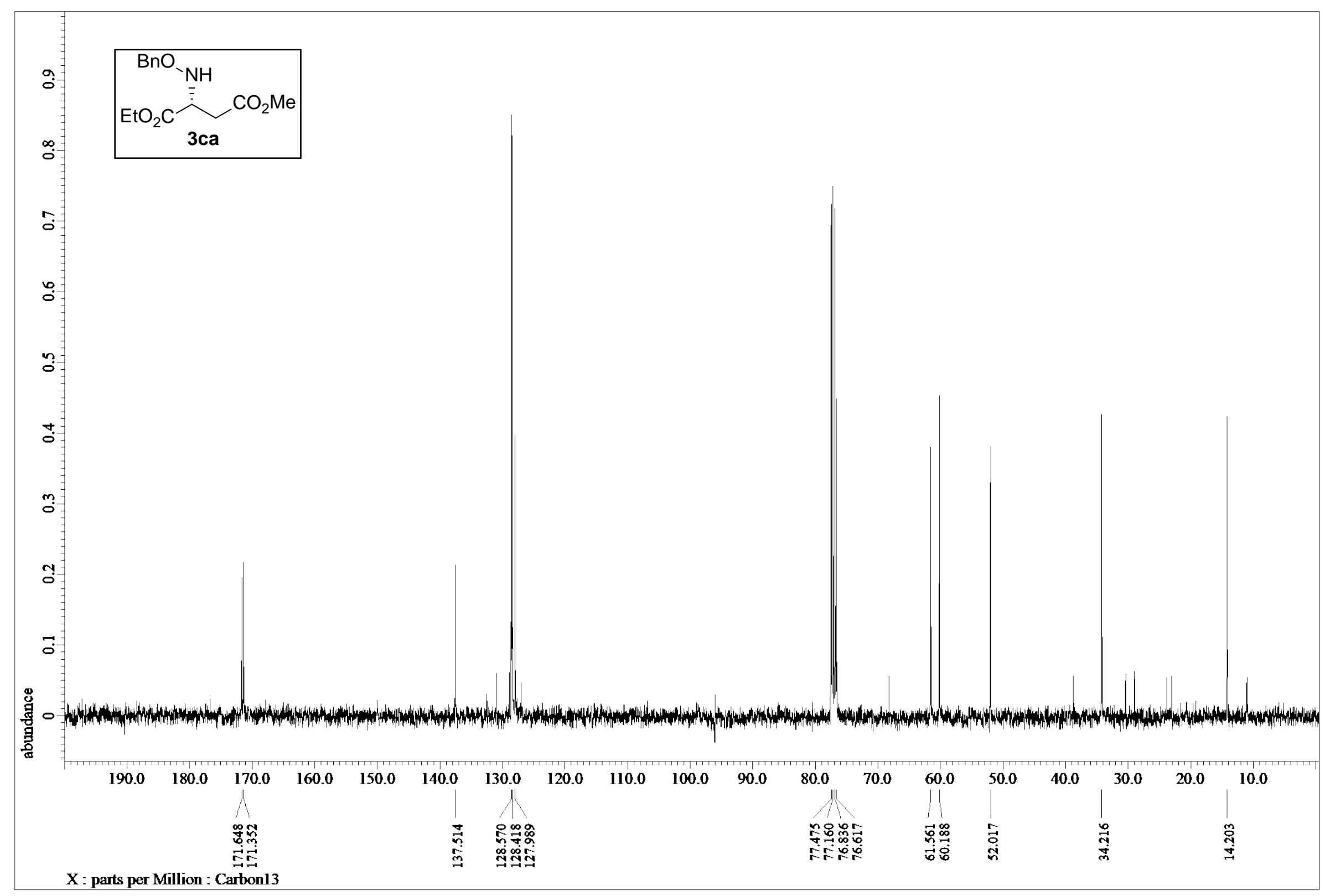




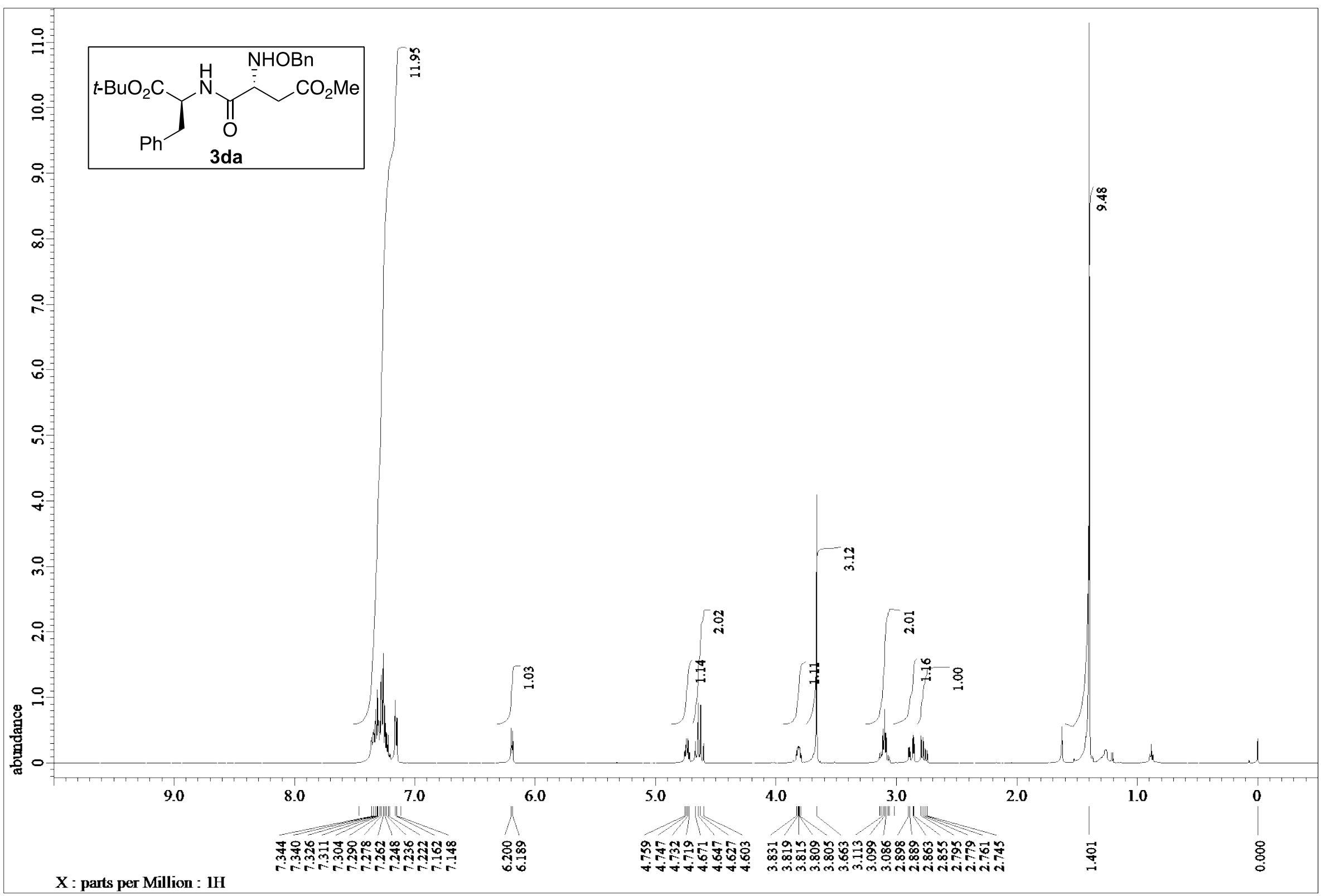




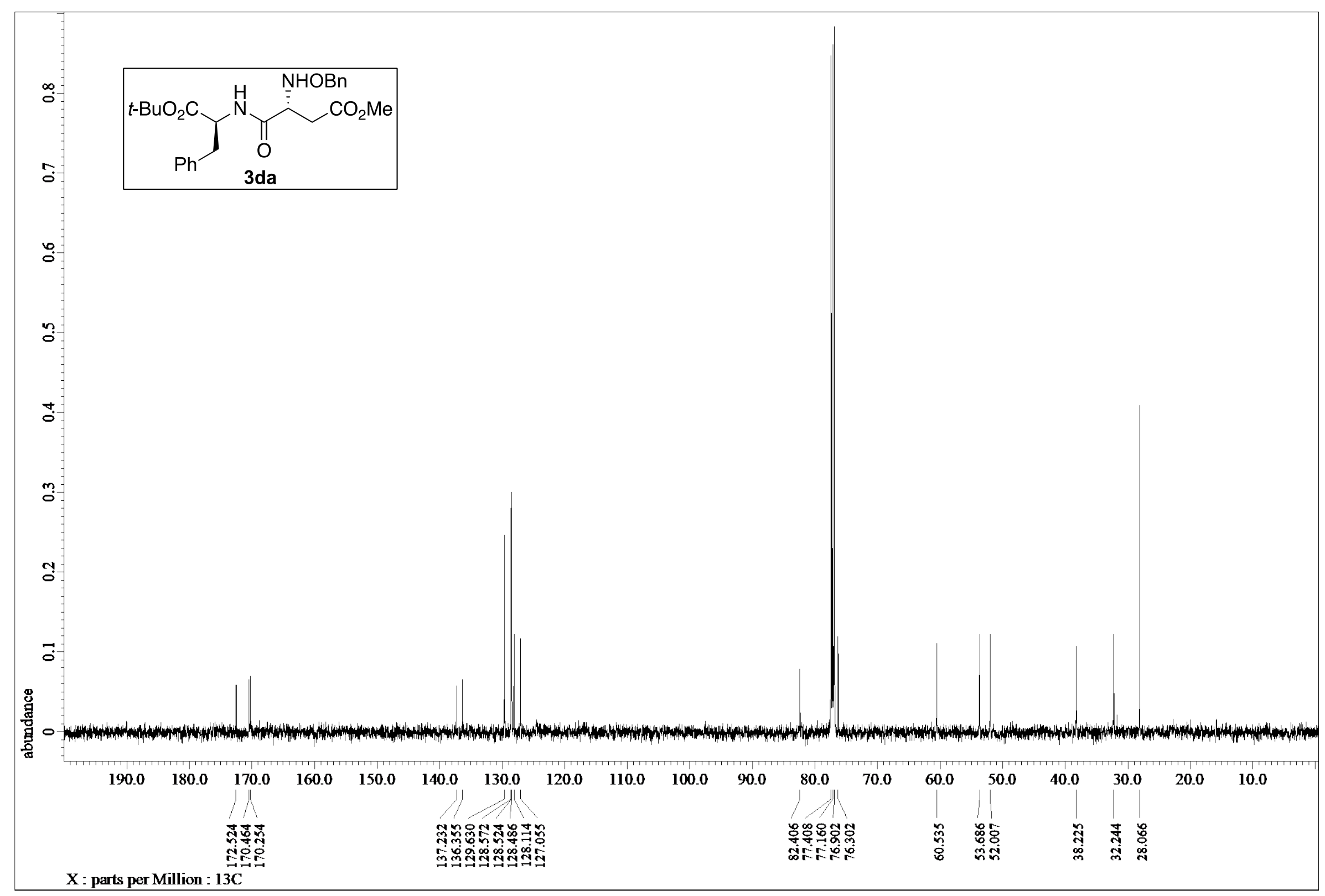




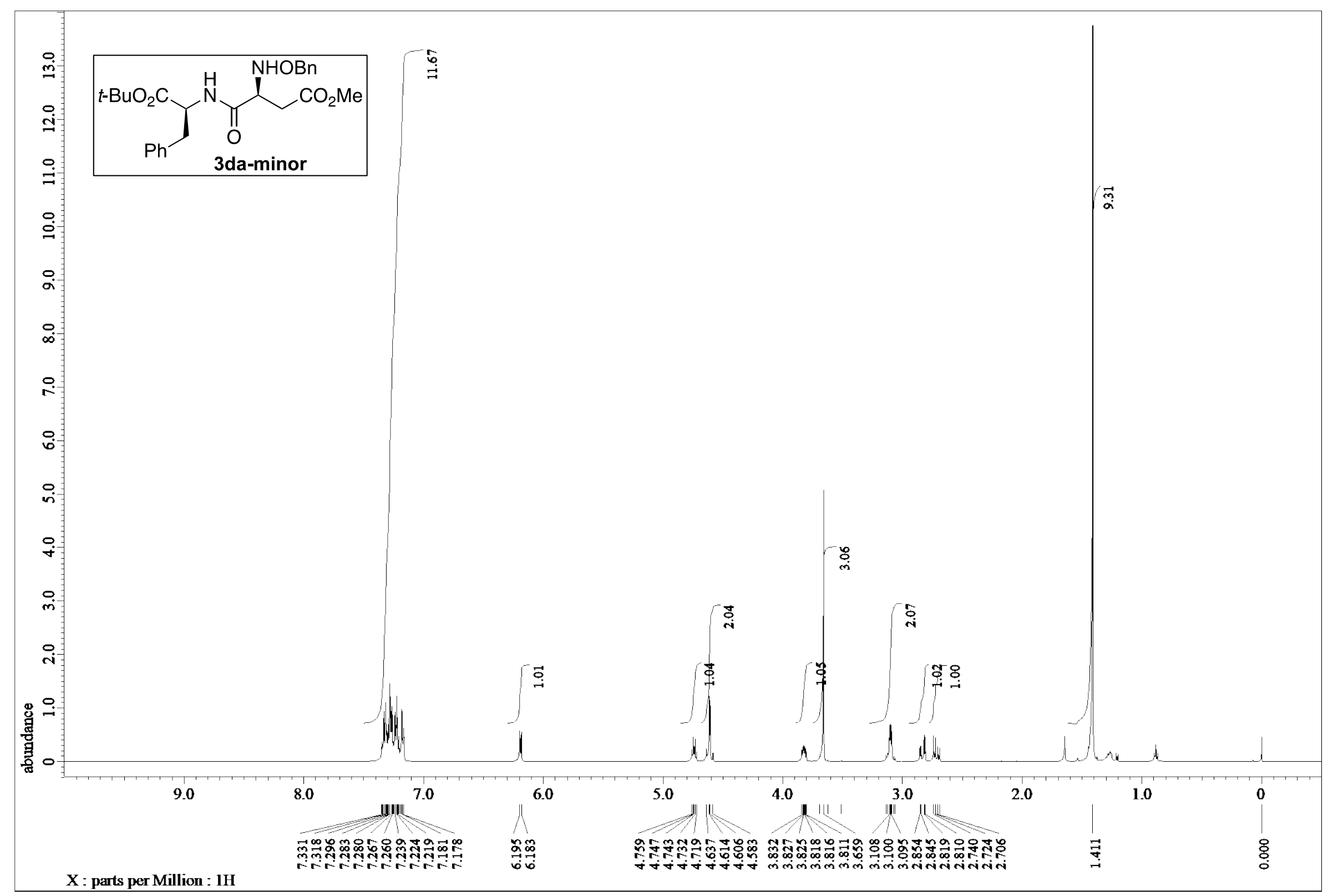




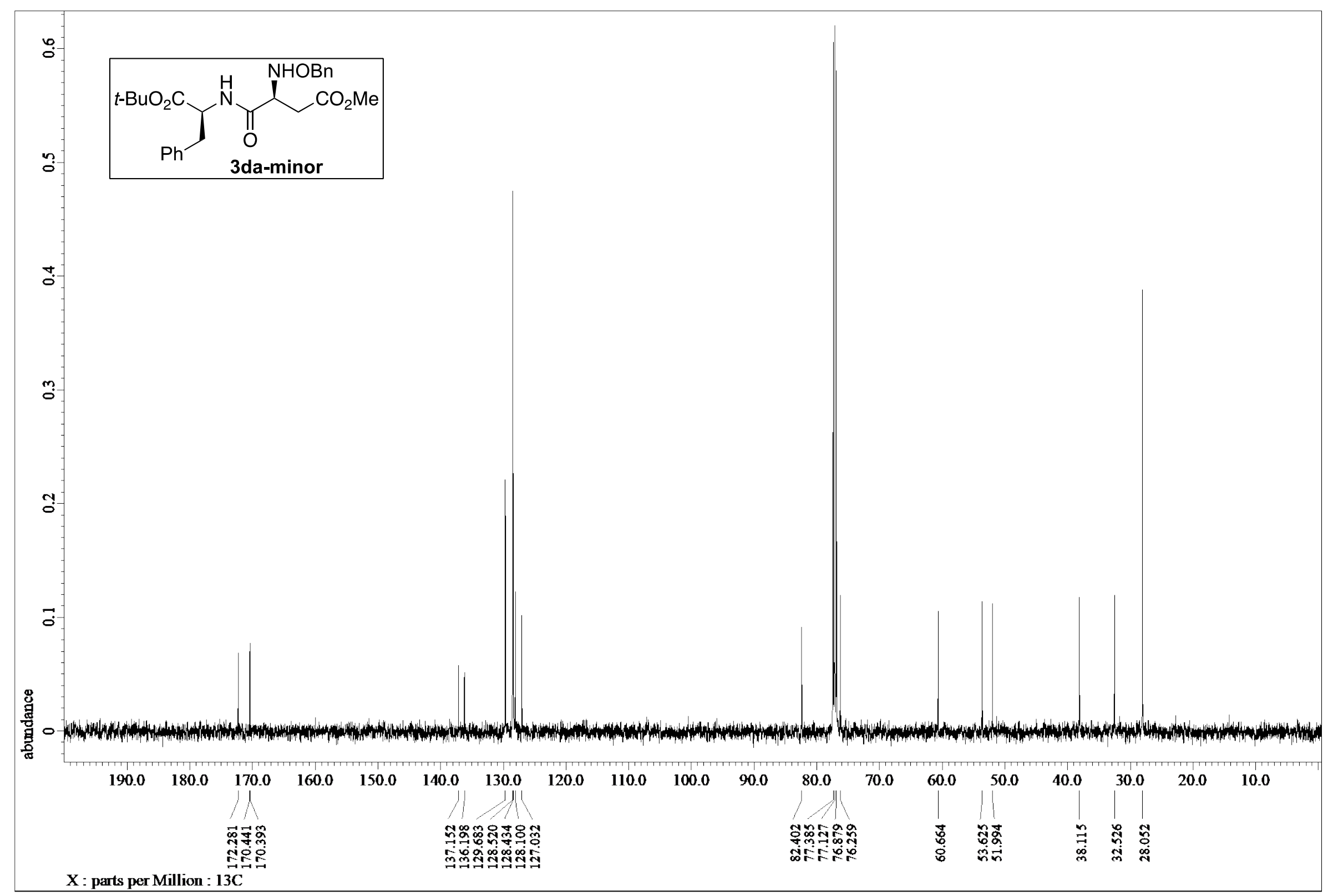




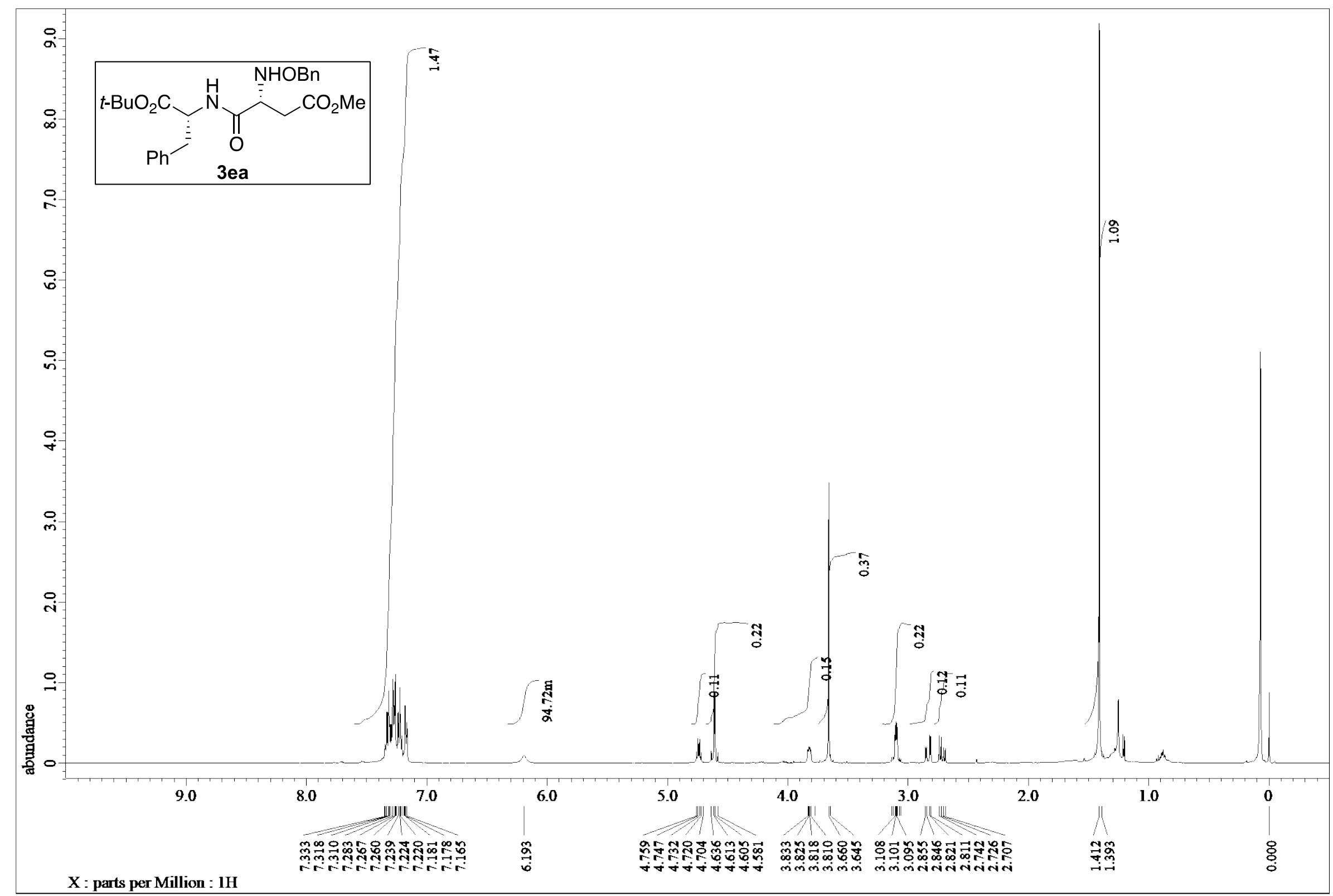




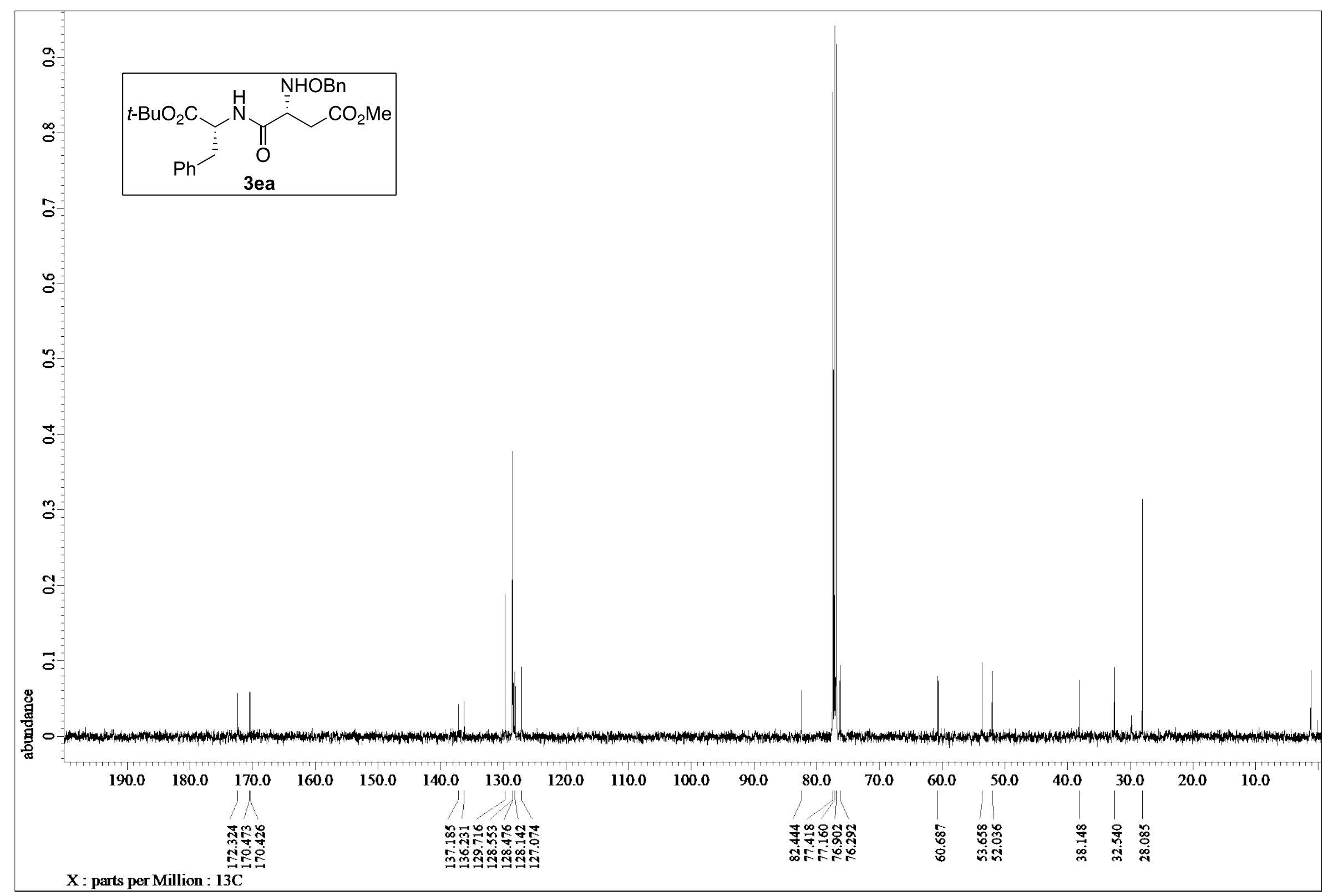




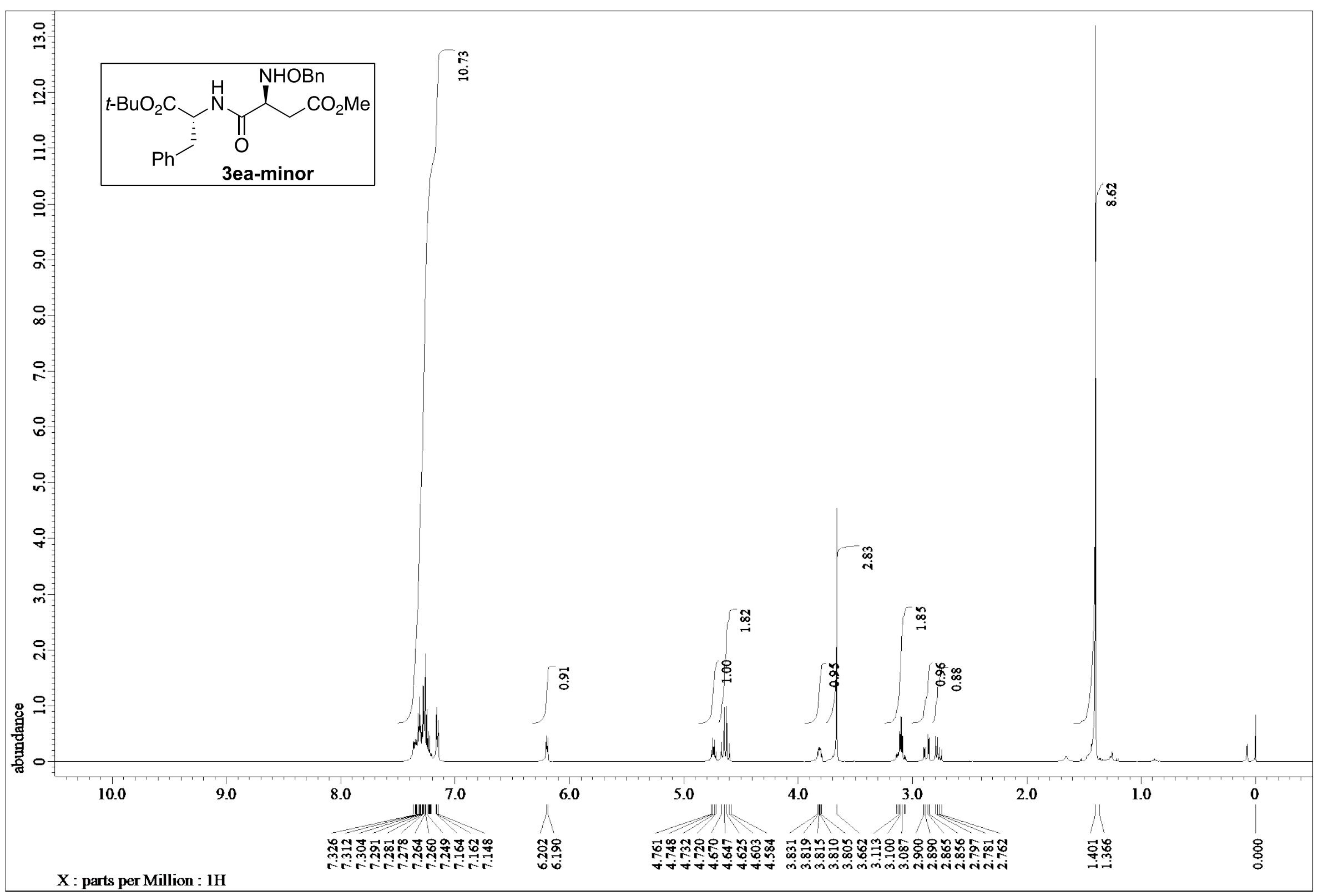




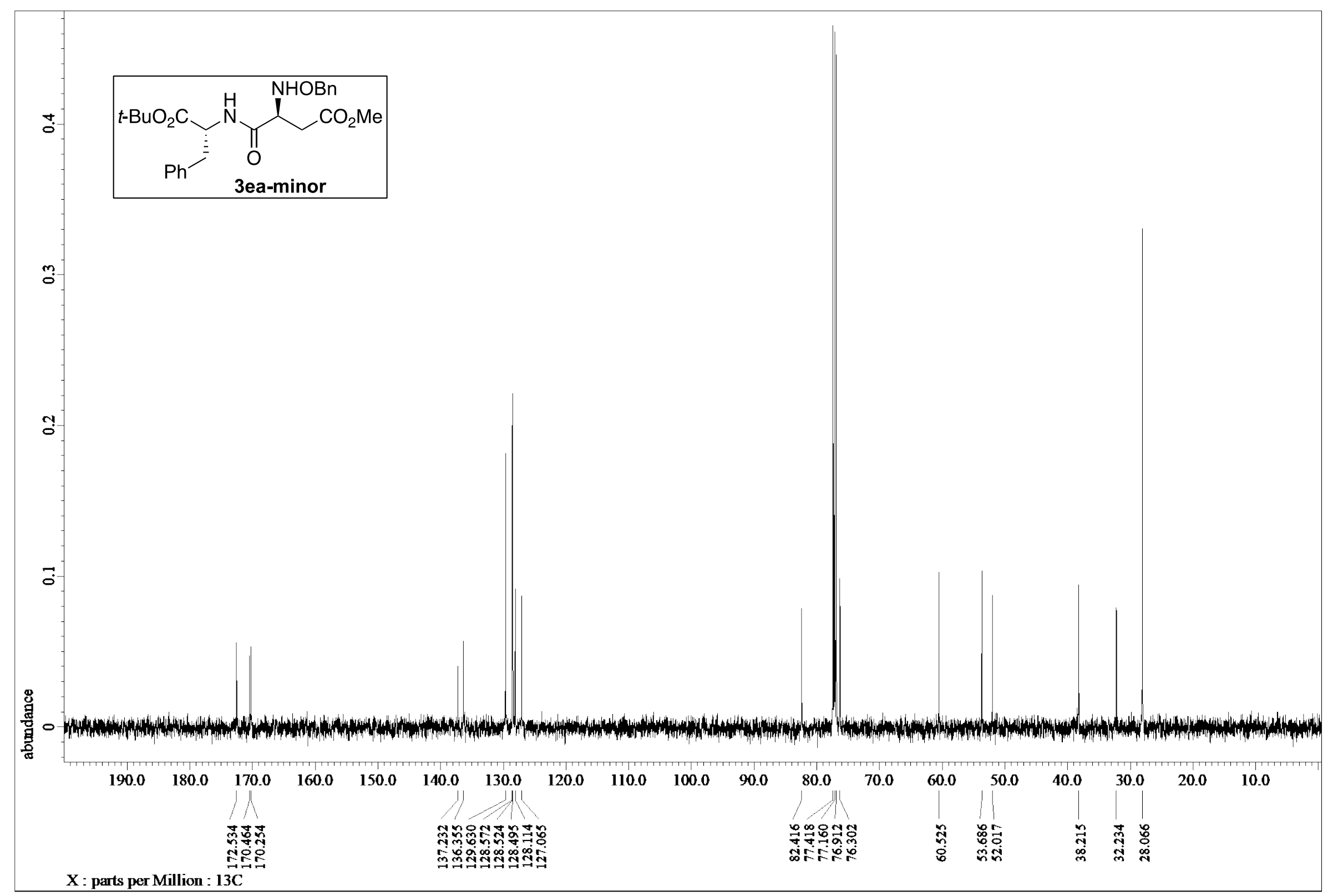




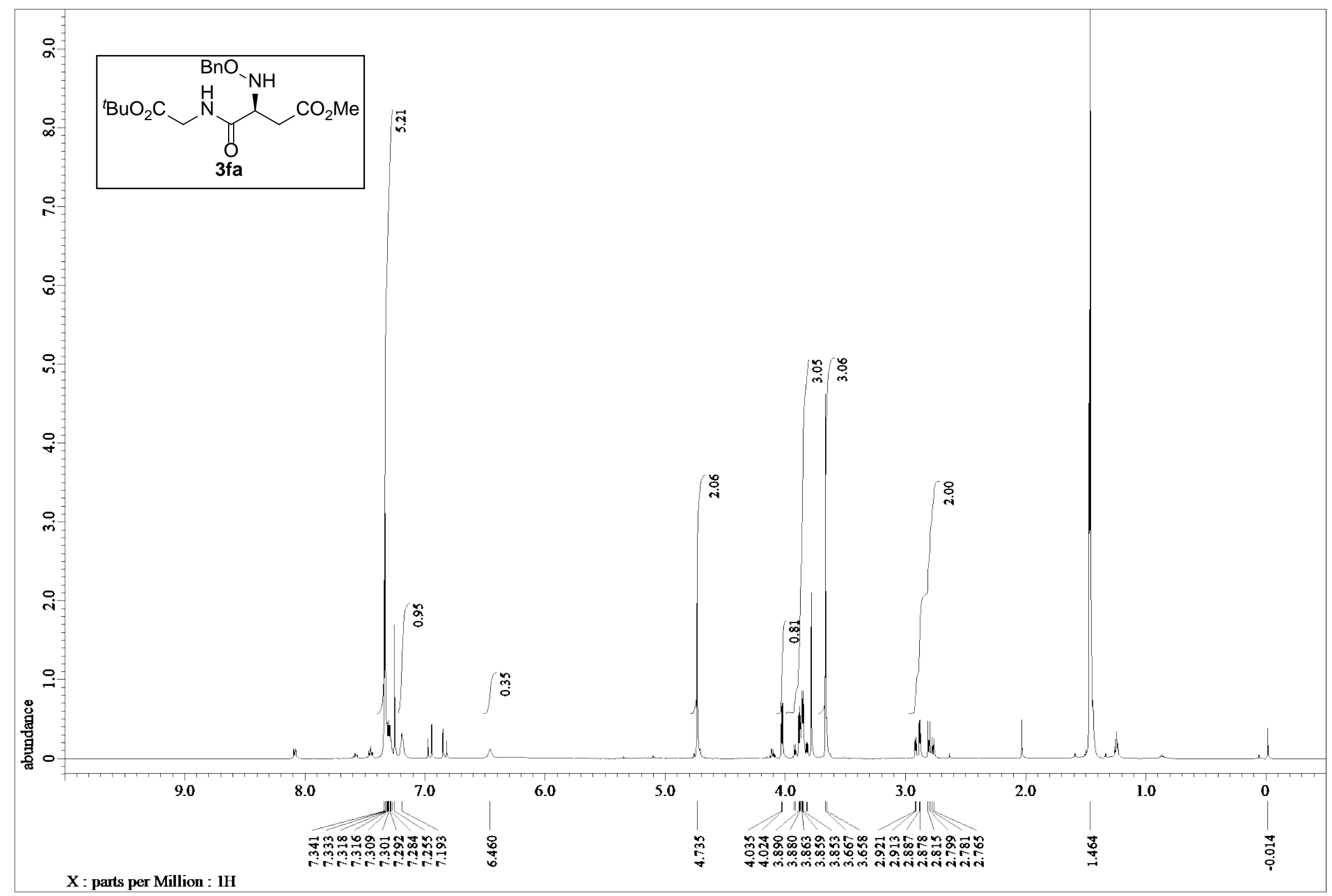




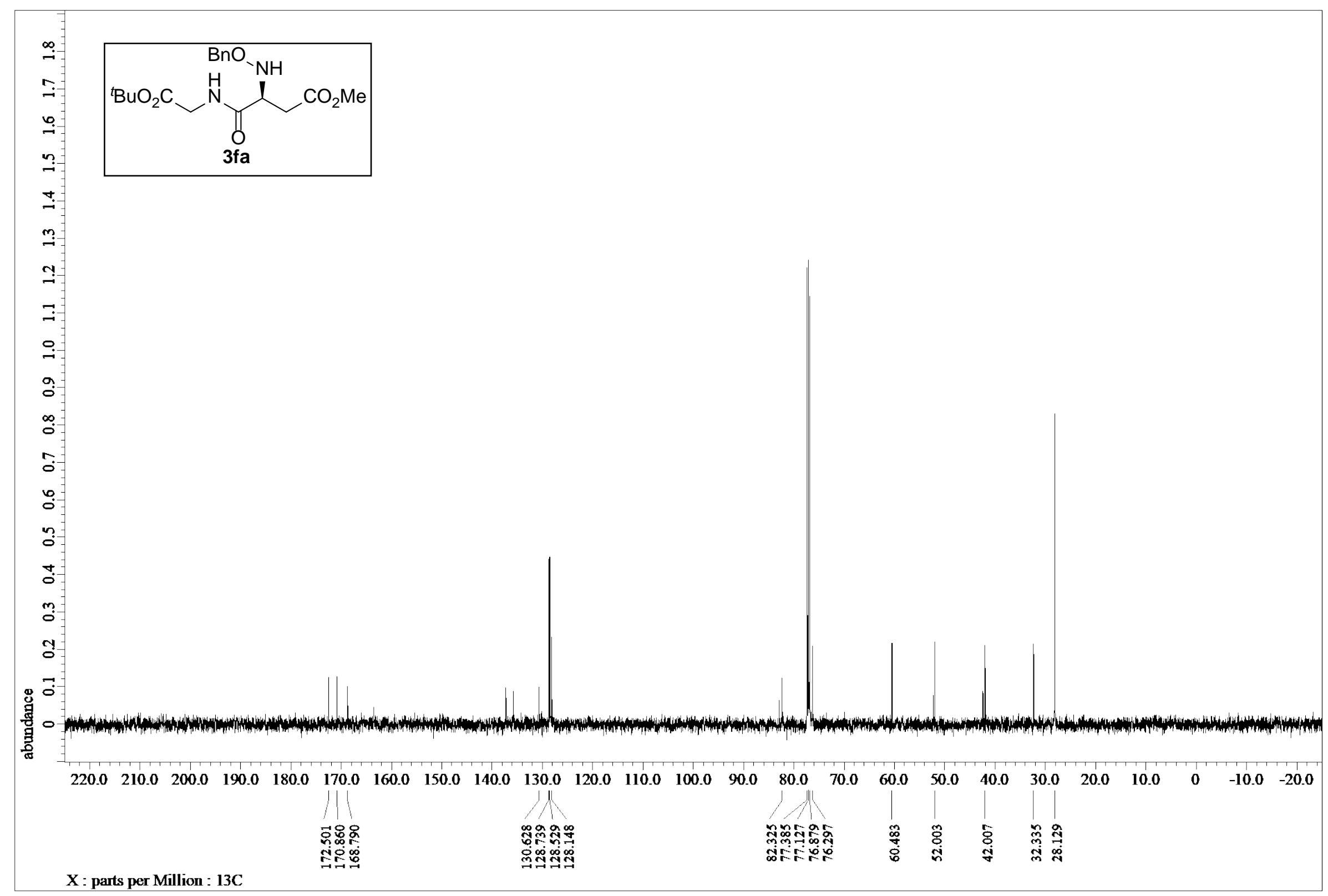




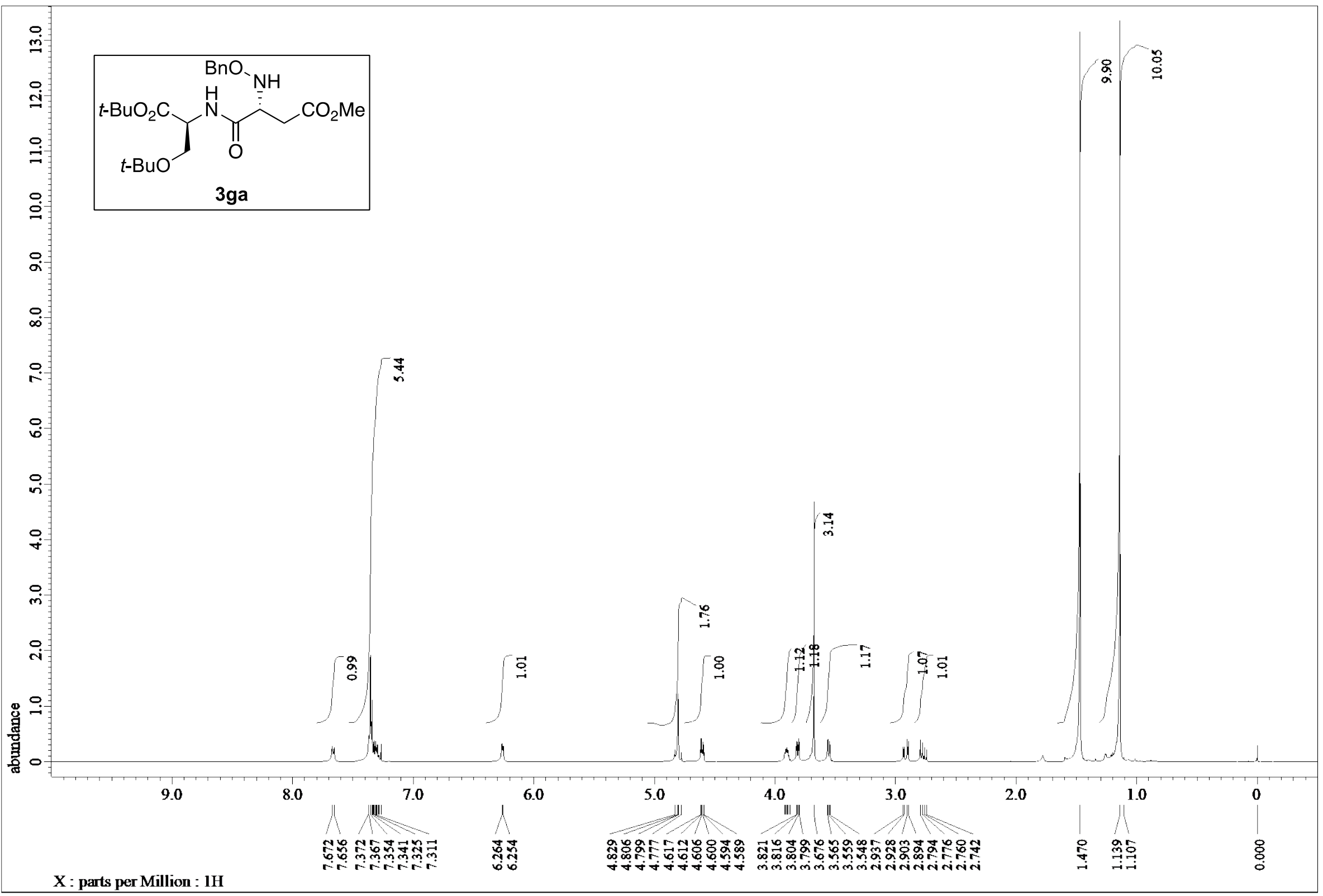




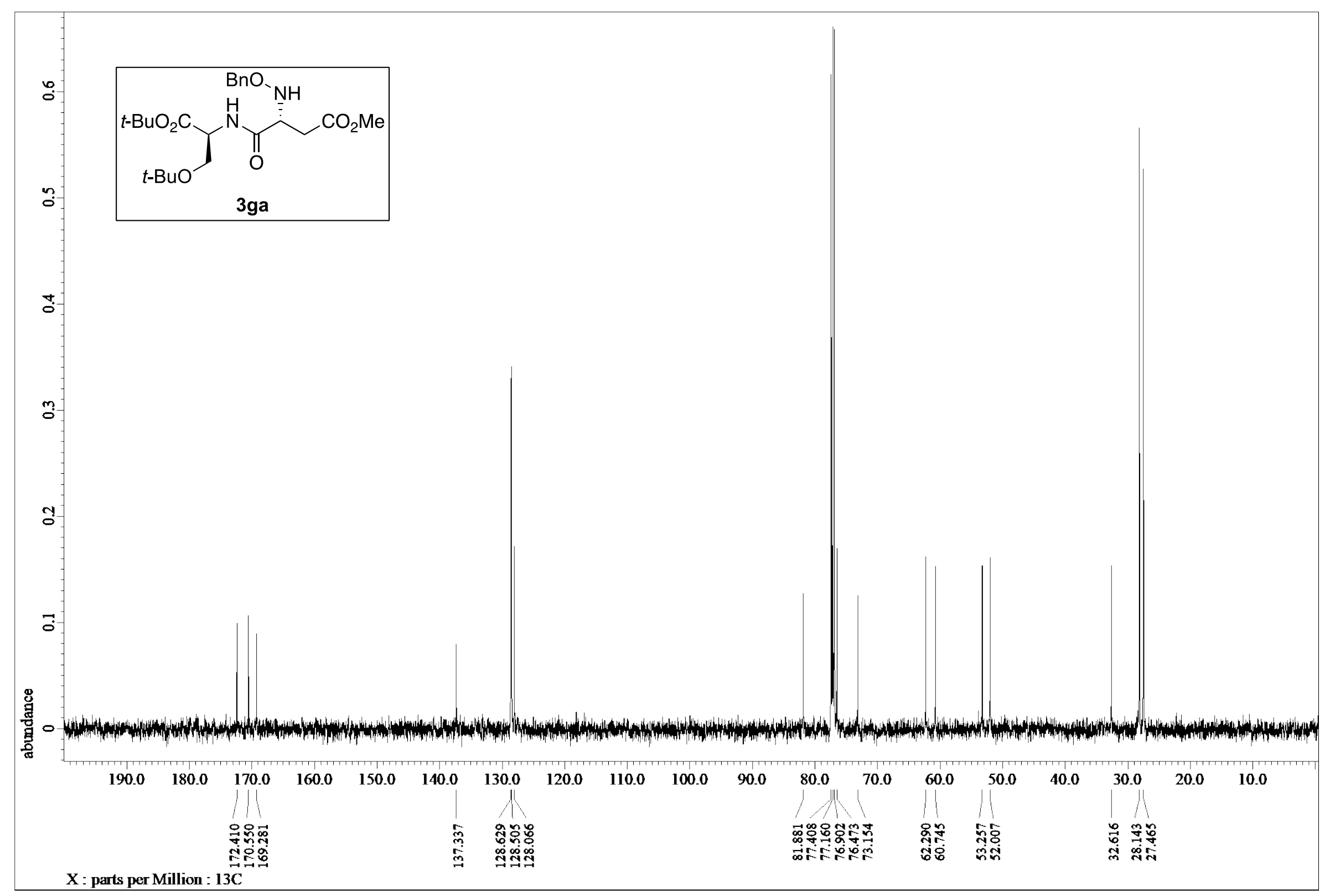




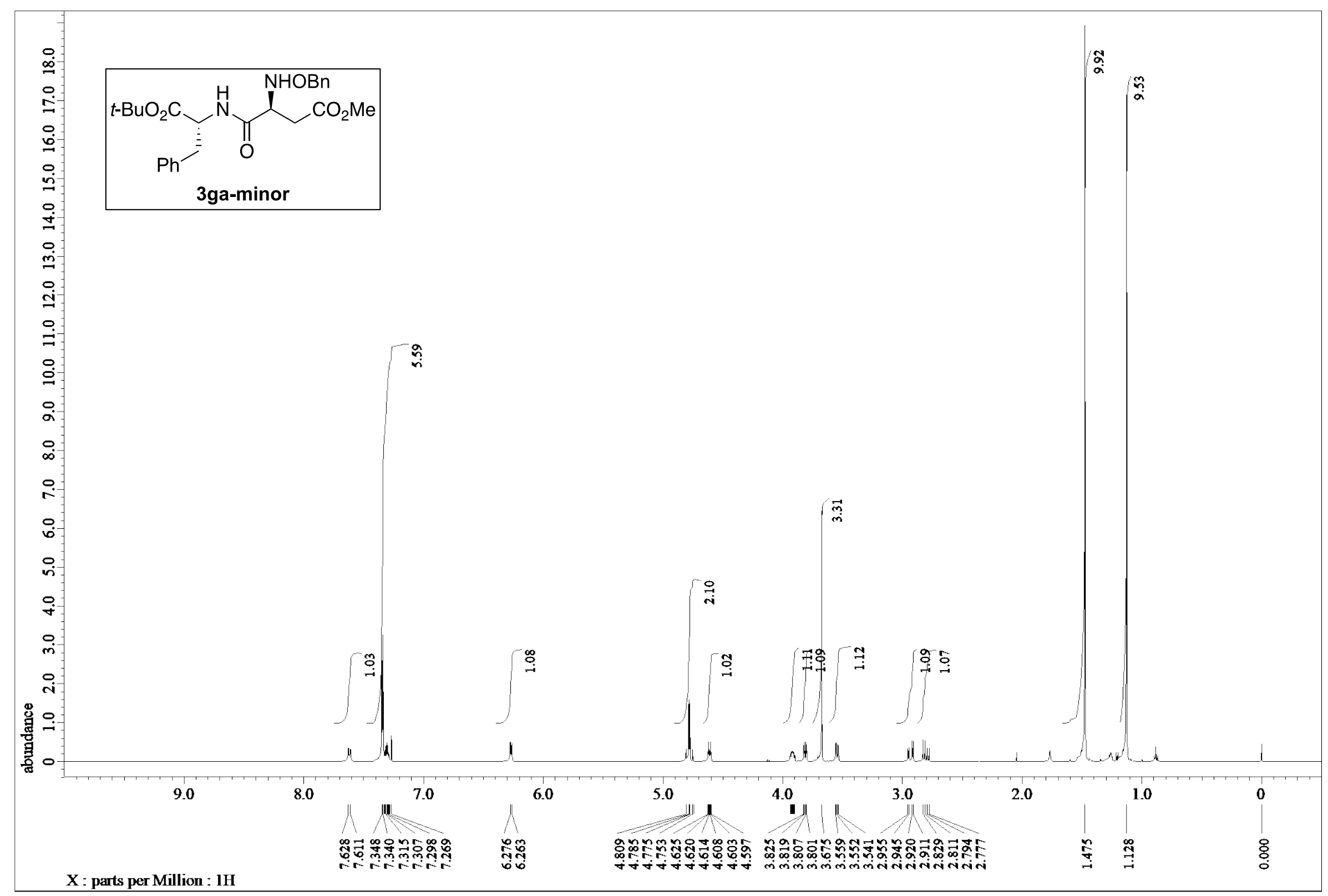




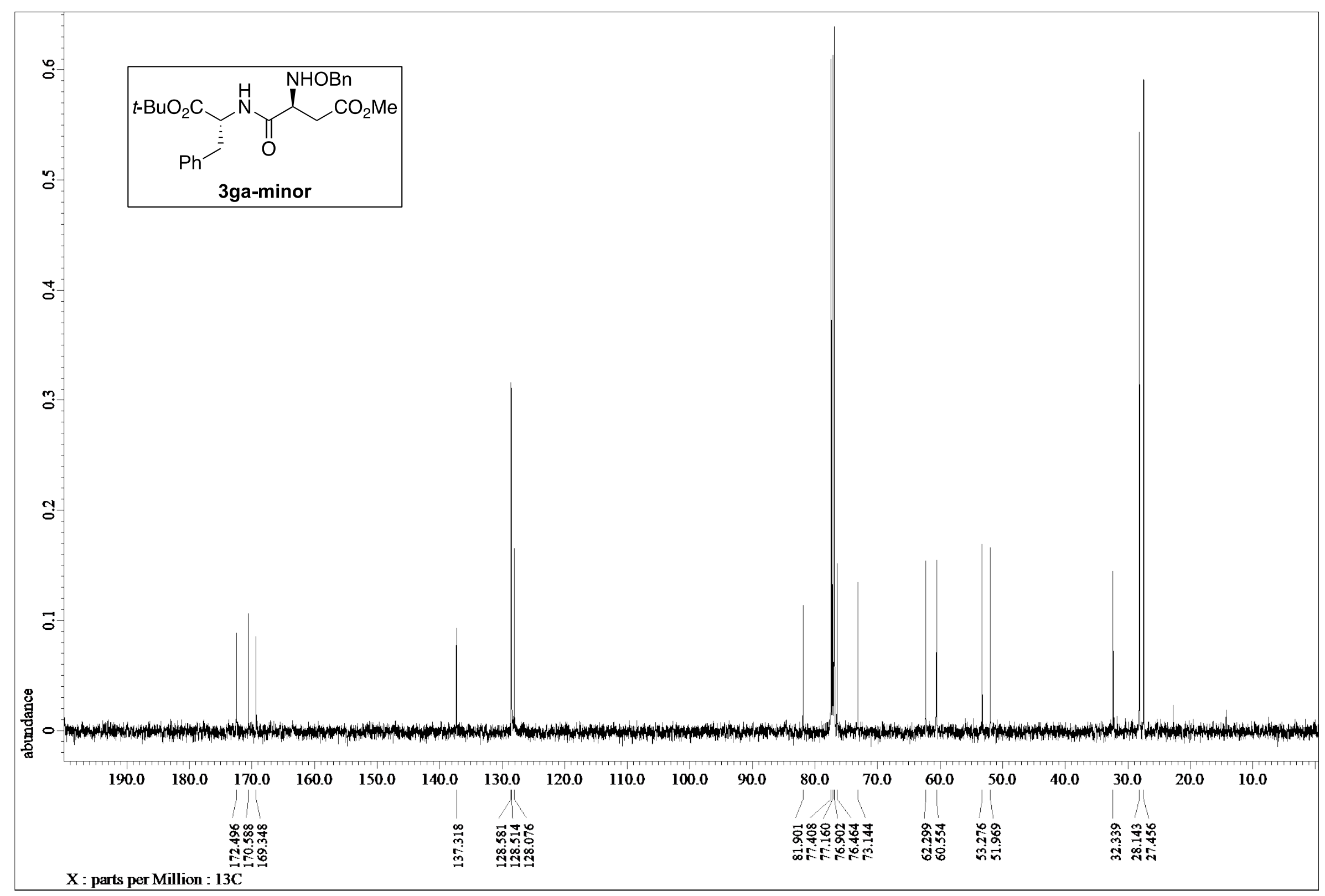




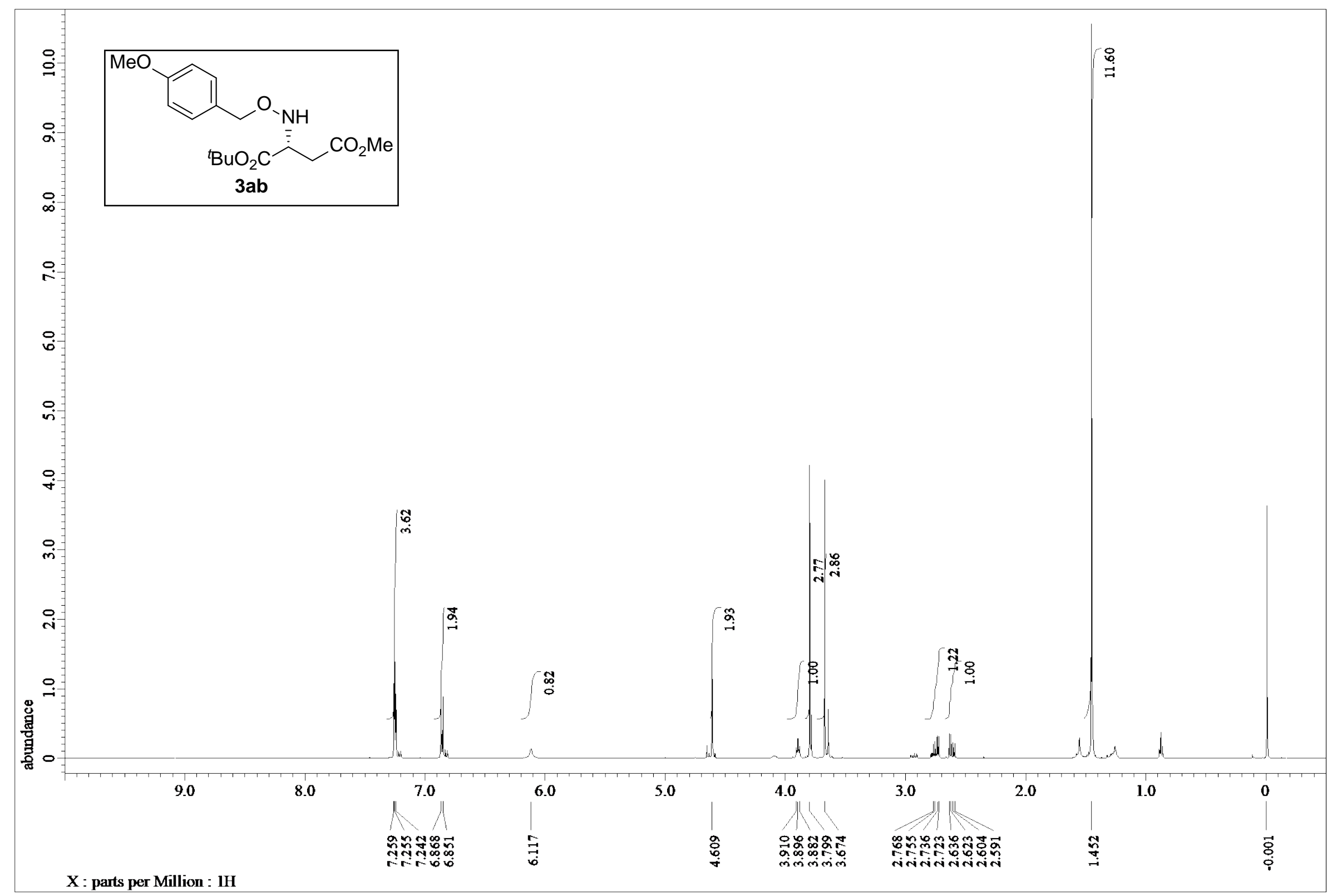




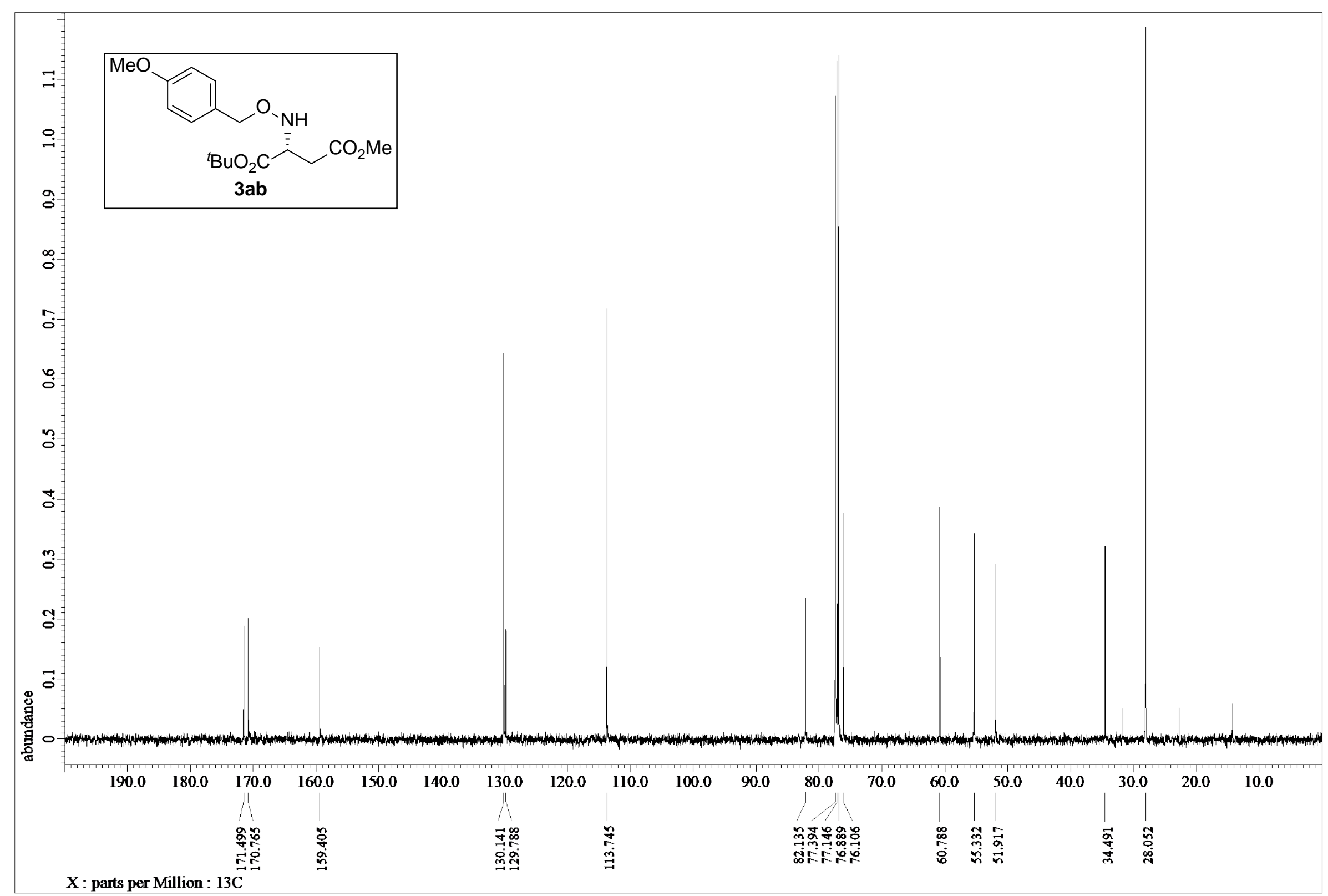




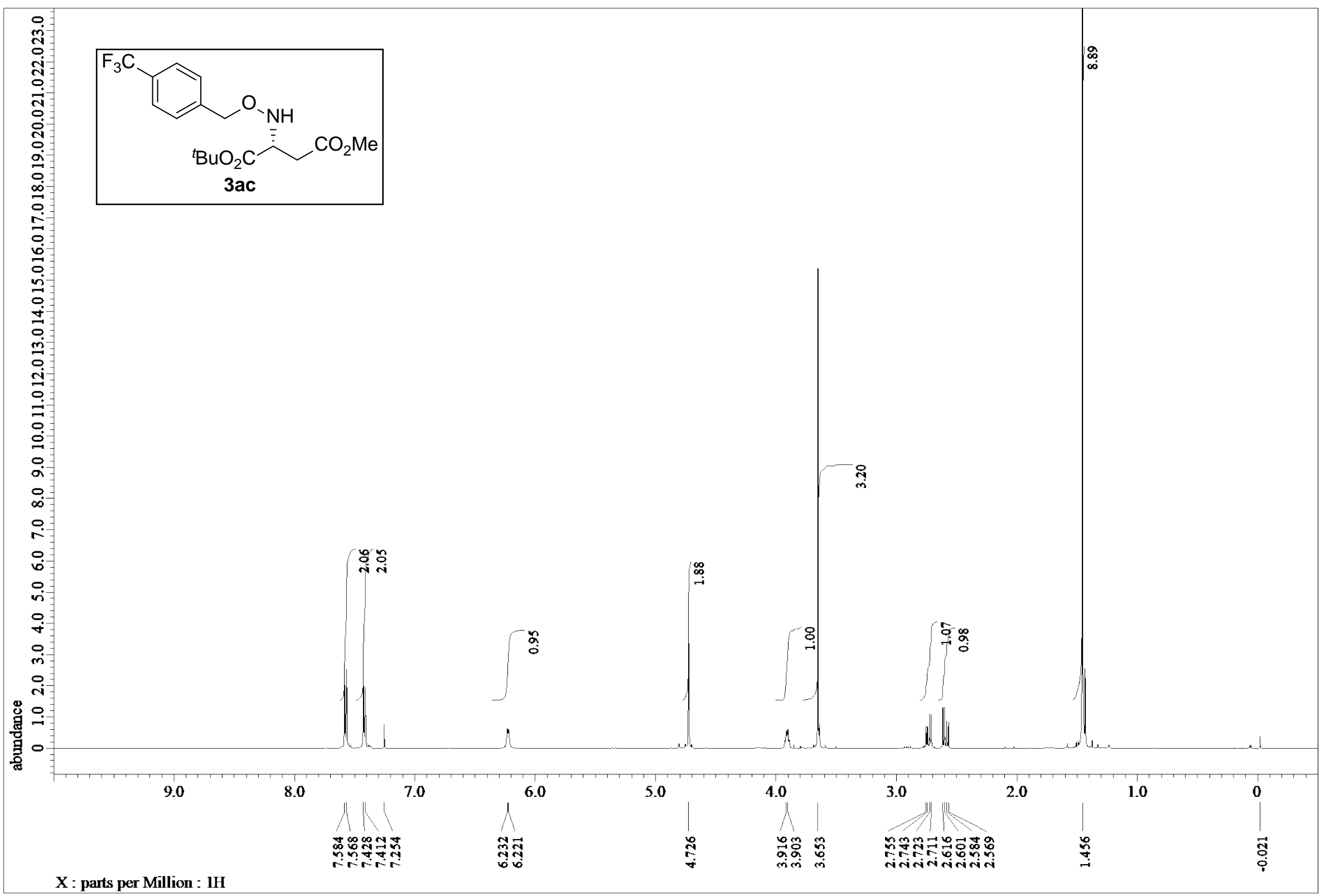




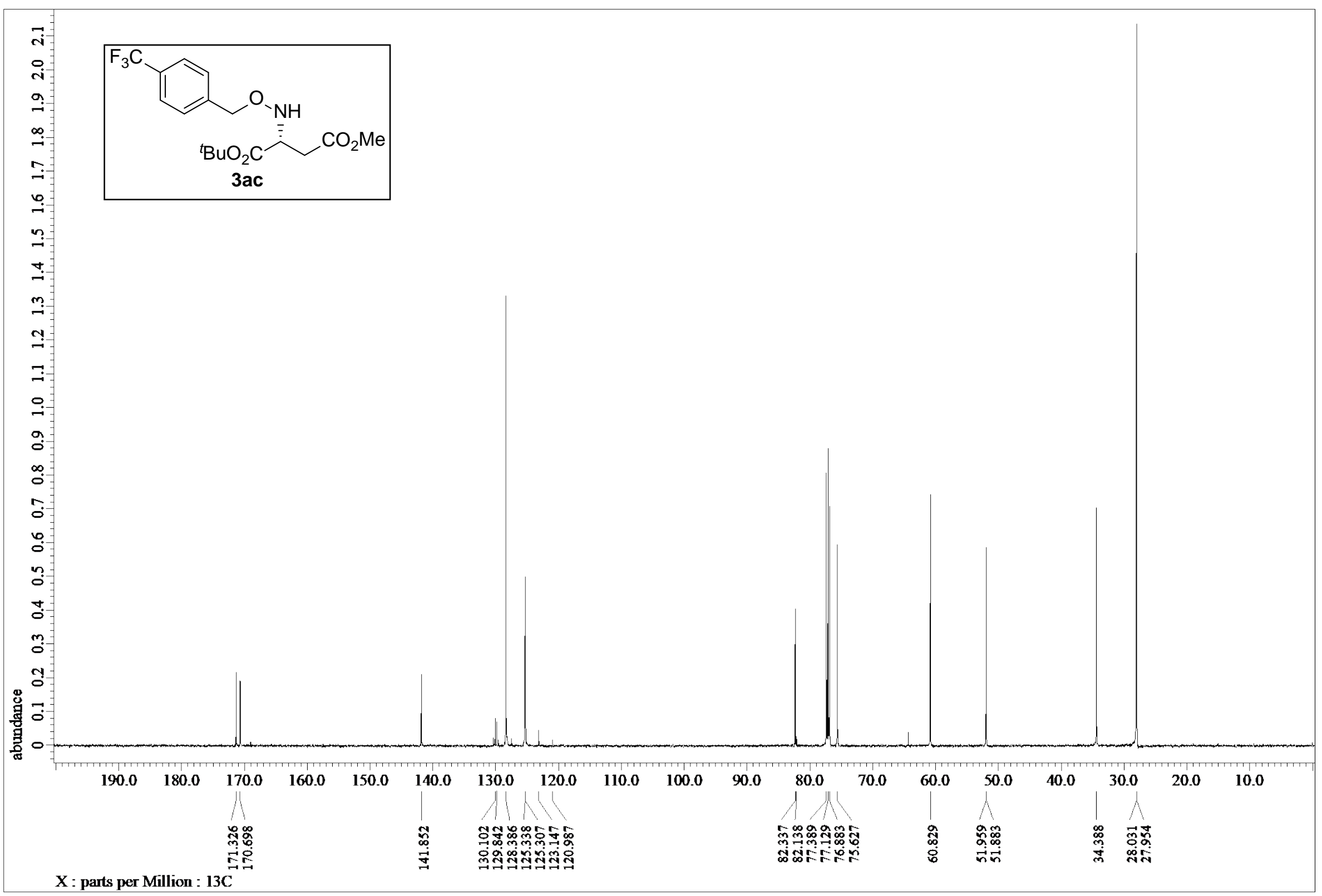




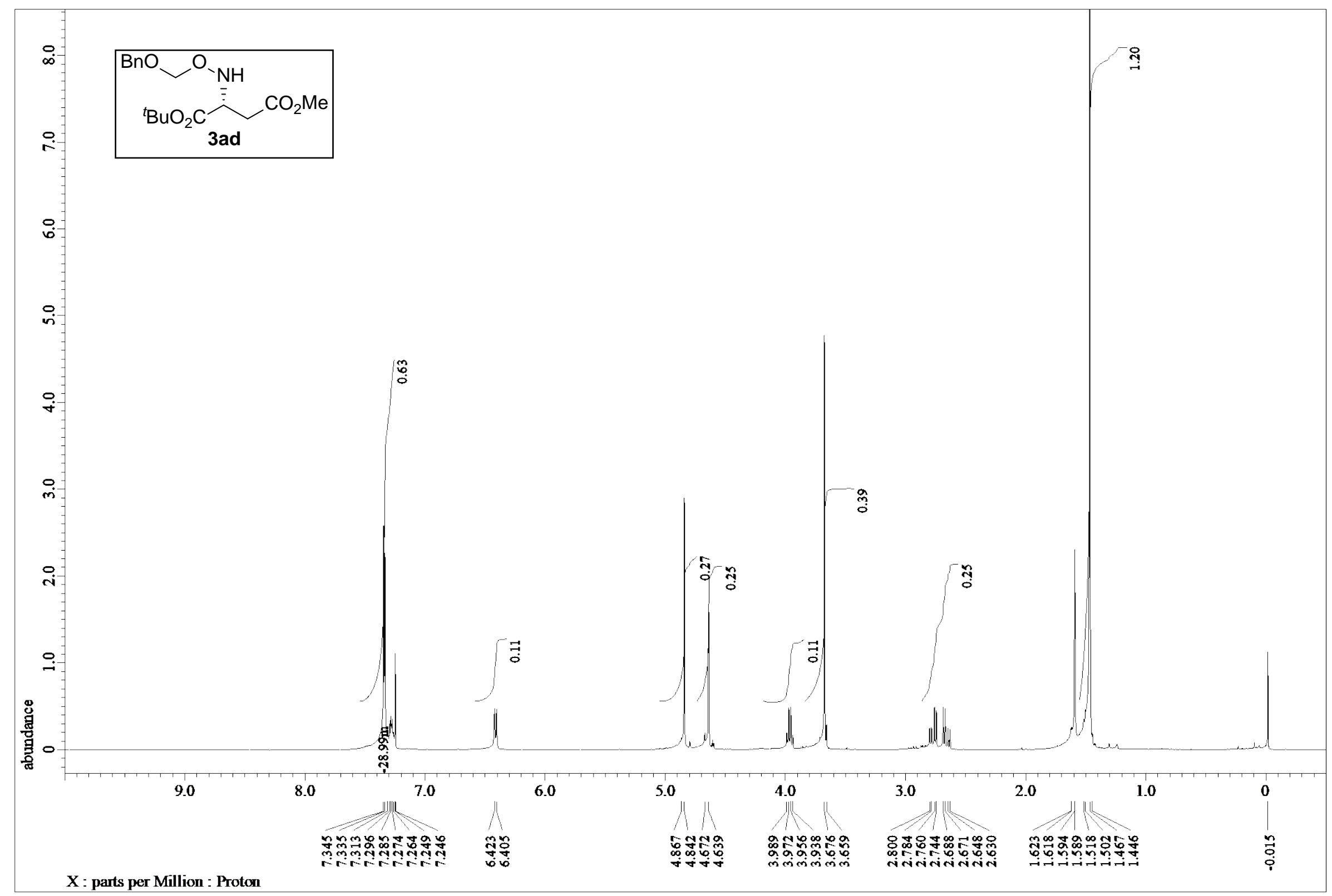




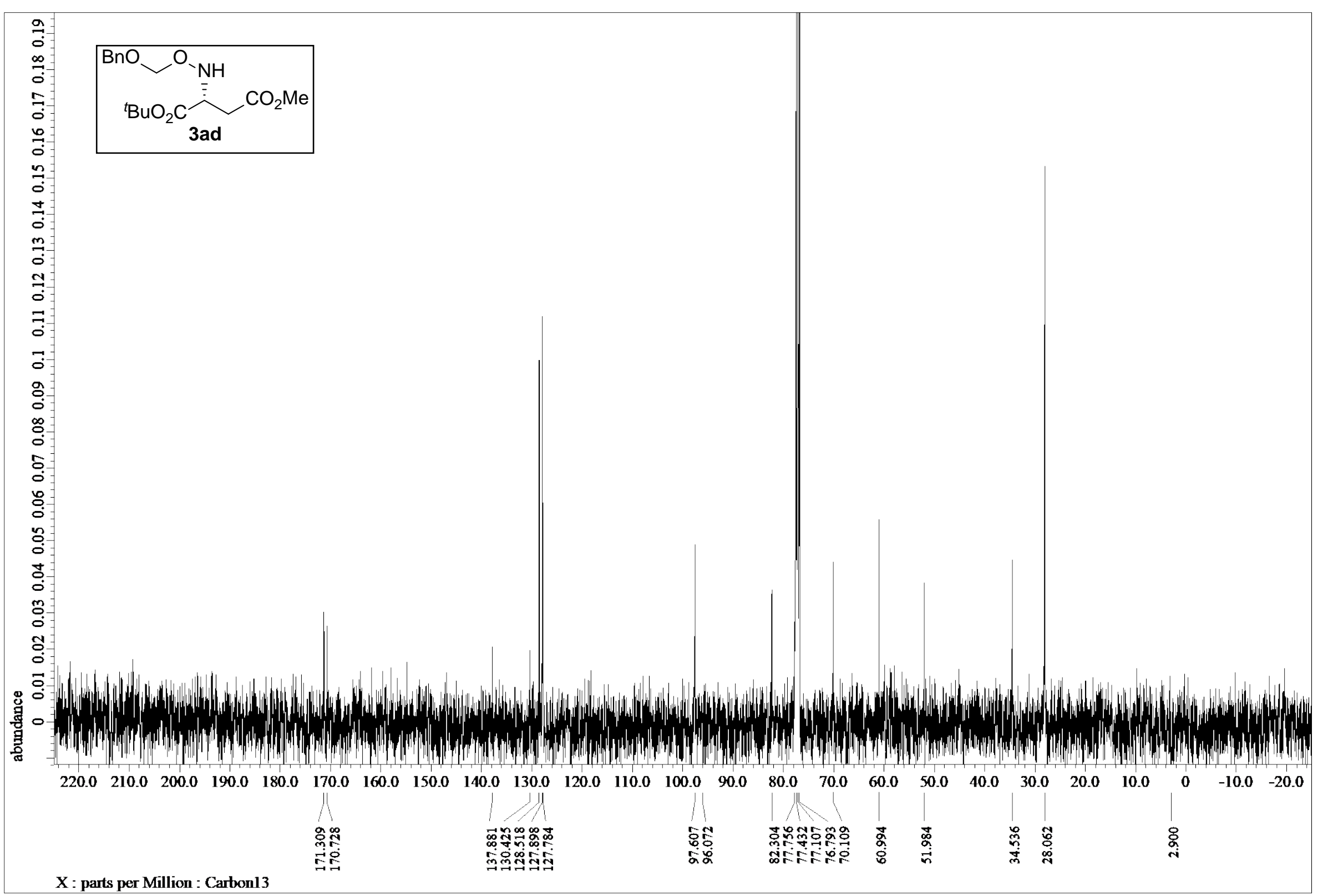




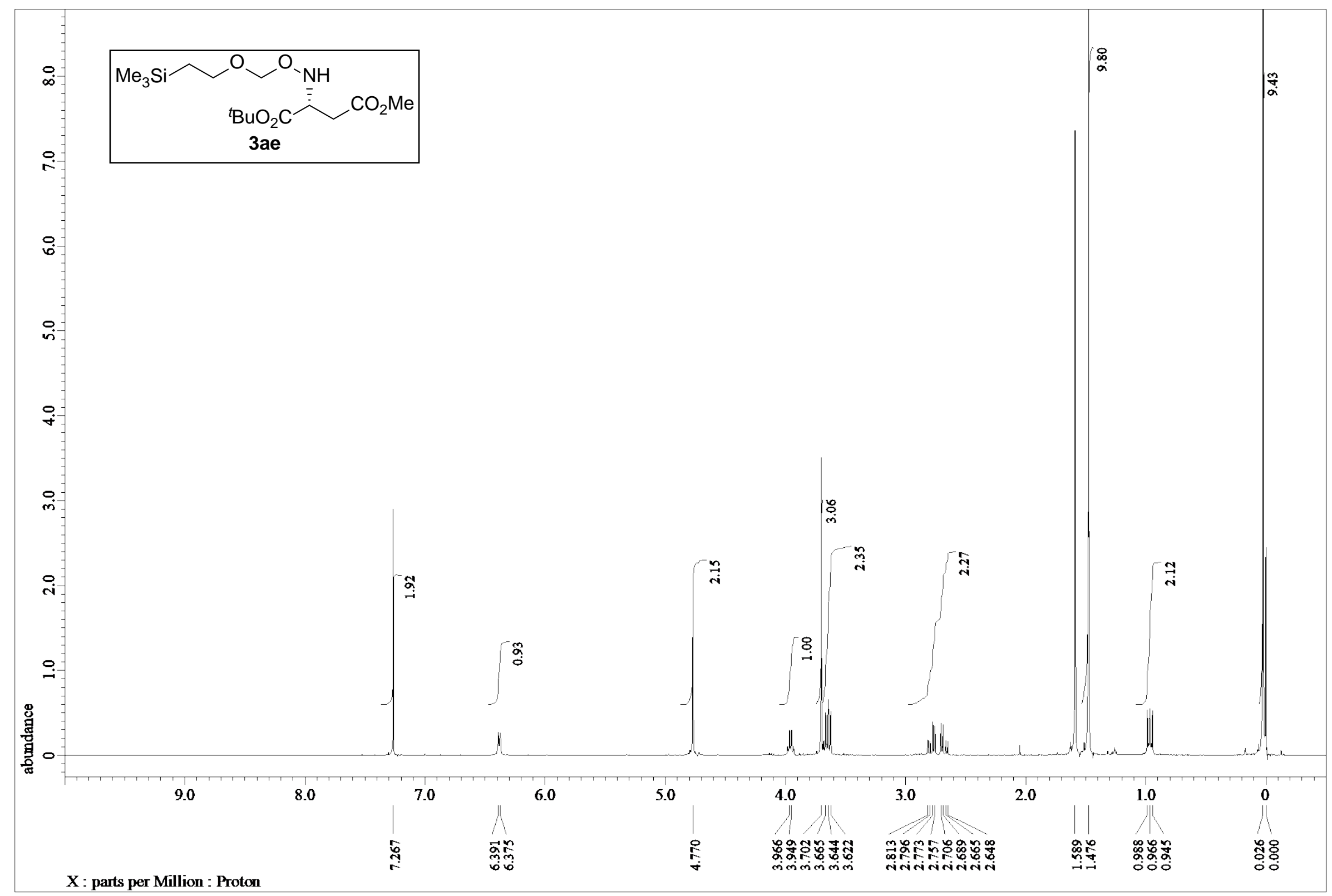




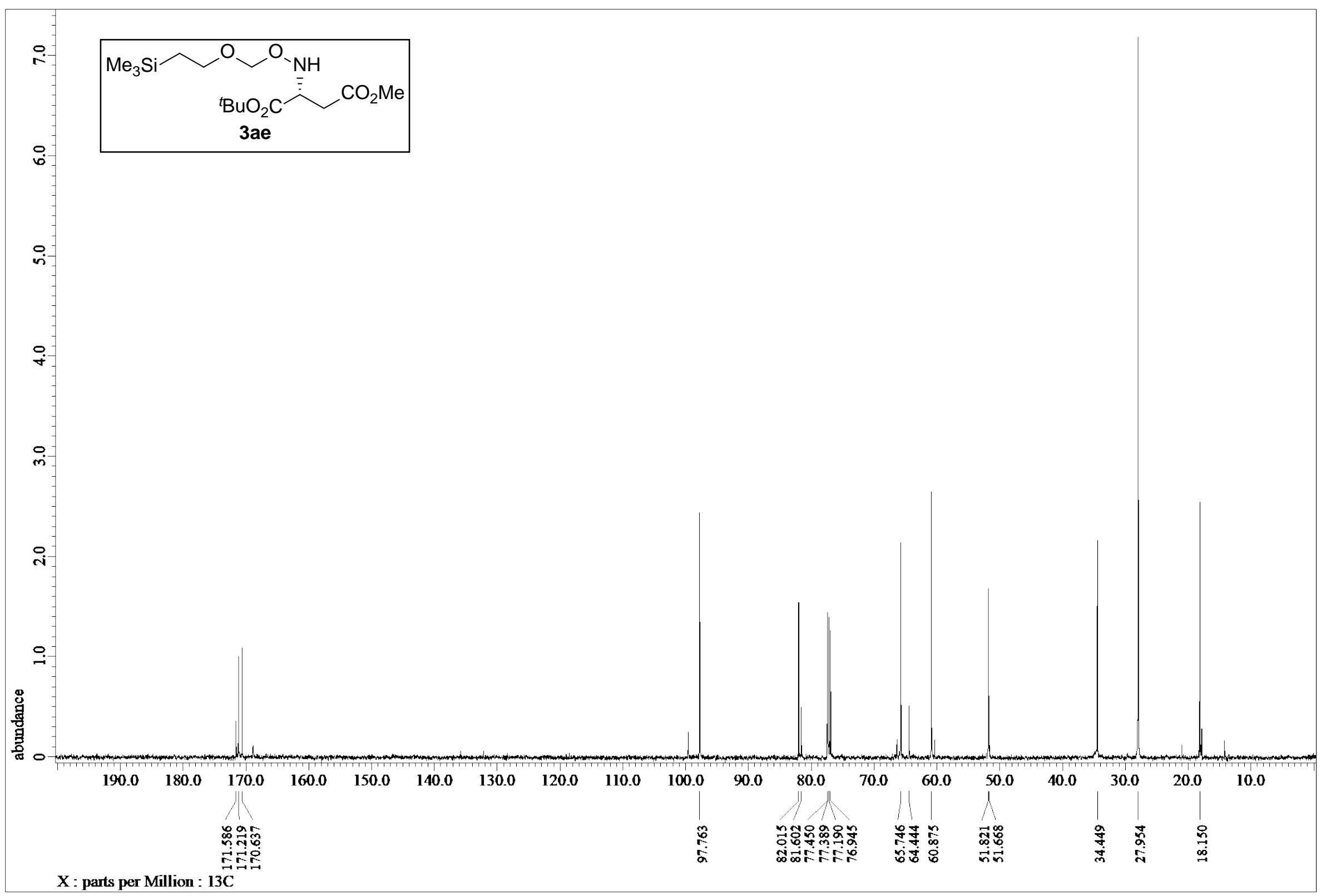




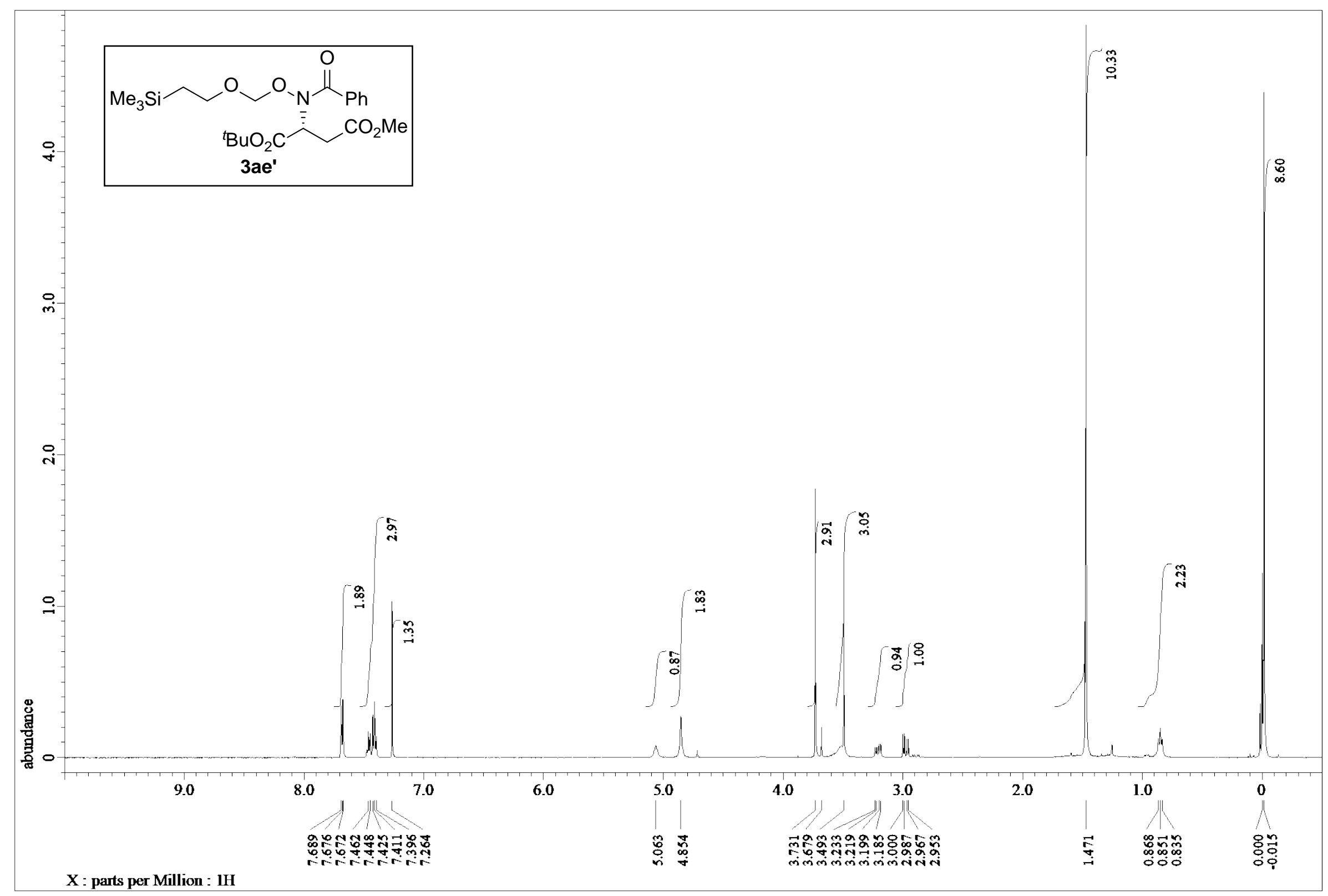




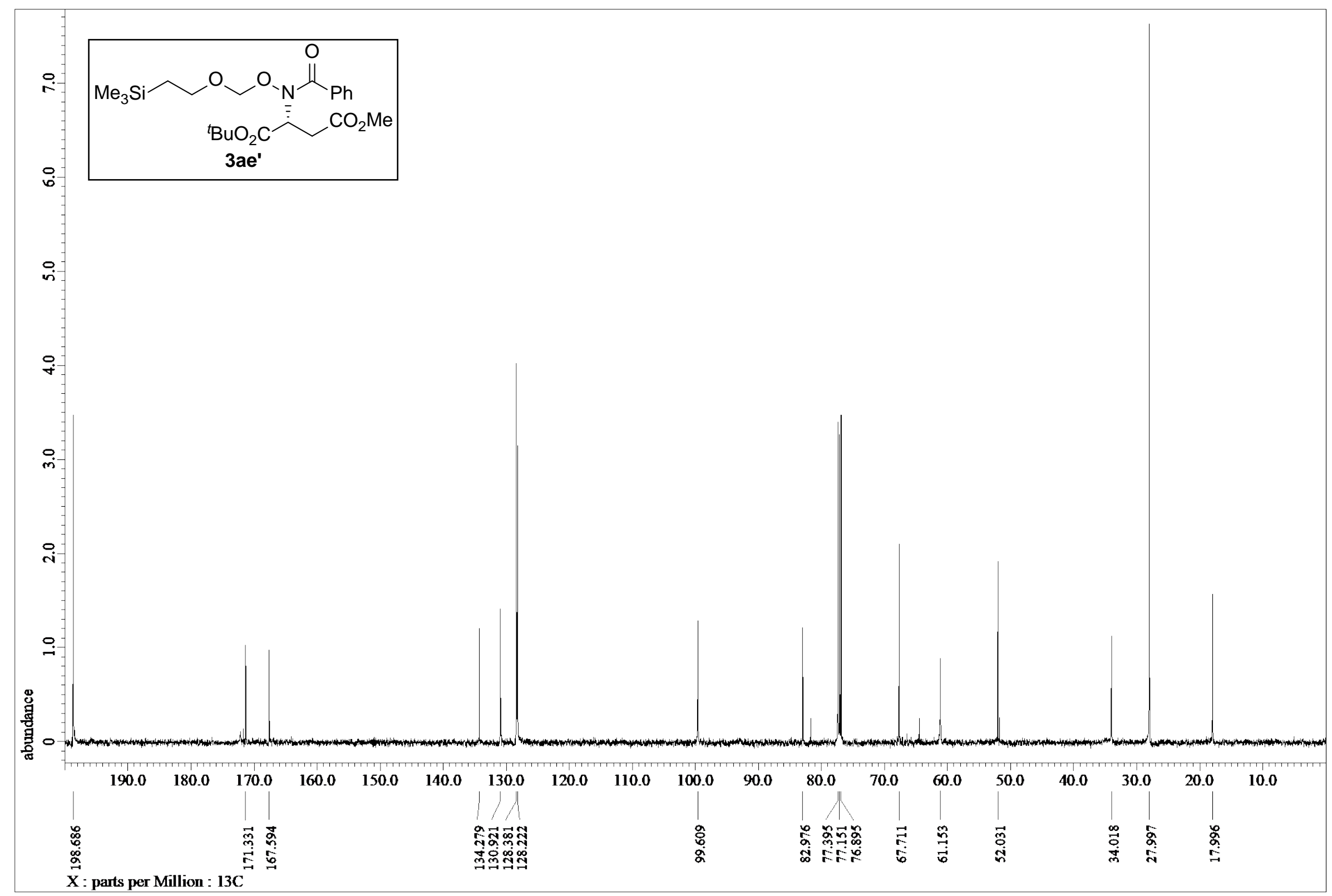




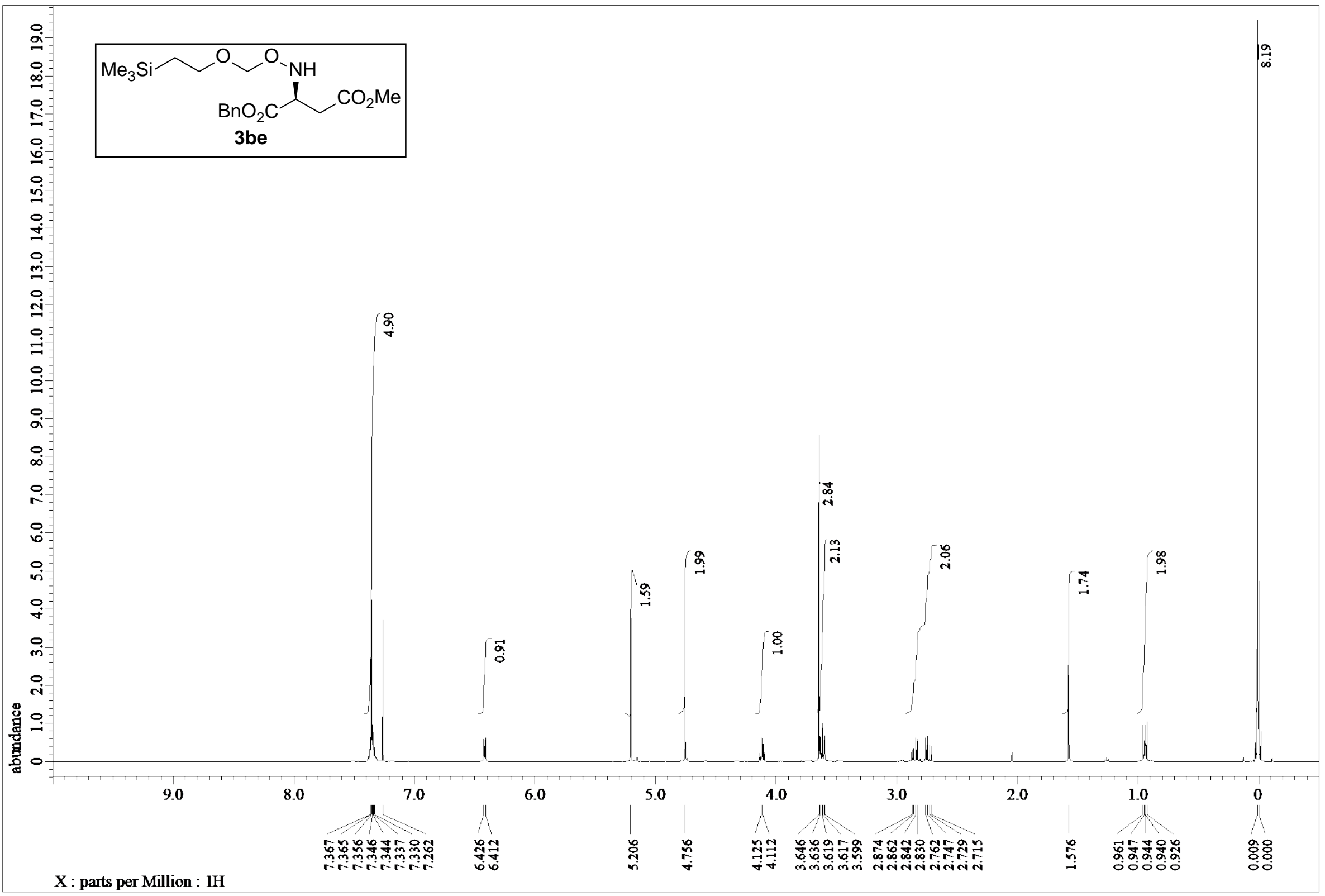




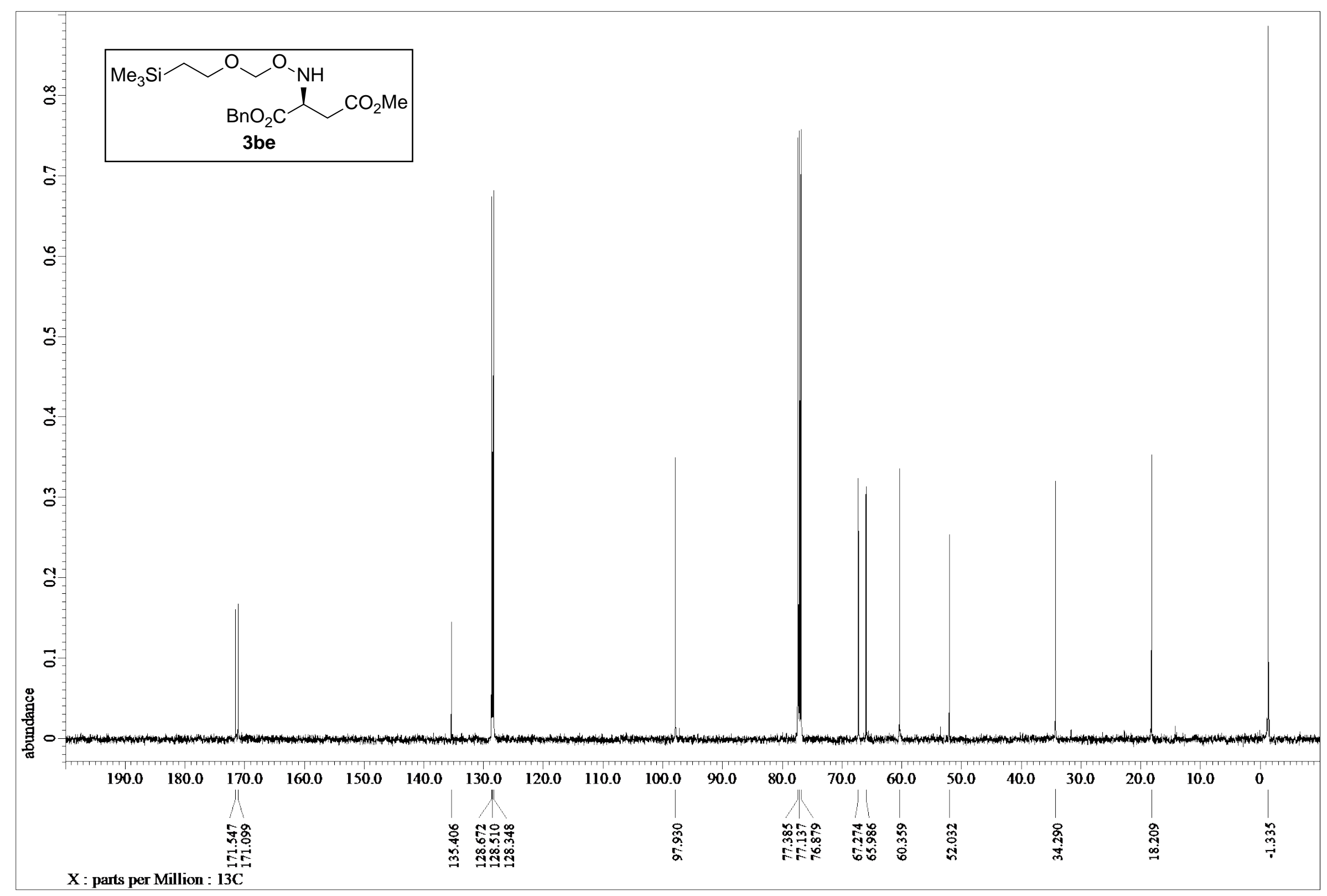




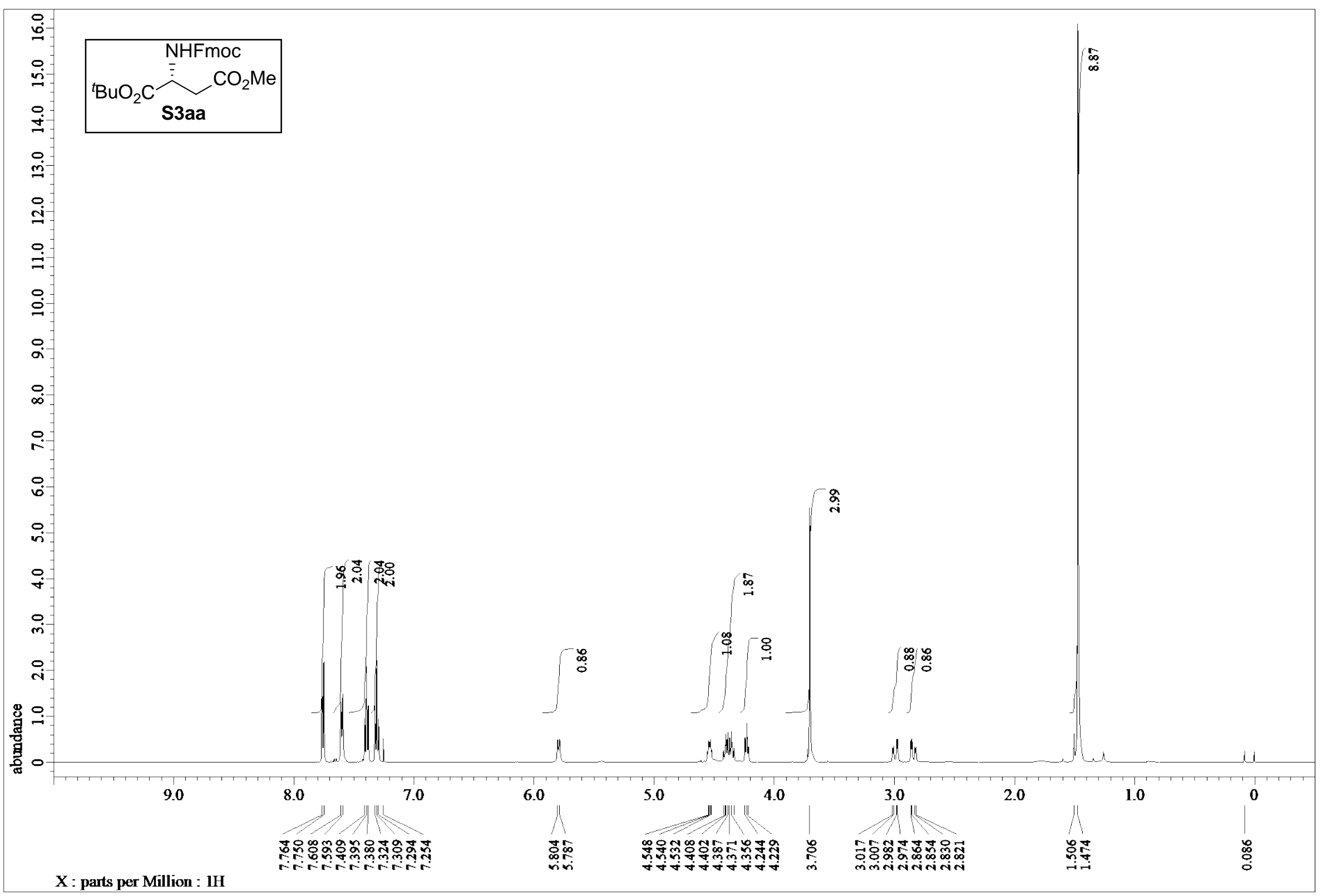




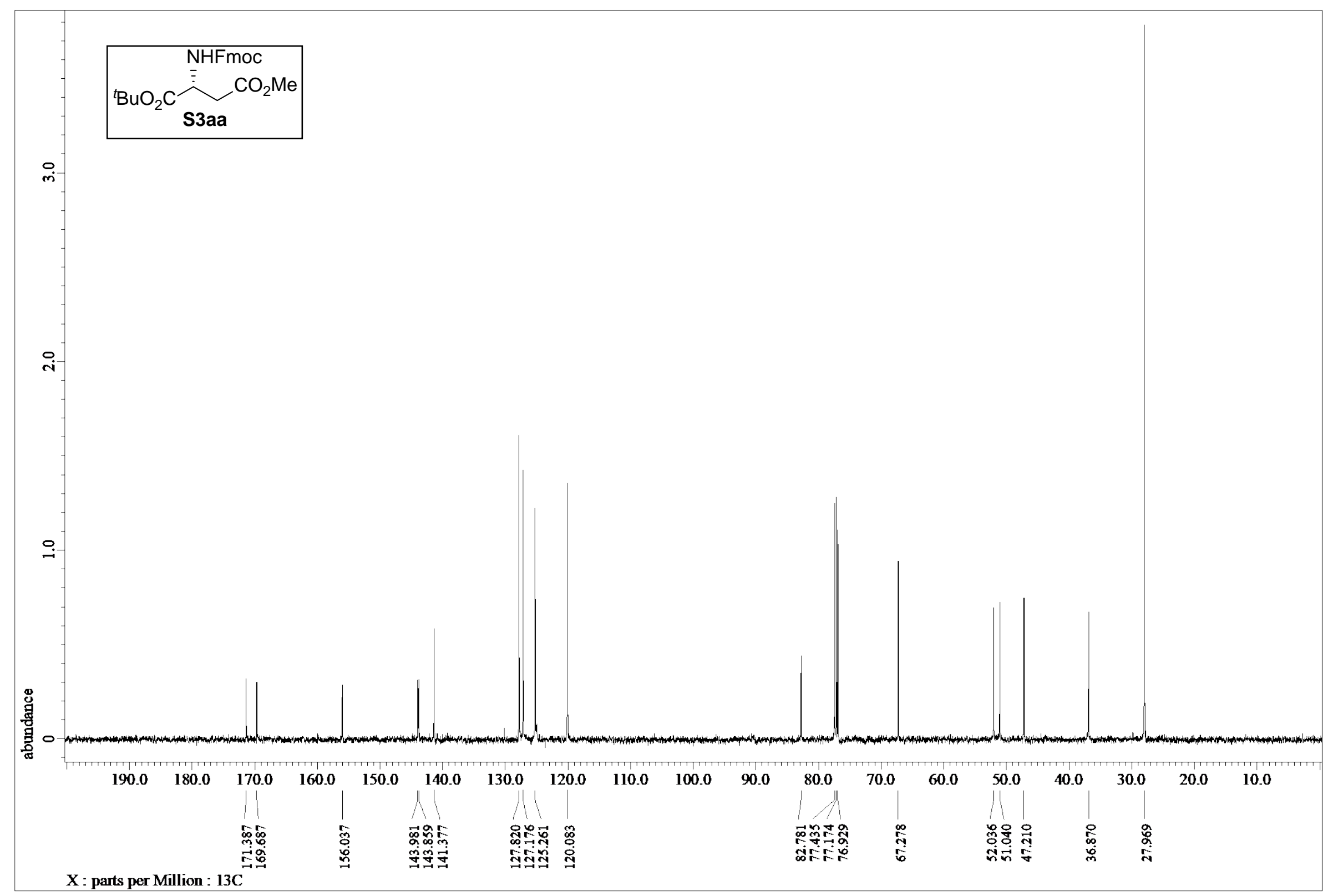




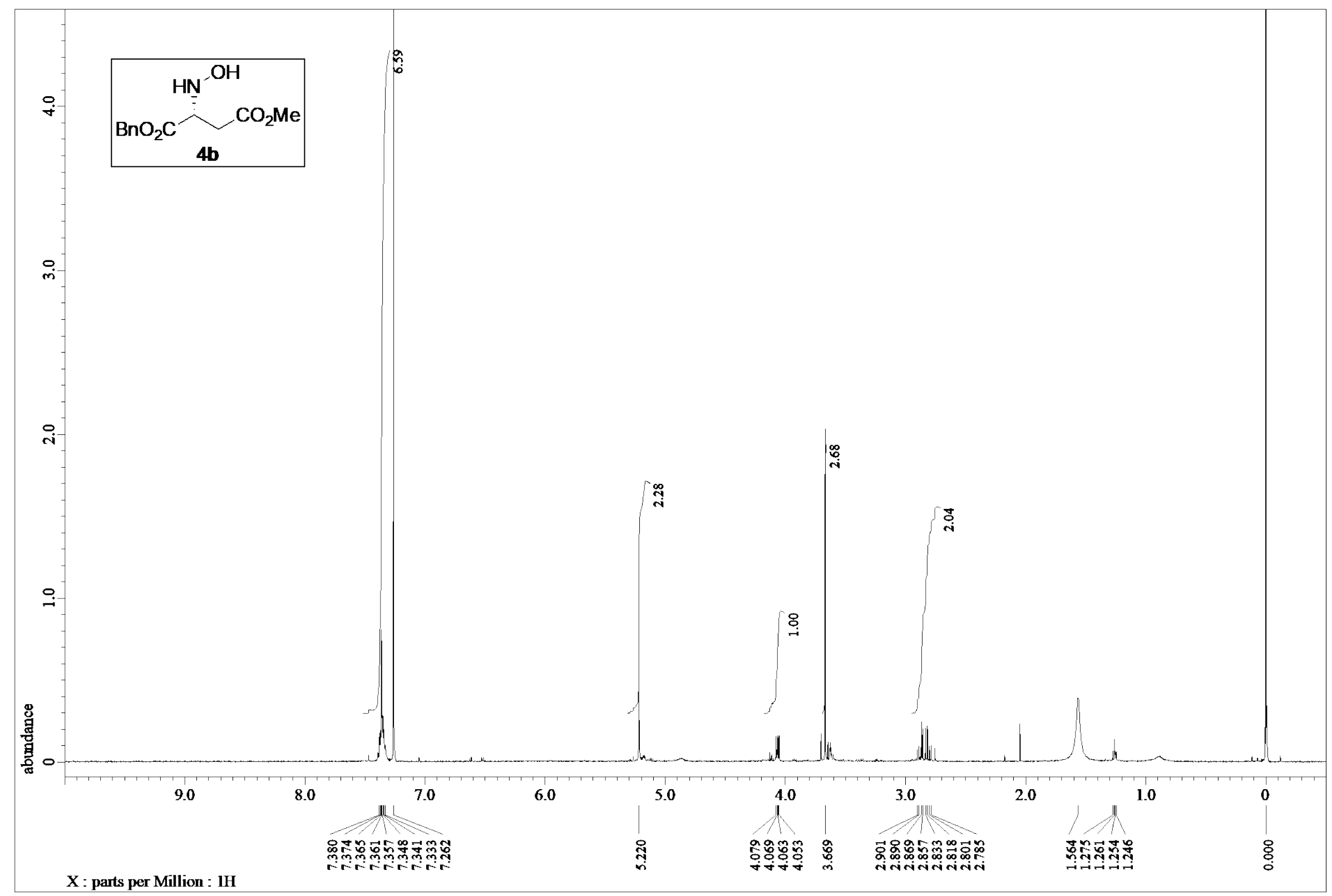




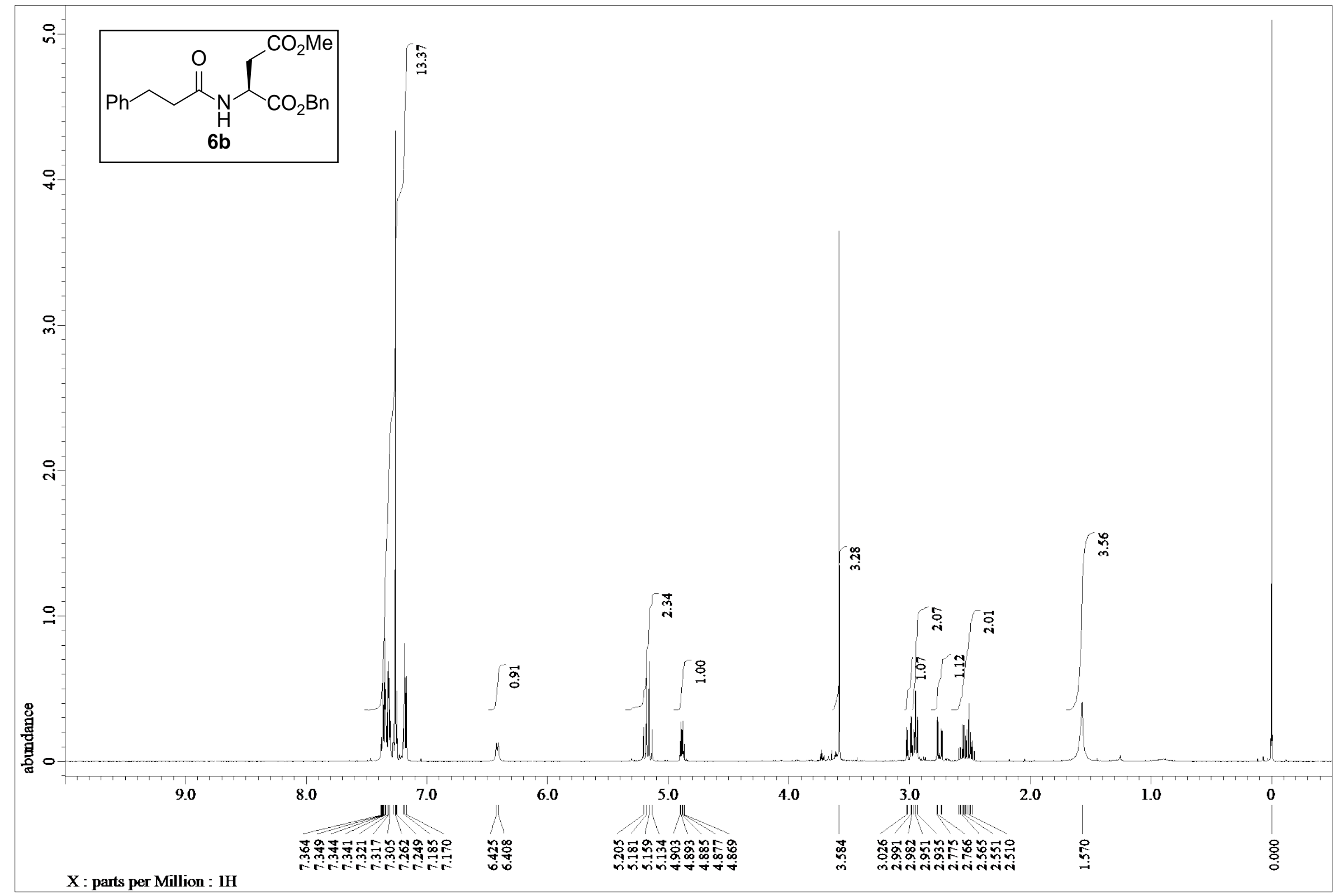




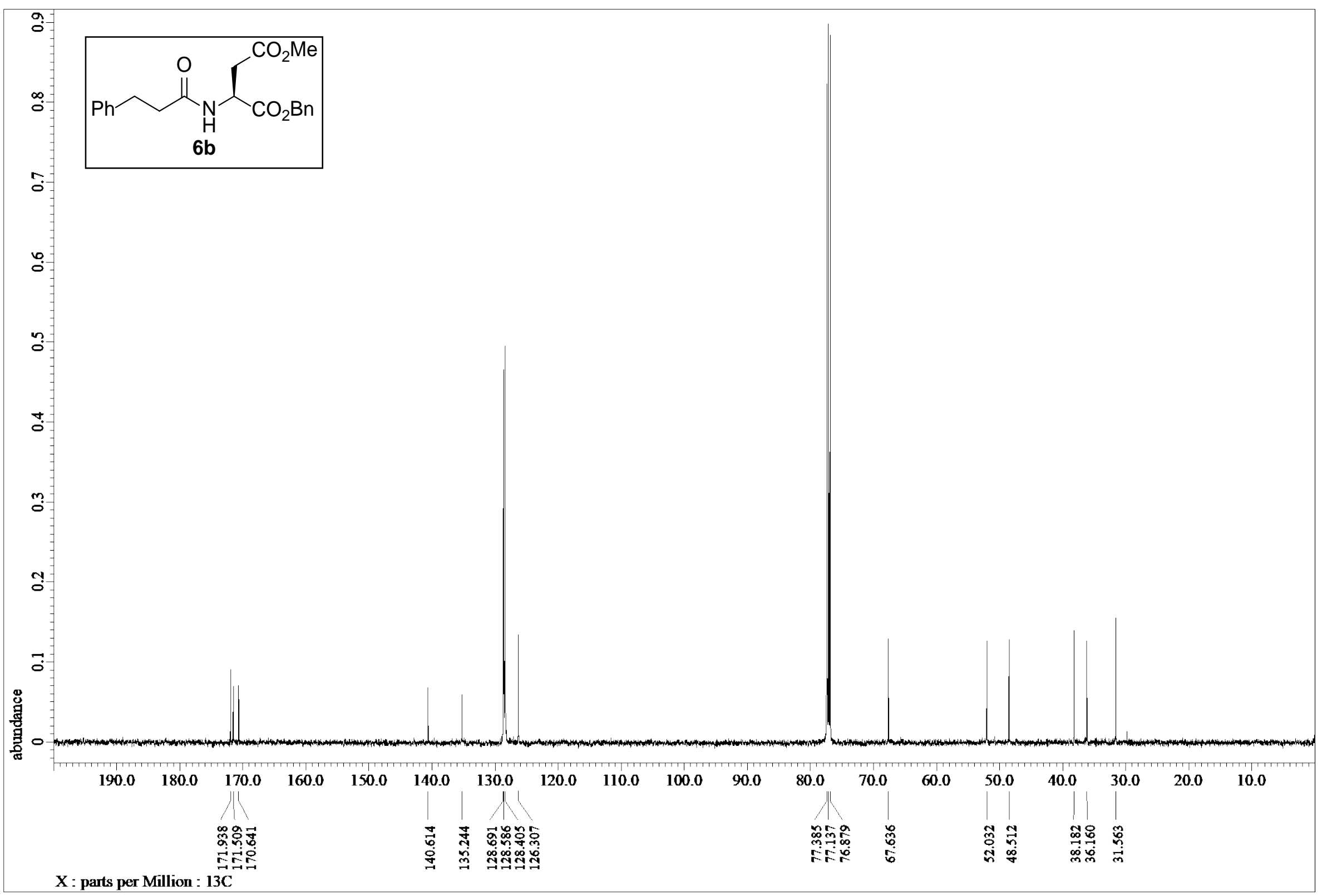




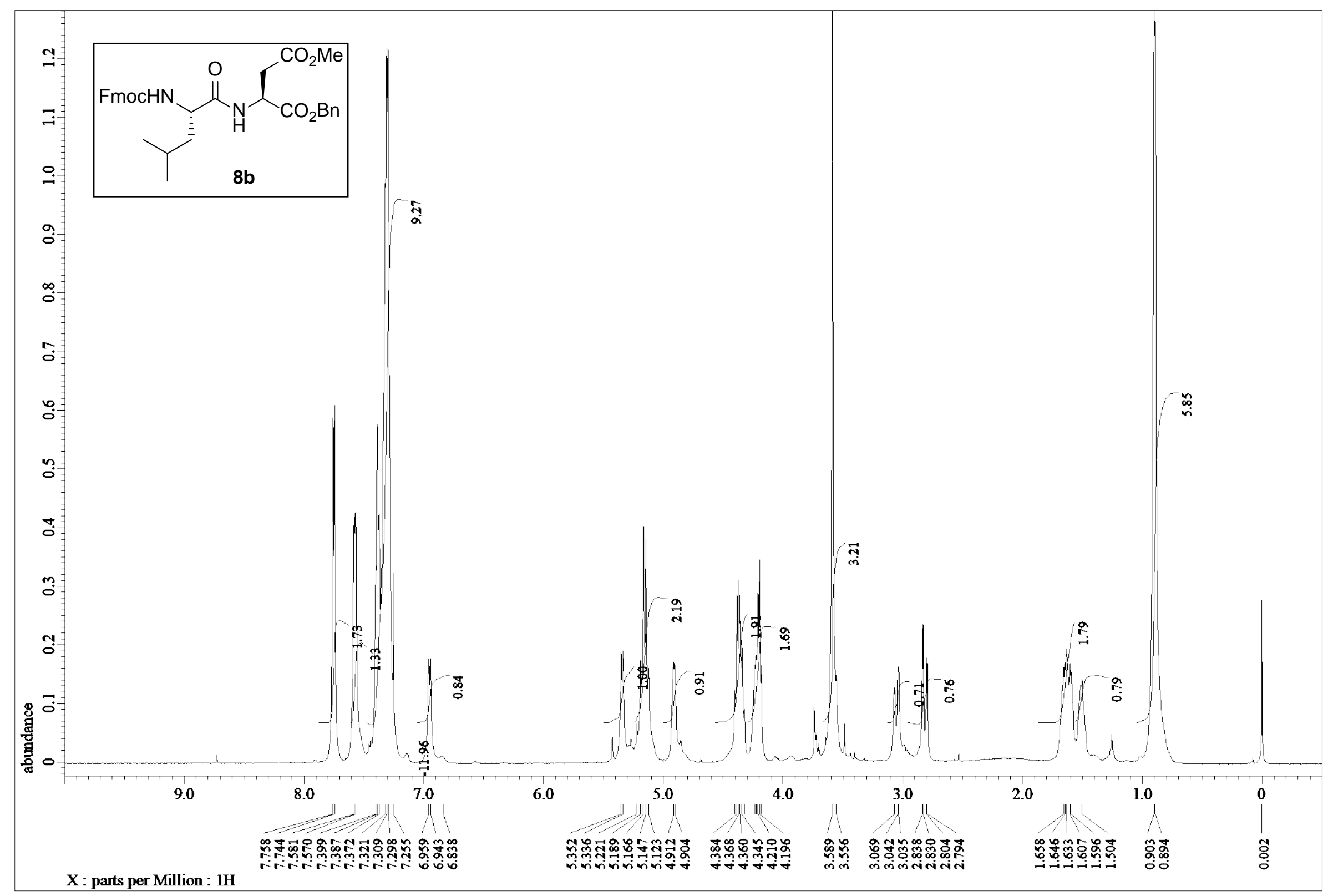




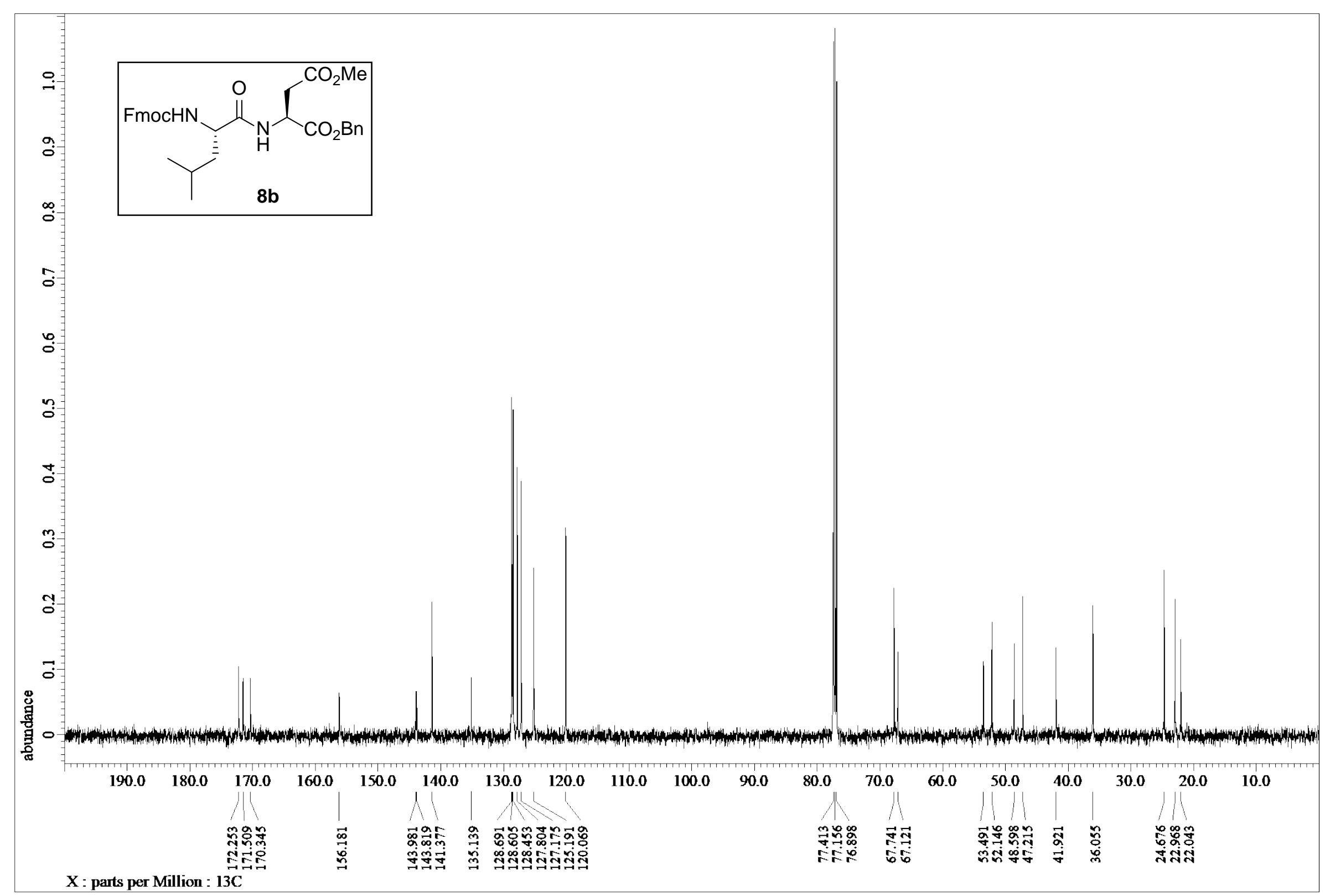




\section{(G) HPLC Traces}

${ }^{t} \mathrm{BuO}_{2} \overbrace{\text { 3aa }}^{\mathrm{BnO}_{-}^{\mathrm{NH}}} \mathrm{CO}_{2} \mathrm{Me}$

$\langle$ Chromatogram〉

chromatogram

25 C:¥data_131217¥murakami¥HM0025-IB004.lcd

mAU

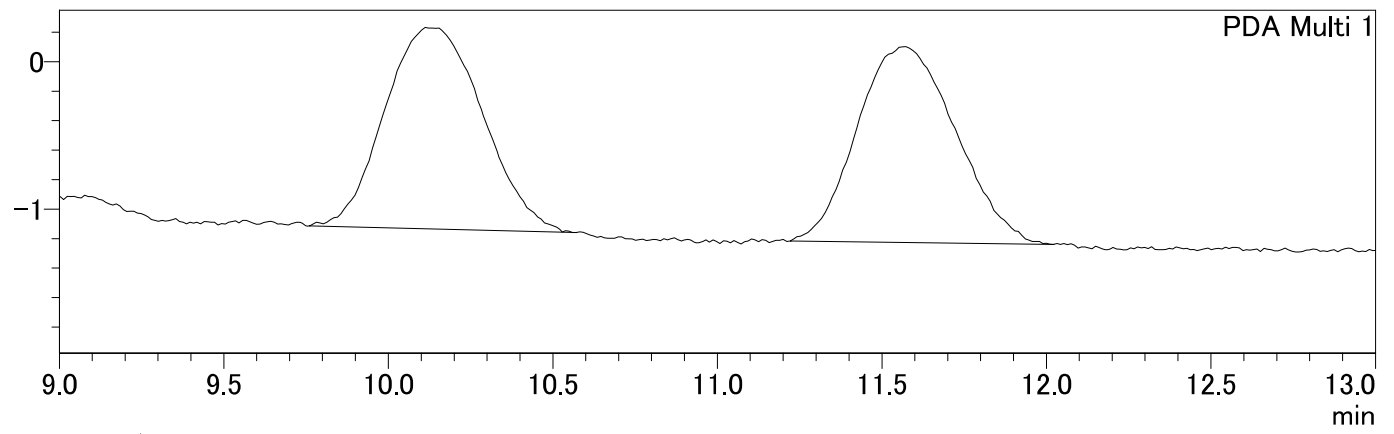

1 PDA Multi $1 / 254 \mathrm{~nm} 4 \mathrm{~nm}$

$\langle$ Peak Report>

PDA Ch1 254nm 4nm

peak table C:¥data_131217¥murakami¥HM0025-IB004.lcd

\begin{tabular}{|r|r|r|r|}
\hline peak\# & retention time $(\min )$ & area & area (\%) \\
\hline 1 & 10.110 & 28220 & 50.566 \\
2 & 11.563 & 27589 & 49.434 \\
\hline
\end{tabular}

$\langle$ Chromatogram $\rangle$

chromatogram

HM0033-C C:¥data_131217¥murakami¥HM0033-C-IA.Icd

$\mathrm{mAU}$

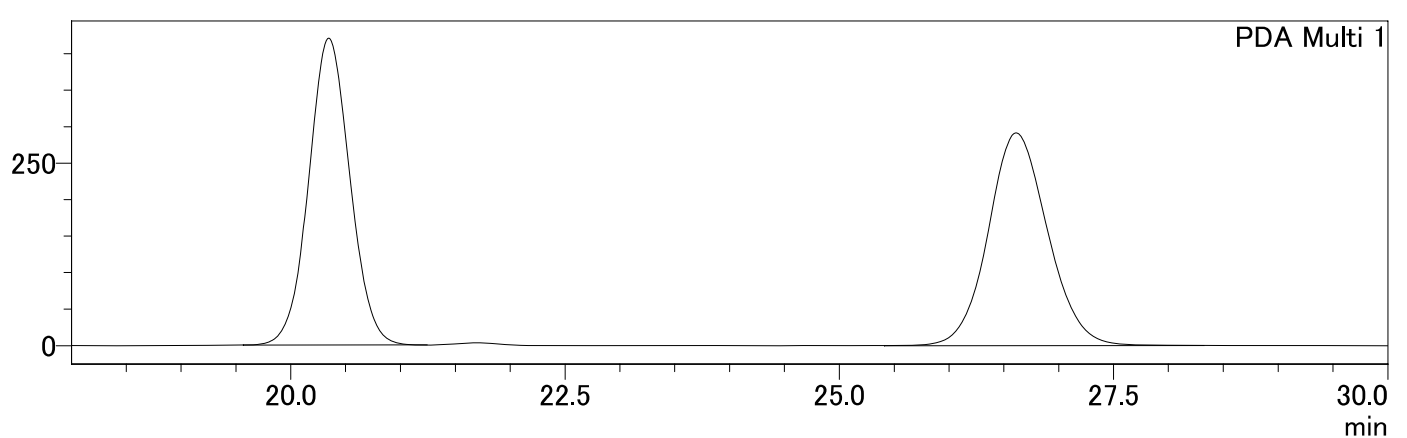

1 PDA Multi 1 / 254nm 4nm

$\langle$ Peak Report>

peak table C:¥data_131217¥murakami¥HM0033-C-IA.Icd

PDA Ch1 254nm 4nm

peak\#

\begin{tabular}{|r|r|r|r} 
& retention time $(\min )$ & area & area $(\%)$ \\
\hline 1 & 20.340 & 11004240 & 50.134 \\
2 & 26.606 & 10945554 & 49.866 \\
\hline
\end{tabular}




$\mathrm{BnO}_{2} \overbrace{\text { 3ba }}^{\mathrm{BnO}_{-}^{\mathrm{NH}}} \mathrm{CO}_{2} \mathrm{Me}$

$\langle$ Chromatogram〉

chromatogram

HM0121-C C:¥data_131217¥murakami¥HM0121-2-C-IB001.lcd

mAU

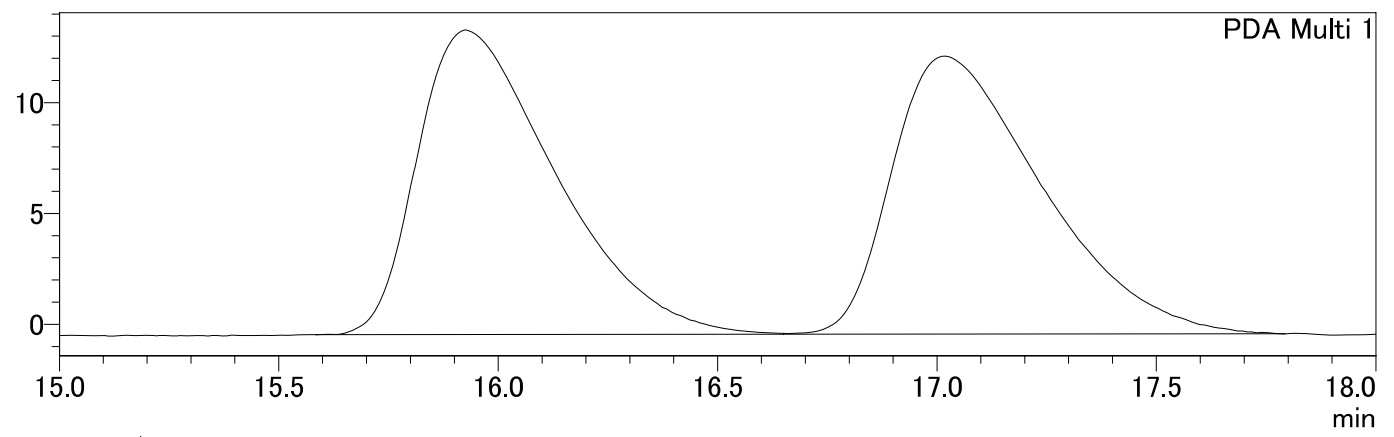

1 PDA Multi $1 / 254 \mathrm{~nm} 4 \mathrm{~nm}$

〈Peak Report>

PDA Ch1 254nm 4nm

peak table C:¥data_131217¥murakami¥HM0121-2-C-IB001.lcd

\begin{tabular}{|r|r|r|r|}
\hline peak\# & retention time $(\min )$ & area & area (\%) \\
\hline 1 & 15.920 & 300238 & 50.167 \\
2 & 17.012 & 298243 & 49.833 \\
\hline
\end{tabular}

$\langle$ Chromatogram〉

chromatogram

HM0125-C C:¥data_131217¥murakami¥HM0125-C-IB001.lcd

$\mathrm{mAU}$

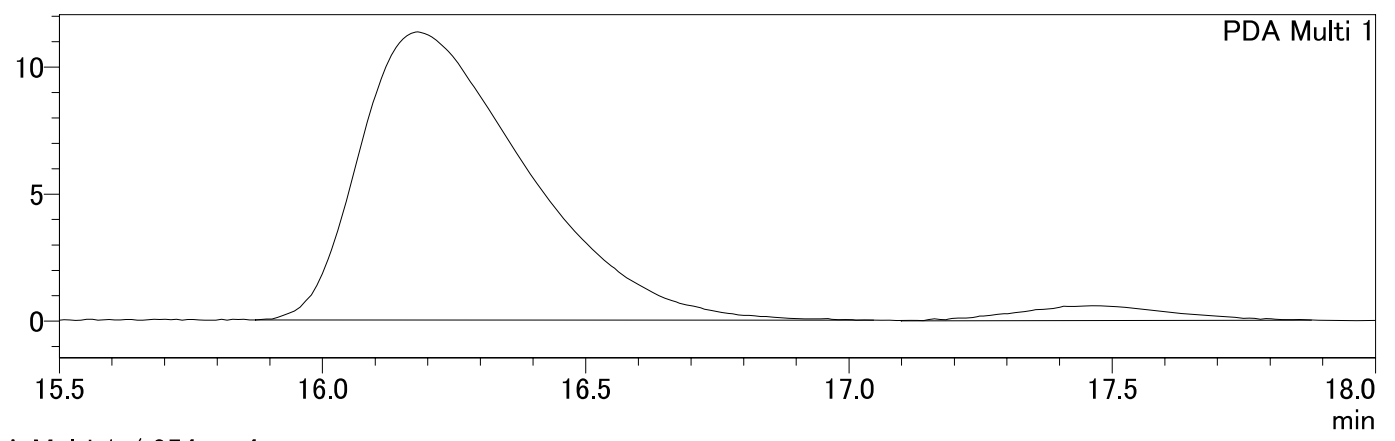

1 PDA Multi $1 / 254 \mathrm{~nm} 4 \mathrm{~nm}$

$\langle$ Peak Report>

PDA Ch1 254nm 4nm

peak table C:¥data_131217¥murakami¥HM0125-C-IB001.lcd

\begin{tabular}{|r|r|r|r|}
\hline peak\# & retention time $(\min )$ & area & area (\%) \\
\hline 1 & 16.175 & 249368 & 95.468 \\
2 & 17.454 & 11837 & 4.532 \\
\hline
\end{tabular}




$\mathrm{EtO}_{2} \mathrm{C} \overbrace{3 \mathbf{c a}}^{\mathrm{BnO}_{-\mathrm{NH}}} \mathrm{CO}_{2} \mathrm{Me}$

$\langle$ Chromatogram〉

chromatogram

HM0051-C C:¥data_131217¥murakami¥HM0051-C-IB001.lcd

$\mathrm{mAU}$

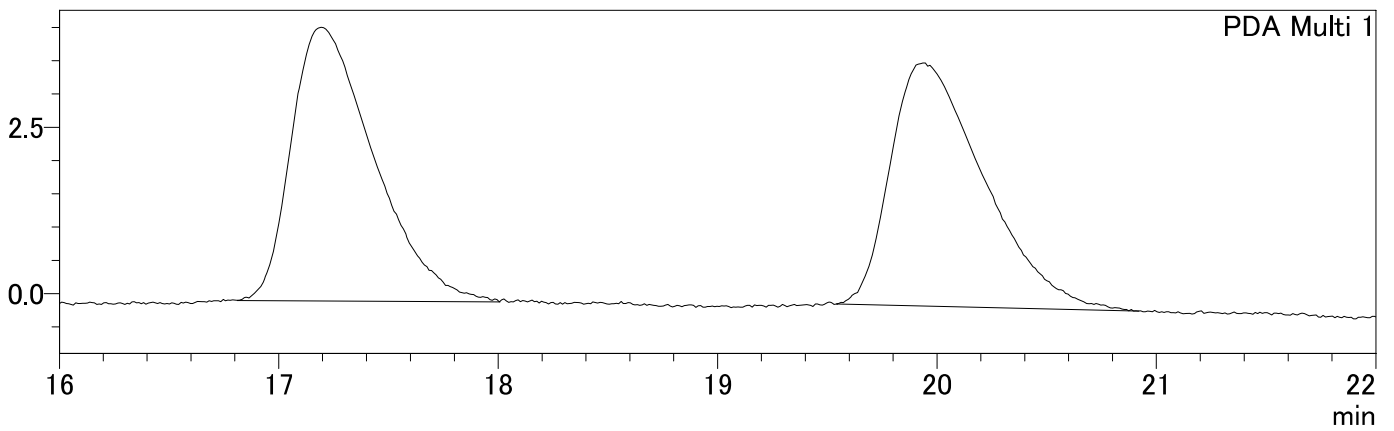

1 PDA Multi $1 / 254 \mathrm{~nm} 4 \mathrm{~nm}$

$\langle$ Peak Report>

PDA Ch1 254nm 4nm

peak table C:¥data_131217¥murakami¥HM0051-C-IB001.lcd

\begin{tabular}{|r|r|r|r|}
\hline peak\# & retention time $(\min )$ & area & area (\%) \\
\hline 1 & 17.190 & 107411 & 49.860 \\
2 & 19.936 & 108013 & 50.140 \\
\hline
\end{tabular}

\section{$\langle$ Chromatogram〉}

chromatogram

HM0124-C C:¥data_131217¥murakami¥HM0124-C-IB001.lcd

mAU

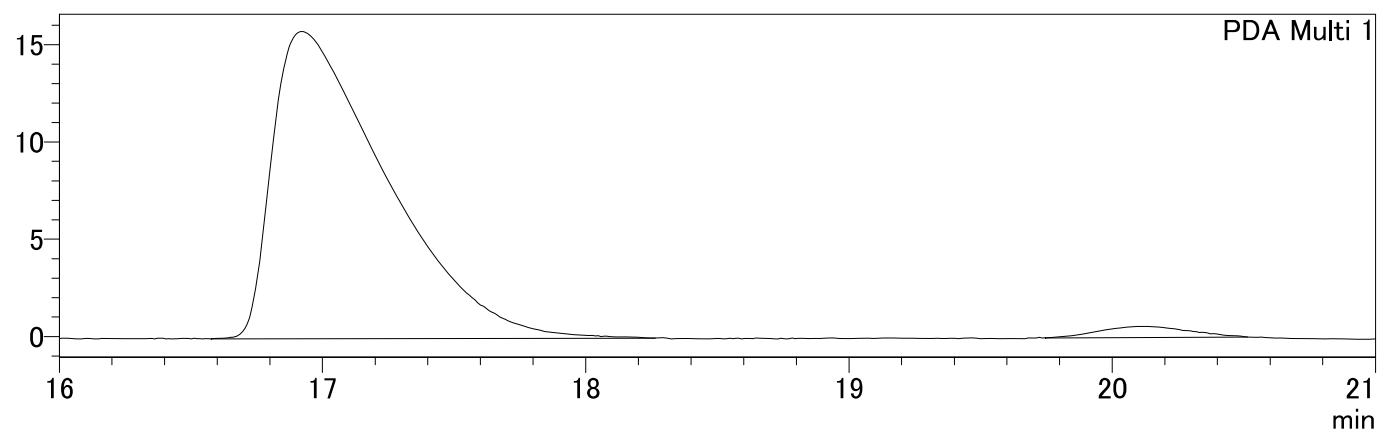

1 PDA Multi 1 / 254nm 4nm

〈Peak Report>

peak table C:¥data_131217¥murakami¥HM0124-C-IB001.lcd

PDA Ch1 254nm 4nm

peak\# retention time $(\min )$

16.916
20.131

area area (\%) 20.131

476565

97.175 13852 2.825 


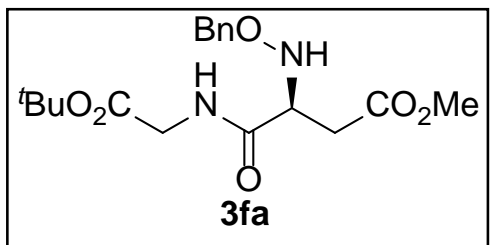

$\langle$ Chromatogram〉

chromatogram

HM0525 C:¥data_131217¥murakami¥HM0525-IB003.lcd

$\mathrm{mAU}$

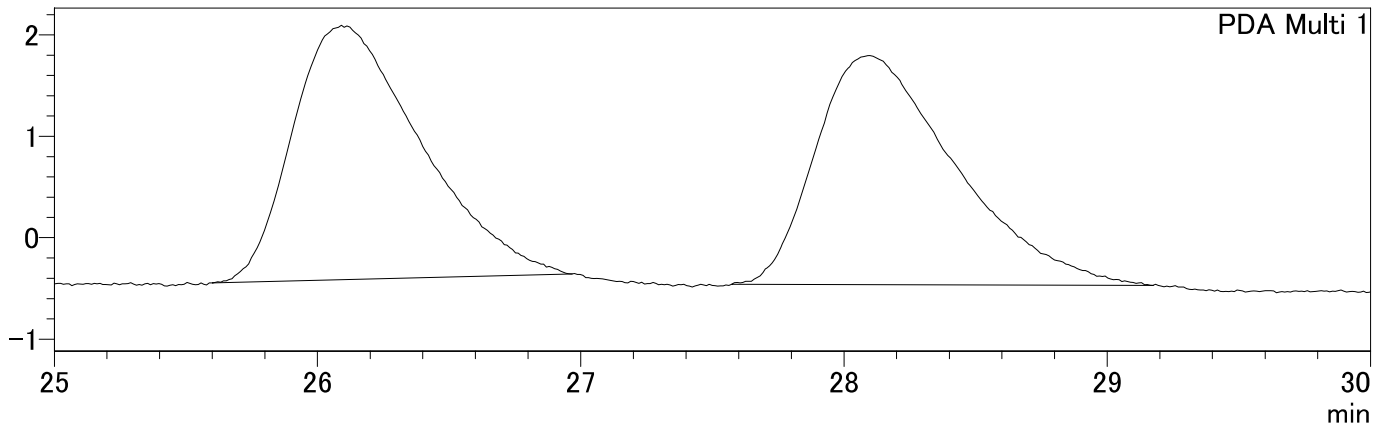

1 PDA Multi $1 / 254 \mathrm{~nm} 4 \mathrm{~nm}$

$\langle$ Peak Report>

peak table C:¥data 131217¥murakami¥HM0525-IB003.Icd

PDA Ch1 254nm 4nm

peak\#

\begin{tabular}{r|r|r} 
retention time $(\min )$ & area & area $(\%)$ \\
\hline 26.085 & 84655 & 50.344 \\
28.090 & 83498 & 49.656
\end{tabular}

\section{$\langle$ Chromatogram〉}

chromatogram

HM0526 C:¥data_131217¥murakami¥HM0526-IB001.lcd

mAU

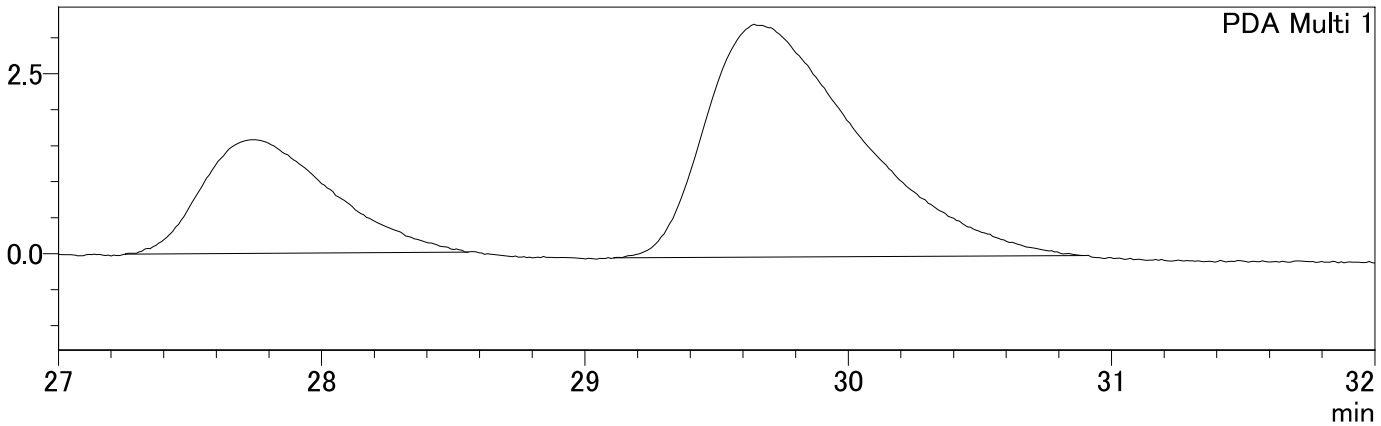

1 PDA Multi $1 / 254 \mathrm{~nm} 4 \mathrm{~nm}$

$\langle$ Peak Report>

PDA Ch1 254nm 4nm

peak table C:¥data_131217¥murakami¥HM0526-IB001.lcd

peak\#

1 .

retention time $(\mathrm{min})$

\begin{tabular}{|r|r|r}
\hline 27.732 & \multicolumn{1}{|c|}{ area } & area $(\%)$ \\
\hline 29.637 & 130924 & 29.314 \\
& 13093 & 70.686
\end{tabular}




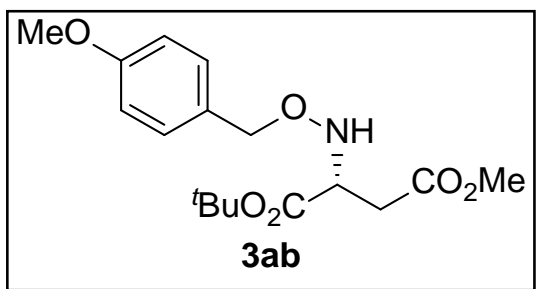

\section{$\langle$ Chromatogram〉}

chromatogram

HM0551 C:¥data_131217¥murakami¥HM0551-IB.Icd

$\mathrm{mAU}$

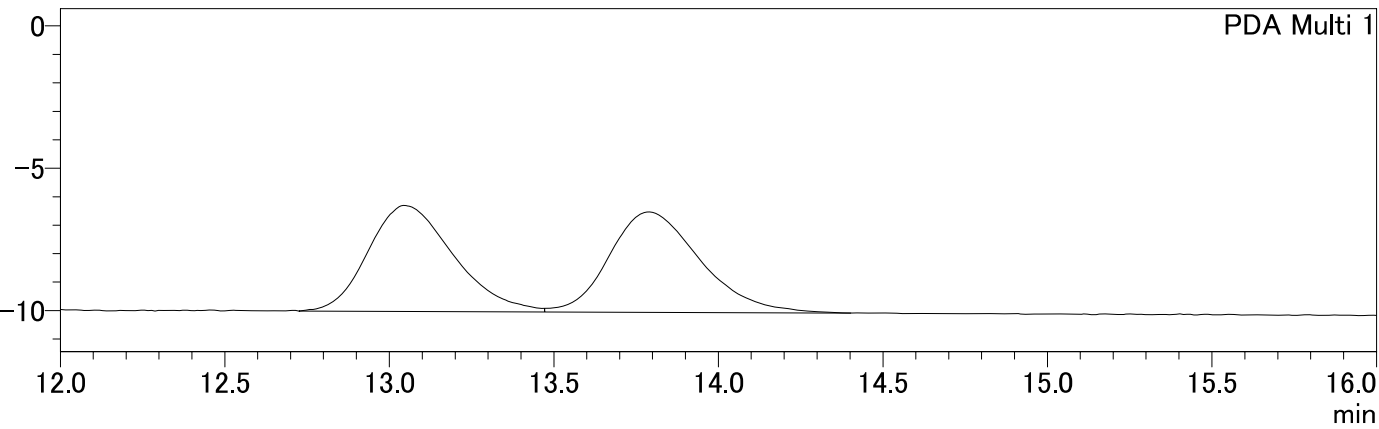

1 PDA Multi $1 / 254 \mathrm{~nm} 4 \mathrm{~nm}$

$\langle$ Peak Report>

PDA Ch1 254nm 4nm

peak table C:¥data_131217¥murakami¥HM0551-IB.lcd

peak\#

\begin{tabular}{|r|r|r|r|} 
& retention time $(\mathrm{min})$ & area & area $(\%)$ \\
\hline 1 & 13.040 & 67291 & 49.834 \\
2 & 13.784 & 67740 & 50.166
\end{tabular}

\section{$\langle$ Chromatogram〉}

chromatogram

HM0550 C:¥data_131217¥murakami¥HM0550-IB.lcd

mAU

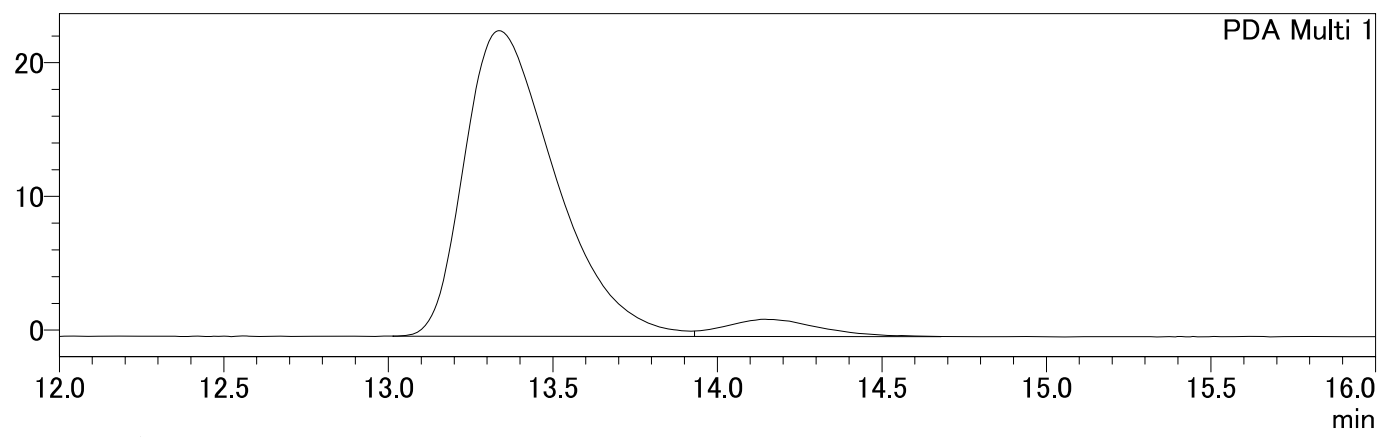

1 PDA Multi $1 / 254 \mathrm{~nm} 4 \mathrm{~nm}$

〈Peak Report>

PDA Ch1 254nm 4nm

$$
\text { peak table C:¥data_131217¥murakami¥HM0550-IB.Icd }
$$

peak\#

\begin{tabular}{r|r|} 
& retention time $(\min )$ \\
\hline 1 & 13.332 \\
2 & 14.132
\end{tabular}

13.332

area

440616

$\%)$

94.445 


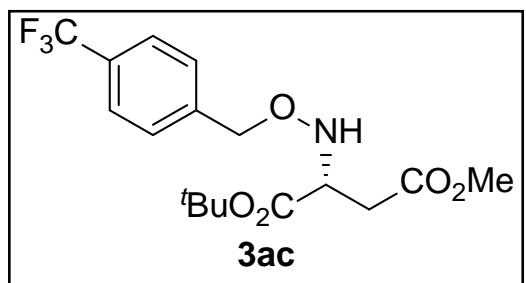

〈Chromatogram〉

chromatogram

HM0150-B C:¥data_131217¥murakami¥HM0150-B-IA001.Icd

$\mathrm{mAU}$

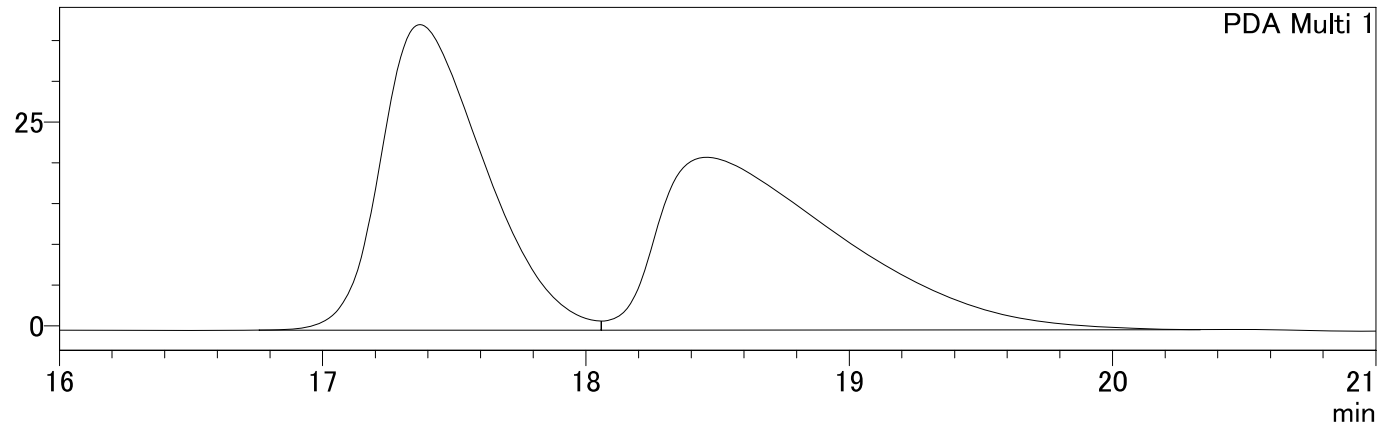

$\langle$ Peak Report $\rangle$

PDA Ch1 254nm 4nm

peak table C:¥data_131217¥murakami¥HM0150-B-IA001.lcd

peak\#

retention time $(\min )$

18.456

area

1032118

area $(\%)$

$1029236 \quad 49.930$

〈Chromatogram〉

chromatogram

HM0151-B C:¥data_131217¥murakami¥HM0151-B-IB001.Icd

mAU

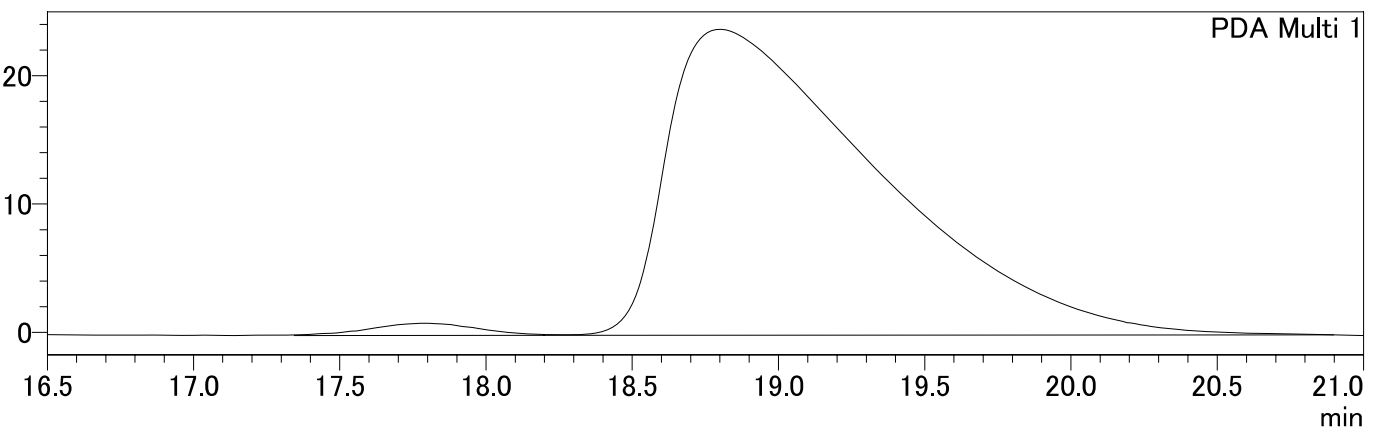

1 PDA Multi $1 / 254 \mathrm{~nm} 4 \mathrm{~nm}$

〈Peak Report〉

PDA Ch1 254nm 4nm

peak table C:¥data_131217¥murakami¥HM0151-B-IB001.Icd

\begin{tabular}{|r|r|r|r|}
\hline peak\# & retention time $(\min )$ & area & area $(\%)$ \\
\hline 1 & 17.799 & 24343 & 1.981 \\
2 & 18.800 & 1204526 & 98.019 \\
\hline
\end{tabular}




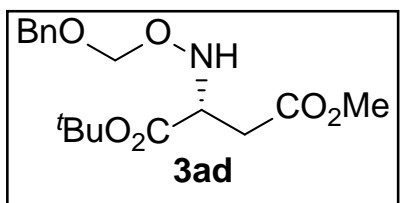

$\langle$ Chromatogram〉

chromatogram

mAU HM0135-B C:¥data_131217¥murakami¥HM0135-B-IB001.lcd

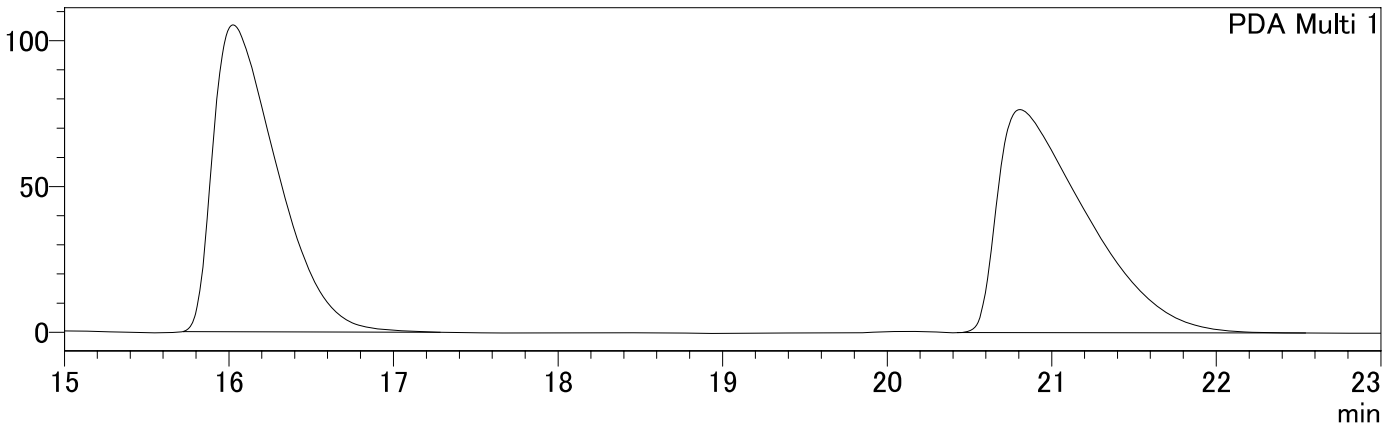

1 PDA Multi $1 / 220 \mathrm{~nm} 4 \mathrm{~nm}$ $\langle$ Peak Report>

PDA Ch1 220nm 4nm peak table C:¥data_131217¥murakami¥HM0135-B-IB001.lcd

\begin{tabular}{|r|r|r|r|}
\hline peak\# & retention time $(\min )$ & area & area $(\%)$ \\
\hline 1 & 16.019 & 2851908 & 49.822 \\
2 & 20.800 & 2872271 & 50.178 \\
\hline
\end{tabular}

〈Chromatogram〉

chromatogram

HM-136-B C:¥data_131217¥murakami¥HM0136-B-IB001.lcd

$\mathrm{mAl}$

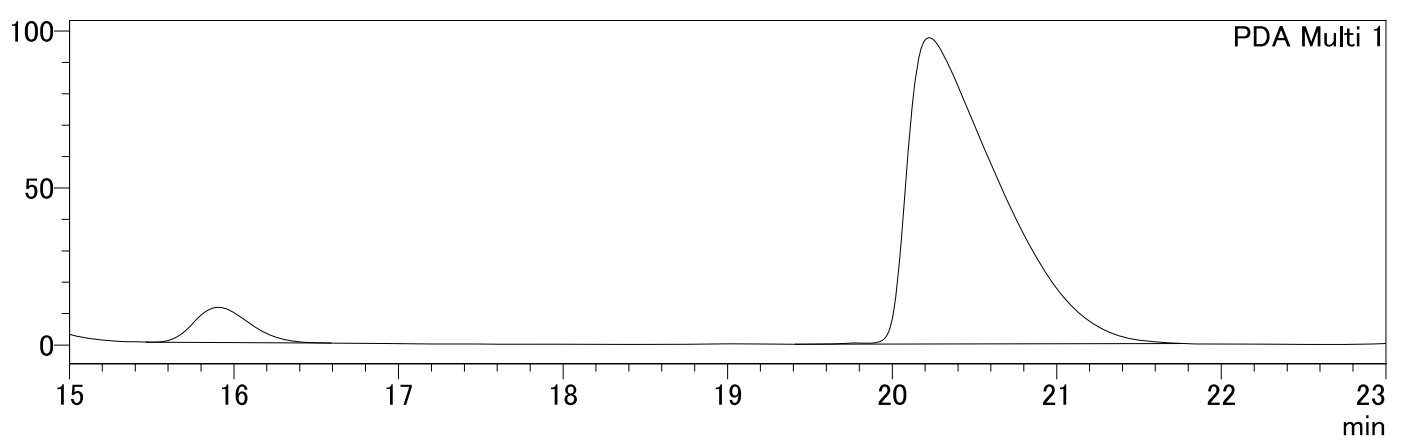

1 PDA Multi $1 / 220 \mathrm{~nm} 4 \mathrm{~nm}$

〈Peak Report>

PDA Ch1 220nm 4nm

peak table C:¥data_131217¥murakami¥HM0136-B-IB001.lcd

peak\#

retention time $(\mathrm{min})$

area $\quad$ area (\%)

1
2

20.219

3727434 


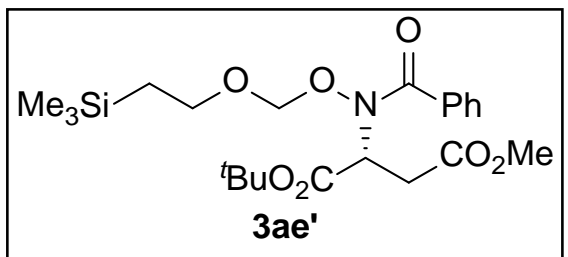

\section{$\langle$ Chromatogram〉}

chromatogram

HM0501 C:¥data_131217¥murakami¥HM0501-IB.lcd

mAU

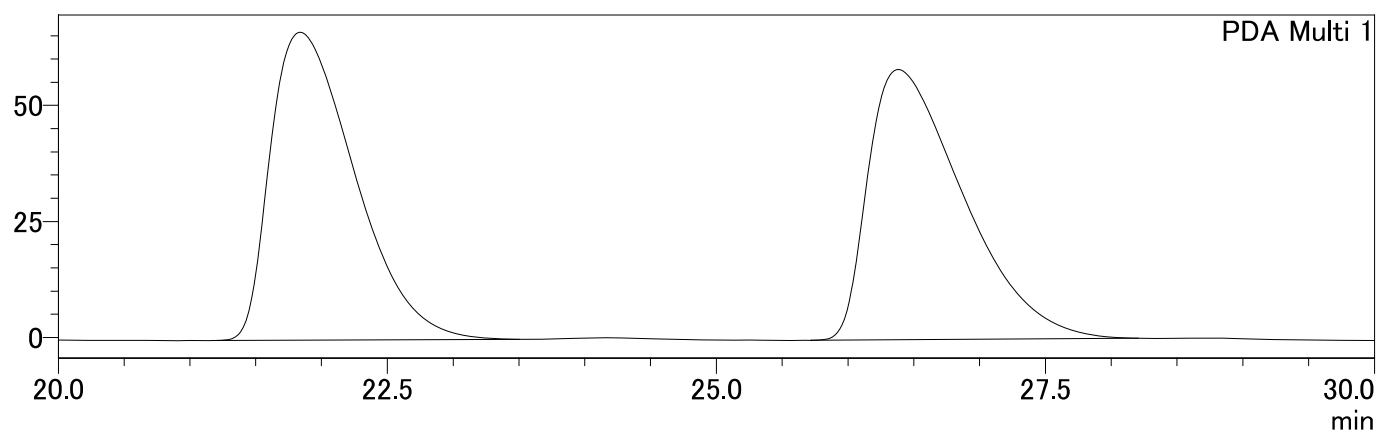

1 PDA Multi 1 / 254nm 4nm

〈Peak Report>

PDA Ch1 254nm 4nm

peak table C:¥data 131217¥murakami¥HM0501-IB.Icd

peak\#

retention time $(\mathrm{min})$

21.830

26.375

area

2954870

area (\%)

\begin{tabular}{|r|r|}
2954870 & 50.255 \\
\hline
\end{tabular}

\section{$\langle$ Chromatogram〉}

chromatogram

HM0511 C:¥data_131217¥murakami¥HM0511-IB001.lcd

$\mathrm{mAU}$

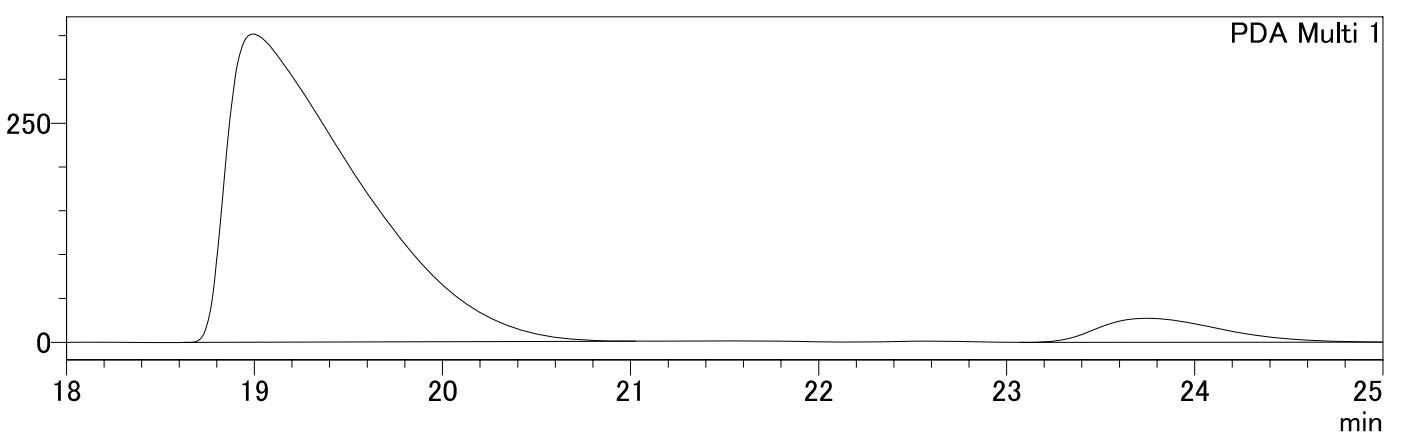

1 PDA Multi $1 / 254 \mathrm{~nm} 4 \mathrm{~nm}$

〈Peak Report>

peak table C:¥data_131217¥murakami¥HM0511-IB001.lcd

PDA Ch1 254nm 4nm

peak\#

\begin{tabular}{r|r|rr|} 
& retention time $(\mathrm{min})$ & area & area $(\%)$ \\
\hline 1 & 18.988 & 16853273 & 93.145 \\
\hline 2 & 23.742 & 1240399 & 6.855
\end{tabular}




$$
\mathrm{Me}_{3} \mathrm{Si} \underset{\substack{\mathrm{BnO}_{2} \mathrm{C}}}{\mathrm{O}} \mathrm{CO}_{2}^{\mathrm{O} M e}
$$

\section{$\langle$ Chromatogram〉}

chromatogram

HM-155-B C:¥data_131217¥murakami¥HM0155-B-IB001.lcd

$\mathrm{mAU}$

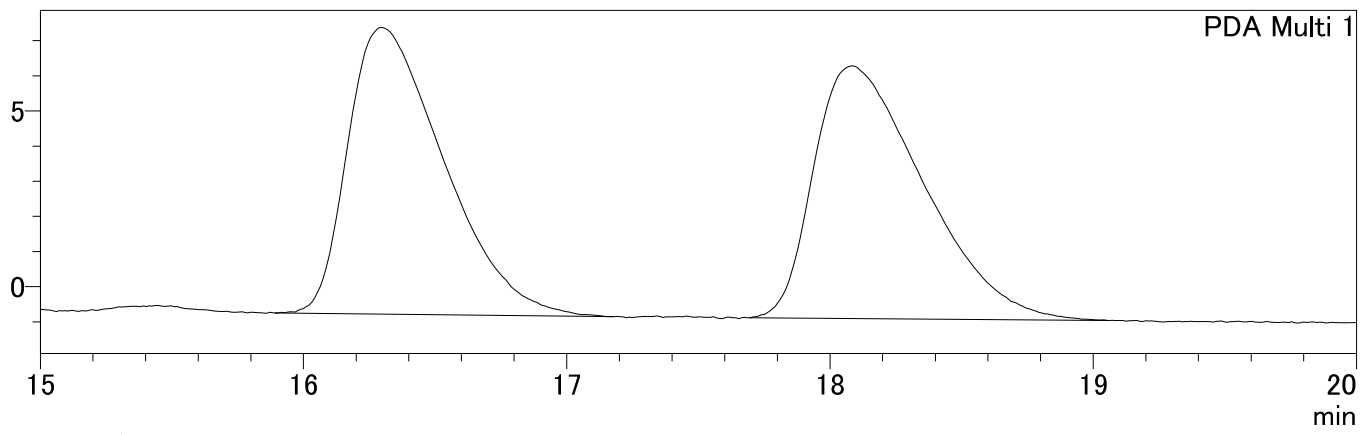

1 PDA Multi $1 / 254 \mathrm{~nm} 4 \mathrm{~nm}$

〈Peak Report>

PDA Ch1 254nm 4nm

peak table C:¥data_131217¥murakami¥HM0155-B-IB001.lcd

\begin{tabular}{|r|r|r|r|}
\hline peak\# & retention time $(\min )$ & area & area $(\%)$ \\
\hline 1 & 16.289 & 206655 & 49.880 \\
2 & 18.079 & 207647 & 50.120 \\
\hline
\end{tabular}

$\langle$ Chromatogram〉

chromatogram

HM-156-B C:¥data_131217¥murakami¥HM0156-B-IB001.lcd

$\mathrm{mAU}$

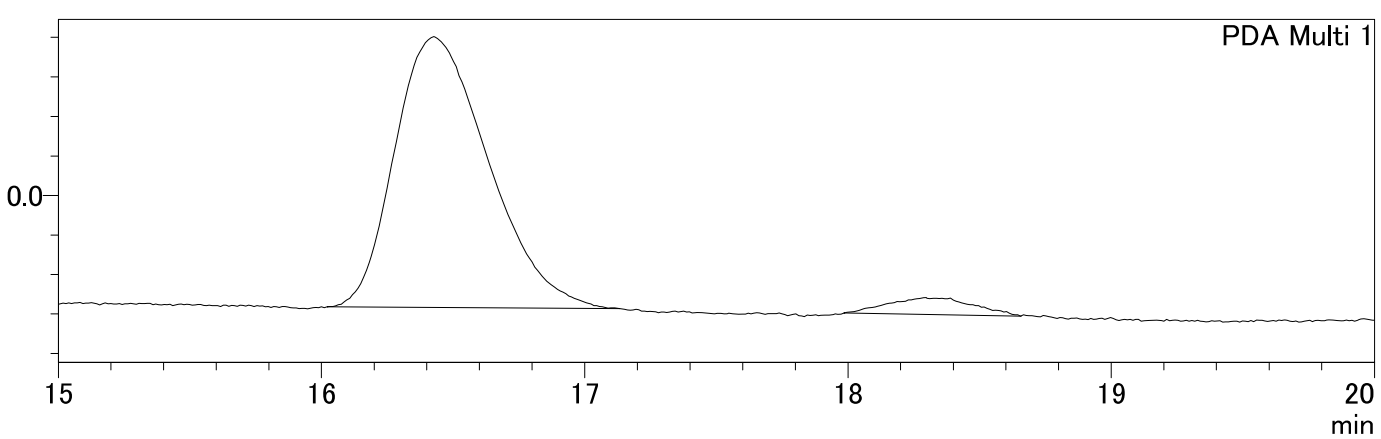

1 PDA Multi $1 / 254 \mathrm{~nm} 4 \mathrm{~nm}$

〈Peak Report>

PDA Ch1 254nm 4nm

peak table C:¥data_131217¥murakami¥HM0156-B-IB001.Icd

peak\#

retention time (min)

16.421

area

84529

area (\%)

94.632

$4795 \quad 5.368$ 


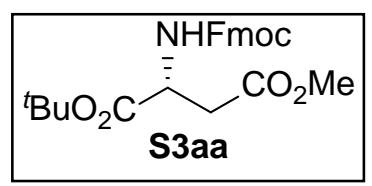

$\langle$ Chromatogram〉

chromatogram

HM0033-C C:¥data_131217¥murakami¥HM0033-C-IA.lcd

$\mathrm{mAU}$

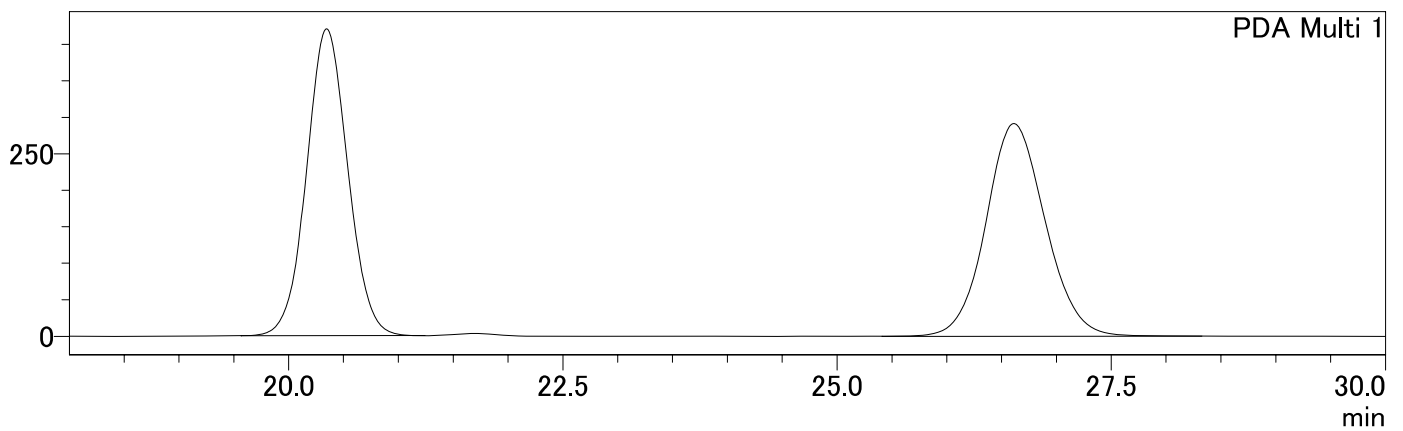

1 PDA Multi $1 / 254 \mathrm{~nm} 4 \mathrm{~nm}$

〈Peak Report>

peak table C:¥data_131217¥murakami¥HM0033-C-IA.Icd

PDA Ch1 254nm 4nm

peak\#

retention time $(\mathrm{min})$

\begin{tabular}{l|l|r}
$\min )$ & area & \multicolumn{1}{|c}{ area $(\%)$} \\
\hline 20.340 & 11004240 & 50.134 \\
26.606 & 10945554 & 49.866
\end{tabular}

〈Chromatogram〉

chromatogram

mAU

HM0045-D C:¥data_131217¥murakami¥HM0045-D-IA.Icd

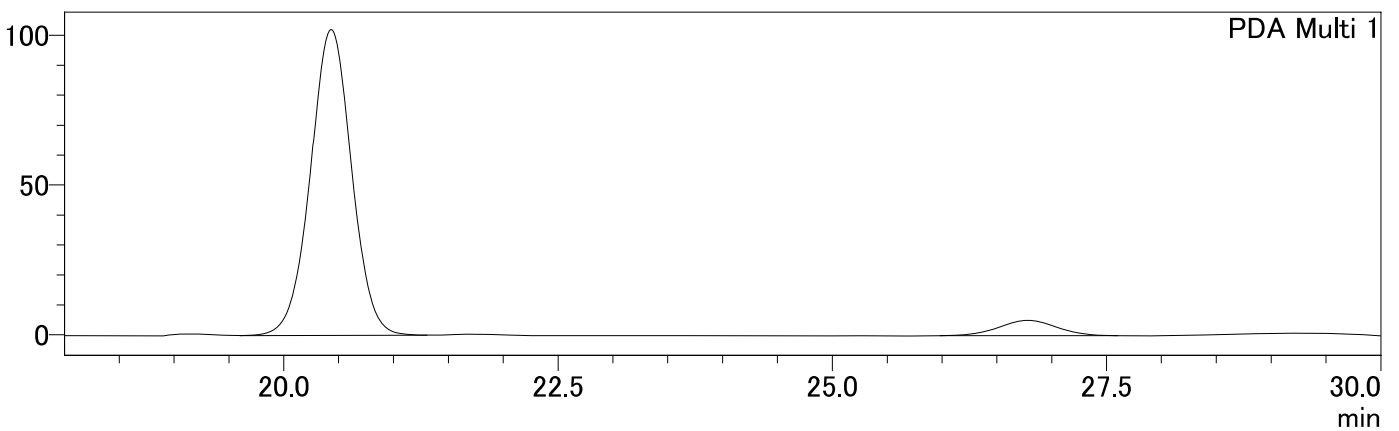

1 PDA Multi $1 / 254 \mathrm{~nm} 4 \mathrm{~nm}$

〈Peak Report>

PDA Ch1 254nm 4nm

peak table C:¥data_131217¥murakami¥HM0045-D-IA.Icd

\begin{tabular}{|r|r|r|r|}
\hline peak\# & retention time (min) & area & area (\%) \\
\hline 1 & 20.425 & 2667915 & 93.426 \\
2 & 26.772 & 187744 & 6.574 \\
\hline
\end{tabular}


<smiles>COCC(NC(=O)CCc1ccccc1)C(=O)OCc1ccccc1</smiles>

$\langle$ Chromatogram〉

chromatogram

HM-156-B C:¥data_131217¥murakami¥HM0156-B-IB001.lcd

mAU

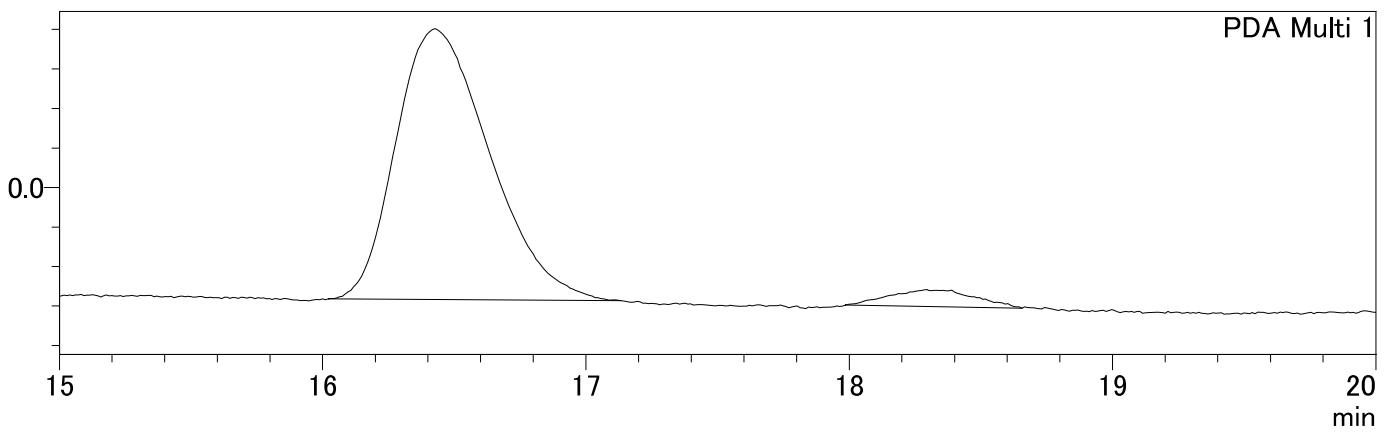

1 PDA Multi $1 / 254 \mathrm{~nm} 4 \mathrm{~nm}$

〈Peak Report>

peak table C:¥data_131217¥murakami¥HM0156-B-IB001.lcd

PDA Ch1 254nm 4nm

\begin{tabular}{|r|r|rr|}
\hline \multicolumn{1}{|c|}{ peak\# } & retention time $(\mathrm{min})$ & area & area (\%) \\
\hline 1 & 16.421 & 84529 & 94.632 \\
\hline 2 & 18.287 & 4795 & 5.368
\end{tabular}

$\langle$ Chromatogram〉

chromatogram

HM-278 C:¥data_131217¥murakami¥HM0278-IB006.lcd

mAU

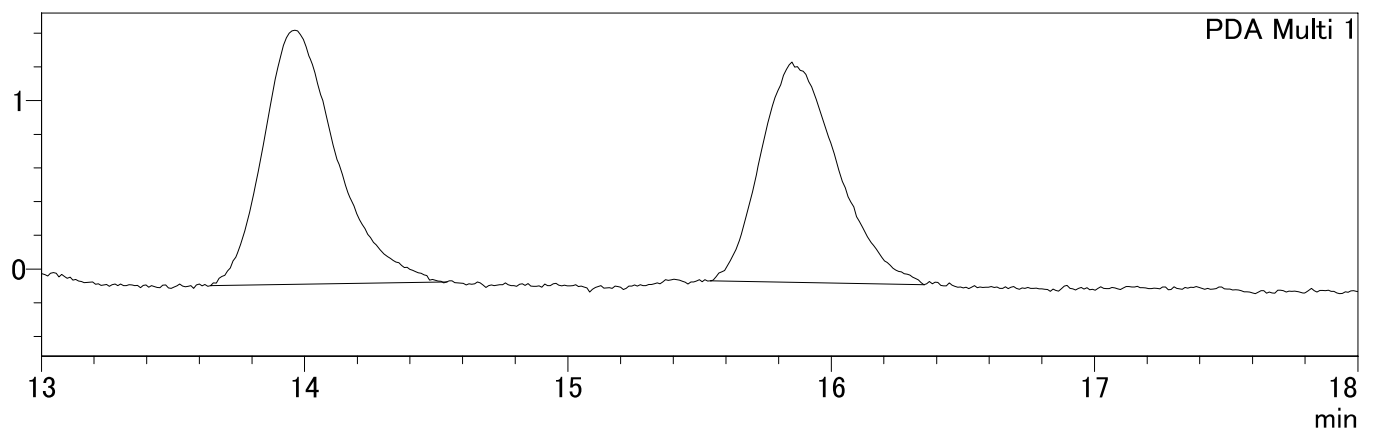

1 PDA Multi $1 / 254 \mathrm{~nm} 4 \mathrm{~nm}$

$\langle$ Peak Report>

PDA Ch1 254nm 4nm

peak table C:¥data_131217¥murakami¥HM0278-IB006.Icd

\begin{tabular}{|r|r|r|r|}
\hline \multicolumn{1}{|c|}{ peak\# } & retention time $(\mathrm{min})$ & area & area (\%) \\
\hline 1 & 13.958 & 29089 & 52.465 \\
2 & 15.844 & 26356 & 47.535 \\
\hline
\end{tabular}

